University of Louisville

ThinkIR: The University of Louisville's Institutional Repository

Electronic Theses and Dissertations

8-2020

\title{
A structural study of correlated materials: incipient mott insulators and low-dimensional systesm.
}

\author{
Alaa Alfailakawi \\ University of Louisville
}

Follow this and additional works at: https://ir.library.louisville.edu/etd

Part of the Condensed Matter Physics Commons

\section{Recommended Citation}

Alfailakawi, Alaa, "A structural study of correlated materials: incipient mott insulators and lowdimensional systesm." (2020). Electronic Theses and Dissertations. Paper 3509.

https://doi.org/10.18297/etd/3509

This Doctoral Dissertation is brought to you for free and open access by ThinkIR: The University of Louisville's Institutional Repository. It has been accepted for inclusion in Electronic Theses and Dissertations by an authorized administrator of ThinkIR: The University of Louisville's Institutional Repository. This title appears here courtesy of the author, who has retained all other copyrights. For more information, please contact thinkir@louisville.edu. 


\title{
A STRUCTURAL STUDY OF CORRELATED MATERIALS: INCIPIENT MOTT INSULATORS AND LOW-DIMENSIONAL SYSTEMS
}

\author{
By \\ Alaa Alfailakawi \\ M.Sc. in Physics, 2015 \\ A Dissertation \\ Submitted to the Faculty of the \\ College of Arts and Sciences of the University of Louisville \\ in Partial Fulfillment of the Requirements \\ for the Degree of \\ Doctor of Philosophy in Physics \\ Department of Physics and Astronomy \\ University of Louisville \\ Louisville, Kentucky
}

August 2020 
Copyright 2020 by Alaa Alfailakawi

All rights reserved 



\title{
A STRUCTURAL STUDY OF CORRELATED MATERIALS: INCIPIENT MOTT INSULATORS AND LOW-DIMENSIONAL SYSTEMS
}

\author{
By
}

Alaa Alfailakawi

M.Sc. in Physics, 2015

Dissertation approved on

May 19, 2020

by the following dissertation Committee:

\begin{tabular}{c}
\hline $\begin{array}{c}\text { Dissertation Director } \\
\text { Dr. Serban Smadici }\end{array}$ \\
\hline Dr. Byron Freelon \\
\hline Dr. Gamini Sumanasekera \\
\hline Dr. Ming Yu \\
\hline
\end{tabular}

Dr. Farshid Ramezanipour 


\section{DEDICATION}

To My family.

My parents Mahmoud and Aisha.

My sisters Mryam. Esraa and Asmaa and brother Abdualrahman.

My kids Khaled, Ahmad, Sarah, Shaikha, Moothy, Saleh and Dalal.

My husband Sabah for his continuous love and support. 


\section{ACKNOWLEDGMENTS}

I would like to express my sincere gratitude to Kuwait University financial support throughout my PhD studies. I sincerely appreciate the continues support from the Physics department, Kuwait University. I would like to thank my colleagues Bhupendra Karki, Manthila Rajapakse and Safiyah Alharbi who kindly provided lots of help. Dr. Ben Frandsen for all the help and thoughtful comments he provided during our PDF analysis Special thanks to all the national labs and beam scientists I worked with during my research. Special thanks to Dr. Ovidu Garlea, Dr. Doug Abernathy, Michelle Everett, Dr. Joerg Neuefeind, Dr. Christina Hoffmann, Dr. Simion Kimber, Melanie Kirkham, from Oak Ridge National Laboratories (ORNL). I would like 11-BM mail-in program and beamline scientists at Advanced Photon Source (APS), Argonne national laboratories. From the National Institute of Standards and Technology (NIST), I express my thanks to Dr. William Ratcliff and Dr. Colin Heikes. Also, I would like to thank Dr. Farshid Ramezanipour research group and Dr. Mark from the chemistry department at the University of Louisville for helping with lab-based x-ray. I would like to express my appreciation to Professor Minghu Fang research group, Beijing National Laboratory for Condensed Matter Physics and Zhejiang University, for growing Mott insulators and performing some transport measurement. I wish to thank all the people whose assistance was a milestone in the completion of this project Dr. Byron Freelon for his unlimited support, motivation, and thoughts. My special thank for all other committee members Dr. Serban Smadici, Dr. Gamini Sumanasekera, Dr. Ming Yu, and Dr. Farshid Ramezanipour. 


\title{
ABSTRACT \\ A STRUCTURAL STUDY OF CORRELATED MATERIALS: INCIPIENT MOTT INSULATORS AND LOW-DIMENSIONAL SYSTEMS
}

\author{
Alaa Mahmoud Alfailakawi
}

May 19, 2020

Current theories of high-temperature superconductivity suggest that electrons must organize into Cooper pairs in order for a material to exhibit a superconducting phase. Electrons in insulators experience significant repulsive interactions that tend to keep electrons localized at atomic positions. In contrast, electrons in metals are delocalized, interact weakly, and are free to conduct electricity. Therefore, the formation of Cooper pairs should have different mechanisms for metals compared to insulators. This contrast raises the debate about the origin of high-temperature superconductivity in iron-based material, whether it depends on the strong or weak coupling. Many iron-based materials are metallic in the normal phase; however, before entering the superconducting phase, iron-based superconductors are believed to harbor insulating characteristics in close proximity to a Mott insulator. Furthermore, because superconductivity in iron-based materials occurs the border of correlation-induced electronic order, it is crucial to understand the nature of the ordered states. The newly reported iron oxychalcogenide $\mathrm{Ca}_{2} \mathrm{O}_{2} \mathrm{Fe}_{2.6} \mathrm{OS}_{2}$ is an antiferromagnetic (AFM) insulator at room temperature. Oxychalcogenides are structurally similar to the ironbased superconductors and it is possible to tune the Fe-Fe ion distance to drive the material from an insulating to a metallic phase. It is unexpected that a decrease in 
the Fe-Fe ion distance for $\mathrm{Ca}_{2} \mathrm{O}_{2} \mathrm{Fe}_{2.6} \mathrm{OS}_{2}$ results in enhanced insulating properties instead of making the material more metallic. This violates the predictions of the well-established electron band theory.

The first aim in this work was to examine the novel Mott insulator $\mathrm{Ca}_{2} \mathrm{O}_{2} \mathrm{Fe}_{2.6} \mathrm{OS}_{2}$, crystal structure, and the effect of selenium doping on the material. X-ray powder diffraction (XRD) and Rietveld analysis were used to study the crystal structure. Also, neutron powder diffraction was used to study the magnetic peak intensity behavior with changes in temperature. Transport measurements were performed on both samples and activation energies $\left(\mathrm{E}_{a}\right)$ was calculated as $0.0694 \mathrm{eV}$ and 0.06098 eV for $\mathrm{Ca}_{2} \mathrm{O}_{2} \mathrm{Fe}_{2.6} \mathrm{OS}_{2}$ and $\mathrm{Ca}_{2} \mathrm{O}_{2} \mathrm{Fe}_{2.6} \mathrm{OS}_{1.75} \mathrm{Se}_{0.25}$ respectively. The Rietveld fits confirmed that the material had tetragonal crystal system with space group $P_{4} / \mathrm{mmm}$ for both samples. The calculated $\beta$ showed that this Mott insulator has the two dimensional Ising model. The volume of crystal increased with decreasing temperature while the atomic site occupancy increased. Also, the doping did not affect the crystal structure, however it suppressed the magnetic behaviour in $\mathrm{Ca}_{2} \mathrm{O}_{2} \mathrm{Fe}_{2.6} \mathrm{OS}_{1.75} \mathrm{Se}_{0.25}$.

The second aim of this work was to study the structure of iron oxychalcogenides $\mathrm{La}_{2} \mathrm{O}_{2} \mathrm{Fe}_{2} \mathrm{O}(\mathrm{S}, \mathrm{Se})_{2}$, compare the short-range to the average structure, and understand the short-range behavior in this type of Mott insulators. Neutron powder diffraction (NPD) was used to study the short and average structure of $\mathrm{La}_{2} \mathrm{O}_{2} \mathrm{Fe}_{2} \mathrm{O}(\mathrm{S}, \mathrm{Se})_{2}$. The obtained NPD data were analyzed using Rietveld analysis and pair distribution function (PDF) method. We observe the presence of fluctuating nematic ordering from low temperature to room temperature. High values of the isotropic thermal parameter $\left(U_{33}\right)$ for the oxygen atom $\mathrm{O} 2$ along $c$-axis was observed. The results also suggested the presence of short-range local distortion in both $\mathrm{La}_{2} \mathrm{O}_{2} \mathrm{Fe}_{2} \mathrm{O}(\mathrm{S}, \mathrm{Se})_{2}$ materials which can be evidence of nematic behavior in the short-range of these materials.

The last part of this research investigated the transition metal dichalcogenides 
(TMDs). TMDs are receiving a large amount of attention because they have been posited as a potential successor to silicon in the future of electronics manufacturing. The fabrication of large TMD crystals is currently an active area of industrial work. Most TMDs are low dimensional materials in the sense that their structures consist of stacks of hexagonally atomic layers of transition metal atoms or chalcogens sandwiched by transition metal (TM) layers. In addition to this, TMD electronic properties are primarily contained in the 2D TM planes. The last aim of this research was to study the transition metal dichalcogenide $\mathrm{MoTe}_{2}$ in two of its three stable phases $\left(2 \mathrm{H}-\mathrm{MoTe}_{2}\right.$ and $\left.1 T^{\prime}-\mathrm{MoTe}_{2}\right)$. Also, we studied the $\mathrm{T}_{d}$ phase, which results from the phase transition of $1 T^{\prime}-\mathrm{MoTe}_{2}$ at low temperatures.

Powder diffraction techniques were implemented to study the detail of the crystal structure of bulk phases. Rietveld analysis was used to analyze the powder diffraction data of all $\mathrm{MoTe}_{2}$ phases. Electron dispersion x-ray spectroscopy (EDX) and Raman spectroscopy were performed on both samples to check the purity of the samples. The EDX and Raman spectroscopy showed that the samples were clear from impurities. The Rietveld analysis determined the crystal structure of $2 \mathrm{H}-\mathrm{MoTe}_{2}, 1 \mathrm{~T}^{\prime}-\mathrm{MoTe}_{2}$ and $\mathrm{T}_{d^{-}} \mathrm{MoTe}_{2}$ were hexagonal $P 6_{3} / m m c$, monoclinic $P 2_{1} / m$, and orthorhombic $P m n 2_{1}$ respectively. Our results showed that temperature affected the atomic site occupancy in the studied $\mathrm{MoTe}_{2}$ phases which might be related to the stacking faults in $\mathrm{MoTe}_{2}$ layers. 


\section{TABLE OF CONTENTS}

ACKNOWLEDGMENTS ............................ iv

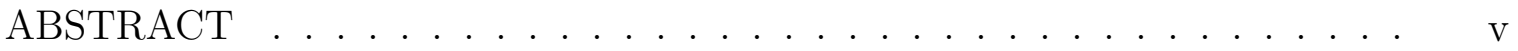

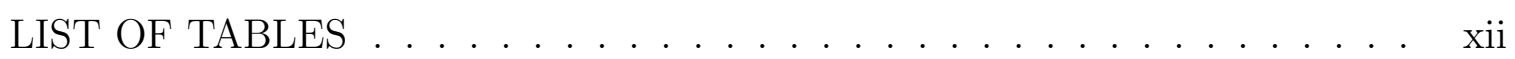

LIST OF FIGURES ...................... xiv

1 INTRODUCTION TO STRONGLY CORRELATED ELECTRONIC SYS-

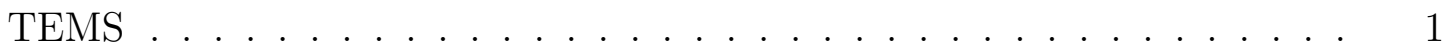

1.1 Mott Insulators . . . . . . . . . . . . . . . . . . . . 6

1.2 Iron-based Superconductors . . . . . . . . . . . . . . . . . . . . . 7

1.3 Mott insulators in this study . . . . . . . . . . . . . . . . . 9

2 LOW-DIMENSIONAL SYSTEMS . . . . . . . . . . . . . . 10

2.1 Weyl Semi Metals . . . . . . . . . . . . . . . . . . . . 11

2.2 Transition Metal Dichalcogenide . . . . . . . . . . . . . 13

2.3 Low-dimensional materials in this study . . . . . . . . . . . . . 15

3 SCATTERING THEORY . . . . . . . . . . . . . . . 16

3.1 Introduction to Scattering . . . . . . . . . . . . 16

3.2 X-Ray Scattering . . . . . . . . . . . . . . . . . . 18

3.2.1 X-Ray Production ................. 21

3.2.2 Synchrotron Beamline Used in This Research . . . . . . . . . 22 
3.2.2.1 Advanced Photon Source (APS) 11-BM . . . . . . 22

3.3 Neutron Scattering . . . . . . . . . . . . . . . 25

3.3.1 Nuclear Neutron Scattering . . . . . . . . . . . . . 27

3.3.2 Magnetic Neutron Scattering . . . . . . . . . . . 27

3.3.3 Neutron Beam Production . . . . . . . . . . . . . . . 28

3.3.4 Neutron Beamlines Used in This Research . . . . . . . . . . . 30

3.3.4.1 POWGEN .................... 30

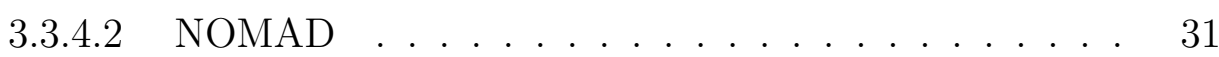

3.4 Powder Diffraction . . . . . . . . . . . . . . . . . . 32

4 ANALYSIS METHODS . . . . . . . . . . . . . . 36

4.1 Rietveld Refinement . . . . . . . . . . . . . . . . 36

4.2 Pair Distribution Function . . . . . . . . . . . . . . . . 37

4.3 Electrical Resistivity . . . . . . . . . . . . . . . 41

4.3.1 Variable-Range Hopping . . . . . . . . . . . . . . . . 41

4.4 Magnetic Properties . . . . . . . . . . . . . . . . 43

5 STRUCTURAL STUDY OF CALCIUM IRON OXYCHALCOGINIDES . 46

5.1 Introduction . . . . . . . . . . . . . . . 46

5.2 Experimental Methods ................... 51

5.2 .1 Synthesis ......................... 51

5.2.2 Diffraction Experiments . . . . . . . . . . . . . 52

$5.2 .2 .1 \quad$ X-ray diffraction . . . . . . . . . . . 52

5.2 .2 .2 Neutron diffraction . . . . . . . . . . . . 53

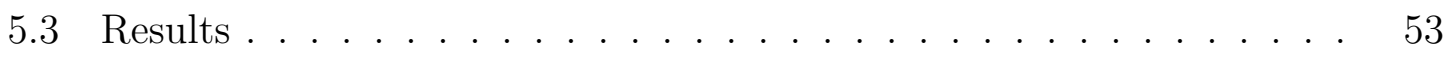

5.3.1 Electrical Resistivity Measurements . . . . . . . . . . 53

5.3.2 Magnetic Susceptibility Measurements . . . . . . . . . . 57

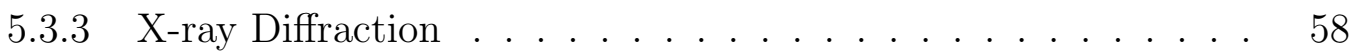


5.3.4 Neutron Powder Diffraction and Order Parameter . . . . . . . 66

5.4 Discussion . . . . . . . . . . . . . . . . . . 68

5.4.1 Electrical Resistivity Measurements . . . . . . . . . . . 68

5.4.2 Magnetic Susceptibility Measurements . . . . . . . . . . . 70

5.4 .3 X-ray Diffraction .................. 70

5.4.4 Neutron Powder Diffraction and Magnetic Order Parameter . 72

5.5 Conclusion . . . . . . . . . . . . . . . . . 73

5.6 Limitations and Future Work . . . . . . . . . . . . . . . . . . . . 74

6 LOCAL STRUCTURE OF LANTHANUM IRON OXYCHALCOGINIDES 75

6.1 Introduction . . . . . . . . . . . . . . . . . . 75

6.2 Experimental Methods . . . . . . . . . . . . . . . . . 78

6.2.1 Synthesis . . . . . . . . . . . . . . . . 78

6.2.2 Neutron Powder Diffraction . . . . . . . . . . . . . . 79

6.2.3 Pair Distribution Function . . . . . . . . . . . . . . . 79

6.3 Results........................ 80

6.4 Discussions ........................... 87

6.5 Conclusions ........................ . . . 90

6.6 Future Work . . . . . . . . . . . . . . . . . . . 90

7 STRUCTURAL STUDY OF MOLYBDENUM DITELLURIDE $2 \mathrm{H}-\mathrm{MoTe}_{2}$ AND

$1 T^{\prime}-\mathrm{MoTe}_{2} \ldots \ldots \ldots \ldots \ldots$. . . . . . . . . . . . . . . . . . 91

7.1 Introduction . . . . . . . . . . . . . . . . . . . . 91

7.2 Material . . . . . . . . . . . . . . . . 96

7.2 .1 Synthesis . . . . . . . . . . . . . . . 96

7.2.2 Characterization ................ 96

7.2.2.1 Raman Spectroscopy . . . . . . . . . . . . 96

7.2.2.2 Electron dispersion x-ray Spectroscopy (EDX) . . . . 97 
7.3 Experimental Methods . . . . . . . . . . . . . . . . . . . . . 99

7.3.1 X-ray Diffraction . . . . . . . . . . . . . . . . 99

7.3.2 Neutron diffraction . . . . . . . . . . . . . . . . . . . . 99

7.4 Results . . . . . . . . . . . . . . . . . . . 100

7.4.1 2H-MoTe$e_{2}$ Powder Diffraction . . . . . . . . . . . 100

7.4.1.1 Synchrotron X-rays . . . . . . . . . . . . . . . 100

7.4.1.2 Neutron Powder Diffraction . . . . . . . . . . . 103

7.4.2 $1 T^{\prime}-\mathrm{MoTe}_{2}$ Powder Diffraction . . . . . . . . . . . . 105

7.4.2.1 Synchrotron X-rays . . . . . . . . . . . . . . 105

7.4.2.2 Neutron Powder Diffraction . . . . . . . . . . . 109

7.5 Discussion . . . . . . . . . . . . . . . . . . . . . . 113

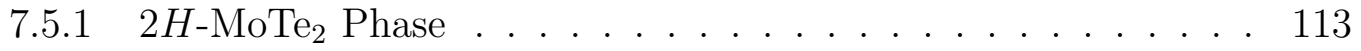

$7.5 .21 T^{\prime}-\mathrm{MoTe}_{2}$ Phase . . . . . . . . . . . . . . . . 114

7.6 Conclusion . . . . . . . . . . . . . . . . . . . . . . . . . . . 117

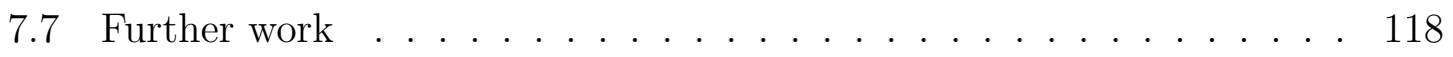

8 CONCLUSIONS . . . . . . . . . . . . . . . . . . . . . . 119

REFERENCES . . . . . . . . . . . . . . . . . . . . 122

APPENDIX A: COMMONLY USED ACRONYMS . . . . . . . . . . . 135

CURRICULUM VITA . . . . . . . . . . . . . . . . . . 136 


\section{LIST OF TABLES}

1 Activation energy calculation from the Arrhenius plots in Figure 28 using the fit function $f(x)=a_{1}+a_{2} x \ldots \ldots \ldots$. . . . . . . . . . 54

2 VRH model activation energy calculation using a fit function $f(x)=a_{1}+$ $a_{2} x$ (Figure 29) . . . . . . . . . . . . . . . . 56

3 Rietveld Refinement results for $\mathrm{Ca}_{2} \mathrm{O}_{2} \mathrm{Fe}_{2.6} \mathrm{OS}_{2}$ from room temperature lab-based XRD. Space Group P4/mmm $a=3.94485(5) \AA, c=8.7481(1)$ Åunit cell volume $=136.138(3) \AA^{3}$. . . . . . . . . . . . . . . . . . . 60

4 Rietveld Refinement results for $\mathrm{Ca}_{2} \mathrm{O}_{2} \mathrm{Fe}_{2.6} \mathrm{OS}_{1.75} \mathrm{Se}_{0.25}$ from room temperature lab-based XRD. Space Group P4/mmm $a=3.95089(6) \AA, c=$ $8.8094(2)$ Aunit cell volume $=137.510(4) \AA^{3}$. . . . . . . . . . . . . . 61

5 Proposed model for $\mathrm{Ca}_{2} \mathrm{O}_{2} \mathrm{Fe}_{2.6} \mathrm{OS}_{2}$ from 11-BM XRD at 295K. Space Group P4/mmm $a=3.94552(1) \AA, c=8.75195(2)$ Aunit cell volume $=$

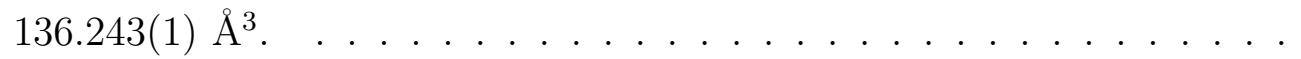

6 Proposed model for $\mathrm{Ca}_{2} \mathrm{O}_{2} \mathrm{Fe}_{2.6} \mathrm{OS}_{2}$ from 11-BM XRD at 90K. Space Group P4/mmm $a=3.94091(1) \AA, c=8.70806(3) \AA$ Anit cell volume $=135.243(1)$

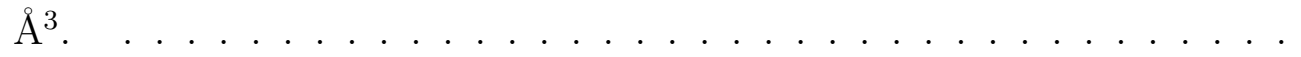

7 Proposed model for $\mathrm{Ca}_{2} \mathrm{O}_{2} \mathrm{Fe}_{2.6} \mathrm{OS}_{1.75} \mathrm{Se}_{0.25}$ from 11-BM XRD at $295 \mathrm{~K}$. Space Group P4/mmm $a=3.952598(1) \AA, c=8.813372(2) \AA$, unit cell volume $=137.692(1) \AA^{3} \ldots \ldots \ldots \ldots \ldots$

8 Proposed model for $\mathrm{Ca}_{2} \mathrm{O}_{2} \mathrm{Fe}_{2.6} \mathrm{OS}_{1.75} \mathrm{Se}_{0.25}$ from 11-BM XRD at 90K. Space Group P4/mmm $a=3.94752(1) \AA, c=8.77023(2) \AA$, unit cell volume $=136.666(1) \AA^{3} \ldots \ldots \ldots \ldots \ldots$ 
9 Activation energy values and critical exponent for the magnetic order parameter for some iron oxychalcogenides. The values for our $\mathrm{E}_{a}$ values are the average values for all temperature ranges from Table $1 . . . . . .$.

10 Rietveld Refinement results for $\mathrm{La}_{2} \mathrm{O}_{2} \mathrm{Fe}_{2} \mathrm{OSe}_{2}$ from room temperature neutron powder diffraction. Space Group $I 4 / m m m a=4.08875(2) \AA, c$ $=18.6081(1) \AA$ and unit cell volume $=311.088(4) \AA^{3} \ldots \ldots$. . . . .

11 Rietveld Refinement results for from neutron powder diffraction at $300 \mathrm{~K}$. Space Group $I 4 / m m m a=4.04388(3) \AA, c=17.89412(19) \AA$ and unit

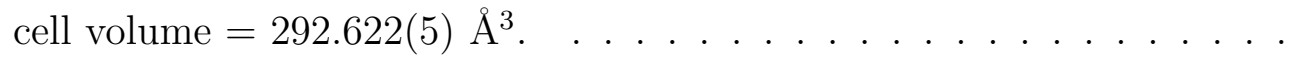

12 Schematics of the structural polytypes of $\mathrm{MoTe}_{2}$. . . . . . . . . 92

13 Proposed model for $2 \mathrm{H}-\mathrm{MoTe}_{2}$ based on 11-BM XRD at 295K. Space Group $P 6_{3} / m_{m c} a=3.51981(3) \AA, c=13.96812(7)$ A unit cell volume $=$ $149.867(2) \AA^{3}$. . . . . . . . . . . . . . . . 101

14 Proposed model for $2 \mathrm{H}-\mathrm{MoTe}_{2}$ from 11-BM XRD at 100K. Space Group $P 6_{3} / m m c a=3.51473(2) \AA, c=13.94093(7)$ A unit cell volume $=149.144(2)$ $\AA^{3}$.

15 Proposed model for $1 T^{\prime}-\mathrm{MoTe}_{2}$ from 11-BM XRD at 295K. Space Group $P 2_{1} / m a=6.32049(19) \AA, b=3.47182(14) \AA, c=13.83659(27) \AA, \beta=$ $92.5489^{\circ}(19)$, unit cell volume $=303.325(13) \AA^{3}$. . . . . . . . . . . 108

16 Proposed model for $T_{d}-\mathrm{MoTe}_{2}$ from 11-BM XRD at 100K. Space Group $P m n 2_{1} a=3.46675(29) \AA, b=6.30310(53) \AA, c=13.85899(39) \AA, \beta=$ $90.0^{\circ}(0)$, unit cell volume $=302.837(31) \AA^{3}$. The fit quality here is low and the negative values of the $U_{i s o}$ and the large fit quality parameters listed below. 


\section{LIST OF FIGURES}

1 Crystallographic and magnetic structures of (left) The tetragonal structures known to support superconductivity in FeSCs. (On right) the iron atoms planar layer which is common to all FeSCs (iron ions shown in red and pnictogen/chalcogen anions shown in gold) from reference [1]. . . . 2

2 Classification of materials based on their band structure . . . . . . . . 3

3 Mott-insulator transition phase diagram based on the Hubbard model. The strength of Coulombic repulsion $U$ and the temperature are plotted based, on the Hubbard model. At low temperature, the system had a long-range order (red). In the orange region, the model has two distinct solutions bounded by lines $U_{C 1}$ and $U_{C 2}$, the dotted line indicates first order transition in equilibrium. The first order transition line terminates at a second order critical point. The Figure is from reference [2]. . . . . .

4 Different $\mathrm{MX}_{2}$ compounds and binary compounds of group-IV elements and group III-V. The structure $(H$ or $T)$ is the ground state. The resulting structures $(T$ or $H)$ can be half-metallic $(+)$, metallic $(*)$, or semiconducting $(* *)$ with direct or indirect band gap [3] . . . . . . . . . . . . . 11

5 Schematics of the topological insulator and Weyl semimetal [4]. . . . . . 13

6 Scattering process of a particle scattering from a sample in a) and b) shows the scattering vector $\vec{Q}$. This relation holds for any type of particles. In elastic scattering $\left|K_{i}\right|=\left|K_{f}\right|$, while for inelastic scattering $\left|K_{i}\right| \neq\left|K_{f}\right| . \quad$. 16

7 The figure illustrates neutron interactions with the matter which produce nuclear and magnetic scattering . . . . . . . . . . . . . . 
9 Comparison between x-ray and neutron form factors. All form factors are normalizes to unity at $\frac{\sin \theta}{\lambda}=0 . \ldots \ldots \ldots$. . . . . . . . . 20

10 Comparison between Bragg and Laue scattering. . . . . . . . . . . . . . . 21

11 APS plan view shows every beamline and the different parts of the synchrotron from https://www.aps.anl.gov/Beamlines . . . . . . . . . 23

12 11-BM sample holder consists of a mounting base, a magnetic cap, and a capillary tube. The mounting base he a unique ten-digit barcode to tracks the sample at all steps of our mail-in service. A magnetic ring secures caps on mounting bases and provides some protection for sample capillary tubes during shipment. The Kapton tubes have an inner diameter of $0.80 \mathrm{~mm}$, and an outer diameter that fits snugly inside 11-BM mounting base. On the left, the base and the tube filled with a sample is shown. On the right, the picture shows samples bases and capillary tubes, with the protective

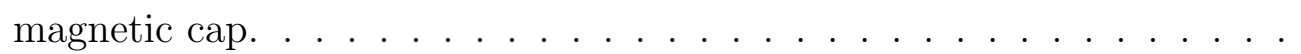

13 Length and time domains available with neutron scattering techniques compared to other investigation methods. Source https://mlz-garching.de/ englisch/neutron-research/experimental-methods/elastic-scattering.html . 26

14 Geometry of the neutron magnetic scattering. The vectors $\hat{\kappa}, \hat{\mu}$, and $\hat{P}$ are the scattering vector, the atom magnetic moment, and polarization vector respectively. $\theta$ is the angle between incident or scattered neutron and the reflective plane. $\alpha$ is the angle between the scattering vector $\hat{\kappa}$ and the magnetization vector $\hat{\mu}$. The magnetic interaction vector $\vec{q}$ lies in the plane of $\hat{\kappa}$ and $\hat{\mu}$ and is perpendicular to $\hat{\kappa}$ (adapted from $[5,6]$ ). . . 
15 Panels a and b show the Ewald construction. In panel a, the 2D section of the reciprocal lattice is shown, which represents a sphere with radius $1 / \lambda$. The incident, scattered wavevectors are $\overrightarrow{k_{0}} / 2 \pi$ and $\vec{k} / 2 \pi$. The scattering vector is $\vec{\kappa} / 2 \pi$. The diffraction condition is satisfied when the scattering vector ends at a reciprocal lattice point. Panel b shows the Ewald construction for powder diffraction were randomly oriented crystallites cause lattice points to be replaced by concentric spheres. The dashed line shows the cone of diffraction (Debye-Scherrer), which results as an intersection between the concentric sphere and the reflection sphere. Panel c shows an illustration of Debye-Scherrer concentric cones as a result of scattering from a powder sample. All illustrations are from reference [5] . . . . . . . 34

16 A powder diffraction ring pattern from silver behenate (a layered organic crystal) and the radial line plot of the scattered intensity (from https://www.physics.upenn.edu/ heiney/datasqueeze/basics.html). . . .

17 PDF method measure the inter-atomic distances between atoms in the crystal. Each peak corresponds to a distance, where neighbouring atoms are most likely found in the sample. . . . . . . . . . . . . . .

18 Metal-insulator-transition features the figure from reference [7].In Mott insulators, the metal-to-insulator (MIT) transition can be driven by different kind of external perturbations. . . . . . . . . . . . . . . 42

19 Temperature dependent magnetic susceptibility behavior in different materials adapted from $[8] \ldots \ldots \ldots \ldots$ 
20 Temperature dependant magnetic susceptibility in iron oxychalcogenides Mott insulators showing the broad maximum that characterize magnetic susceptibility in this material. Panels a and b show magnetic susceptibility in $\mathrm{La}_{2} \mathrm{O}_{2} \mathrm{Fe}_{2} \mathrm{OSe}_{2}$ and $\mathrm{La}_{2} \mathrm{O}_{2} \mathrm{Fe}_{2} \mathrm{OS}_{2}$ respectively, where a Curie tails are observed at low temperature from [9]. Panel c) shows a typical magnetic susceptibility in iron oxychalcogenides as in $\mathrm{Sr}_{2} \mathrm{~F}_{2} \mathrm{Fe}_{2} \mathrm{OS}_{2}$ and $\mathrm{Ba}_{2} \mathrm{~F}_{2} \mathrm{Fe}_{2} \mathrm{OS}_{2}$ from $[10] \ldots \ldots \ldots \ldots$

$21 \mathrm{Ca}_{2} \mathrm{O}_{2} \mathrm{Fe}_{2.6} \mathrm{OS}_{2}$ resistivity and magnetic susceptibility from reference [11].

22 Pnictide phase diagram near a magnetic quantum critical point. The blue dot denotes the quantum critical point determined by the critical tuning parameter $\mathrm{w}_{c}$. The blue line is the line of the thermally driven antiferromagnetic transition and the dashed line is a structural transition [12]. The red star represents the location of $\mathrm{Ca}_{2} \mathrm{O}_{2} \mathrm{Fe}_{2.6} \mathrm{OS}_{2}$ within the phase diagram. . . . . . . . . . . . . . . . . . .

23 The crystal structure of $\mathrm{Ca}_{2} \mathrm{O}_{2} \mathrm{Fe}_{2.6} \mathrm{OS}_{2}$ is shown in panel a). Panel b) shows the $\mathrm{Fe} 2$ and $\mathrm{O} 2$ atoms forming the square-planer in the $\mathrm{Fe}_{2} \mathrm{OS}_{2}$ layer. The Fe-Fe distance $\left(D_{F e-F e}\right)$ between any two iron atoms in this

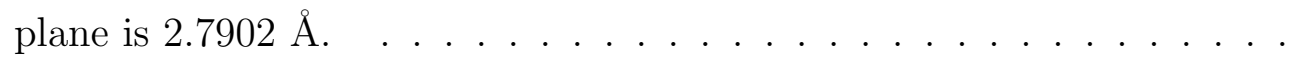

24 The octahedra in $\mathrm{Ca}_{2} \mathrm{O}_{2} \mathrm{Fe}_{2.6} \mathrm{OS}_{2}$ structure. Each octahedron consists of an $\mathrm{Fe} 2$ ion, two $\mathrm{O} 2$ ions, and four $\mathrm{S}$ ions forming $\mathrm{FeO}_{2} \mathrm{~S}_{4} \ldots \ldots .$.

25 Activation energy dependence of the Fe-Fe bond length $\left(D_{F e-F e}\right)$ iron oxychalcogenides including the materiel in this study which are shown in gray and green stars. Pink markers shows iron based superconductors. Activation energy and bond length values used in this graph were from references $[11,13,9,14,15] \ldots \ldots \ldots \ldots$

26 Powdering a sample with mortar and pestle as part of sample prepration for POWGEN experiment. . . . . . . . . . . . . . . 52 
27 Temperature dependent of resistivity $\rho(T)$ for $\mathrm{Ca}_{2} \mathrm{O}_{2} \mathrm{Fe}_{2.6} \mathrm{OS}_{2-x} \mathrm{Se}_{x} \quad(x=$

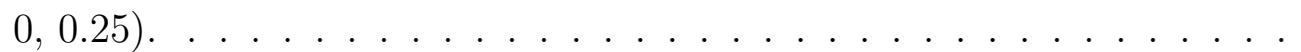

28 Resistivity measurement data above $150 \mathrm{~K}$ is used to fit the thermal activation model $\rho=\rho_{0} \exp \left(\frac{E_{a}}{K T}\right)$. Panel a) and b) show the fits for $\mathrm{Ca}_{2} \mathrm{O}_{2} \mathrm{Fe}_{2.6} \mathrm{OS}_{2}$ and $\mathrm{Ca}_{2} \mathrm{O}_{2} \mathrm{Fe}_{2.6} \mathrm{OS}_{1.75} \mathrm{Se}_{0.25}$, respectively. . . . . . . . 55

29 Temperature dependant resistivity measurements are fitted to variablerange hopping VRH model. . . . . . . . . . . . . . . . . . . . . 56

30 Temperature dependent magnetic susceptibility measurement showing antiferromagnetic behaviour for a) $\mathrm{Ca}_{2} \mathrm{O}_{2} \mathrm{Fe}_{2.6} \mathrm{OS}_{2}$, and b) $\mathrm{Ca}_{2} \mathrm{O}_{2} \mathrm{Fe}_{2.6} \mathrm{OS}_{1.75} \mathrm{Se}_{0.25}$.

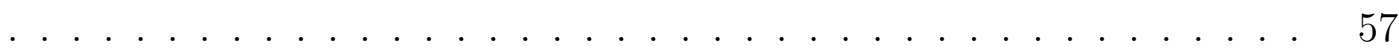

31 Rietveld Refinement profiles for the pure sample $\mathrm{Ca}_{2} \mathrm{O}_{2} \mathrm{Fe}_{2.6} \mathrm{OS}_{2}$ in the upper panel and the doped sampleCa $\mathrm{O}_{2} \mathrm{Fe}_{2.6} \mathrm{OS}_{1.75} \mathrm{Se}_{0.25}$ in the lower panel, from room temperature lab-based XRD. . . . . . . . . . . . .

32 X-ray powder diffraction from 11-BM, APS of $\mathrm{Ca}_{2} \mathrm{O}_{2} \mathrm{Fe}_{2.6} \mathrm{OS}_{2}$ at temperatures $90 \mathrm{~K}$ and $295 \mathrm{~K} \ldots \ldots \ldots$. . . . . . . . . . . . . . 60

33 X-ray powder diffraction from 11-BM, APS of $\mathrm{Ca}_{2} \mathrm{O}_{2} \mathrm{Fe}_{2.6} \mathrm{OS}_{1.75} \mathrm{Se}_{0.25}$ at temperatures $90 \mathrm{~K}$ and $295 \mathrm{~K} \ldots \ldots \ldots$. . . . . . . . . . . . 61

34 Neutron powder diffraction from POWGEN of $\mathrm{Ca}_{2} \mathrm{O}_{2} \mathrm{Fe}_{2.6} \mathrm{OS}_{2}$ at temperatures $10 \mathrm{~K}, 150 \mathrm{~K}$ and $300 \mathrm{~K}$.the insert show the evolution of the most intense magnetic peak with temperature. . . . . . . . . . . . . 66

35 Neutron powder diffraction from POWGEN of $\mathrm{Ca}_{2} \mathrm{O}_{2} \mathrm{Fe}_{2.6} \mathrm{OS}_{1.75} \mathrm{Se}_{0.25}$ at temperatures $10 \mathrm{~K}, 150 \mathrm{~K}$ and $300 \mathrm{~K} \ldots \ldots . \ldots 67$ 
36 Order parameter fitting for $\mathrm{Ca}_{2} \mathrm{O}_{2} \mathrm{Fe}_{2.6} \mathrm{OS}_{2}$ sample based on temperature dependence neutron powder diffraction calculated from the magnetic peak at T.O.F. $1.324 \times 10^{+5} \mu s\left(d_{h k l}=4.364 \AA\right)$. The order parameter describes the development of magnetic order. In $\mathrm{Ca}_{2} \mathrm{O}_{2} \mathrm{Fe}_{2.6} \mathrm{OS}_{2}$ the continuous magnetic phase transition is from the paramagnetic phase for $T>T_{N}$ to the antiferromagnetic phase when $T<T_{N}$. The magnetic transition temperature $T_{N}$ based on this fitting is $75.01 \pm 0.0194 \mathrm{~K} . \quad$. . . . . .

37 Crystal structure of lanthanum iron oxychalcogenide (left) and the octahedra (right), where the Fe atom is surrounded by four Se atoms and two

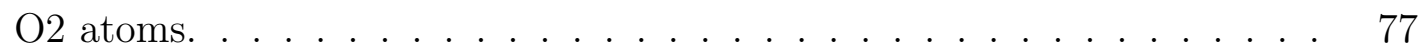

38 Panel a) shows possible Fe and the chalcogen atomic movement within the $\mathrm{La}_{2} \mathrm{O}_{2} \mathrm{Fe}_{2} \mathrm{O}(\mathrm{S}, \mathrm{Se})_{2}$ octahedra. The dashed lines in $\left.\mathrm{b}\right)$ show the $\mathrm{O} 2$ atom movement in the $\mathrm{Fe}_{2} \mathrm{O}$ plane. The solid lines show $\mathrm{Fe}$ atom movement with respect to the chalcogen atom $M[16] \ldots \ldots \ldots$

39 Nuclear structure Rietveld refinement profile of NPD of a) $M=\mathrm{S}$ and b) $M=$ Se at 300 K. . . . . . . . . . . . . . . . . . 83

40 The orthorhombicity determined from neutron PDF analysis for a) $M=$ $\mathrm{S}$ and b) $M=\mathrm{Se}$. At the short ranges over all temperatures, we observe the presence of orthorhombicity for both samples. . . . . . . . . . .

41 A comparison of phase diagram in iron pnictides superconductors and the preliminary phase diagram of $\mathrm{La}_{2} \mathrm{O}_{2} \mathrm{Fe}_{2} \mathrm{O}(\mathrm{S}, \mathrm{Se})_{2}$ Mott insulators. Panel a) after References $[17,18,19,20] \ldots \ldots \ldots$

42 Prismatic coordination of $\mathrm{MoTe}_{2}$. Panel a), b) and c) shows the coordination for $2 H-\mathrm{MoTe}_{2}, 1 T^{\prime}-\mathrm{MoTe}_{2}$, and $T_{d}-\mathrm{MoTe}_{2}$ phase. . . . . . . . . 92 
43 The structure of different phases of $\mathrm{MoTe}_{2}$. Panel a) shows top view of $2 H-\mathrm{MoTe}_{2}$, panel b) side view of $2 H-\mathrm{MoTe}_{2}$. The top view of $1 T^{\prime}-\mathrm{MoTe}_{2}$ is shown in panel c), whereas panel d) shown the side view of the same phase. The $T_{d}-\mathrm{MoTe}_{2}$ top view is shown in panel e) and the side view on panel f). 94

44 single crystal $1 T^{\prime}-\mathrm{MoTe}_{2} \ldots \ldots \ldots \ldots \ldots$. . . . . . . . . . 95

45 Left panel shows Raman Measurement on powder sample of $2 \mathrm{H}-\mathrm{MoTe}_{2}$ and $1 T^{\prime}-\mathrm{MoTe}_{2}$. Right panel shows the Raman spectra for both samples from reference $[21] \ldots \ldots \ldots \ldots \ldots \ldots \ldots$

46 Stoichiometric analysis of a) $2 \mathrm{H}-\mathrm{MoTe}_{2}$ and b) $1 T^{\prime}-\mathrm{MoTe}_{2}$ by Energydispersive x-ray spectroscopy $(\mathrm{EDX}) \ldots \ldots \ldots$

47 Panel a) presents the x-ray diffraction pattern from 11-BM at APS of $2 \mathrm{H}-\mathrm{MoTe}_{2}$ at $295 \mathrm{~K}$ and $100 \mathrm{~K}$. Panel b) shows the diffraction difference between the patterns. . . . . . . . . . . . . . . . . 102

48 Rietveld refinement profiles of x-ray diffraction data from 11-BM at APS of $2 \mathrm{H}-\mathrm{MoTe}_{2}$. a) At $\left.300 \mathrm{~K}, \mathrm{~b}\right)$ at $100 \mathrm{~K} \ldots \ldots \ldots \ldots$

49 Panel a) shows $2 \mathrm{H}-\mathrm{MoTe}_{2}$ neutron powder diffraction data from $\mathrm{C} 2$ diffractometer at Chalk river with $\lambda=1.3 \AA$ performed at temperatures 5, 200 and $320 \mathrm{~K}$. Panel b) shows magnification of the diffraction pattern highlighting the $2 \theta$ from $81.5^{\circ}$ to $97.5^{\circ} \ldots \ldots \ldots \ldots \ldots$

$502 \mathrm{H}-\mathrm{MoTe}_{2}$ neutron powder diffraction data from $\mathrm{C} 2$ diffractometer at Chalk river with $\lambda=2.37 \AA$ performed at 5, 132, 200, 208 and 320

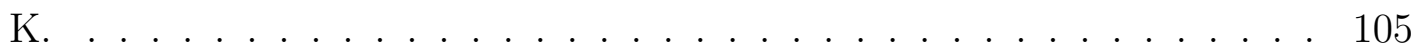

51 X-ray diffraction pattern from 11-BM at APS of $1 T^{\prime}-\mathrm{MoTe}_{2}$ at $295 \mathrm{~K}$ and $100 \mathrm{~K}$. Panel a) shows the full diffraction pattern and panel b) shows the difference between the patterns. . . . . . . . . . . . . . . 106 
52 Focused section for the comparison from the x-ray diffraction pattern of data taken at 11-BM at APS, of $1 T^{\prime}-\mathrm{MoTe}_{2}$ at $295 \mathrm{~K}$ (red) and $100 \mathrm{~K}$ (blue). The full patterns are shown in figure 51. . . . . . . . . . . . . 107

53 X-ray diffraction pattern from 11-BM at APS of $1 T^{\prime}-\mathrm{MoTe}_{2}$ At $295 \mathrm{~K}$ and $100 \mathrm{~K}$. Panel a) shows the full diffraction pattern where panels b-f shows focused portions of this pattern. . . . . . . . . . . . . . 110

54 Panel a) $1 T^{\prime}-\mathrm{MoTe}_{2}$ temperature dependence neutron powder diffraction data from C2 diffractometer at Chalk River with $\lambda=1.33 \AA$. Panel b) $1 T^{\prime}$ $\mathrm{MoTe}_{2}$ temperature dependence neutron powder diffraction data from C2 diffractometer at Chalk River with $\lambda=2.37 \AA$. . . . . . . . . . . . . 111

$551 T^{\prime}-\mathrm{MoTe}_{2}$ temperature dependence neutron powder diffraction data from C5 diffractometer at Chalk river with $\lambda=2.44 \AA$. . . . . . . . . . . . . . 112

56 Comparison between X-ray powder diffraction patterns from APS experiment and simulated patterns using GSAS-II software. Panel a) shows a full range patterns for $1 T^{\prime}-\mathrm{MoTe}_{2}$ and $T_{d}-\mathrm{MoTe}_{2}$. A focused portion of the patterns are shown in b) and c) for phases $1 T^{\prime}-\mathrm{MoTe}_{2}$ and $T_{d}-\mathrm{MoTe}_{2}$, respectively. ............................ 116 


\section{CHAPTER 1}

\section{INTRODUCTION TO STRONGLY CORRELATED ELECTRONIC SYSTEMS}

Strongly correlated electronic systems (SCES) are fascinating systems that encompass rich phenomena from the perspective of fundamental science and offer possibilities for technological applications. Discovering the relationship between the complex degrees of freedom in those SCES has been a goal for condensed matter physicists in recent years. The mechanism of superconductivity in high transition temperature (high- $\mathrm{T}_{c}$ ) superconductors (HTSC), the role of magnetic fluctuations, structural fluctuations, and even the homogeneity of the structures are topics of current interest. Furthermore, some new discoveries are groundbreaking enough to completely shift our understanding of these materials. The discovery of high- $\mathrm{T}_{c}$ superconductivity in iron-based materials, in 2008, paved the way for discovering a new superconducting family of materials $[22,23]$. Figure 1 shows crystallographic structure and magnetic structure for some of FeSCs materials.

SCES often have remarkable properties and transitions between distinct, competing phases with dramatically different electronic and magnetic orders. These SCESs phenomena are often challenging to describe in a single definition due to their complexity. It is worth noting that in SCES, the interaction between electrons in solids is of fundamental importance as it governs the behavior of the whole system. SCESs show the strong interplay between different degrees of freedom, charge, orbital, spin,

and lattice, which leads to novel material properties. These materials often have a complex phase diagram. SCESs includes much material such as conventional su- 


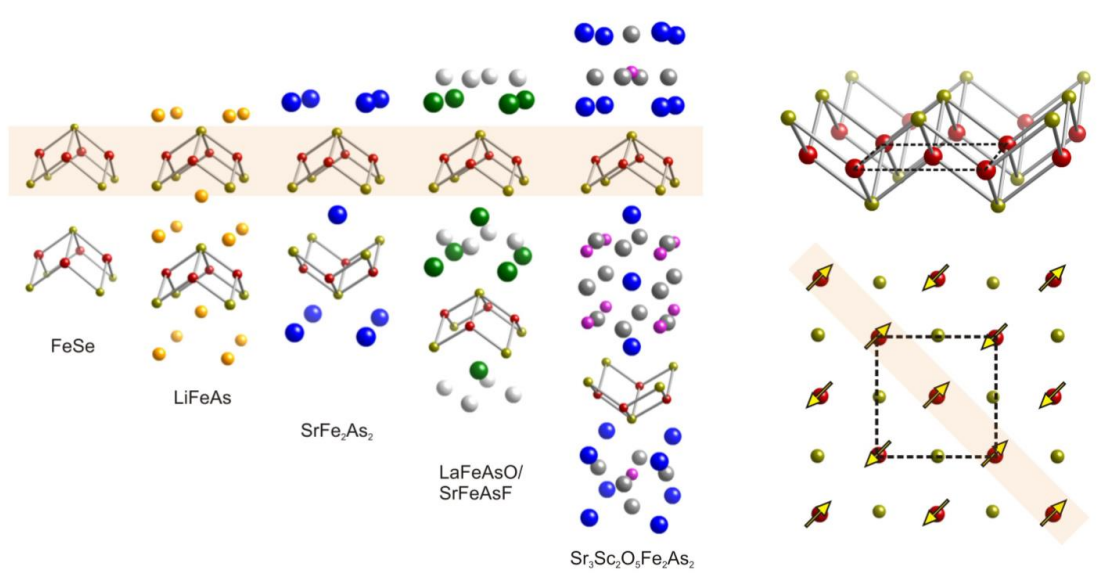

Figure 1. Crystallographic and magnetic structures of (left) The tetragonal structures known to support superconductivity in FeSCs. (On right) the iron atoms planar layer which is common to all FeSCs (iron ions shown in red and pnictogen/chalcogen anions shown in gold) from reference [1].

perconductors and High-temperature superconductors, quantum hall systems (QHS), organic conductors, spintronic materials, multiferroics, Mott insulators, spin Peierls materials, and heavy fermion materials.

Two distinctive features of iron-based materials are their complex phase interplay and a surprisingly large chemical variety. The presence of high $T_{c}$ superconductivity in doped Mott insulators such as the cuprates contradicts the conventional thought that electron repulsion is detrimental to the presence of superconductivity. Bloch theorem is used to describe conventional condensed matter materials by assuming non-interacting electrons to describe the band structure. Based on this assumption, the band structure of materials can be categorized as metals, semiconductors, and insulators, as illustrated in Figure 2.

In high-temperature superconductors, we can observe an unusual normal state which has resistivity, optical conductivity, lacks sharply defined quasiparticles, and Nernst effect. The mechanism of superconductivity at high temperatures seems to 


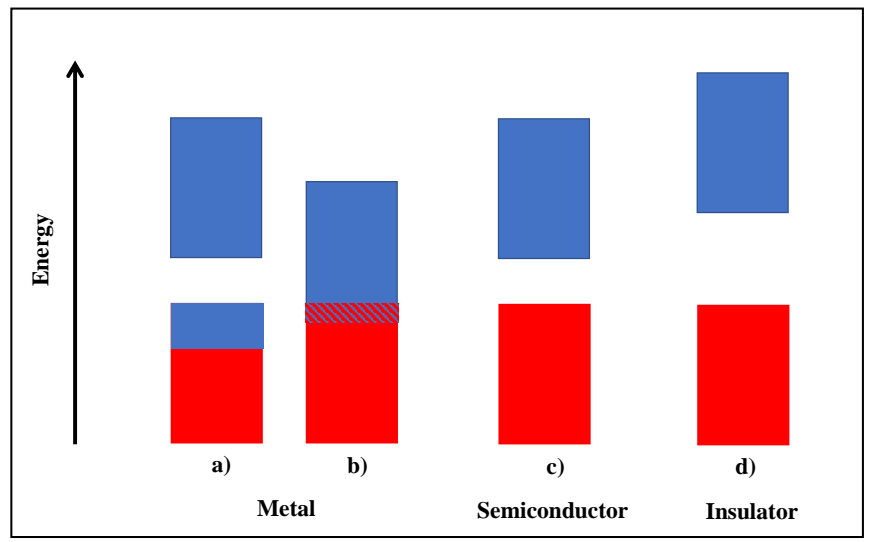

Figure 2. Classification of materials based on their band structure

be related to electron-electron and electron-phonon interactions. We cannot use the standard model of band theory to describe novel quantum materials like SCESs. To describe strongly-correlated particles in a tight-binding lattice, the Hubbard model is the standard model to [24]. The Hubbard Hamiltonian (HH) offers a simple way to describe how the interactions between electrons.[25] (see Figure 3).

Strong correlations can have a significant effect on material properties. The interplay of electron-electron, spin, charge, orbital momentum, and symmetry can drive these materials to exhibit unusual ordering, especially at low temperatures. This makes SCES very sensitive to small changes from external parameters. Those effects can be a vast increase in resistivity through a metal-insulator transition. In heavy fermion systems, at low temperatures, electron mobility behaves as if electrons are very massive particles. Some SCES exhibit a tremendous thermoelectric response. SCES displays exciting phenomena such as conventional superconductors, high $T_{c}$ superconductors, magnetic systems (ferromagnetism, antiferromagnetism), low-dimensional electron systems, quantum Hall systems, and heavy fermions systems. In iron-based superconductors (FeScs), the superconductivity pairing may be 


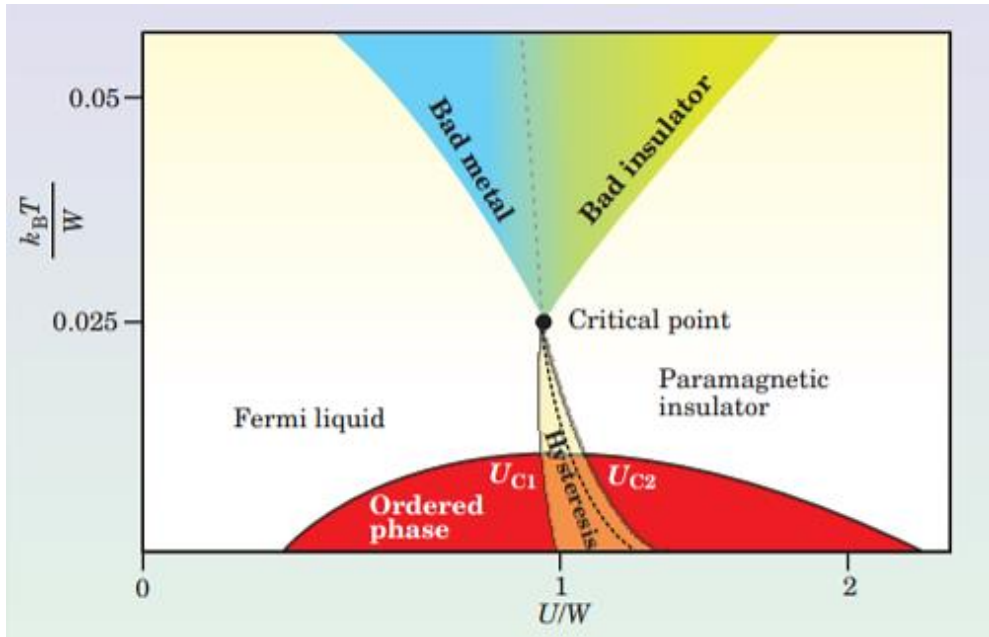

Figure 3. Mott-insulator transition phase diagram based on the Hubbard model. The strength of Coulombic repulsion $U$ and the temperature are plotted based, on the Hubbard model. At low temperature, the system had a long-range order (red). In the orange region, the model has two distinct solutions bounded by lines $U_{C 1}$ and $U_{C 2}$, the dotted line indicates first order transition in equilibrium. The first order transition line terminates at a second order critical point. The Figure is from reference [2].

due to unconventional electron-electron Coulomb interactions. Applications of superconducting material are vast and include magnetic resonance imaging (MRI), nuclear magnetic resonance (NMR), beam-steering and focusing magnets used in particle accelerators, magnetic fusion device, electric power transmission lines, magnetometer for superconducting quantum interference devices (SQUIDs), electric motors and generators, fast digital circuits, $\mathrm{RF}$ and microwave filters, superconducting nanowire single-photon detector, fast fault current limiters, and the superconducting tunnel junction detector $[26,27]$.

Iron-based superconductors share a common crystal structure with a planar layer of iron atoms centered in a tetrahedrally stacking sequence of coordinated pnictogen or chalcogen anion separated by a layer of (alkaline earth or rare earth) and (oxygen or Florine). It is believed that the organization of these layers play an important role in high $T_{c}$ superconductivity since it is similar in superconducting cuprates (Figure 1) [1]. In both FeSCs and cuprates superconductors, the doping or chemical substitution is 
an important factor to induce the superconducting phase transition.

The physics behind FeSCs is far richer than initially anticipated in that they display exceptional and highly significant properties. Understanding the phases and states that lead to the superconducting phase in FeSCs will help to understand these complex materials. One of the phases that show in the iron pnictide phase diagram is the Mott localization. The electron correlations are associated with bad-metal behavior and magnetism, which can be the reason for the high $T_{c}$ in FeSCs [28]. Studying Mott insulating behavior is important in understanding the process and explaining superconductivity in FeSC materials.

The free electron model, where electrons move freely in metal, can explain many physical properties in metals such as Ohm's law and the electrical and thermal conductivity. In a Fermi gas, at $T=0$, particles will fill the lowest energy states up to The Fermi energy $E_{F}$ occupying a radius of $K_{F}$. At temperature $T \neq 0$, some of the states will be thermally excited. The energy available for these excitations is $k_{B} \mathrm{~T}$, and the only possible excitations are from filled to unfilled electronic states. In order to include the electron-electron interactions and their effects, a material needs to be described using the Landau theory of a Fermi-liquid which assumes that momentum and spin number are good numbers to describe the quasiparticles, the electron-electron interaction can be turned on over some time $(t)$, and the resulting excitation my be may be described as quasiparticles with lifetimes $\gg t$.

The BCS theory, which is the conventional theory to explain superconductivity, is based on the fact that a net interaction between electrons for transitions is less than the phonon energy $\hbar \omega$. In a superconducting phase, this interaction is favorable over the repulsive screened Coulomb interaction. This theory explains many conventional superconducting properties like second-order phase transition at the critical temperature $T_{c}$, the specific heat variation near the absolute temperature, the Meissner-Ochsenfeld effect. Despite the successes of the BCS theory failed to ex- 
plain the unconventional high temperature superconductivity which remain not fully understood to date.

\subsection{Mott Insulators}

After the discovery of high $T_{c}$ superconductors in cuprates by Bednorz and Muller in 1986, Anderson proposed that those materials are doped Mott-insulators, which generated a great interest in understanding them.

Mott insulators are named after its discoverer Sir Nevill Francis Mott, who shared the 1977 Nobel prize with Philip W. Anderson and J. H. Van Vleck, for his contribution for his work on the electronic structure of magnetic and disordered systems, especially amorphous semiconductors. Mott insulators can be defined as systems with strong electron-electron interactions that drive a quantum phase transition from a metallic phase to an insulating ground state [29]. Mott insulators are used in thin-film magnetic heterostructures, low power memory, thermal sensing, Field-Effect Transistors (FET), switches, junction Mott Transition Field-Effect Transistor (JMTFET), and high $T_{c}$ superconductivity applications [30, 31, 32].

Novel physics often appears near a Mott quantum paramagnetic transition (QPT) and the type of this order, whether it is first-order or second-order, is important. The nature of these transitions, and quantum fluctuations near them, are of great interest because they help in understanding high $T_{c}$ iron superconductors [33]. The mechanism of Mott insulator transition is not fully explained; however, many current scientific suggestions explain it by the opening of charge-transfer gap, orbital ordering, and charge ordering, but no experimental evidence to support these possible explanations has been found [33]. In the Mott insulating state, the charge degrees of freedom are frozen, and the associated spin degrees of freedom are considered as local moments. These local moments are coupled with each other through exchange interactions and frequently behave as an antiferromagnetic order [34]. The Mott-Hubbard transition is 
one of the most spectacular direct effects of strong correlations and its understanding is believed to be essential to understand the behavior of FeSCs. The Mott-Hubbard transition is simply the process connecting a metallic state with a Mott-Hubbard insulator, a state in which the electronic conductions are inhibited by strong local Coulomb repulsion despite the partial filling of the band [35].

The iron oxychalcogenides crystal structure contains tetragonal symmetry with planes containing iron $\mathrm{Fe}$ and oxygen $\mathrm{O}$ atoms for which chalcogens alternate above and below those planes. Figure 1 shows the planer iron atom Fe arrangement in FeScs which is similar to the arrangement in iron oxychalcogenide materials [9]. Iron oxychalcogenides such as $\mathrm{X}_{2} \mathrm{O}_{2} \mathrm{Fe}_{2} \mathrm{O}_{2}$ (with $\mathrm{X}=\mathrm{Ca}$, La and $M=\mathrm{S}$, Se) are an important family to investigate. Iron oxychalcogenides are structurally related to the superconductors and because the substitution of the chalcogenide ion tunes the electronic bandwidth hence their proximity of a metal-insulator transition [36].

\subsection{Iron-based Superconductors}

Iron-based superconductors (FeSCs) are interesting; they have high critical temperature $T_{c}$, and in these materials, the electrons seem to form cooper pairs via a Coulombic interaction without help from the ionic lattice [37]. The need to understand the pairing mechanism in the FeSCs had driven research into these materials. FeSCs material includes many iron pnictides and iron chalcogenides. Figure 1 shows the crystal structure of some pnictide and chalcogenide iron superconductors. The

square lattice iron atom planer layer is shown on the right side of Figure 1, this layer is very common among FeSCs material structure. In iron-based superconductors, the superconductivity pairing may be due to unconventional electron-electron Coulomb interaction. Two distinctive features of iron-based materials are their complex phase interplay and a surprisingly large chemical variety. The presence of high $T_{c}$ superconductivity in doped Mott insulators such as the cuprates contradicts the 
conventional thought that electron repulsion is detrimental to the presence of superconductivity. Nematicity has been the focus of research and studies in the field of iron superconductors during the last decade. It leads to anisotropy in the magnetic properties, electric transport, orthorhombic structural distortions, and changes in the band splitting close to the Fermi level [38]. In the nematic phase (state) three different order parameter is present (non zero) and can be classified into the following three types: 1. structural distortion 2. charge/orbital order and 3. spin order [17]. Structural nematicity is short-range (local) fluctuations with or without a structural phase transition. Nematicity can be considered translational invariant even though it spontaneously breaks rotational symmetry. In many strongly correlated electron systems with very small structural anisotropy in the plane, such as cuprates and pnictides, ordered states of matter are characterized by a broken symmetry according to the Landau paradigm of continuous phase transitions [39]. Nematic ordering breaks the tetragonal symmetry of iron-based superconductors. It emerges from a regular structure transition of electric instability in the non-superconducting (normal) phase [20]. The susceptibility of the iron pnictides superconductor $\mathrm{Ba}\left(\mathrm{Fe}_{1-x} \mathrm{Co}_{x}\right)_{2} \mathrm{AS}_{2}$ distinguished an electric nematic state which is near the superconducting transition temperatures [39].

The presence of nematic order in iron-based superconductors is a well-established fact experimentally, although its origin remains controversial. Nematicity order state can happen because of a regular structural transition or as the result of an electronically driven instability in particular orbital order or spin-driven Ising-nematic order. The Ising-nematic order is a magnetic state that breaks rotational symmetry but preserves time-reversal symmetry. Symmetry dictates that the development of one of these orders immediately induces the other two, making the origin of nematicity a physics realization of the 'chicken and egg problem' [17]. 


\subsection{Mott insulators in this study}

New materials that exhibit strong correlations are continuously being developed. Recently the $\mathrm{Ca}_{2} \mathrm{O}_{2} \mathrm{Fe}_{2.6} \mathrm{OS}_{2-x} \mathrm{Se}_{x}$ has been synthesized and there are limited studies on its nuclear and magnetic structure. In addition, some materials like $\mathrm{La}_{2} \mathrm{O}_{2} \mathrm{Fe}_{2} \mathrm{O}(\mathrm{S}$, $\mathrm{Se})_{2}$ lacks studies on the local structure using the Pair Distribution Function (PDF) analysis. In this work, both x-rays and neutrons scattering techniques were employed to investigate the behaviors of strongly correlated electronic systems. Mott insulating iron oxychalcogenides $\mathrm{Ca}_{2} \mathrm{O}_{2} \mathrm{Fe}_{2.6} \mathrm{OS}_{2-x} \mathrm{Se}_{x}$ and $\mathrm{La}_{2} \mathrm{O}_{2} \mathrm{Fe}_{2} \mathrm{O}(\mathrm{S}, \mathrm{Se})_{2}$ in proximity to superconductors were studied. The nuclear structure of the newly reported $\mathrm{Ca}_{2} \mathrm{O}_{2} \mathrm{Fe}_{2.6} \mathrm{OS}_{2-x} \mathrm{Se}_{x}$ was studied to search for phase transition as well as lattice parameter temperature dependence to understand how atomic structure is related to the electronic and magnetic properties. Also, further analysis of $\mathrm{Ca}_{2} \mathrm{O}_{2} \mathrm{Fe}_{2.6} \mathrm{OS}_{2-x} \mathrm{Se}_{x}$ magnetic structure and estimation of the order parameter (magnetic phase) was performed due to the unavailability of published data. Furthermore, we investigated the local structure of $\mathrm{La}_{2} \mathrm{O}_{2} \mathrm{Fe}_{2} \mathrm{O}(\mathrm{S}, \mathrm{Se})_{2}$ by the neutron total scattering technique and the Pair Distribution Function (PDF). 


\section{CHAPTER 2}

\section{LOW-DIMENSIONAL SYSTEMS}

In the past ten years, satisfying Moore's law has required the adoption of new materials and new device geometries in order to continue improved performance. Two-dimensional materials (2DM) have been studied intensely for potential application are one of the most studied classes of materials due to the richness of unusual physical phenomena that occur when charge and heat transport is constrained in a plane. Many materials show special properties due to reduced dimensions, such as the layered transition metal dichalcogenides (TMDs), copper oxides, and iron pnictides. All of these systems which exhibit correlated electronic phenomena as varied as charge density waves and high-temperature superconductivity.

The isolation of graphene by Andre Geim and Konstantin Novoselov in 2004, from bulk graphite, has led to a tremendous focus on other 2DMs such as transition metal dichalcogenide. One of the challenges that face the 2DMs, to be used in devices and high volume manufacturing, is to grow large and high-quality single crystals. Many 2DMs crystals are now beginning to be investigated. There are hundreds of layered materials that hold their stability to the monolayers, having properties complementary to graphene. Transition metal oxides (TMOs) and transition metal dichalcogenides (TMDs) have a layered structure. In both TMOs and TMDs, atoms within layers are held together by covalent bonds, whereas different layers are held by van der Waals interactions. TMDs offer advantages in optoelectronics due to their transparency, mechanical flexibility, easy processing, and the ability to tune the bandgap by varying the number of layers that allows the detection of light at differ- 
ent wavelengths. TMDs properties complement graphene's properties. For example, graphene is useful for broadband ultra-fast technologies, while semiconducting TMDs are better for applications requiring strong light absorption and electroluminescence. The Combined properties of both graphene and TMDs can be used to create multifunctional and high-performance materials that consist of heterogeneous stacks and hybrid devices [40]. 2DM are important in various applications such as optoelectronics, spintronics, catalysts, chemical, and biological sensors, supercapacitors, solar cells, digital circuits, light-emitting diodes, and lithium-ion batteries [3].

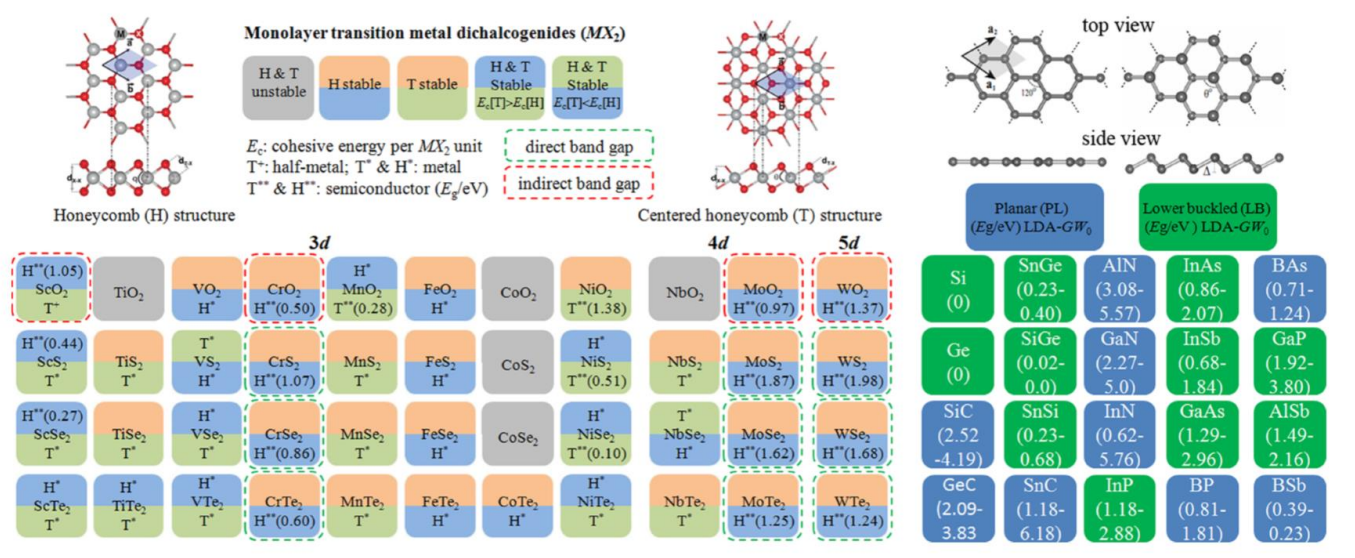

Figure 4. Different $\mathrm{MX}_{2}$ compounds and binary compounds of group-IV elements and group III-V. The structure ( $H$ or $T$ ) is the ground state. The resulting structures $(T$ or $H)$ can be half-metallic $(+)$, metallic $\left(^{*}\right)$, or semiconducting $(* *)$ with direct or indirect band gap [3].

\subsection{Weyl Semi Metals}

In condensed matter physics, the intersection of topology and the band theory has recently attracted the attention of researchers. The first topological material to show these effects was Weyl semimetals (WSM) [41]. Topological Weyl semimetals 
(WSM) can be classified as either type-I WSM, which has a vanishing density of states at the Weyl nodes or type-II WSM, which have singular points of momentum space allowing for distinct topological properties. In 1929 Hermann Weyl proposed that the existence of Weyl fermions were massless chiral fermions in his paper "Gravitation and the Electron" [42], and he proposed a solution to Weyl equation derived from the Dirac equation. Weyl fermions as quasiparticles in a low-energy condensed matter system. Such quasiparticles were discovered in 2015, in the form of crystals known as Weyl semimetals, as a type of topological material.

Weyl semimetals are a gapless topological phase protected by the chiral anomaly, where the charge and the crystal translational symmetry are conserved. The absence of a bandgap in a weakly-interacting Weyl semimetal is sustained by the electronic structure topology and will remain intact as long as the symmetries and the anomaly are intact. This nontrivial topology also establishes in the Fermi arc surface states and topological response [43]. Weyl semimetal is the first example of a bulk gapless topological phase [41]. The vanishing gap of the bulk electronic structure in WSM is sustained by topology. The existence of closed surfaces in momentum space that carry nonzero Chern numbers creates the presence of a band-touching point inside the Brillouin zone (BZ) volume. In order to have a separation between the Weyl nodes in momentum space, an inversion or a time-reversal (TR) symmetry needs to be violated while maintaining the crystal translational symmetry.

Weyl semimetals show identification features like linear dispersion at Weyl nodes (or Weyl points), monopoles of Berry curvature originating at the location of the Weyl points in momentum space, and unique boundary modes known as Fermi arcs that connect projections of these Weyl nodes in the surface Brillouin zone [44]. Figure 5 illustrate the Weyl nodes (points) in WSM material. Type-I WSM, in which the density of states vanishes at the energy of the Weyl nodes, shows many essential physics properties like thermoelectric properties, [45] electro- and magneto-transport [46], 
magnetic properties [47], and effect of disorder [48]. In type-I WSM, when the chemical potential is shifted slightly away from the nodal energy, the Fermi pockets enclosing the projections of the Weyl nodes are very small. In contrast, type-II WSM has an extended pocket of holes and electrons at the node energy. Doping away from the node energy will results in surface projections of the Weyl nodes becoming bounded within large Fermi pockets.

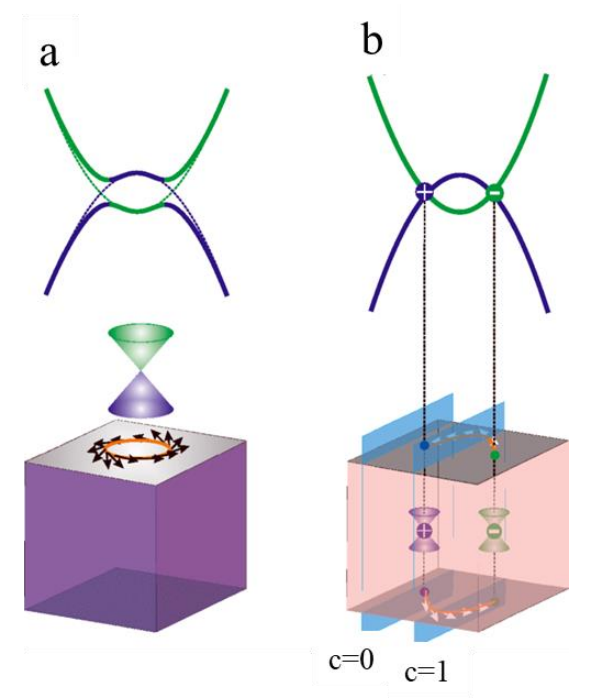

Figure 5. Schematics of the topological insulator and Weyl semimetal [4].

\subsection{Transition Metal Dichalcogenide}

In 1923, the crystal structure of molybdenite monolayers was studied by Dickinson and Pauling, and monolayer $\mathrm{MoS}_{2}$ was investigated in 1986. Transition Metal Dichalcogenide (TMD) materials have the advantage over graphene of having extended bandgap tunability through composition, thickness, and possibly even strain control [49]. TMDs consist of hexagonal layers of metal atoms (M typically Mo, W, $\mathrm{Nb}, \mathrm{Re}, \mathrm{Ni}$, or $\mathrm{V}$ ) pack in between two layers of chalcogen atoms (X typically $\mathrm{S}, \mathrm{Se}$, or $\mathrm{Te}$ ) with a $\mathrm{MX}_{2}$ stoichiometry which gives more than 40 different combinations for TMDs as in figure 4. The bulk 3D crystals are stacked structures with van der 
Waals bond between adjacent layers with strong covalent bonding within each layer. TMDs shows a range of electronic structures from insulators to metals with interesting properties including topological insulator effect [50], thermoelectricity [51], and superconductivity [52]. TMDs established themselves as fascinating building blocks for optoelectronic applications, with a strong focus on various photodetection platforms. The adaptability of TMDs enables their application in areas including ultrafast and ultrasensitive detection of light in the ultraviolet, visible, infrared and terahertz frequency ranges [40] which can be used in photonic components. Bulk TMDs are one of the most interesting classes of materials. They display a vast range of interesting properties such as semiconductivity, half-metallic magnetism [53], superconductivity [54], or charge density wave [55]. These properties made TMDs candidate for many applications in numerous areas, including lubrication, catalysis, photovoltaics, supercapacitors, and rechargeable battery systems [3]. Multi-level $\mathrm{MoTe}_{2}$-based resistive random-access memory (RRAM) devices with a new switching mechanism and speeds of less than five ns due to an electric-field induced $2 H$ to $2 H_{d}$ phase transition. These devices offer fundamentally better reliability and control for faster switching. $\mathrm{MoTe}_{2}$-based RRAM devices allow faster switching due to a transition between two crystalline states induced by an electric field. The phase transition from the stable semiconducting $2 H$ phase to a more conductive $2 H_{d}$ phase provides a potential path towards better stability [56].

Density functional theory (DFT) calculations showed for bi-layer $\mathrm{MoS}_{2}, \mathrm{WS}_{2}$, $\mathrm{MoSe}_{2} 2$, and $\mathrm{MoTe}_{2}$ that their fundamental indirect band gaps can be driven to zero by applying external electric field perpendicular to the layers which result in a gap tunability is much larger than in bi-layer graphene [3].

From studying strain effects on the electronic structures of monolayer $2 \mathrm{H}-\mathrm{MX}_{2}$ sheets where $\mathrm{M}=\mathrm{Mo}$ or $\mathrm{W}$ and $\mathrm{X}=\mathrm{S}$, Se or Te by using first-principles calculations, Yun et al. [57] found that even a small different lattice value from the bulk optimum 
value could induce the direct-to-indirect bandgap transition, change the gap energy (the positions of the valence band maximum and the conduction band minimum) of the single-layer sheets. The ability of tuning $\mathrm{MoTe}_{2}$ can affect its topological properties since the Weyl points are sensitive to changes in the lattice parameter of the $\mathrm{MoTe}_{2}$ crystal.

\subsection{Low-dimensional materials in this study}

Low-dimensional materials have the potential to be used in a wide range of electronic devices due to their unique properties. In the current research, we evaluated the change in low-dimensional material structures with the change in temperature. We studied the structure in low-dimensional topological materials that show TypeII Weyl semimetal behavior in order to assess their viability as topological phase switches. The powder neutron and x-ray diffraction were used to estimate the phase transition and the coexistence range. Also, we evaluate the temperature dependence of the lattice parameters in $2 H$ and $1 T^{\prime}$ phases of $\mathrm{MoTe}_{2}$. 


\section{CHAPTER 3}

\section{SCATTERING THEORY}

\subsection{Introduction to Scattering}

The interaction between a beam of particles and a material will depend on the energy, charge, and spin of the incoming probe. Figure 6 shows how incoming x-ray and neutron beams scatter from a condensed matter sample.

a)

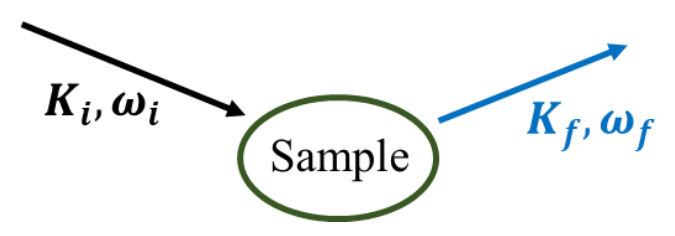

b)

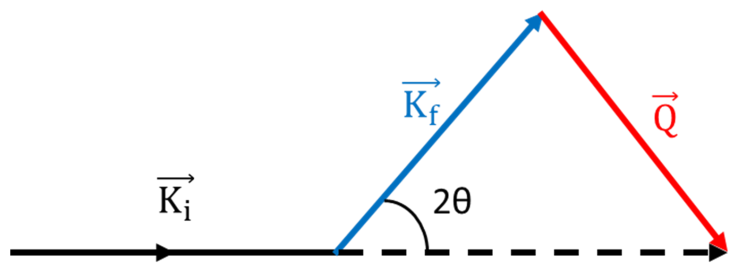

Figure 6. Scattering process of a particle scattering from a sample in a) and b) shows the scattering vector $\vec{Q}$. This relation holds for any type of particles. In elastic scattering $\left|K_{i}\right|=\left|K_{f}\right|$, while for inelastic scattering $\left|K_{i}\right| \neq\left|K_{f}\right|$.

From conservation of momentum we can obtain the transfer momentum $\vec{P}$, by:

$$
\begin{aligned}
& \vec{P}=\hbar \vec{K}_{i}-\hbar \vec{K}_{f}=\hbar \vec{Q} \\
& \vec{Q}=\vec{K}_{i}-\vec{K}_{f} \\
& E=\hbar \omega=\frac{\hbar^{2}}{2 m}\left(\vec{K}_{i}^{2}-\vec{K}_{f}^{2}\right)
\end{aligned}
$$

Scattered particles can be classified as elastic, inelastic, coherent, and incoherent scattering. Elastic or inelastic scattering processes are identified by energy loss. If the energy describing the scattering process remains unchanged this means that it is an elastic case, or has a definite change in energy after interaction with the sample which leads to inelastic scattering. If the scattered particles have interference with 
each other then this scattering is called coherent scattering. On the other hand it is called incoherent scattering when there is no phase relationship, and all of the scattered particles have different relative phases. X-ray, neutron, light and electrons are commonly used to provide a range of information about the studied material. The information that can be obtained depends on the interaction of the probe particle and the constituent of the material under study. Figure 7 show how x-ray and neutron interact with a sample. X-rays interacts mainly with the nucleus and this will produce information about the nuclear structure of the sample. Neutrons in the other hand interact with the nucleus and because they have a magnetic moment they also can interact with the magnetic moment of the electron to provide information about the magnetic structure of the sample.

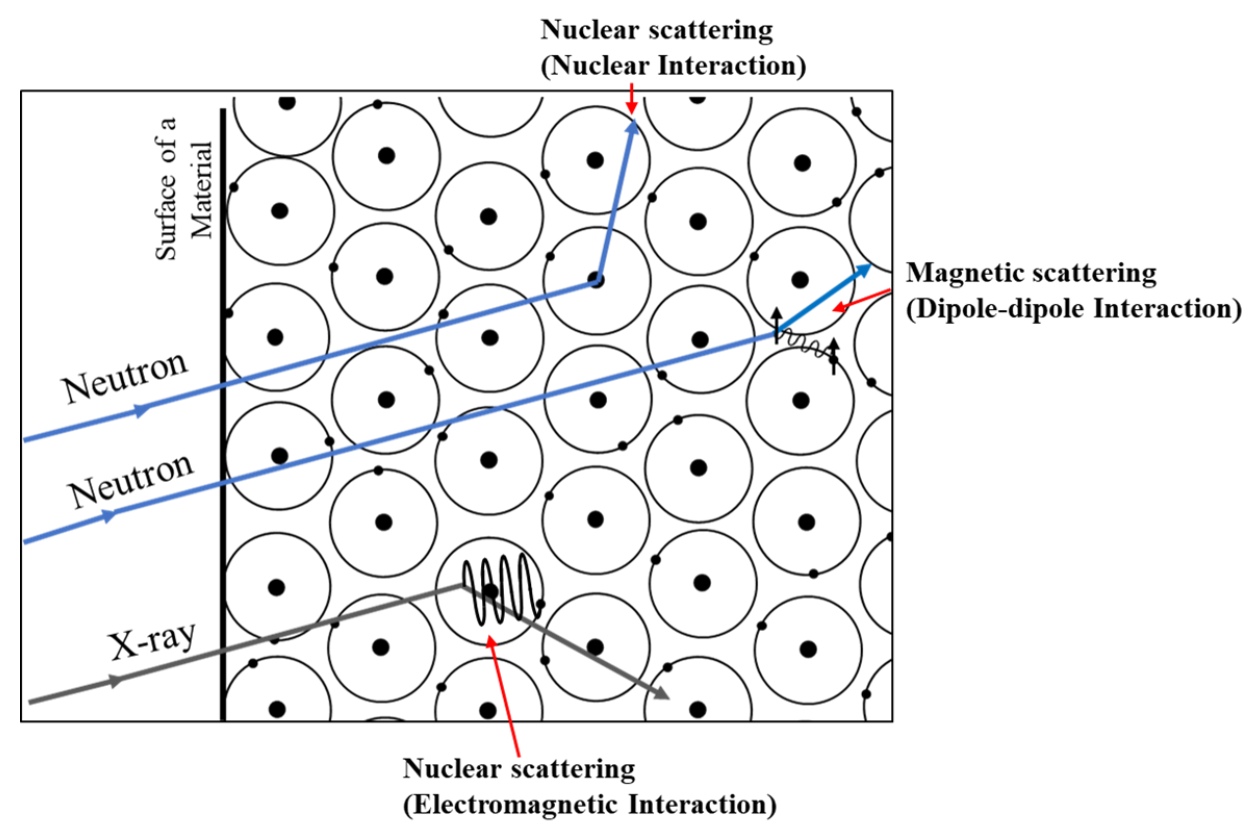

Figure 7. The figure illustrates neutron interactions with the matter which produce nuclear and magnetic scattering 


\subsection{X-Ray Scattering}

X-ray scattering is an impressive arsenal of tools that have been developed over the last century to understand the crystallography of materials. Currently, x-rays can determine the structure and provide insights into the functionality of systems from monolayers to bulk materials.

X-rays were discovered in 1895 by Wilhelm Röntegen, and since that time, it has been considered an essential tool for investigating the field of condensed matter [58]. In 1912 Max von Laue obtained the first diffraction pattern from a crystal. A year later, W.H. Bragg and W.L. Bragg, a father, and son, laid the foundation of crystallography [58]. This allowed the determination of crystal structure by introducing the famous Bragg formula, which describes the x-ray diffraction in terms of reflection of x-ray by lattice planes. Figure 8 illustrate Bragg law and Equation 2 states it. For an x-ray beam scattered from an electron, the scattering event exerts a force on the electron from the x-ray electric field. Then the accelerated electron radiates the scattered wave. If the wavelength of the scattered wave is the same as the incident one, then the scattering is elastic. If the scattered wavelength differs from the incident one, then the scattering is inelastic, and this is also known as the Compton effect $[58]$.

$$
n \lambda=2 d \sin \theta
$$

The x-ray scattering intensity $I$ is proportional to how efficient the particle in the sample scatter the radiation. This is given by the differential cross section $\left(\frac{d \sigma}{d \Omega}\right)[58]$.

$$
I \propto\left(\frac{d \sigma}{d \Omega}\right)
$$

The differential cross section is defined by:

$$
\left(\frac{d \sigma}{d \Omega}\right)=\frac{\text { Number of } x-\text { rays scattered per second into } d \Omega}{(\text { incidentflux })(d \Omega)}
$$




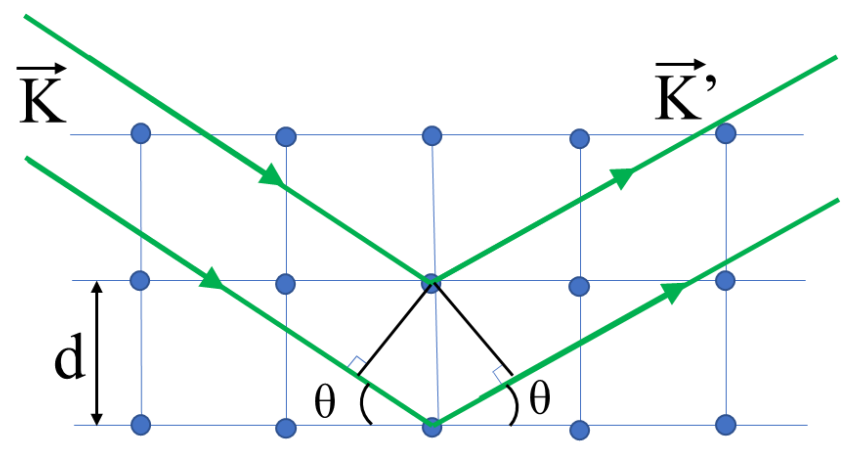

Figure 8. Illustration of Bragg Law, $\vec{K}$ is the incoming wave, $\vec{K}^{\prime}$ is outgoing wave, and $d$ is the interatomic spacing.

According to Nielson and McMArrow [58] the differencial cross section for x-ray yields to:

$$
\left(\frac{d \sigma}{d \Omega}\right)=r_{o}^{2} P|F(\vec{Q})|^{2} N \frac{\lambda^{3}}{v_{c}} \frac{1}{\sin 2 \theta}
$$

Where $r_{o}$ is the Thomson scattering length, $P$ the polarization factor, $N$ is the total number of unit cells, $\lambda$ is the incident beam wavelength, $v_{c}$ is the volume of unit cell, $\theta$ is the integrated angle and $\frac{1}{\sin 2 \theta}$ is called the Lorentz factor. $F(\vec{Q})$ is the structure factor which is defined as:

$$
F(\vec{Q})=\overbrace{\sum_{j} f_{\text {atomic }}(\vec{Q}, \lambda) e^{i \vec{Q} \cdot \vec{r}}}^{\text {Unit cell }} \overbrace{\sum_{n} e^{i \vec{Q} \cdot R_{n}}}^{\text {Lattice }}
$$

Where $\overrightarrow{R_{n}}$ is the lattice vector, $\overrightarrow{r_{j}}$ is the position of an atom within the unit cell, and the first term sum is over the basis of the atom and the second term is over the lattice. Using $\vec{Q}=\frac{\sin \theta}{\lambda}$, the atomic form factor is defined as:

$$
f_{\text {atomic }}(\lambda, \theta)=f_{o}\left(\frac{\sin \theta}{\lambda}\right)+f^{\prime}(\lambda)+i f^{\prime \prime}(\lambda)
$$

Where $f_{o}\left(\frac{\sin \theta}{\lambda}\right)$ is The atomic form factor that express the charge spacial profile inside the atom at high energies and is obtained by Fourier transform of the electron density [59]. The terms $f^{\prime}(\lambda)$ and $i f^{\prime \prime}(\lambda)$ are the atomic forwarding scattering factor (also called dispersion corrections) and their values depend strongly on $\lambda$. The scattering form factor for x-rays is shown in Figure 9. For the x-ray scattering, the form factor 
diminishes monotonically with increasing $\theta$ and decreasing $\lambda$ (as a function of $\frac{\sin \theta}{\lambda}$ ), has the same sign for all element and has a magnitude that is proportional to the atomic number $Z$ of the scatterer atom.

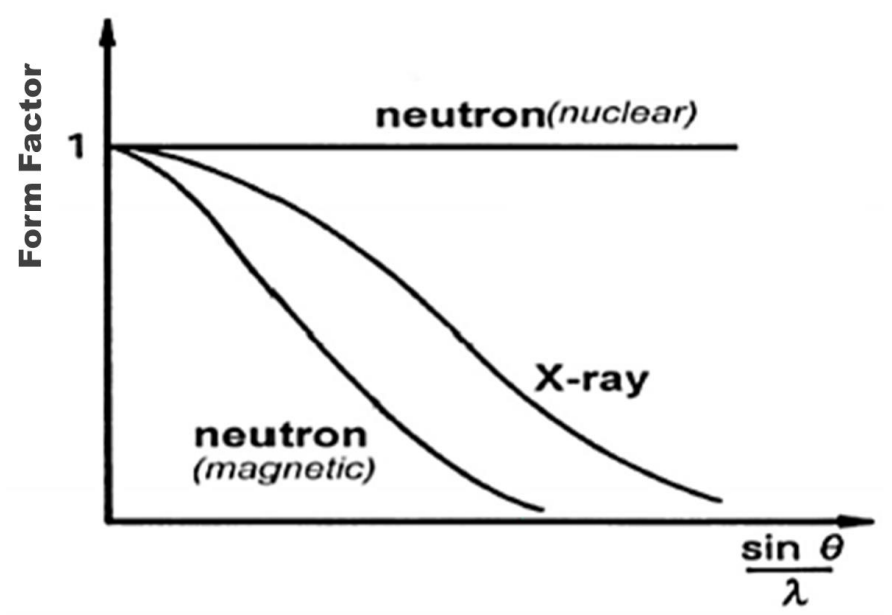

Figure 9. Comparison between x-ray and neutron form factors. All form factors are normalizes to unity at $\frac{\sin \theta}{\lambda}=0$.

A common technique known as the Laue method uses a broad spectrum of x-rays. The Laue method is historically the oldest x-ray technique and was used in the original discovery of x-ray diffraction. Figure 10 illustrates Bragg and Laue diffraction. The Laue diffraction is used to orient single crystals and to determine their crystal quality. However, with the advent of synchrotron radiation and enormous improvements in computing power and detector technology, Laue diffraction has maintained a renaissance as a technique that provides a fast and efficient means to record diffraction data and is being used to significant effect in dynamical studies of transient crystalline states. One of the advantages of the synchrotron source is producing intense, brilliant photon beams on the very smallest of samples, which had a significant positive effect on the research in condensed matter physics. These x-rays beams can probe the inter-atomic distances in materials. 


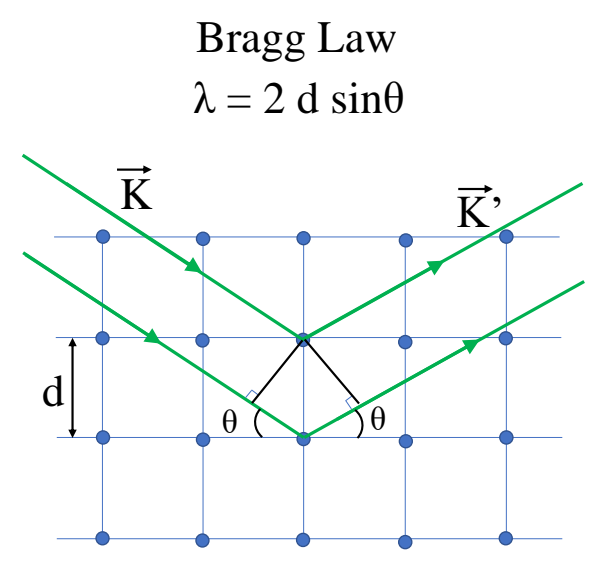

Real Space
Laue Condition $\overrightarrow{\mathrm{Q}}=\overrightarrow{\mathrm{G}}$

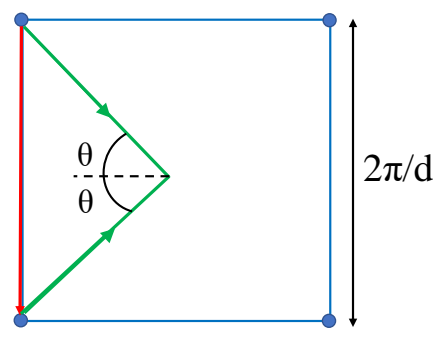

Reciprocal Space

Figure 10. Comparison between Bragg and Laue scattering.

\subsubsection{X-Ray Production}

X-rays, which are commonly used for structural determination, can be obtained in labs by a benchtop machine or in a synchrotron. Although lab x-rays are beneficial tools and convenient for performing qualitative and quantitative studies, the quality of the data collected from a synchrotron beamline is of a high-resolution. In the early days of synchrotrons, x-rays were an undesirable by-product in high-energy particle accelerators facilities. In modern synchrotrons, electron beams are accelerated to speeds close to the speed of light, which generates the emission of bremsstrahlung radiation, which is emitted when the electron beams path is curved by magnetic fields. The sources of electrons in synchrotron are electron guns where cathode heated to $1000^{\circ} \mathrm{C}$ to produce a beam of electrons. Electrons are accelerated in an evacuated linear accelerator (LINAC) and then enter a booster ring where electron energy is boosted to the desired energy of the storage ring, which is in the range of $\mathrm{GeV}$. In the storage rings, which can span over a few hundred meters of circumference, radiation is produced in the bending magnets. Bending magnets or undulators which are in the straight section of those rings. Electrons trajectories are controlled by 
magnetic fields. A radio frequency $(\mathrm{RF})$ source restores the energy to the beam on each subsequent pass. The x-ray radiation is then directed to beamlines. A beamline is the trajectory of the x-ray beam and the instrumentation that directs the beam towards the experimental assembly area. Each beamline is designed for a specific technique or a specific type of research. Figure 11 shows the different parts of a synchrotron and all beamlines at the Advanced Photon Source (APS) of the Argonne national laboratories, IL.

\subsubsection{Synchrotron Beamline Used in This Research}

\subsubsection{Advanced Photon Source (APS) 11-BM}

A dedicated high-resolution, high-throughput powder diffraction beamline, 11-BM is located at the Advanced Photon Source (APS) at Argonne National Laboratory. 11-BM is intended to facilitate high-resolution crystallographic structure determination from powder diffraction of complex polycrystalline materials. 11-BM supports a wide range of user interests, from pharmaceuticals through traditional materials science to questions in engineering, catalysis, chemistry, geosciences, physics, and electronics [60].

The x-ray beam size at the sample is $\approx 1.5 \mathrm{~mm}$ (horizontal) $\mathrm{x} 0.5 \mathrm{~mm}$ (vertical). The focused beam efficiently utilizes the bending magnet source and minimizes asymmetric diffraction peak broadening from axial divergence even at low angles. The 11-BM beamline couples an efficient focused x-ray beam with a high precision diffractometer circle and perfect $\mathrm{Si}(111)$ crystal analyzer detection to achieve high sensitivity and resolution. Instrumental resolution at high $Q$ is better than $\Delta Q / Q \approx 2 \times 10^{-4}$, with a typical $2 \theta$ resolution of $<0.01^{\circ}$ at $30 \mathrm{keV}$. For mail-in samples, a Kapton capillary tubes will be supplied to the user. These capillary tube inner diameter of 0.80 mm, and an outer diameter that fits snugly inside 11-BM mounting bases. Figure 12 shows 11-BM mail-in Kapton capillary tubes. The capillary holding measured sample 


\section{ARGONNE NATIONAL LABORATORY 400-AREA FACILITIES ADVANCED PHOTON SOURCE \\ (Beamlines, Disciplines, and Source Configuration)}

\section{ADVANCED PROTEIN CHARACTERIZATION FACILITY CENTER FOR NANOSCALE MATERIALS}

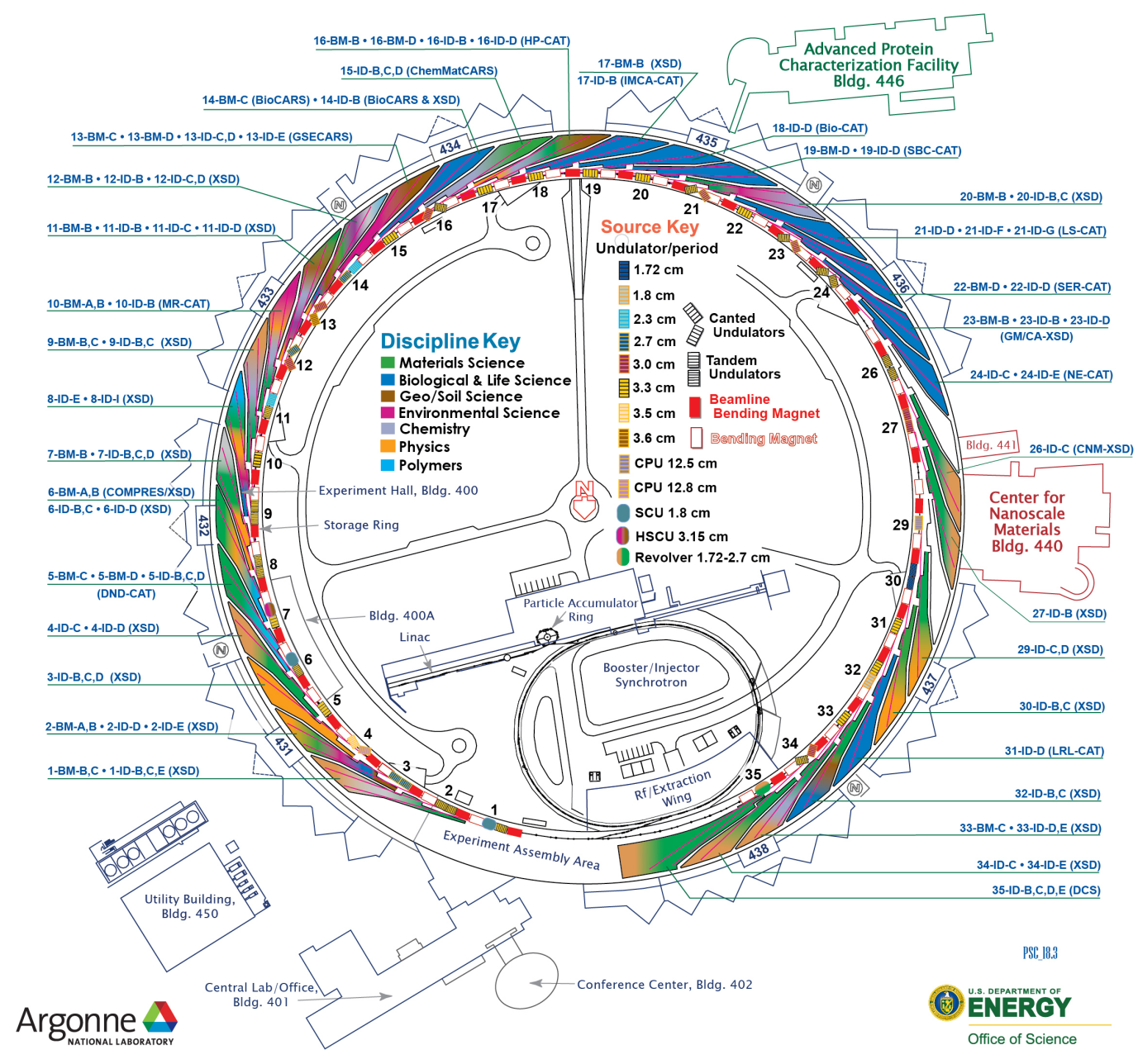

Figure 11. APS plan view shows every beamline and the different parts of the synchrotron from https://www.aps.anl.gov/Beamlines 


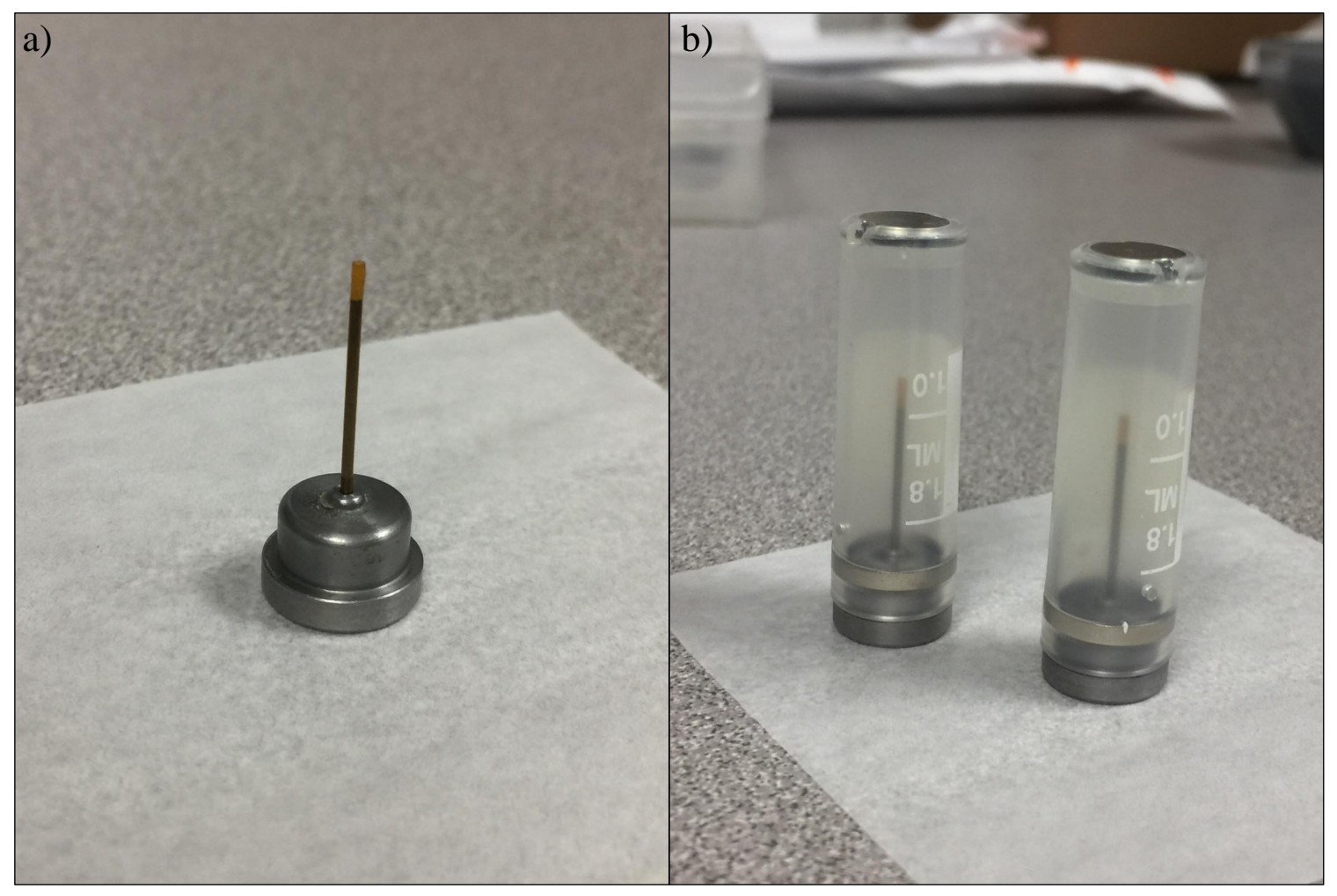

Figure 12. 11-BM sample holder consists of a mounting base, a magnetic cap, and a capillary tube. The mounting base he a unique ten-digit barcode to tracks the sample at all steps of our mail-in service. A magnetic ring secures caps on mounting bases and provides some protection for sample capillary tubes during shipment. The Kapton tubes have an inner diameter of $0.80 \mathrm{~mm}$, and an outer diameter that fits snugly inside 11-BM mounting base. On the left, the base and the tube filled with a sample is shown. On the right, the picture shows samples bases and capillary tubes, with the protective magnetic cap.

contributes a weak background to the total sample scattering. 


\subsection{Neutron Scattering}

Neutrons were discovered by James Chadwick in 1932. Neutrons are subatomic particles with no net electric charge, a mass of $939.5656 \mathrm{MeV} / \mathrm{c}^{2}$, and spin $\frac{1}{2}$. Neutron elastic and inelastic scattering has become an important tool for the determination of atomic, magnetic structures, and magnetic excitations. Neutron scattering, nondestructive techniques, are used in biology, chemistry, condensed matter physics, metallurgy, ceramics, and polymers providing information unobtainable by any other means. Sometimes this invaluable information is complementary to that obtained by more traditional methods [61]. Some of the advantages of the neutron scattering technique are neutron wavelengths and energies that well matched to interatomic spacings and excitations in condensed matter. Figure 13 shows length and time domains achieved by neutron scattering techniques. Neutron nuclear scattering does not associate with a form factor. Neutrons carry no charge and penetrate a large volume of the sample. A major disadvantage of this technique is that the scattering length varies irregularly among elements and isotopes for nuclear scattering. Another disadvantage is that neutron scattering is intensity limited, and it is costly to produce neutron beams.

Figure 7 illustrates neutrons interaction with matter. Neutrons interact with matter in two ways. First, they interact with atoms by nuclear forces. Second, they interact with the magnetic fields of unpaired electrons through magnetic dipole interactions. Neutron penetrates deeply into most materials, which makes neutron scattering suitable as a bulk probe.

Neutrons have de Broglie wavelength $\lambda \approx 1-2 \AA$ and energies of $100-20 \mathrm{meV}$. Neutrons with these energies are called thermal neutrons. They are excellent probes of low-energy excitations such as lattice vibrations and spin waves with energies in the meV range. Neutron beams can be produced by fission reaction in a reactor or by spallation due to collisions of high-energy protons with a heavy metal target. Neutron 


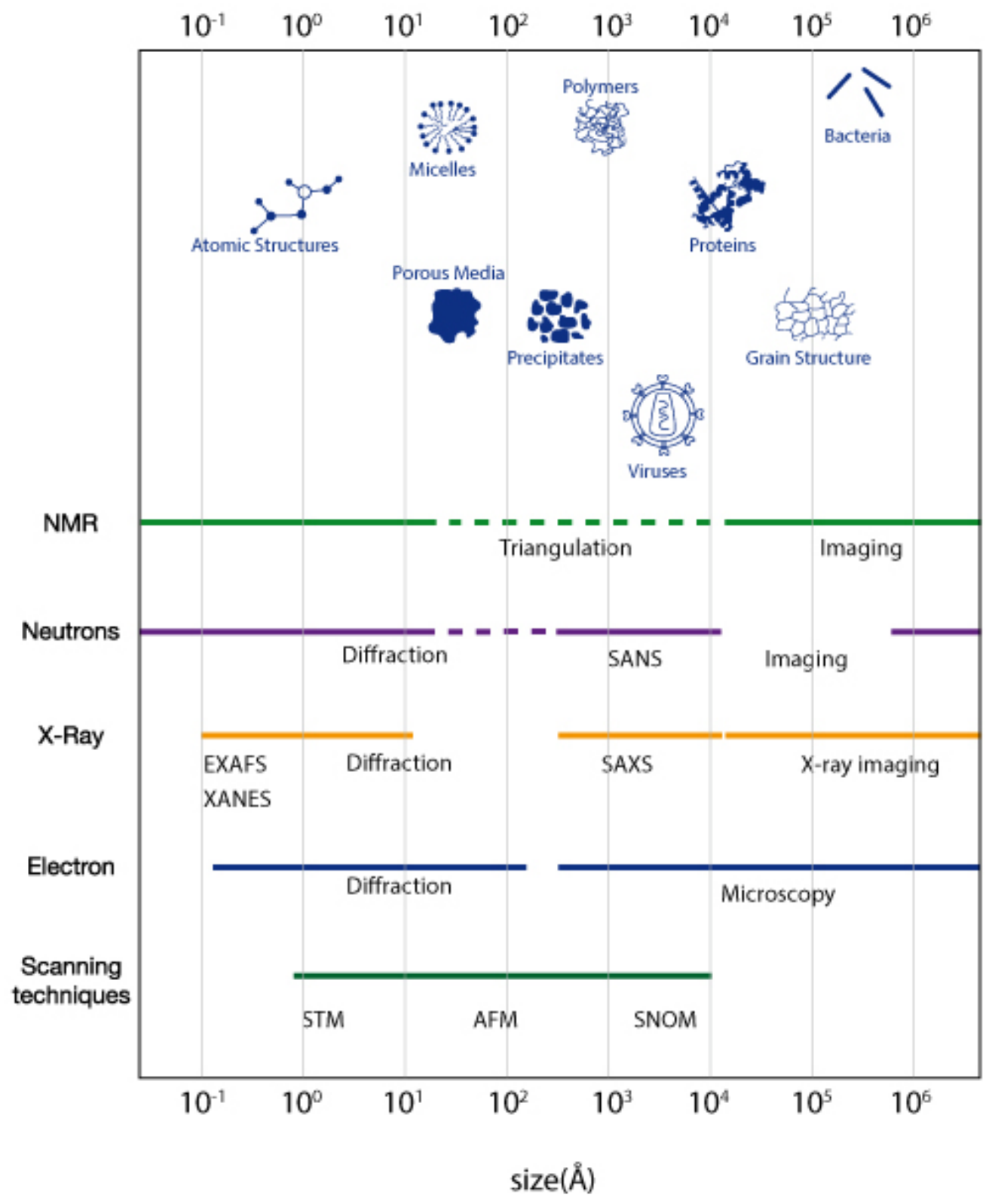

Figure 13. Length and time domains available with neutron scattering techniques compared to other investigation methods. Source https://mlz-garching.de/ englisch/neutron-research/experimental-methods/elastic-scattering.html

scattering depends on the scattering length and not the atomic number. This results in light elements being highly detectable, and adjacent elements in the periodic table being easily distinguishable. 


\subsubsection{Nuclear Neutron Scattering}

Travelling neutrons have a wavelength given by de Broglie equation:

$$
\lambda=\frac{h}{m_{n} v}
$$

Where $m_{n}$ is the neutron mass, and $h$ is Plank constant. As the neutron approaches the atomic nucleus, it can be scattered by the nuclear forces or by the spin-spin interactions. The magnetic moment of the neutron can interact with both the nuclear magnetic moments and the unpaired electron moments. The neutron wavelength is about $10^{4}-10^{5}$ times larger than the nuclear forces range which makes the nuclear neutron scattering just like a point scattering and the scattered neutron form factor,

or scattering length $b$, for a given element is independent of $\frac{\sin \theta}{\lambda}$ (Figure 9) [59]. Similar to x-ray scattering, the nuclear neutron scattering intensity, is proportional to the differential cross-section as:

$$
I \propto\left(\frac{d \sigma}{d \Omega}\right)=\frac{\text { Number of neutrons scattered per second into } d \Omega}{(\text { incidentflux })(d \Omega)}
$$

The Analysis of scattered neutrons from a random distribution of scatterer lead to the following expression for the differential cross section $[62,5]$.

$$
\frac{d \sigma}{d \Omega}=|\bar{b}|^{2}\left|\sum_{n} \exp \left(i \vec{Q} \cdot \overrightarrow{r_{n}}\right)\right|^{2}+N \overline{|b-\bar{b}|^{2}}
$$

Where $\bar{b}$ is the mean scattering length for all atoms, $\vec{Q}$ is the scattering vector, $\overrightarrow{r_{n}}$ is the position vector of the nth atom in the system, and $\mathrm{N}$ is the number of atoms in the measured system. In Equation 10 the first term represents the coherent scattering and the second is for the incoherent scattering.

\subsubsection{Magnetic Neutron Scattering}

As mentioned earlier, neutrons can be scattered due to the interactions between their magnetic moment and the orbital and spin magnetic moments of the atoms 
in a crystal. The unpaired electron distribution is comparable to thermal neutron wavelength, and interference effects occur, which leads to a magnetic form factor. Figure 9 shows the neutron magnetic form factor compared to the x-ray form factor. The neutron magnetic form factor falls more rapidly compared to the X-ray form factor because only outer electrons are involved in the magnetic scattering [5]. The geometry of the magnetic neutron scattering is shown in Figure 14. The unit vectors

$\hat{\kappa}, \hat{\mu}$, and $\hat{P}$ are the scattering vector, the atom magnetic moment, and polarization vector respectively. The magnetic interaction vector $\vec{q}$ is defined as:

$$
\vec{q}=\hat{\kappa} \times(\hat{\mu} \times \hat{\kappa})=\hat{\mu}-(\hat{k} \cdot \hat{\mu}) \hat{\kappa} \quad \text { with }|q|=\sin \alpha
$$

where $\alpha$ is the angle between $\hat{\mu}$ and $\hat{\kappa}$. Bacon [6] described the the magnetic cross section for neutron magnetic scattering for atoms with parallel or anti-parallel magnetic moments as:

$$
\left(\frac{d \sigma}{d \Omega}\right)_{m a g .}=q^{2} S^{2}\left(\frac{e^{2} \gamma}{m_{e} c^{2}}\right)^{2}|f|^{2}
$$

where $S$ is the spin quantum number of the atom, $e$ is the charge of the electron, $m_{e}$ is the electron mass, $c$ is the speed of light, $\gamma$ is the magnetic moment of the neutron, and $f$ is the magnetic form factor.

When the magnetic moments are highly correlated as in ordered magnetic structures, the magnetic neutron scattering is coherent and gives strong diffraction effects.

\subsubsection{Neutron Beam Production}

The main neutron beam sources for research are either a nuclear reactor or a spallation source. Each of the two methods is different and thus yield neutron beams of neutron beams of different characteristics energies. At nuclear reactor sources, a self-sustaining critical fission nuclear reaction produces excess neutrons as a byproduct of the chain reaction. The coolant of the heart of the reactor acts as a reflector to concentrate neutrons. A moderator slows the neutrons. The resulting 


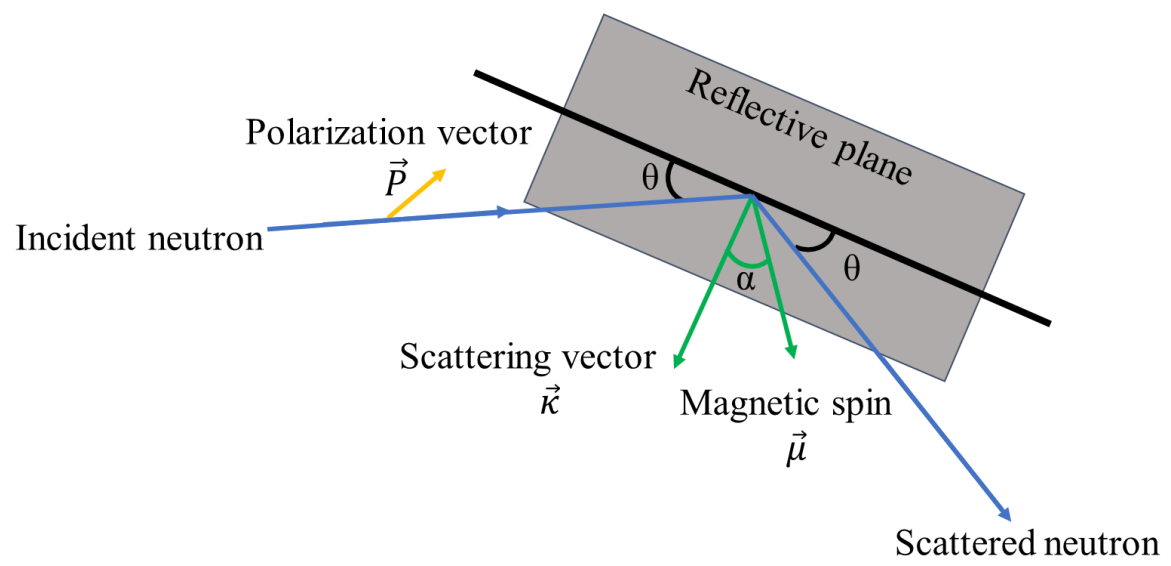

Figure 14. Geometry of the neutron magnetic scattering. The vectors $\hat{\kappa}, \hat{\mu}$, and $\hat{P}$ are the scattering vector, the atom magnetic moment, and polarization vector respectively. $\theta$ is the angle between incident or scattered neutron and the reflective plane. $\alpha$ is the angle between the scattering vector $\hat{\kappa}$ and the magnetization vector $\hat{\mu}$. The magnetic interaction vector $\vec{q}$ lies in the plane of $\hat{\kappa}$ and $\hat{\mu}$ and is perpendicular to $\hat{\kappa}$ (adapted from $[5,6]$ ).

neutrons are thermal neutrons with a Maxwellian distribution of wavelengths are in the range of $1-2 \AA$.

At a spallation neutron source (SNS), high-intensity neutrons are generated. The SNS ion source produces $\mathrm{H}^{-1}$ ions that are formed into a pulsed beam. This beam is delivered to a large linear accelerator (LINAC) where it accelerates to $1 \mathrm{GeV}$ which is equivalent to $90 \%$ the speed of light. The LINAC consists of normal conducting and superconducting radio-frequency cavities that accelerate the beam and a magnetic lattice that provides focusing and steering. LINAC cavities are cooled with liquid helium to a temperature of $2 \mathrm{~K}$. The $\mathrm{H}^{-1}$ ions pulse from the LINAC is wrapped into the ring through a diamond stripper foil that strips the electrons from the negatively charged hydrogen ions to produce the protons $\mathrm{H}^{-1}$ that circulate in the ring. Approximately a thousand turns are accumulated and then all these protons are "kicked" out at once producing a pulse less than 1 millionth of a second $\left(10^{-6}\right.$ seconds) in duration that is delivered to the target. At the target vessel which is a heavy metal 
(like $\mathrm{Pb}, \mathrm{W}$, Ta or $\mathrm{Hg}$ ). During neutron production, proton pulses collide with the target. When high-energy protons hit the nucleus of heavy metal atoms, neutrons are spalled or released. The neutrons emerging from the target are slowed down by moderators that are located above and below the target. Slowed neutrons, to low-energy neutrons suitable for research, are guided into beam tubes attached to instrument stations. The advantage of using a pulsed neutron source is that it provides highly effective intensity neutrons which are obtained for the same average neutron production. The pulse source defines one element of the resolution function. It has a lower background and nearly constant resolution. Spallation neutron sources are capable of providing peak thermal neutron fluxes at least an order of magnitude greater than those available from reactors. One disadvantage to the pulsed neutron beams is that time-of-flight (T.O.F.) techniques are obligatory [61].

\subsubsection{Neutron Beamlines Used in This Research}

\subsubsection{POWGEN}

POWGEN is a third-generation design of a time-of-flight (TOF) powder diffractometer at a spallation neutron source (SNS), ORNL. The instrument is optimized for both parametric studies of materials under a wide range of conditions like temperature, pressure, flowing gases, and ab-initio crystal structure determinations of complex solid-state materials. The geometric design of the instrument allows for all detected scattered neutrons to be focused onto a single diffraction profile yielding a high count rate while preserving good resolution data over $d$-spacings 0.3 - $10 \AA$ with

$\frac{\delta d}{d}=0.0015$ at a $d=1 \AA$. The diffractometer consists of three major components, the sample vessel, secondary collimation, and detectors. The high-resolution requirement dictates that POWGEN has a long $60 \mathrm{~m}$ flight path from moderator to sample and consequently must be housed in a separate building outside the main instrumental hall. In order to control the sample environment, POWGEN diffractometer started 
to use an orange cryostat with a temperature range from $2 \mathrm{~K}$ to $300 \mathrm{~K}$ and an IPNS Lucyfe furnace with a temperature range of $200-1000^{\circ} \mathrm{C}$ using a powder sample in vanadium can. In a time-of-flight powder diffractometer, the data is recorded as the number of neutrons as a function of time-of-flight. The de Broglie wavelength is modified to be as follows:

$$
\lambda=\frac{h t}{m_{n} L}
$$

Where $h$ is the Plack's constant, $t$ is the TOF, $m_{n}$ is the neutron mass, and $L$ is the total neutron flight path. The interatomic spacing $d_{h k l}$ is given by:

$$
d_{h k l}=\frac{h t}{2 m L \sin \theta}
$$

Where the units for $t$ is $\mu s, d$ is in $\AA$, and $L$ is in meters ( $\mathrm{L}=60 \mathrm{~m}$ for POWGEN) $[5,63]$.

\subsubsection{NOMAD}

The Nanoscale Ordered MAterials Diffractometer (NOMAD) is a neutron timeof-flight diffractometer designed to determine pair distribution functions (PDF). It can be used for a wide range of materials from short-range ordered liquids to longrange ordered crystals. The determination of the PDF by diffraction experiments involves the measuring of the scattered intensity as a function of momentum transfer

$\vec{Q}$. The momentum transfer is a Fourier transform of the pair distribution function (as discussed in the PDF section). High intensity, large accessible momentum transfer range, high stability, and good momentum transfer resolution are all requirements needed to get good results in PDF experiments. NOMAD, at the moment, is the fastest neutron diffractometer in its class with proportionate stability and precision. It is already a valuable tool for the determination of both disordered materials and crystalline structures [64]. 


\subsection{Powder Diffraction}

The rapid growth in scientific knowledge in the last few decades has led to the discovery and fabrication of new materials. These materials are highly desired in many fields and applications, such as medical equipment. The need for new material types continues in today's market. Presently, a noticeable effort of scientific research focuses on discovering, fabrication, and analyzing new materials with desired properties. Analyzing new fabricated materials is an essential step in understanding their properties. A critical part of the analyzing process is to determine how the material compounds are arranged. One of the well-understood techniques in determining material cell arrangement is powder diffraction. The powder diffraction method was developed independently in Germany by Debye and Scherrer in 1916 and the United States by Hull in 1917. Powder diffraction provides powerful means for the identification of unknown minerals, and it might be the most widely used technique for characterizing materials. It is a very effective method for providing information on unit cell dimensions and can be described as an analytical technique for phase identification of crystalline material. Generally, the samples are made into a powdery form, consisting of fine grains of single crystalline material to be studied. One of the significant advantages of using powder diffraction is that it provides explicit material determination with minimal sample preparation requirements. Since the pioneering papers of Hugo Rietveld (1967 and 1969), powder diffraction analysis has been improved, which made it perform well in many applications.

The determination of unknown solids is critical in studies of geology, environmental science, material science, engineering, and biology. Powder diffraction can be used to characterize crystalline materials, identify fine-grained minerals such as clays, determine unit cell dimensions, and measure the purity of a sample. With specialized techniques, powder diffraction can also determine crystal structures using Rietveld

refinement, determine of modal amounts of minerals (quantitative analysis), charac- 
terize thin film samples, and make textural measurements such as the orientation of grains in a polycrystalline sample.

To visualize diffraction from an ideal polycrystalline sample, we can use the Ewald construction, as shown in Figure $15 \mathrm{a}$ and b. In a powder sample, we have a large amount of randomly oriented scatters. The superposition of reciprocal lattice Ewald points from a powder sample with the same $d_{h k l}$ will form a cone, as shown in panel c from Figure 15. Since a crystal has many (hkl) planes ith d-spacing $d_{h k l}$, a powder diffraction pattern will consist of a set of concentric cones known as Debye-Sherrer cones. In a 2D detector, those cones will show as rings, which can be represented as a radial plot that represents the diffraction angle $2 \theta$ with the scattered intensity from the powder sample. The diffraction rings and the corresponding diffraction pattern are shown in Figure 16.

One essential limitation of the powder diffraction method is the loss of information due to the peak overlap. Modern advances in powder diffraction helped to overcome some of its limitations. Advances in powder diffraction technique over the last several decades are related to the development of analysis methods like the Rietveld method, structural solution methods, instrumentation, and new software packages. Also, newly developed algorithms to determine the structure of powder diffraction data, had improved significantly in recent years especially the quality of the intensity extraction. Software packages are capable of deconvoluting non-equivalent overlapping reflections accurately for reciprocal space structure solution. The usage of synchrotron x-ray has added advantages over lab x-ray data such as tunability, high brilliance, and relatively low divergence. Data from synchrotron sources can be collected on very small samples by using smaller wavelength x-ray, which reduces systematic errors associated with larger wavelengths. Development of pulsed neutron sources and the availability of time-of-flight neutron diffractometers has allowed collecting a full diffraction pattern with a constant resolution, which is another achievement 
a)

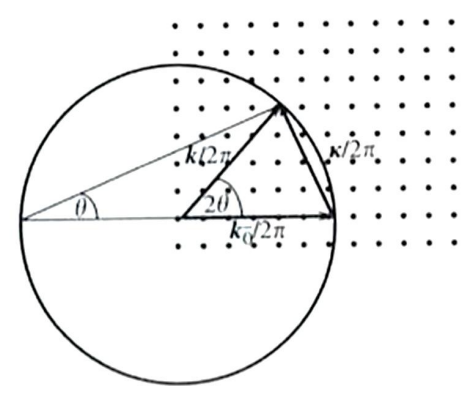

b)

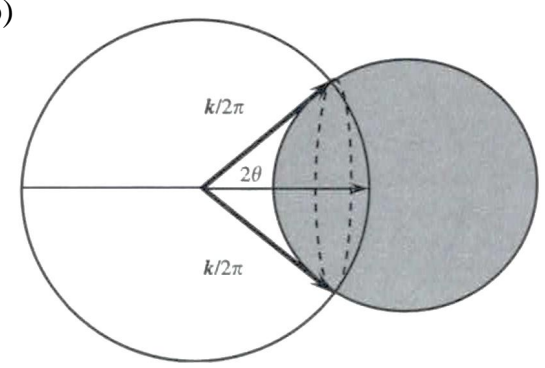

c)

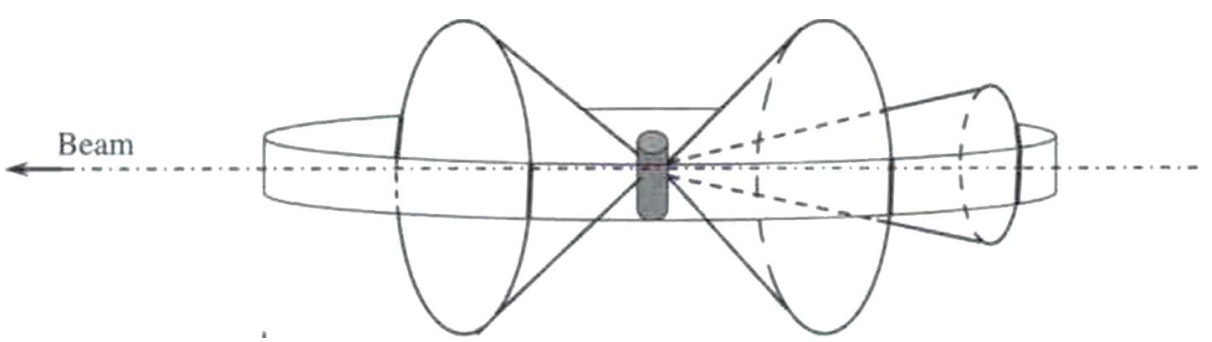

Figure 15. Panels a and $b$ show the Ewald construction. In panel a, the 2D section of the reciprocal lattice is shown, which represents a sphere with radius $1 / \lambda$. The incident, scattered wavevectors are $\overrightarrow{k_{0}} / 2 \pi$ and $\vec{k} / 2 \pi$. The scattering vector is $\vec{\kappa} / 2 \pi$. The diffraction condition is satisfied when the scattering vector ends at a reciprocal lattice point. Panel b shows the Ewald construction for powder diffraction were randomly oriented crystallites cause lattice points to be replaced by concentric spheres. The dashed line shows the cone of diffraction (Debye-Scherrer), which results as an intersection between the concentric sphere and the reflection sphere. Panel c shows an illustration of Debye-Scherrer concentric cones as a result of scattering from a powder sample. All illustrations are from reference [5]

for the powder diffraction method. The non-ambient sample environment for diffraction methods such as low/high temperature, high-pressure, and the ability to apply magnetic fields has significant advantages. 

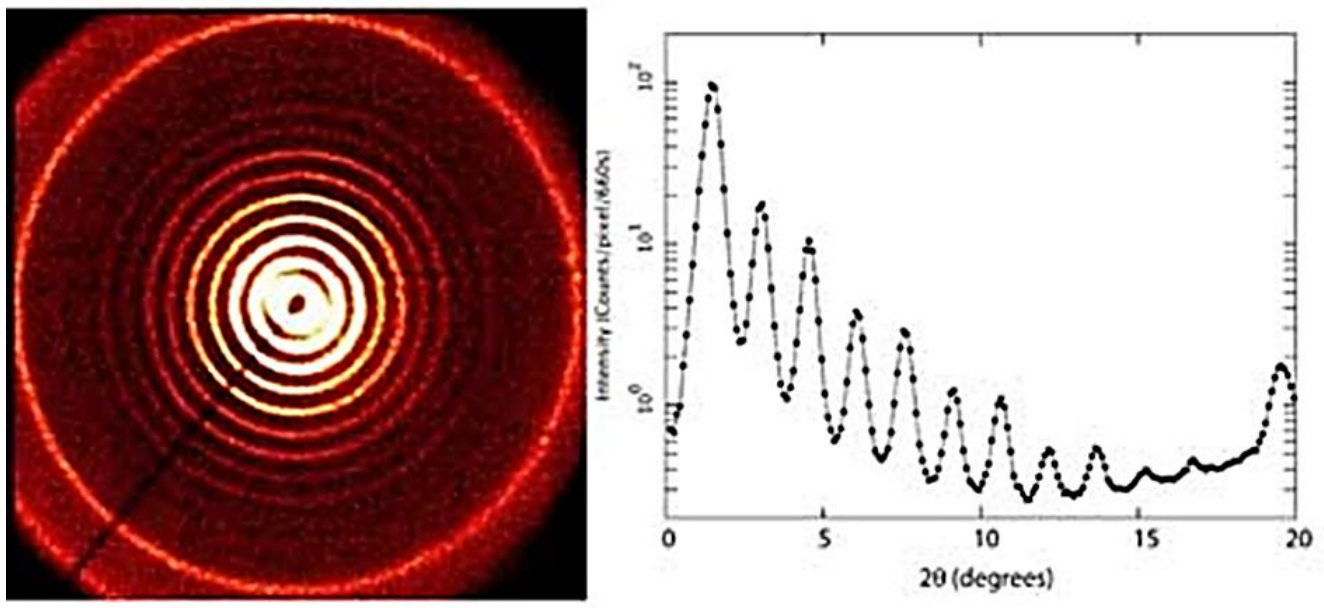

Figure 16. A powder diffraction ring pattern from silver behenate (a layered organic crystal) and the radial line plot of the scattered intensity (from https://www.physics.upenn.edu/ heiney/datasqueeze/basics.html). 


\section{CHAPTER 4}

\section{ANALYSIS METHODS}

\subsection{Rietveld Refinement}

The development of computers and the ability to use programmed codes in the 1960s enabled the use of the least-squares methods to refine structures. These methods were first applied to the refinement of single crystal structures and later used to the analysis of powder diffraction data. Hugo M. Rietveld received the Gregori Aminoff Prize in 1995 in recognition of his development of profile refinement methods for the analysis of powder diffraction data. His pioneer work in developing this method is demonstrated in the famous papers published in 1967 and 1969 on structure refinement $[65,66]$. Rietveld noticed that if a pattern could be modeled, the fit between a calculated pattern and measured data could be optimized. The Rietveld method, in the early 1980s, was developed to refine structural data collected with time-of-flight neutrons and synchrotron x-ray. This, along with the development of software packages for Rietveld refinement like DBWS and GSAS permitted a more precise and accurate data analysis from powder diffraction. Rietveld recognized that a diffraction pattern is a smooth curve that consists of Gaussian peaks on top of a smooth background and the best way of extracting the maximum information is to represent the observed intensity as:

$$
Y_{c}=Y_{b}+\sum Y_{h k l}
$$

where $Y_{c}$ is the calculated model of the diffraction, $Y_{b}$ is the contribution from the background, and $Y_{h k l}$ is the Bragg reflection [59]. The model function Equation (15) 
components are parametrized by the crystal parameters (atomic coordinates, thermal displacements parameters $U_{x x}$ or $U_{i s o}$, and site occupancies ) and by the diffraction experiment parameters (unit cell $(a, b$ and $c)$, peak profile broadening $(U, V, W$ or $X, Y)$, instrumental parameter $(\mathrm{Z}, \lambda)$, etc.). The adjustable parameters are refined by the least-squares method in order to minimize the weighted difference between the observed and the calculated intensities. A minimization of:

$$
M=\sum w\left(Y_{o}-Y_{c}\right)^{2}
$$

will give an estimate of the minimum variance. The weight $w$ is computed from the variance of $Y_{o}$. The quality of the least-squares refinement is shown by the residual function $R_{p}$, the weighted residual function $R_{w p}$, the goodness-of-fit GoF, and the reduced $\chi^{2}$ which are defined as:

$$
\begin{gathered}
R_{p}=\frac{\sum\left|Y_{o}-Y_{c}\right|}{\sum Y_{o}} \\
R_{w p}=\frac{M}{\sum w Y_{o}^{2}} \\
G o F=\sqrt{\frac{\sum M}{N_{o b s}-N_{v a r}}} \\
\chi^{2}=\frac{\sum M}{N_{o b s}-N_{v a r}}
\end{gathered}
$$

where $N_{o b s}$ is number of observations and $N_{v a r}$ is the number of variables during the refinement process. The

\subsection{Pair Distribution Function}

The Pair Distribution Function (PDF) method is a total scattering technique for determining the local order in nanostructured materials [67]. PDF was first published in 1915 soon after the first x-ray diffraction experiments were performed [67]. In 
1927, Zernicke and Prins derived the Fourier relationship between the real-space pair density and the Debye scattering equation, which lead to the creation of the PDF method. The very first x-ray PDF experiments were carried out by Debye himself with Menke in 1930. [68]. The Debye equation was based on the average intensity distribution as:

$$
I(Q)=\sum \sum f_{i}(Q) f_{j}^{*}(Q) \frac{\sin \left(Q r_{i j}\right)}{Q r_{i j}}
$$

where $Q=4 \pi \sin (\theta) / \lambda$, where $\lambda$ is the scattering wavelength and $2 \theta$ is the scattering angle, $f$ is the atomic form factor, and $r_{i j}$ is the magnitude of the distance between any two of the $N$ atoms in the sample [69].

The PDF is a measured probability of finding an atom at a given distance $r$ from another atom. Therefore, the PDF method provides structural information about materials on different length scales. The PDF $g(r)$ is calculated by Fourier transforming the total scattering data which include Bragg peaks and the diffused scattering data. The reduced pair distribution function $G(r)$ is widely used and is related to $g(r)$ by $G(r)=4 \pi r[g(r)-1]$. The advantage of using $G(r)$ over $g(r)$ is that it is calculated directly from Fourier transform (equation 22).

$$
G(r)=2 \pi \int_{Q_{\min }}^{Q_{\max }} Q[S(Q)-1] \sin (Q r) d Q
$$

The structure function, $S(Q)$, is extracted from the Bragg and diffuse components of x-ray, neutron, or electron powder diffraction intensity.

$$
G_{c}(r)=\frac{1}{r} \sum_{i} \sum_{j}\left[\left(\frac{b_{i} b_{j}}{<b>^{2}}\right) \delta\left(r-r_{i j}\right)\right]-4 \pi r \rho_{0}
$$

The diffuse scattering offers important information about the local deviations from the average structure [67]. The calculated PDF, denoted $G_{c}(r)$, is calculated by equation 23 from the structural model [70]. Here, $\rho_{0}$ is the atomic number density of the material and $\rho(r)$ is the atomic pair density, which is the mean weighted density of neighbor atoms at distance $\mathrm{r}$ from an atom at the origin. The sum $\rho(r)$ is over all 


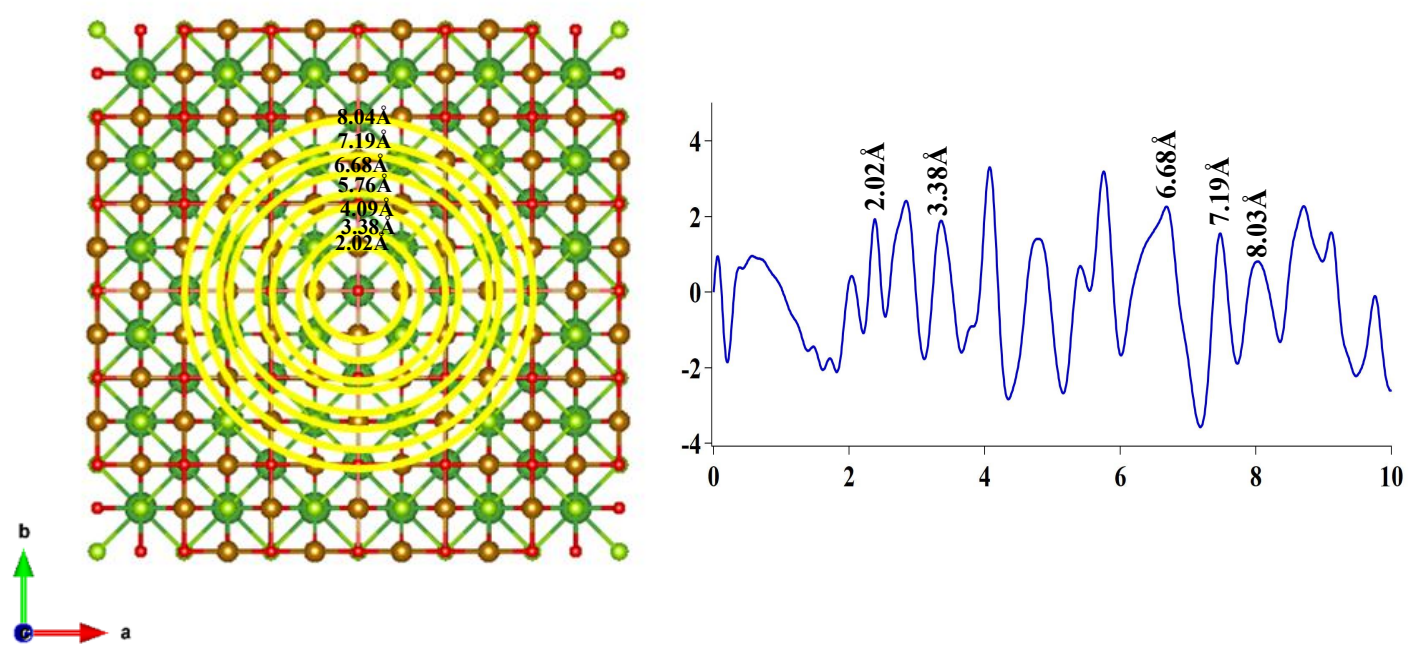

Figure 17. PDF method measure the inter-atomic distances between atoms in the crystal. Each peak corresponds to a distance, where neighbouring atoms are most likely found in the sample.

the atoms in the sample, $b_{i}$ is the scattering factor of atom $i,\langle b\rangle$ is the average scattering factor and $r_{i j}$ is the distance between atoms $i$ and $j$ [68]. Figure 17 shows the PDF method and how the peaks in the total scattering data correspond to the inter-atomic distance of a sample.

PDF utilizes all diffraction information, including high $Q$ values and diffused scattering data. This is important, especially in cases where disorder exists in a crystalline system [67]. In practice, values of $Q_{\min }$ and $Q_{\max }$ are determined by the experimental set-up, and $Q_{\max }$ is often held below the experimental maximum to eliminate noisy data from the PDF since the signal to noise ratio becomes unfavorable in the high $Q$ region. Once the experimental PDFs are determined, they can be analyzed directly or modeled. PDF modeling, is performed by adjusting the parameters of the structure model, such as the lattice constants, atom positions, and anisotropic atomic displacement parameters in order to maximize the agreement between the calculated and an 
experimental PDF. The goodness-of-fit parameter, which measures the agreement, $\chi^{2}$ is calculated using equation 24 . Where the sum is all measured data $r_{i}$ and $G_{e}$ is the experimental reduced pair distribution function $G(r), G_{c}$ is the calculated reduced pair distribution function, and $\sigma$ is the estimated standard deviation. This procedure is implemented in PDFgui [71] and Diffpy-CMI [72], which are widely used for PDF analysis.

$$
\chi^{2}=\sum_{i=i}^{N} \frac{\left(G_{e}\left(r_{i}\right)-G_{c}\left(r_{i}\right)\right)^{2}}{\sigma^{2}}
$$

A powerful approach is simply to compare experimentally determined PDFs from samples under study and from known control samples. Insightful information can be learned simply from visual inspections and by taking differences to look for residual signals. Numerical tools that compare the likeness, or degree of correlation, between two curves also give insight.

The use of scattering techniques is very common in condensed matter physics. Scattering techniques provide unique results about the nuclear and magnetic structure of a material and its behavior. It is difficult to obtain similar information by using other techniques such as Raman scattering and NMR. For this reason we used neutron and x-ray scattering to understand the properties of the materials investigated in this work. For studying the nuclear and magnetic properties of Mott-insulators like $\mathrm{Ca}_{2} \mathrm{O}_{2} \mathrm{Fe}_{2.6} \mathrm{OS}_{2-x} \mathrm{Se}_{x}$ and the WSM MoTe 2 , powder neutron scattering was performed at POWGEN (ORNL) the Canadia Neutron Beam Center. X-ray powder diffraction at 11-BM (APS) was performed to complement the neutron data. Rietveld refinement was used to analyze both neutron and x-ray powder diffraction data. In order to examine local structure of $\mathrm{La}_{2} \mathrm{O}_{2} \mathrm{Fe}_{2} \mathrm{O}(\mathrm{S}, \mathrm{Se})_{2}$, neutron total scattering experiments was performed at NOMAD, ORNL. PDF analysis was used to analyze this experimental data. 


\subsection{Electrical Resistivity}

The relation between the temperature and the resistivity of a material can be shown as $\rho(T)=\rho_{o}\left[1+\alpha\left(T-T_{o}\right)\right]$, where $\rho_{o}$ is the resistivity at a standard $T_{o}$, and ais coefficient of resistivity. For metals, the coefficient of resistivity, $\alpha$, is positive, and for semiconductor and insulators, it is negative.

In doped semiconductors, the Arrhenius temperature dependence resistivity follows equation 25 .

$$
\rho(T)=\rho_{o} \exp \left(\frac{E_{a}}{k_{B} T}\right)
$$

where $\rho_{o}$ is the pre-exponential factor, $\mathrm{E}_{a}$ is the activation energy and $K_{B}$ Boltzmann constant. Many transition metal compounds display resistivity saturation at high temperatures, where the resistivity slope decreases significantly as a function of temperature [73].The resistivity usually level close to Mott-Ioffe-Regel (MIR) limit of $\rho_{M I R}=3 \pi^{2} \hbar / e^{2} k_{f}^{2} a$, where $a$ is the lattice parameter, is $\approx 100-3000 \mu \Omega \mathrm{cm}[74,73]$.

In Mott insulators, the metal-to-insulator (MIT) transition can be driven by different kind of external perturbations, such as thermal energy, electric field, and optical stimuli $[7]$. In the initial state the material in in the insulating phase when a perturbation is introduced a reduction in the resistivity several order of magnitude. If the perturbation is removed the system return to its original insulating state (Figure 18).

\subsubsection{Variable-Range Hopping}

In Mott insulator, where the ground state is still insulating, a strong correlation between bad-metal state and Mott variable-range hopping model VRH localized state

exists where the size of the Mott VRH activation energy $\mathrm{E}_{a}$ is linearly proportional to the critical temperature $T_{c}$ [74]. The Mott VRH describes the hopping conduction at low temperatures in strongly disordered systems with localized charge-carrier states 


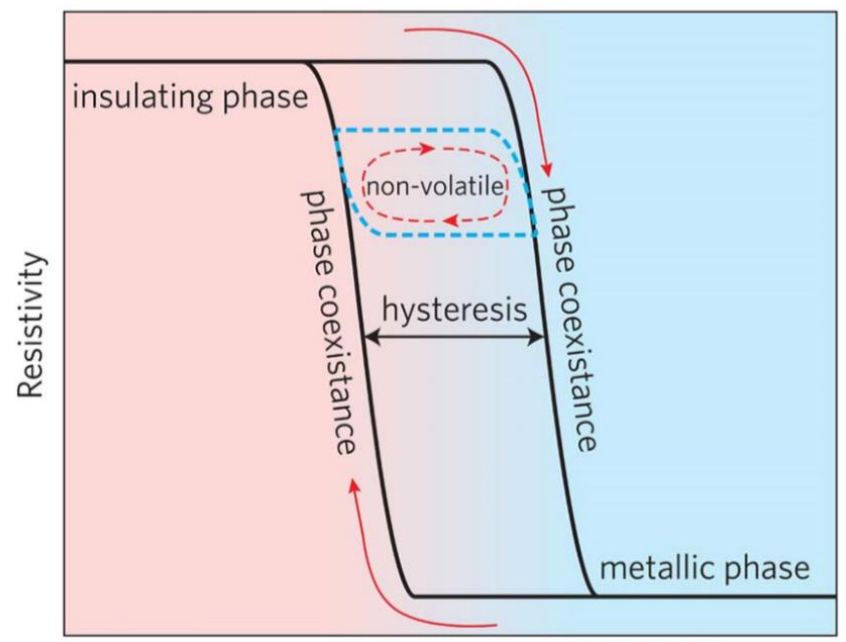

External excitation

(thermal, electrical, optical, mechanical and other)

Figure 18. Metal-insulator-transition features the figure from reference [7].In Mott insulators, the metal-to-insulator (MIT) transition can be driven by different kind of external perturbations.

[75] as in equation 26:

$$
\rho=\rho_{o} \exp \left(T_{o} / T\right)^{1 / 4}
$$

where $\mathrm{A}$ is a pre-factor constant, $T_{o}$ is a characteristic temperature [75]. This can be generalized to Equation 27:

$$
\rho(T)=\operatorname{Aexp}\left(\frac{T_{o}}{T}\right)^{p}
$$

where $\mathrm{A}$ is a pre-factor constant, $T_{o}$ is a characteristic temperature, and $p$ denotes the value of the exponent reflecting the hopping dimensionality and Coulomb interactions [76]. The value for $p$ represent the dimentionality of the hopping mechanism and is equal to $\frac{1}{(d+1)}$ where $\mathrm{d}=1,2$, and 3 . A linear curve is expected when the $\operatorname{Ln}(\rho)$ vs. $T^{1 / p}$ and the Mott activation energy is calculated from its slope. 


\subsection{Magnetic Properties}

In the presence of a magnetic field, the thermodynamic identity can be written as:

$$
d F=-S d T-M d B
$$

Where $F$ is the free energy, $B$ is the external magnetic field and $M$ is the total magnetic moment of the system. Then the magnetization can be expresses as :

$$
M=\left(\frac{\partial F}{\partial B}\right)_{T}
$$

The energy of the magnetic dipole in the presence of a magnetic field is $E=-\gamma S B$ where $S$ is the spin with values \pm 1 and $\gamma$ is the magnitude of the magnetic moment. The probability of a state having energy E is:

$$
P_{\uparrow \downarrow}=\frac{e^{ \pm \beta \gamma B}}{e^{\beta \gamma B}+e^{\beta \gamma B}}
$$

Then the expectation of the magnetic moment $m$ is:

$$
m=\gamma P=\gamma \tanh \left(\frac{\gamma B}{k_{B} T}\right)
$$

For small magnetic fields where $\frac{\gamma B}{k_{B} T}<<1$ then :

$$
m=\frac{\gamma^{2}}{k_{B} T} B
$$

So $m \propto B$ and the constant of proportionality $\frac{\gamma^{2}}{k_{B} T}$ is called the magnetic susceptibility $\chi:$

$$
\chi=\frac{C}{T}
$$

Equation 33 is called Curie's law and the constant $C=\frac{\gamma^{2}}{k_{B}}$. The Curie temperature $T_{c}$ is the temperature above which the spontaneous magnetization vanishes; it separates the disordered paramagnetic phase at $T>T_{c}$ from the ordered ferromagnetic phase at $T<T_{c}$. In paramagnetic material, the magnetic susceptibility obeys the Curie law. In situations where there are short-range ferromagnetic or antiferromagnetic 
domains, the magnetic susceptibility $\chi=\frac{C}{T-\theta}$ is called Curie-Weiss law, and $\theta$ is the Curie-Weiss temperature. In an antiferromagnet material, the spins are ordered in an antiparallel arrangement with zero net moments at temperatures below the magnetic ordering temperature or the Neél temperature. The susceptibility at $T=T_{N}$ of an antiferromagnet is not infinite but has a discontinuity. The temperature dependent magnetic susceptibility in paramagnet (Figure 19 a), ferromagnets (Figure 19 b), and antiferromagnets (Figure $19 \mathrm{c}$ ), where the magnetic transition temperature $T_{N} \mathrm{~b}$ shows as a kink in the magnetic susceptibility and has a maximum value.
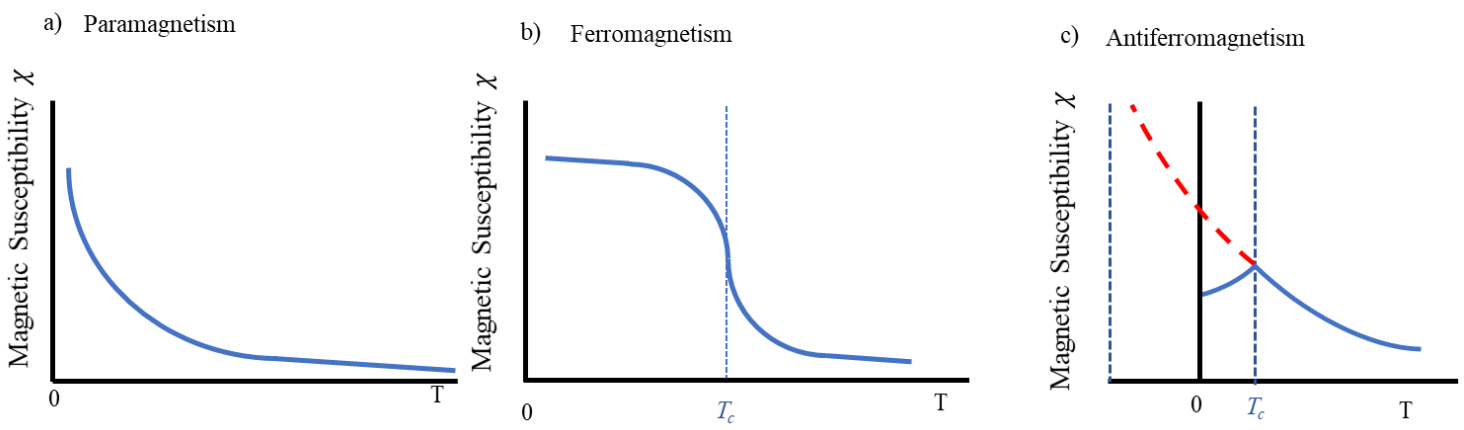

Figure 19. Temperature dependent magnetic susceptibility behavior in different materials adapted from [8]

In iron oxychalcogenides, the temperature-dependent magnetic susceptibility has a very distinct feature, and it usually shows a broadening peak at the Neél temperature $T_{N}$. The sharp discontinuity shown in Figure $20 \mathrm{c}$ is broadened. This indicates the onset of the long-range antiferromagnetic ordering for $T<T_{N}$ (Figure 20) $[9,10$, 11, 77]. The broadening of magnetic susceptibility maximum and its deviation from the magnetic Curie-Weiss law, in the paramagnetic phase above $T_{N}$, indicates strong antiferromagnetic short-range correlations [77, 10]. 

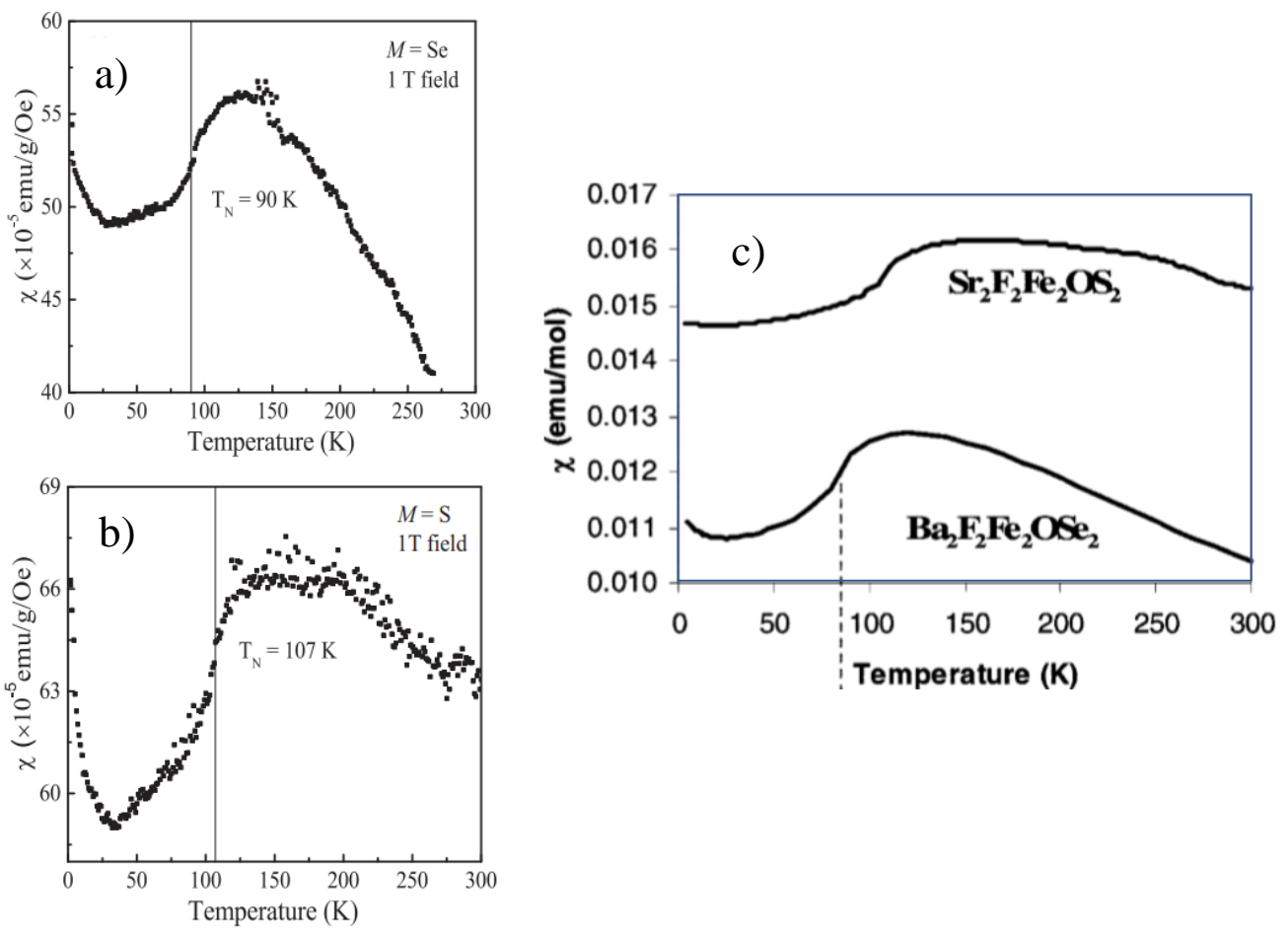

Figure 20. Temperature dependant magnetic susceptibility in iron oxychalcogenides Mott insulators showing the broad maximum that characterize magnetic susceptibility in this material. Panels a and b show magnetic susceptibility in $\mathrm{La}_{2} \mathrm{O}_{2} \mathrm{Fe}_{2} \mathrm{OSe}_{2}$ and $\mathrm{La}_{2} \mathrm{O}_{2} \mathrm{Fe}_{2} \mathrm{OS}_{2}$ respectively, where a Curie tails are observed at low temperature from [9]. Panel c) shows a typical magnetic susceptibility in iron oxychalcogenides as in $\mathrm{Sr}_{2} \mathrm{~F}_{2} \mathrm{Fe}_{2} \mathrm{OS}_{2}$ and $\mathrm{Ba}_{2} \mathrm{~F}_{2} \mathrm{Fe}_{2} \mathrm{OS}_{2}$ from [10] 


\section{CHAPTER 5 \\ STRUCTURAL STUDY OF CALCIUM IRON OXYCHALCOGINIDES}

\subsection{Introduction}

The study of superconductivity high- $T_{c}$ (HTSC) in iron pnictides and chalcogenides, like that of HTSC Cu-based superconductors, is largely focused on understanding the relevance of electron correlation to the onset of the superconducting phase. Iron-based materials and cuprates enter the superconducting phase from different conditions. Copper oxide parent material are Mott insulators with strong electron correlation. On the other hand, the parent compounds of Fe-based superconductors are generally designated as Hund's metals that may exhibit metallic, semiconducting, or semimetallic behaviour in the normal state. In FeSC, the electron correlation can be related to Hund's rule coupling whereas electron correlation in cuprates is thought to be an effective Hubbard interaction [78]. Numerous published works suggest that Fe-based superconductors are close to the Mott boundary; however, relatively few iron-containing materials, that show both Mott insulating behavior and superconductivity, have been discussed in the literature. The search for such materials has motivated further research of the iron oxychalcogenides $R E_{2} \mathrm{O}_{2} \mathrm{Fe}_{2} \mathrm{OM}_{2}(R E=$ rareearth atoms, $M=\mathrm{S}$, Se). These are paramagnetic (PM) Mott insulators in the normal phase and it has been argued that they can be driven toward the metallic phase, and possibly superconductivity, by doping $[79,13]$. However, superconductivity has not yet been reported in chemically doped variants of $R E_{2} \mathrm{O}_{2} \mathrm{Fe}_{2} \mathrm{OM}_{2}$ materials. 

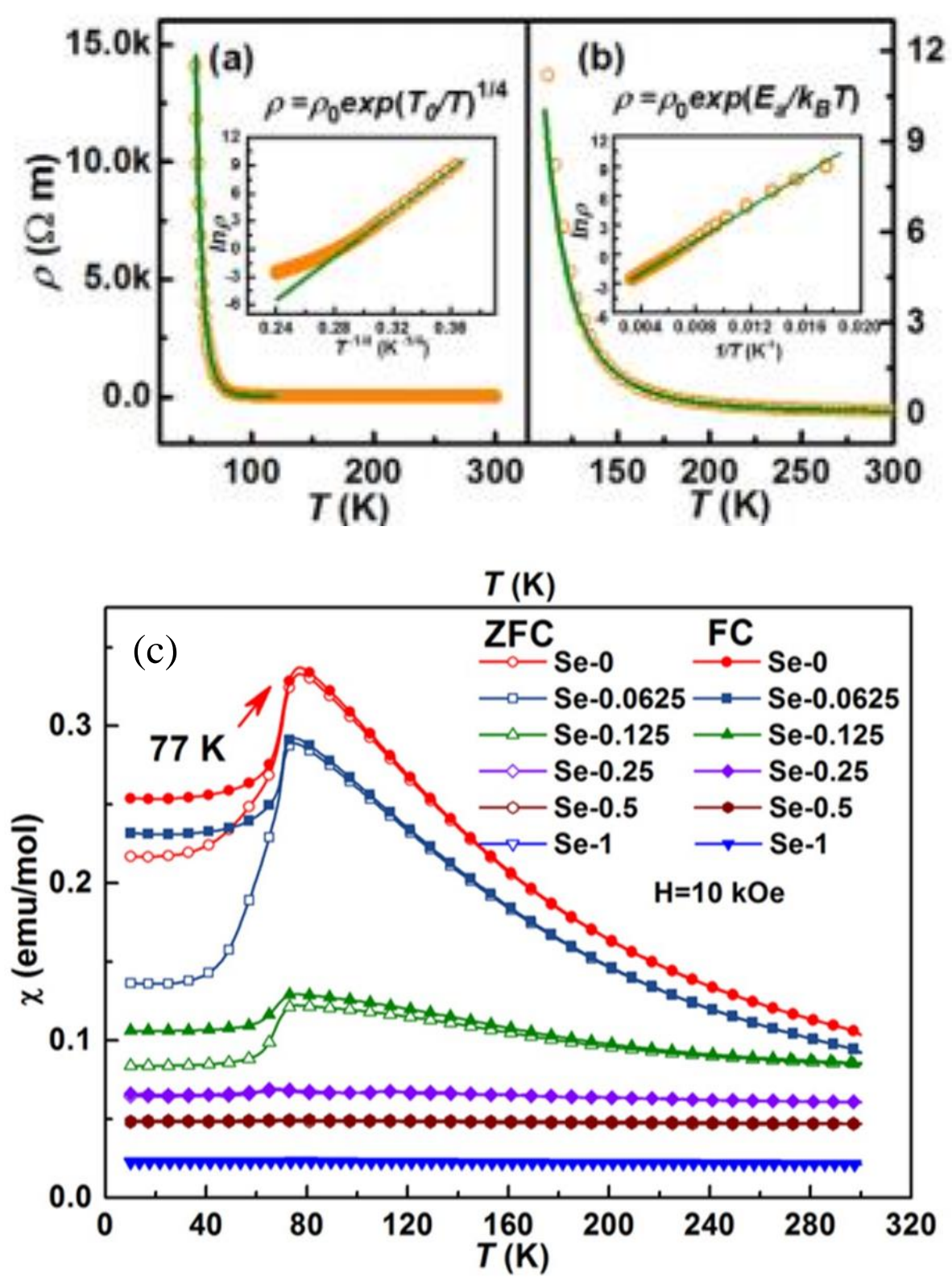

Figure 21. $\mathrm{Ca}_{2} \mathrm{O}_{2} \mathrm{Fe}_{2.6} \mathrm{OS}_{2}$ resistivity and magnetic susceptibility from reference [11].

Transport measurements indicate that $\mathrm{Ca}_{2} \mathrm{O}_{2} \mathrm{Fe}_{2.6} \mathrm{OS}_{2}$ is an $\mathrm{AFM}$ semiconductor with a strong electron correlation and narrow band gaps (see Figure 21) [11]. However, this electron correlation is unexpected because the Fe-Fe distance is the smallest among all the oxychalcogenides [11]. Band theory suggests that $\mathrm{Ca}_{2} \mathrm{O}_{2} \mathrm{Fe}_{2.6} \mathrm{OS}_{2}$ should be more metallic than other iron-oxychalcogenides (which are insulators) because of the short ion-ion distance ought to yield greater Fe band overlaps [8, 80]. Further- 
more, theoretical calculations, $\mathrm{LDA}+\mathrm{U}[11]$ and $\mathrm{LDA}+\mathrm{DMFT}[81]$, suggested that the stoichiometric variant $\mathrm{Ca}_{2} \mathrm{O}_{3} \mathrm{Fe}_{3} \mathrm{~S}_{2}$ should be metallic but this compound has yet to be successfully grown [11]. This is surprising since the smaller Fe-Fe bond length might be thought to induce metallicity due to the greater iron band overlap. The Fe-Fe bond lengths in $\mathrm{Ca}_{2} \mathrm{O}_{2} \mathrm{Fe}_{2.6} \mathrm{OS}_{2}$ are less than those in iron-pnictides, iron-chalcogenides as well as other iron oxychalcogenides.

Current theories of high temperature superconductivity suggest that electrons must organize into Cooper pairs in order for a material to exhibit superconducting behavior $[82,83]$. Superconductivity often emerges from insulators that are altered by doping or external stimuli. Electrons in insulators experience significant repulsive interactions that tend to keep electrons localized at atomic positions. In contrast, electrons in metals are delocalized, interact weakly, and are free to conduct electricity. Therefore, the formation of Cooper pairs should have different mechanisms for metals compared to insulators. This contrast is central to the debate about the origin of high temperature superconductivity in iron based materials. Many iron-based materials are metallic in the normal phase; however, before entering superconducting phase these systems are believed to harbor insulating characteristics in close proximity to a Mott insulating phase. Figure 22 shows the location of $\mathrm{Ca}_{2} \mathrm{O}_{2} \mathrm{Fe}_{2.6} \mathrm{OS}_{2}$ on iron pnictide phase diagram.

The newly reported iron oxychalcogenide [11] $\mathrm{Ca}_{2} \mathrm{O}_{2} \mathrm{Fe}_{2.6} \mathrm{OS}_{2}$ has space group $P 4 / m m m$ and consists of alternate stacking of antiperovskite like $\left[\mathrm{Ca}_{2} \mathrm{FeO} 2\right]^{2+}$ layers and $\left[\mathrm{Fe}_{2} \mathrm{OS}_{2}\right]^{2-}$ building blocks along the $c$ axis as illustrated in Figure 23. Figure 23 (right) shows the $\mathrm{Fe}_{2} \mathrm{OS}_{2}$ plan where the $\mathrm{Fe}^{2+}$ ions are located between oxygen atoms forming a square-planar layer. This is an anti-configuration with respect to the $\mathrm{CuO}_{2}$ layer of high- $T_{c}$ cuprates. The two-dimensional $\left[\mathrm{Fe}_{2} \mathrm{OS}_{2}\right]^{2+}$ layers contain $\mathrm{Fe}_{2} \mathrm{OS}_{2}$ planar sheets separated from the $\left[\mathrm{Fe}_{2} \mathrm{OS}_{2}\right]^{2-}$ layers by $\mathrm{Ca}$ ions. The $\mathrm{Fe} 2$ ions in the $\left[\mathrm{Fe}_{2} \mathrm{OS}_{2}\right]^{2-}$ blocks are six fold-coordinated by two $\mathrm{O}$ ions and four sulfur ions, forming 


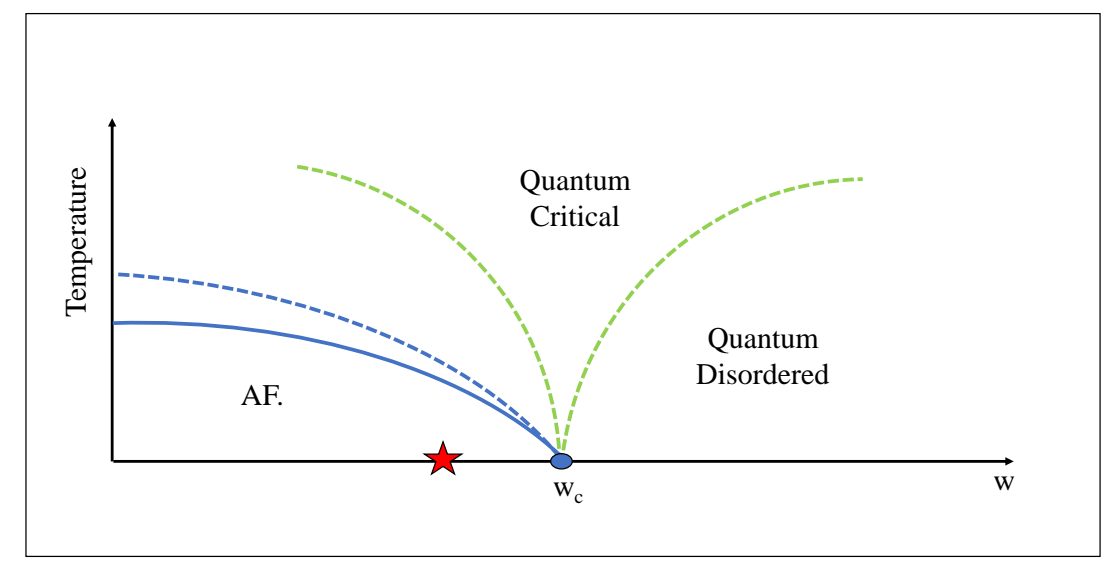

Figure 22. Pnictide phase diagram near a magnetic quantum critical point. The blue dot denotes the quantum critical point determined by the critical tuning parameter $\mathrm{w}_{c}$. The blue line is the line of the thermally driven antiferromagnetic transition and the dashed line is a structural transition [12]. The red star represents the location of $\mathrm{Ca}_{2} \mathrm{O}_{2} \mathrm{Fe}_{2.6} \mathrm{OS}_{2}$ within the phase diagram.

an $\mathrm{FeO}_{2} \mathrm{~S}_{4}$ octahedron. The $\mathrm{Fe}(1)$ sites are fully occupied, while the Fe2 ions sites are about $20 \%$ deficient [11].

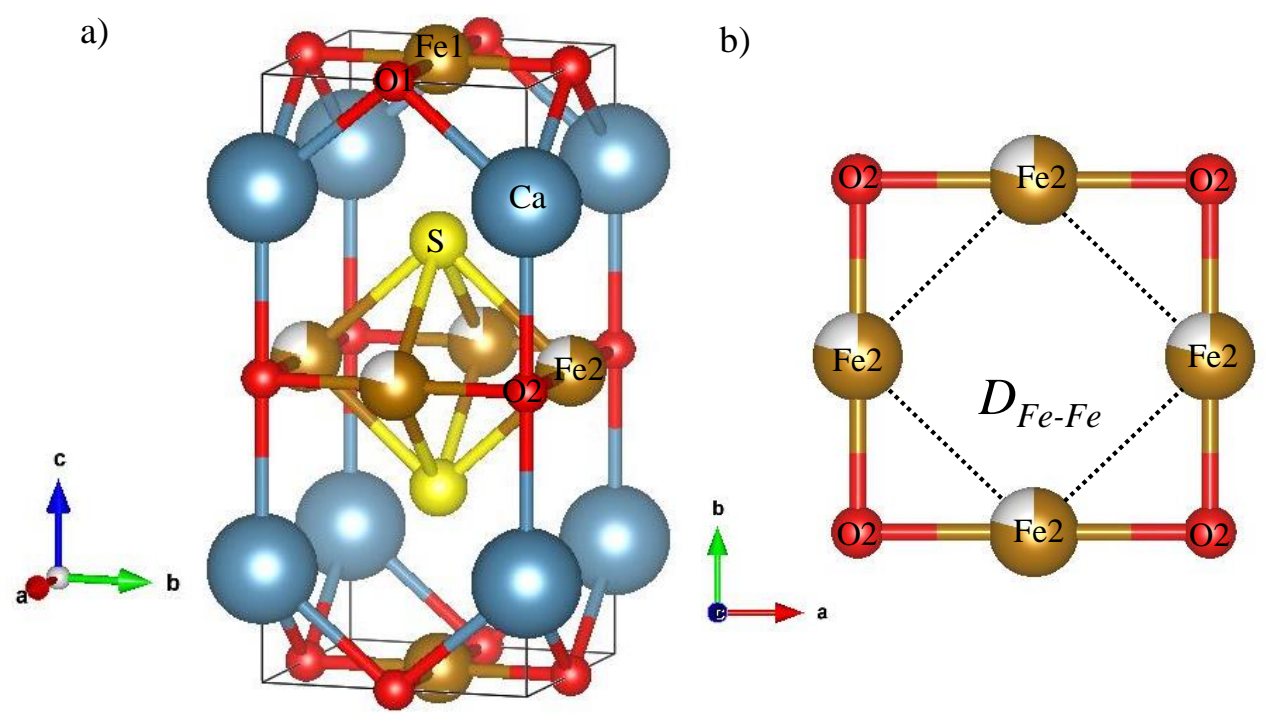

Figure 23. The crystal structure of $\mathrm{Ca}_{2} \mathrm{O}_{2} \mathrm{Fe}_{2.6} \mathrm{OS}_{2}$ is shown in panel a). Panel b) shows the $\mathrm{Fe} 2$ and $\mathrm{O} 2$ atoms forming the square-planer in the $\mathrm{Fe}_{2} \mathrm{OS}_{2}$ layer. The Fe-Fe distance $\left(D_{F e-F e}\right)$ between any two iron atoms in this plane is $2.7902 \AA$. 


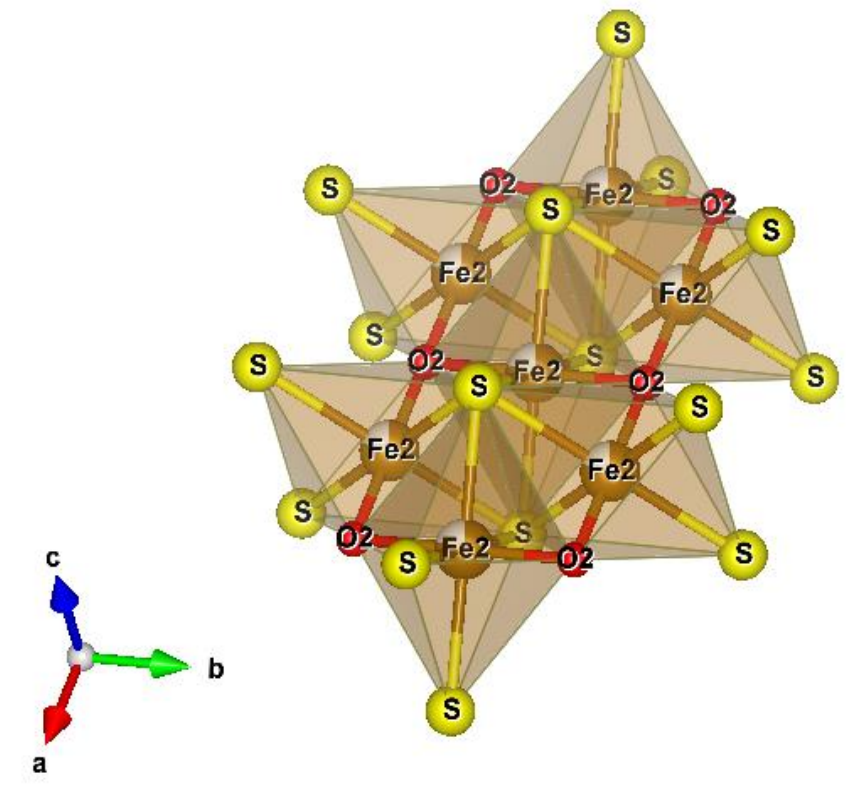

Figure 24. The octahedra in $\mathrm{Ca}_{2} \mathrm{O}_{2} \mathrm{Fe}_{2.6} \mathrm{OS}_{2}$ structure. Each octahedron consists of an $\mathrm{Fe} 2$ ion, two $\mathrm{O} 2$ ions, and four $\mathrm{S}$ ions forming $\mathrm{FeO}_{2} \mathrm{~S}_{4}$.

The decreasing of the Fe-Fe bond length $\left(d_{F e-F e}\right)$ in $\mathrm{Ca}_{2} \mathrm{O}_{2} \mathrm{Fe}_{2.6} \mathrm{OS}_{2}$ results in enhanced insulating properties instead of an expected increased metallicity [11, 77, 10]. This surprising behaviour illustrates the need for further study of calcium iron oxychalcogenide $\mathrm{Ca}_{2} \mathrm{O}_{2} \mathrm{Fe}_{2.6} \mathrm{OS}_{2}$ as shown in Figure 25. For this reason, we investigated the structural and magnetic ordering implications of this effect. We also performed the first investigation of the structural behavior of chalcogen-doped variant of $\mathrm{Ca}_{2} \mathrm{O}_{2} \mathrm{Fe}_{2.6} \mathrm{OS}_{2}$. We concentrated our efforts to better understand the nuclear structural therefor, the magnetic Rietveld refinement was not done in this work even though we collected magnetic data from neutron powder diffraction experiment. The presented work shows results of the structural Rietveld analysis of data obtained from neutron diffraction experiments conducted at POWGEN beamline at the ORNL, high-resolution XRD from 11-BM, APS and lab-based XRD. 


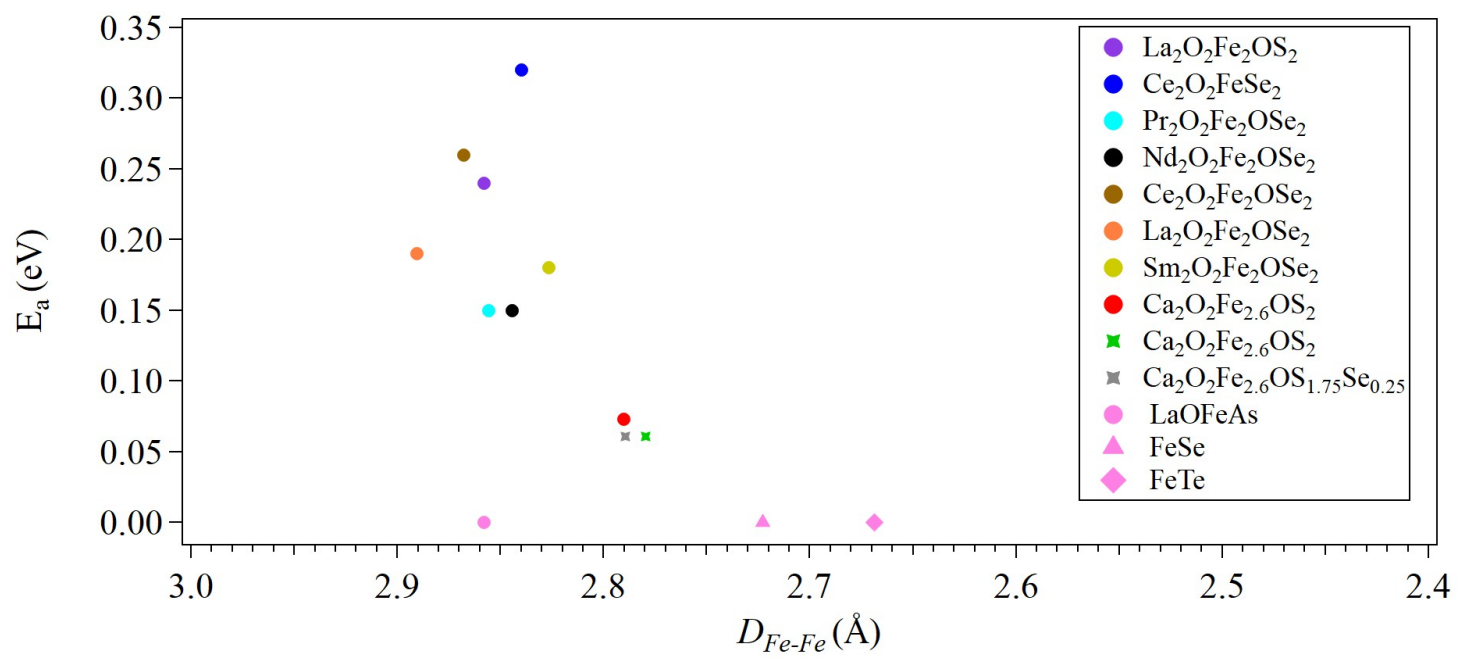

Figure 25. Activation energy dependence of the Fe-Fe bond length $\left(D_{F e-F e}\right)$ iron oxychalcogenides including the materiel in this study which are shown in gray and green stars. Pink markers shows iron based superconductors. Activation energy and bond length values used in this graph were from references [11, 13, 9, 14, 15]

\section{$5.2 \quad$ Experimental Methods}

\subsubsection{Synthesis}

$\mathrm{Ca}_{2} \mathrm{O}_{2} \mathrm{Fe}_{2.6} \mathrm{OS}_{2-x} \mathrm{Se}_{x}(x=0$ and 0.25$)$ samples were fabricated at the Beijing National Laboratory for Condensed Matter Physics by Professor Minghu Fang's research group. The appearance of synthesized samples were shiny, black single crystals or polycrystalline materials. Powder samples were made by grinding reagents with an agate pestle and mortar and then pressing the mixture into pellets under glove box conditions. Pellets were placed in an alumina crucible and sealed inside an evacuated quartz tube. The sealed quartz tube was heated slowly to $1173 \mathrm{~K}$ and held at this reaction temperature for 24 hours before furnace cooling to room temperature. The resulting single crystalline material has a black plate-like form with typical sizes of $0.5 \times 0.5 \times 0.5 \mathrm{~mm}^{3}$. Efforts to produce large single-crystal of $\mathrm{Ca}_{2} \mathrm{O}_{2} \mathrm{Fe}_{2.6} \mathrm{OS}_{2-x} \mathrm{Se}_{x}$ have proven to be unsuccessful and, to the best of our knowledge, there are no published reports of large single crystals. 


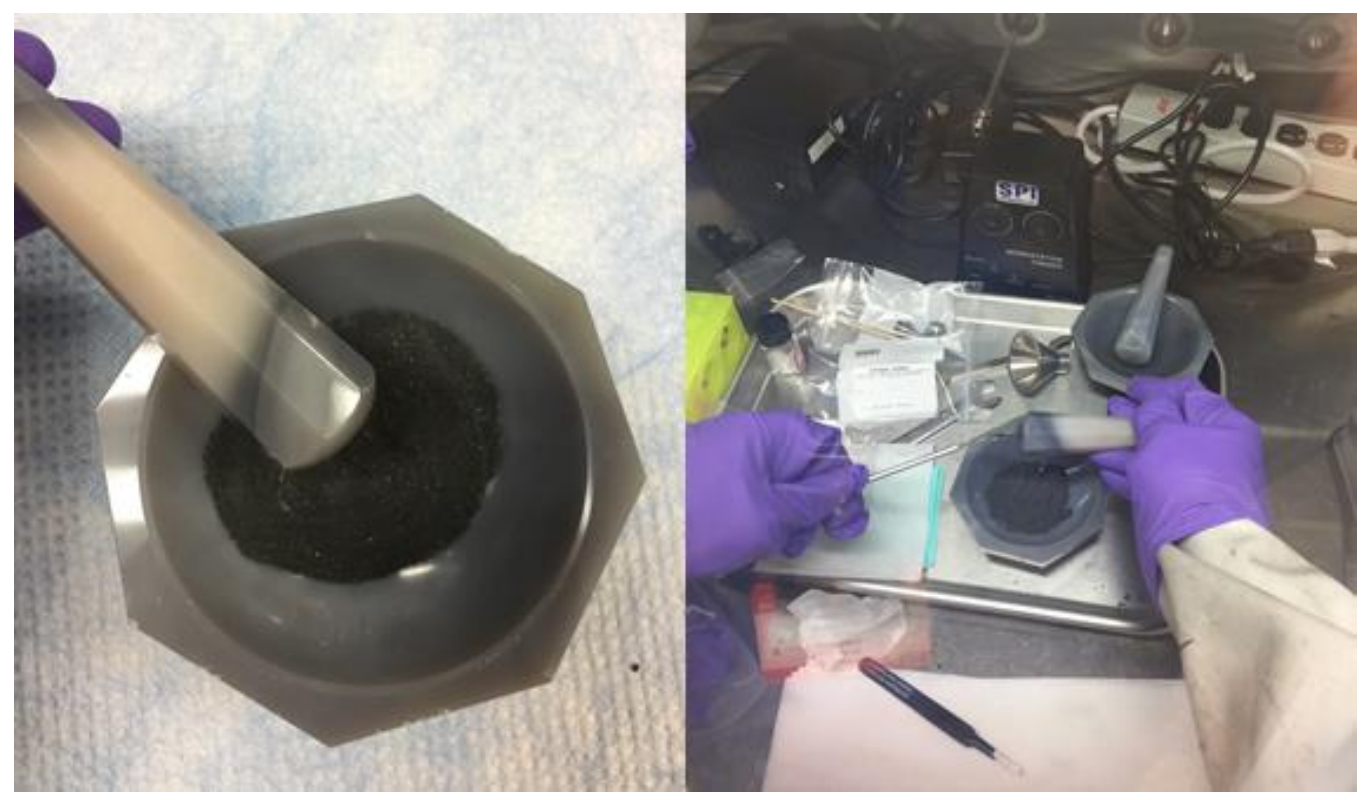

Figure 26. Powdering a sample with mortar and pestle as part of sample prepration for POWGEN experiment.

\subsubsection{Diffraction Experiments}

\subsubsection{X-ray diffraction}

High-resolution x-ray powder diffraction experiments were performed on two materials through the mail-in program at 11-BM, APS. The samples $\mathrm{Ca}_{2} \mathrm{O}_{2} \mathrm{Fe}_{2.6} \mathrm{OS}_{2}$ and $\mathrm{Ca}_{2} \mathrm{O}_{2} \mathrm{Fe}_{2.6} \mathrm{OS}_{1.75} \mathrm{Se}_{0.25}$ were measured with a wavelength $\lambda=0.457842 \AA$ and at 295 $\mathrm{K}$ and $90 \mathrm{~K}$.

Room temperature, lab-based x-ray powder diffraction measurements were performed at the University of Louisville, using a Bruker D8 Discover diffractometer with $\mathrm{Cu} \mathrm{K} \alpha$ radiation and a PANalytical Empyrean diffractometer with $\mathrm{Cu} \mathrm{K}$ - $\alpha$ radiation of $\lambda=1.54056 \AA$. The Rietveld refinements analysis for all x-ray powder diffraction data were performed using GSAS-II software. crystal lattice parameters, atomic positions, and other relevant parameter were refined during this process. 


\subsubsection{Neutron diffraction}

We have performed neutron powder diffraction experiments on $\mathrm{Ca}_{2} \mathrm{O}_{2} \mathrm{Fe}_{2.6} \mathrm{OS}_{2}$ and $\mathrm{Ca}_{2} \mathrm{O}_{2} \mathrm{Fe}_{2.6} \mathrm{OSS}_{1.75} \mathrm{Se}_{0.25}$ at the POWGEN beamline of SNS in ORNL. All samples were prepared inside a glovebox. We used a mortar and pestle to pulverize materials into a fine powder. Vanadium cans of $3 \mathrm{~mm}$ diameter were filled with approximately $3 \mathrm{~g}$ of powder sample. Figure 26 shows the sample preparation for the POWGEN experiment. For all measurements, we used a chopper frequency of $60 \mathrm{~Hz}$ with a wavelength center at $1.5 \AA$ with a minimum wavelength of $0.967 \AA$ and a maximum wavelength of $2.033 \AA$. This corresponds to $d$-spacings of $0.4854-14.5795 \AA$. Samples were measured at 10, 25, 50, 75, 100, 125, 150, 175, 200, 225, 250, 275 and 300 K approximately, for an hour each to get a full high-quality diffraction pattern. A temperature ramp measurement was performed on each sample as well. Each ramp measurement lasted about 5.8 hours. Seventy scans were collected where each scan lasted about 5 minutes. The ramp temperature started from $10 \mathrm{~K}$ up to $80 \mathrm{~K}$. All data were reduced using the Mantid Plot program [84].

\subsection{Results}

\subsubsection{Electrical Resistivity Measurements}

The temperature-dependent resistivity $\rho(T)$ measurements for $\mathrm{Ca}_{2} \mathrm{O}_{2} \mathrm{Fe}_{2.6} \mathrm{OS}_{2}$ and $\mathrm{Ca}_{2} \mathrm{O}_{2} \mathrm{Fe}_{2.6} \mathrm{OS}_{1.75} \mathrm{Se}_{0.25}$ are shown in Figure 27. The $\rho(T)$ for both materials has a negative coefficient of resistivity, which indicates a semiconducting behavior, as we expected from this material [11]. The magnitude of the resistivity at $300 \mathrm{~K}$ for $\mathrm{Ca}_{2} \mathrm{O}_{2} \mathrm{Fe}_{2.6} \mathrm{OS}_{2}$ is $6.02 \Omega \mathrm{cm}$ and for $\mathrm{Ca}_{2} \mathrm{O}_{2} \mathrm{Fe}_{2.6} \mathrm{OS}_{1.75} \mathrm{Se}_{0.25}$ is $0.53 \Omega \mathrm{cm}$.

The $\operatorname{Ln}(\rho)$ dependence with $\frac{1}{T}$ is shown in Figure 28 a) for the pure and b) for the doped sample. In the figure, both samples show a non-linear Arrhenius plots. In order

to calculate activation energy $E_{a}$ using the thermal activation model $\rho=\rho_{0} \exp \left(\frac{E_{a}}{K T}\right)$, 


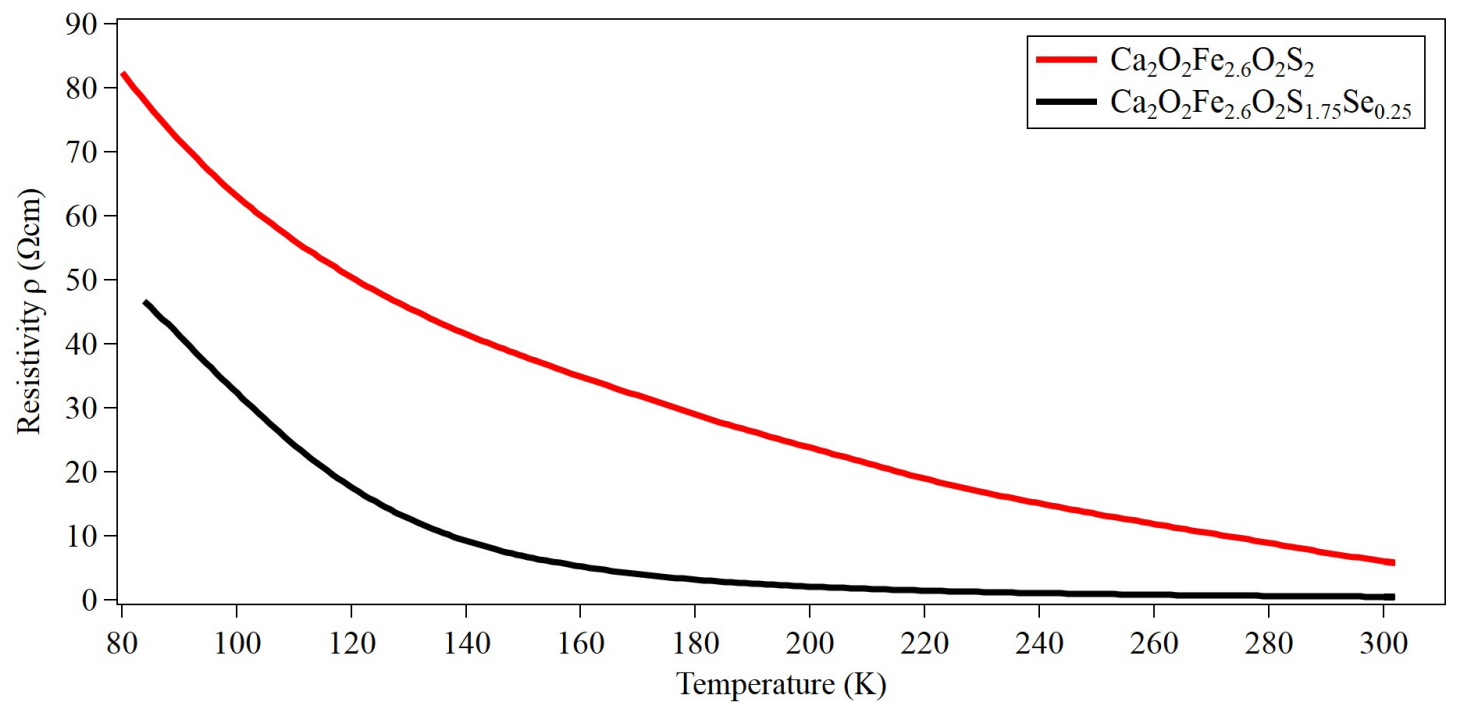

Figure 27. Temperature dependent of resistivity $\rho(T)$ for $\mathrm{Ca}_{2} \mathrm{O}_{2} \mathrm{Fe}_{2.6} \mathrm{OS}_{2-x} \mathrm{Se}_{x} \quad(x$ $=0,0.25)$.

a fit function $f(x)=a_{1}+a_{2} x$ was used to fit the temperature-dependent $\operatorname{Ln}(\rho)$ (Table 1). Sine those curves were non-linear, we selected 3 ranges where a linear fit would have better quality. For $\mathrm{Ca}_{2} \mathrm{O}_{2} \mathrm{Fe}_{2.6} \mathrm{OS}_{2}$ sample we used the following ranges: for fit one from 275.546 K to $299.73 \mathrm{~K}$, fit two from $205.198 \mathrm{~K}-262.289 \mathrm{~K}$ and fit three from $159.645 \mathrm{~K}$ to $196.65 \mathrm{~K}$.For $\mathrm{Ca}_{2} \mathrm{O}_{2} \mathrm{Fe}_{2.6} \mathrm{OS}_{1.75} \mathrm{Se}_{0.25}$ sample the fits were performed over the three temperature ranges: fit one from $249.576 \mathrm{~K}$ to $300.154 \mathrm{~K}$, fit two from 180.174 $\mathrm{K}$ to $244.887 \mathrm{~K}$ and fit three from $150.649 \mathrm{~K}$ to $175.261 \mathrm{~K}$. The values of activation energy $\mathrm{E}_{a}$, for both sample, increases with temperature (Table 1).

Table 1. Activation energy calculation from the Arrhenius plots in Figure 28 using the fit function $f(x)=a_{1}+a_{2} x$.

\begin{tabular}{lcccc}
\hline Temp. range (k) & fit param. $a_{o}$ & fit param. $a_{1}$ & $\chi^{2}$ & $\mathrm{E}_{a}(\mathrm{eV})$ \\
\hline \multicolumn{5}{c}{$\mathrm{Ca}_{2} \mathrm{O}_{2} \mathrm{Fe}_{2.6} \mathrm{OS}_{2}$} \\
$275.546-299.730$ & $-3.3451 \pm 0.0568$ & $1549.4 \pm 16.4$ & 0.0015 & $0.13352 \pm 0.00141$ \\
$205.198-262.289$ & $-0.12399 \pm 0.029$ & $679.12 \pm 7.02$ & 0.0024 & $0.05852 \pm 0.00060$ \\
$159.645-196.65$ & $1.6849 \pm 0.0239$ & $301.48 \pm 4.23$ & 0.0020 & $0.02598 \pm 0.00036$ \\
\hline \multicolumn{5}{c}{$\mathrm{Ca}_{2} \mathrm{O}_{2} \mathrm{Fe}_{2.6} \mathrm{OS}_{1.75} \mathrm{Se}_{0.25}$} \\
\hline $249.576-300.154$ & $-3.557 \pm 0.00535$ & $877.67 \pm 1.47$ & 0.0016 & $0.07563 \pm 0.00013$ \\
$180.174-244.887$ & $-3.1433 \pm 0.0074$ & $777.1 \pm 1.55$ & 0.0013 & $0.06697 \pm 0.00013$ \\
$150.649-175.261$ & $-2.6448 \pm 0.0273$ & $687.65 \pm 4.45$ & 0.0008 & $0.05926 \pm 0.00038$ \\
\hline
\end{tabular}



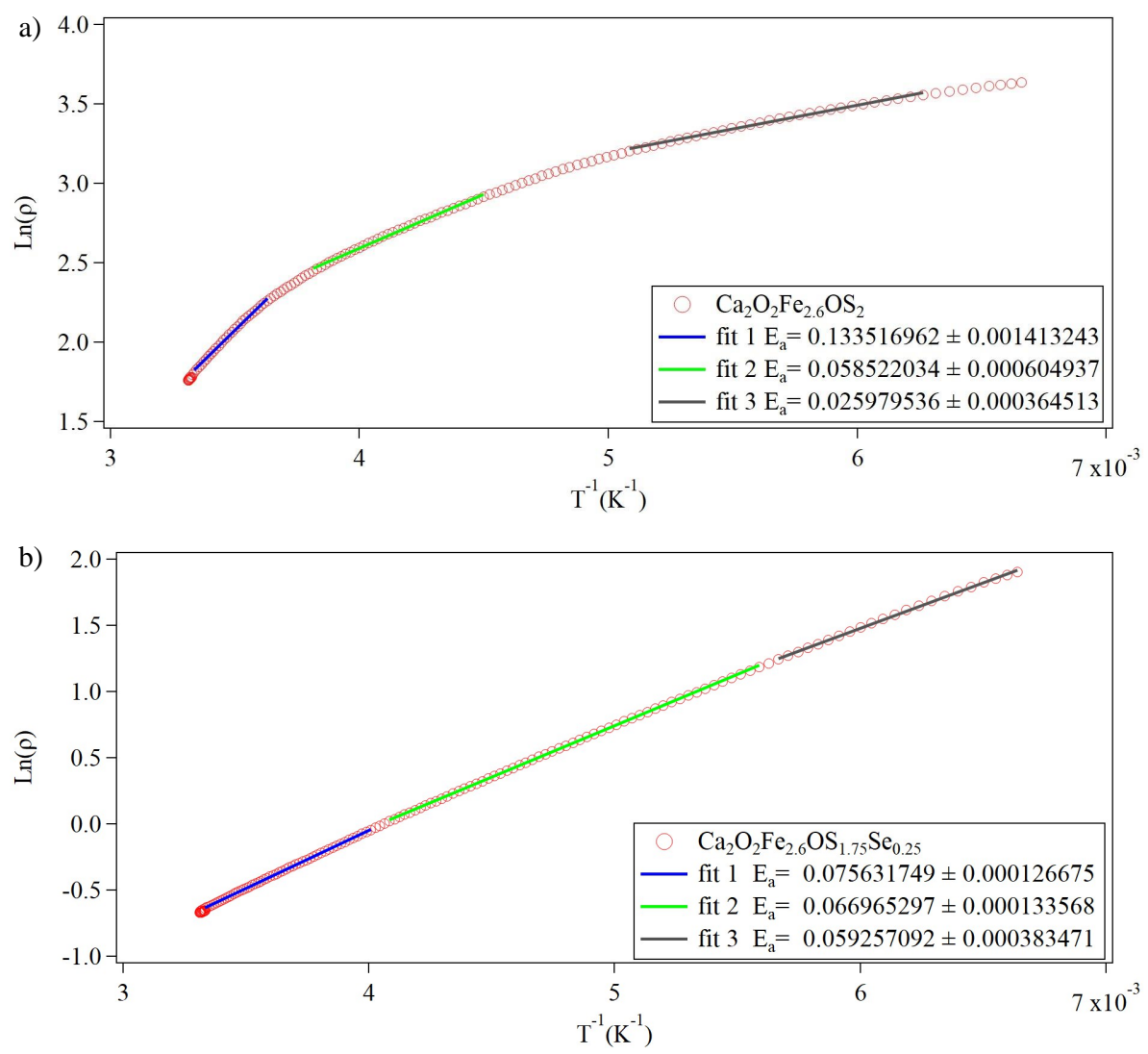

Figure 28. Resistivity measurement data above $150 \mathrm{~K}$ is used to fit the thermal activation model $\rho=\rho_{0} \exp \left(\frac{E_{a}}{K T}\right)$. Panel a) and b) show the fits for $\mathrm{Ca}_{2} \mathrm{O}_{2} \mathrm{Fe}_{2.6} \mathrm{OS}_{2}$ and $\mathrm{Ca}_{2} \mathrm{O}_{2} \mathrm{Fe}_{2.6} \mathrm{OS}_{1.75} \mathrm{Se}_{0.25}$, respectively.

The Mott activation energy was calculated, for the pure and doped material as shown in Figure 29 and Table 2, using the Mott variable range hopping model $\rho(T)=$ $\rho_{0} \exp \left(T_{0} / T\right)^{1 / 4}$. In Figure 29 the $\operatorname{Ln}(\rho)$ vs. $T^{-1 / 4}$ showed a non-linear curve when plotted for the temperature range from $\approx 80 \mathrm{~K}$ to $300 \mathrm{~K}$. The activation energy $E_{a}$ for $\mathrm{Ca}_{2} \mathrm{O}_{2} \mathrm{Fe}_{2.6} \mathrm{OS}_{2}$ sample was calculated for the most linear part of that curve, from 85.4 K - 140.9 K (Table 2 a) and its value was $1.38 \pm 0.004 \mathrm{meV}$. The doped sample also showed non-linear curve for $\operatorname{Ln}(\rho)$ vs. $T^{-1 / 4}$ which can be fitted in to two linear parts, the first with temperature from $84.91 \mathrm{~K}$ to $106.56 \mathrm{~K}$ and the second fit covered temperatures from $122.22 \mathrm{~K}$ to $241.28 \mathrm{~K}$. The calculated activation energy $E_{a}$ values for $\mathrm{Ca}_{2} \mathrm{O}_{2} \mathrm{Fe}_{2.6} \mathrm{OS}_{2}$ was $2.47 \pm 0.05 \mathrm{meV}$ for the first fit and $5.04 \pm 0.008 \mathrm{meV}$ for the second one. 

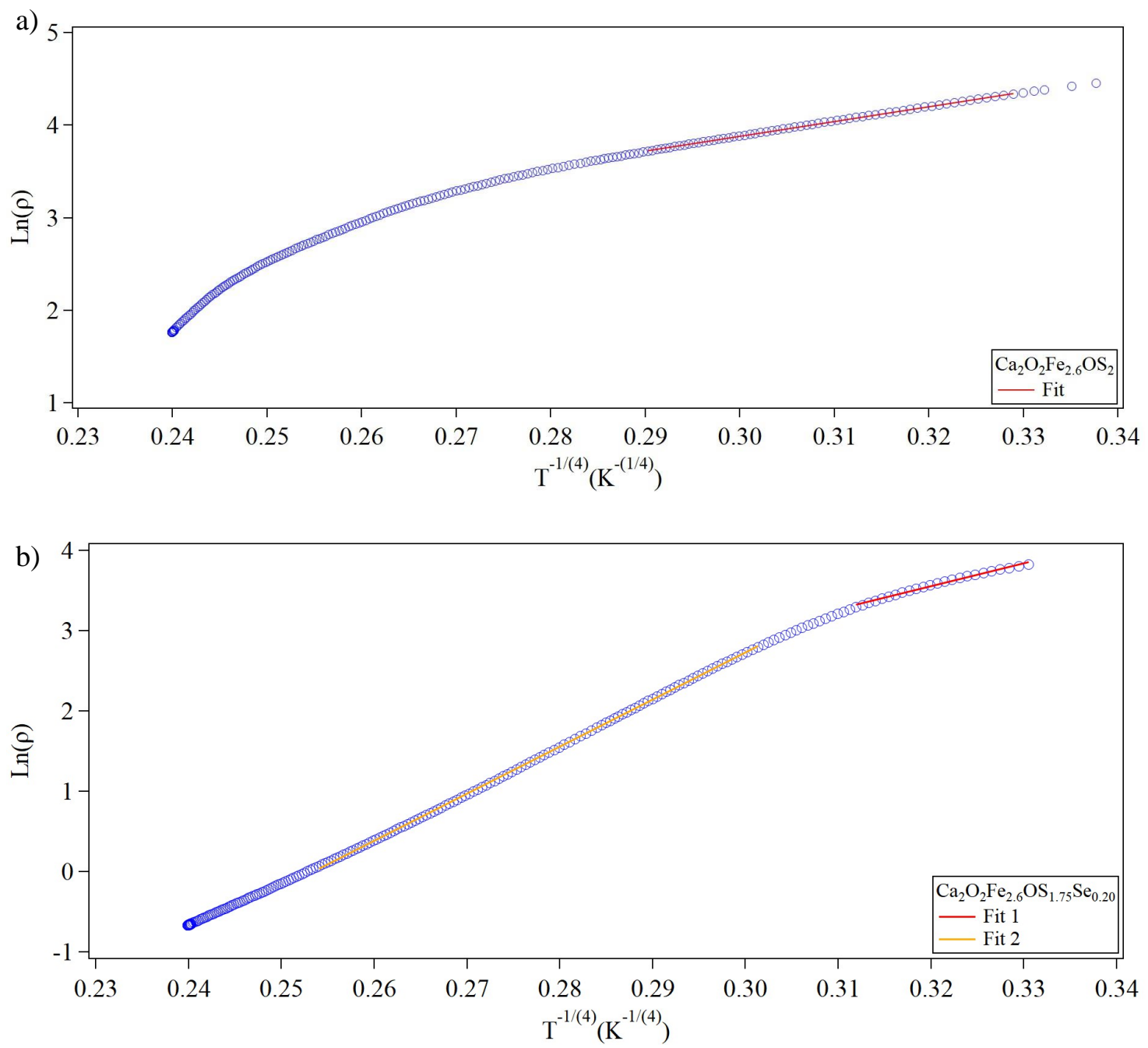

Figure 29. Temperature dependant resistivity measurements are fitted to variablerange hopping VRH model.

Table 2. VRH model activation energy calculation using a fit function $f(x)=$ $a_{1}+a_{2} x$ (Figure 29).

\begin{tabular}{lcccc}
\hline Temp. range (K) & fit param. $a_{o}$ & fit param $a_{1}$ & $\chi^{2}$ & $\mathrm{E}_{a}(\mathrm{eV})$ \\
\hline $\mathrm{Ca}_{2} \mathrm{O}_{2} \mathrm{Fe}_{2.6} \mathrm{OS}_{2}$ & & & & \\
\hline $85.433-140.9$ & $-0.91453 \pm 0.0152$ & $15.993 \pm 0.0495$ & 0.0011 & $0.00138 \pm 0.000004$ \\
\hline $\mathrm{Ca}_{2} \mathrm{O}_{2} \mathrm{Fe}_{2.6} \mathrm{OS}_{1.75} \mathrm{Se}_{0.25}$ & & & & \\
\hline $84.911-106.556$ & $-5.6284 \pm 0.19$ & $28.700 \pm 0.5940$ & 0.0057 & $0.00247 \pm 0.00005$ \\
$122.221-241.281$ & $-14.828 \pm 0.0226$ & $58.526 \pm 0.0820$ & 0.0146 & $0.00504 \pm 0.000008$ \\
\hline
\end{tabular}


a)

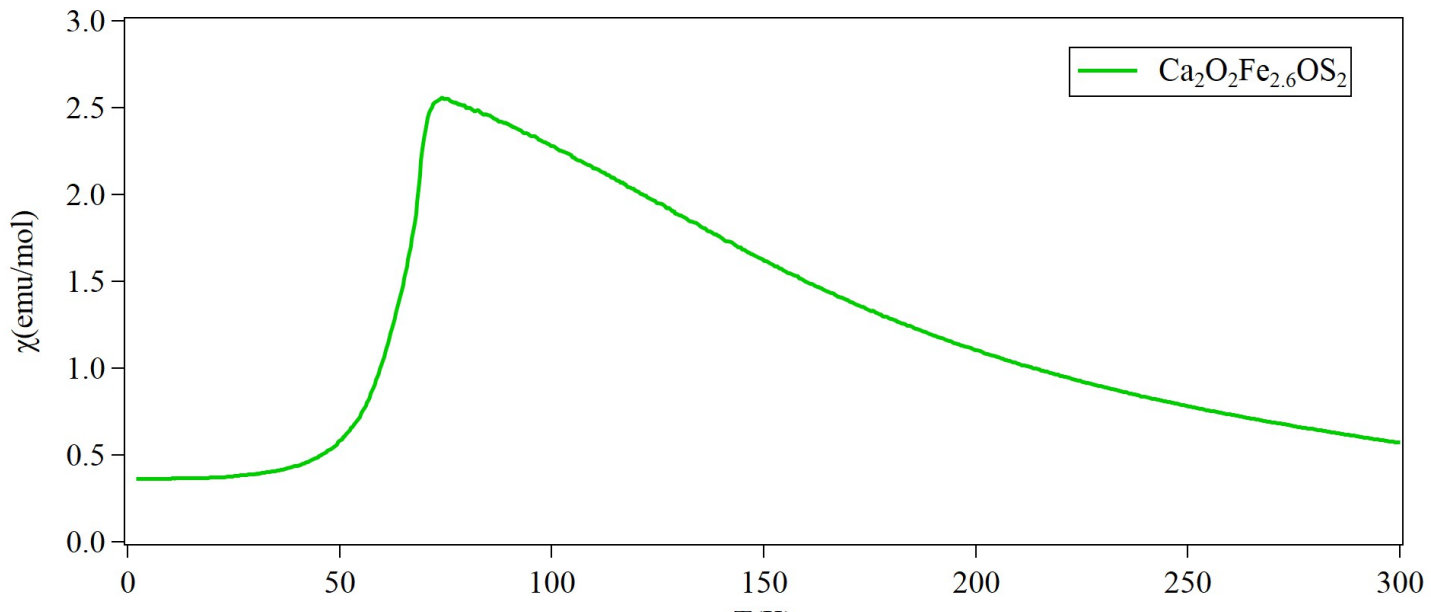

b)

$\mathrm{T}(\mathrm{K})$

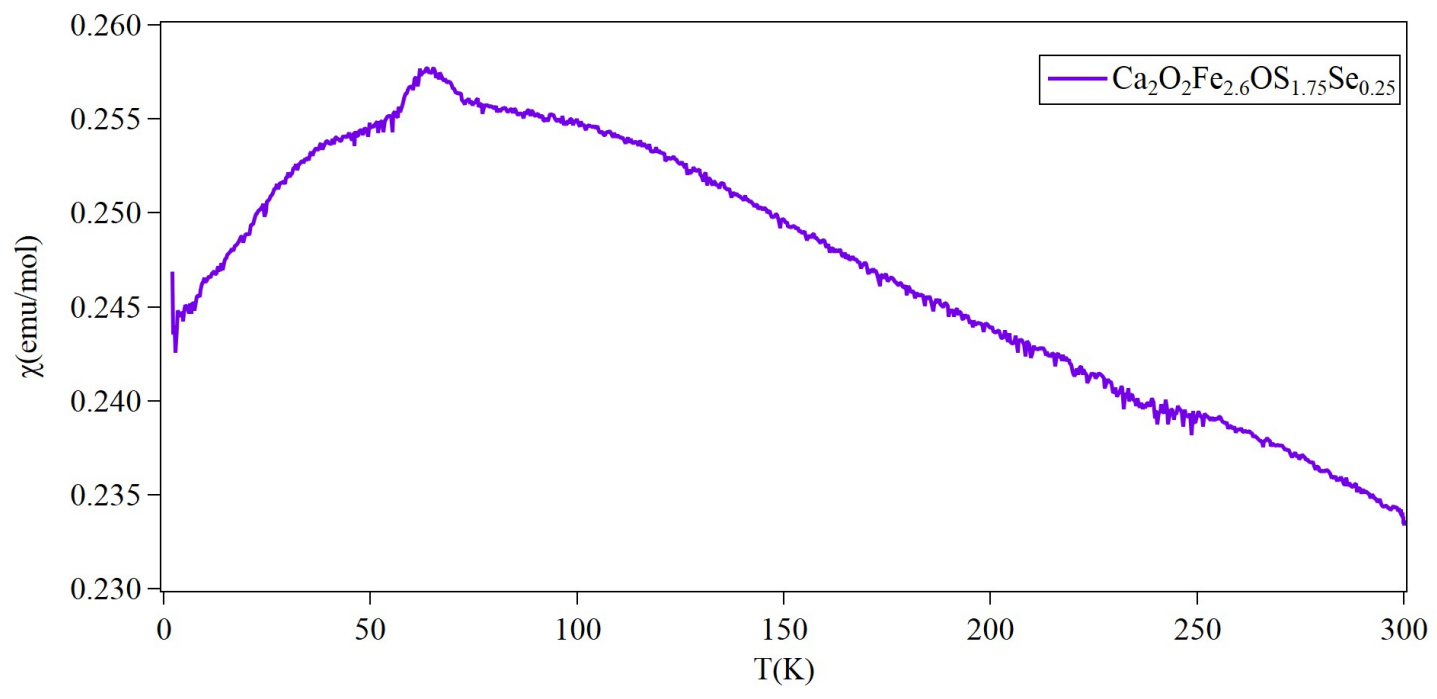

Figure 30. Temperature dependent magnetic susceptibility measurement showing antiferromagnetic behaviour for a) $\mathrm{Ca}_{2} \mathrm{O}_{2} \mathrm{Fe}_{2.6} \mathrm{OS}_{2}$, and b) $\mathrm{Ca}_{2} \mathrm{O}_{2} \mathrm{Fe}_{2.6} \mathrm{OS}_{1.75} \mathrm{Se}_{0.25}$.

\subsubsection{Magnetic Susceptibility Measurements}

The magnetic susceptibility measurements with respect to temperature was performed in an applied magnetic field of 1000 Oe. The susceptibility of the two materials behave differently with temperature. The $\mathrm{Ca}_{2} \mathrm{O}_{2} \mathrm{Fe}_{2.6} \mathrm{OS}_{2}$ susceptibility curve shows typical antiferromagnetic behaviour with a discontinuity at $74.092 \mathrm{~K}$, which indicates that a magnetic phase transition occurs at this temperature. The $\mathrm{Ca}_{2} \mathrm{O}_{2} \mathrm{Fe}_{2.6} \mathrm{OS}_{1.75} \mathrm{Se}_{0.25}$ susceptibility shows a broad maximum around $74 \mathrm{~K}$, with a 
small peak centered at $64 \mathrm{~K}$.

\subsubsection{X-ray Diffraction}

Figure 31 along with Tables 3 and 4 summarize the results of Rietveld refinement of lab based x-ray powder diffraction using GSAS-II software. The XRD was performed on both $\mathrm{Ca}_{2} \mathrm{O}_{2} \mathrm{Fe}_{2.6} \mathrm{OS}_{2}$ and $\mathrm{Ca}_{2} \mathrm{O}_{2} \mathrm{Fe}_{2.6} \mathrm{OS}_{1.75} \mathrm{Se}_{0.25}$ at room temperature with $\lambda=1.54 \AA$. The calculated pattern (shown in blue) fits well with the measured pattern for both samples with intensity mismatched in some peaks. (Figure 31). Also, the samples have a similar peak profile. However, the relative peak intensity differs between $\mathrm{Ca}_{2} \mathrm{O}_{2} \mathrm{Fe}_{2.6} \mathrm{OS}_{1.75} \mathrm{Se}_{0.25}$ and the pure $\mathrm{Ca}_{2} \mathrm{O}_{2} \mathrm{Fe}_{2.6} \mathrm{OS}_{2}$. In Tables 3 and 4, using the tetragonal symmetry and space group $P 4 / \mathrm{mmm}$ provides good fit for the XRD data as the $R_{w}$ and $\chi^{2}$ values are 0.041 and 0.015 for $\mathrm{Ca}_{2} \mathrm{O}_{2} \mathrm{Fe}_{2.6} \mathrm{OS}_{2}, 0.050$ and 0.014 for $\mathrm{Ca}_{2} \mathrm{O}_{2} \mathrm{Fe}_{2.6} \mathrm{OS}_{1.75} \mathrm{Se}_{0.25}$. The refined lattice parameters, the atomic positions,isotropic thermal displacement parameter $U_{\text {iso }}$ and atomic coordination are presented in those tables as well. During the Rietveld analysis, only the Fe atomic site occupancy was refine for the $\mathrm{Ca}_{2} \mathrm{O}_{2} \mathrm{Fe}_{2.6} \mathrm{OS}_{2}$ sample. For $\mathrm{Ca}_{2} \mathrm{O}_{2} \mathrm{Fe}_{2.6} \mathrm{OS}_{1.75} \mathrm{Se}_{0.25}$ sample Fe, S and Se atomic site occupancy were refine, and the sum of S and Se atomic site occupancy was set to one.

The x-ray powder diffraction patterns obtained at 11-BM, APS are displayed in Figures 32 and 33. Data were collected at $90 \mathrm{~K}$ and 295 K. In Figure 32, the 295 $\mathrm{K}$ measurement has higher intensity than the $90 \mathrm{~K}$ for the pure sample. On the other hand, the diffraction pattern at $295 \mathrm{~K}$ showed higher peak intensities the 90 $\mathrm{K}$ in the doped sample as shown in Figure 33. The diffraction experiment data were analyzed by Rietveld refinement method using GSAS-II software [85]. Tables 5 and 6 summarize the Rietveld analysis for $\mathrm{Ca}_{2} \mathrm{O}_{2} \mathrm{Fe}_{2.6} \mathrm{OS}_{2}$, while Tables 7 and 8 summarize the Rietveld analysis for $\mathrm{Ca}_{2} \mathrm{O}_{2} \mathrm{Fe}_{2.6} \mathrm{OS}_{1.75} \mathrm{Se}_{0.25}$. 

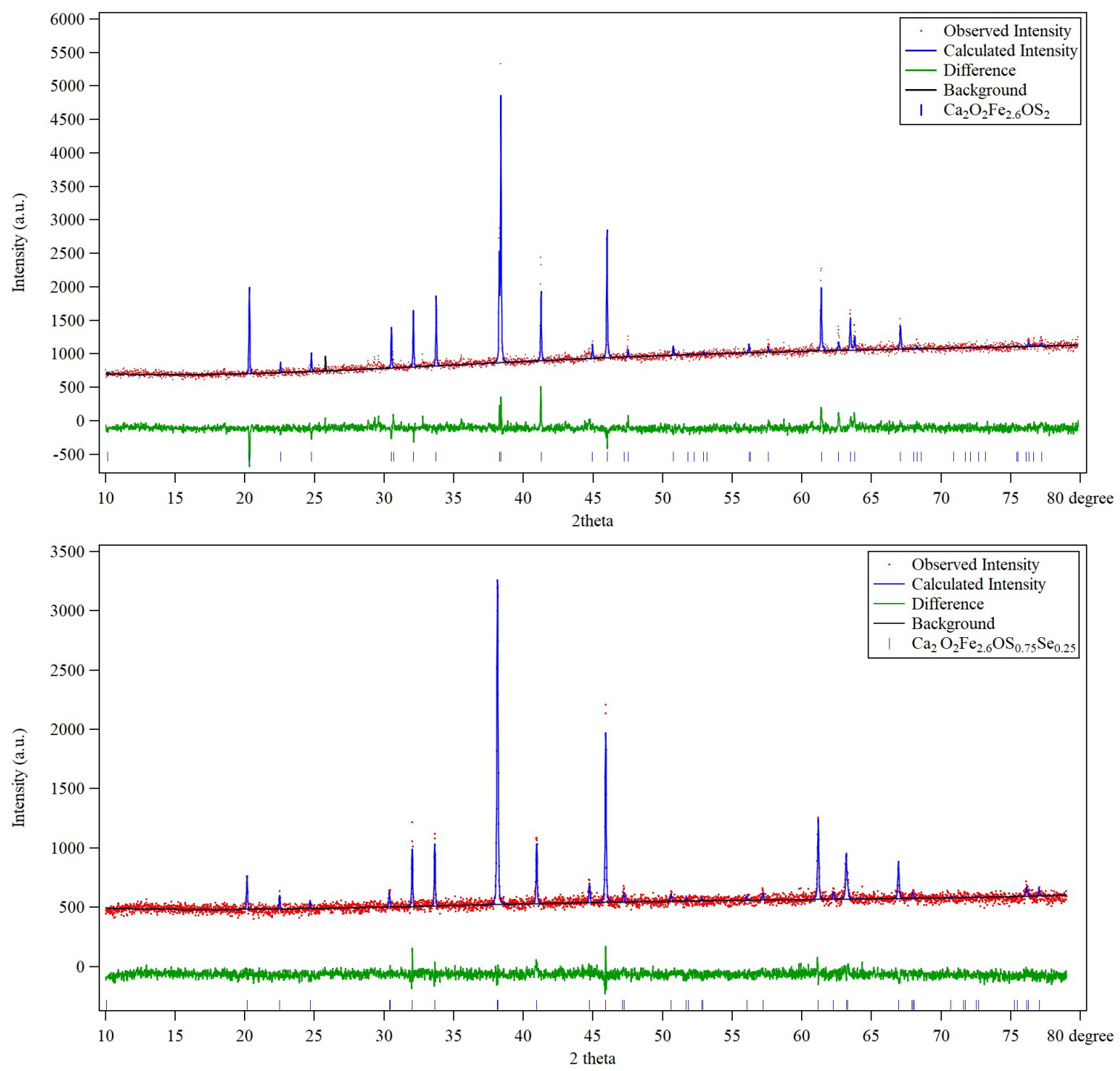

Figure 31. Rietveld Refinement profiles for the pure sample $\mathrm{Ca}_{2} \mathrm{O}_{2} \mathrm{Fe}_{2.6} \mathrm{OS}_{2}$ in the upper panel and the doped sampleCa $\mathrm{O}_{2} \mathrm{Fe}_{2.6} \mathrm{OS}_{1.75} \mathrm{Se}_{0.25}$ in the lower panel, from room temperature lab-based XRD. 
Table 3. Rietveld Refinement results for $\mathrm{Ca}_{2} \mathrm{O}_{2} \mathrm{Fe}_{2.6} \mathrm{OS}_{2}$ from room temperature lab-based XRD. Space Group P4/mmm $a=3.94485(5) \AA, c=8.7481(1) \AA$ Aunit cell volume $=136.138(3) \AA^{3}$.

\begin{tabular}{lccccc}
\hline \multicolumn{5}{c}{$\mathrm{Ca}_{2} \mathrm{O}_{2} \mathrm{Fe}_{2.6} \mathrm{OS}_{2}$} \\
\hline Atom & $\mathrm{x}$ & $\mathrm{y}$ & $\mathrm{z}$ & Occup. & $\mathrm{U}_{\text {iso }}\left(\AA^{2}\right)$ \\
$\mathrm{Ca}$ & 0.0 & 0.0 & $0.8063(8)$ & 1.0 & $0.009(3)$ \\
$\mathrm{O} 1$ & 0.5 & 0.0 & 1.0 & 1.0 & $0.041(9)$ \\
$\mathrm{O} 2$ & 0.0 & 0.0 & 0.5 & 1.0 & $0.002(1)$ \\
$\mathrm{Fe} 1$ & 0.5 & 0.5 & 1.0 & 1.0 & $0.001(3)$ \\
$\mathrm{Fe} 2$ & 0.5 & 0.0 & 0.5 & $0.73(1)$ & $0.027(5)$ \\
$\mathrm{S}$ & 0.5 & 0.5 & $0.7039(9)$ & 1.0 & $0.023(4)$
\end{tabular}

Coordination

$\begin{array}{lc}d_{F e 1-O 1}(\AA) & 1.97376(0) \\ d_{F e 2-O 2}(\AA) & 1.97376(0) \\ d_{F e 1-S}(\AA) & 2.6073(9) \\ d_{F e 2-S}(\AA) & 2.6564(6) \\ d_{F e 2-F e 2}(\AA) & 2.79132(0) \\ d_{C a-O 1}(\AA) & 2.5715(6) \\ a_{S-F e 2-S}(\text { deg. }) & 95.8(3) \\ a_{S-F e 2-S}(\text { deg. }) & 180.0(0) \\ a_{C a-O 1-C a}(\text { deg. }) & 98.7(3)\end{array}$

$\mathrm{wR}_{P}=0.041 \quad \mathrm{R}_{P}=0.053 \quad \mathrm{GoF}=1.24 \quad \chi^{2}=0.015$

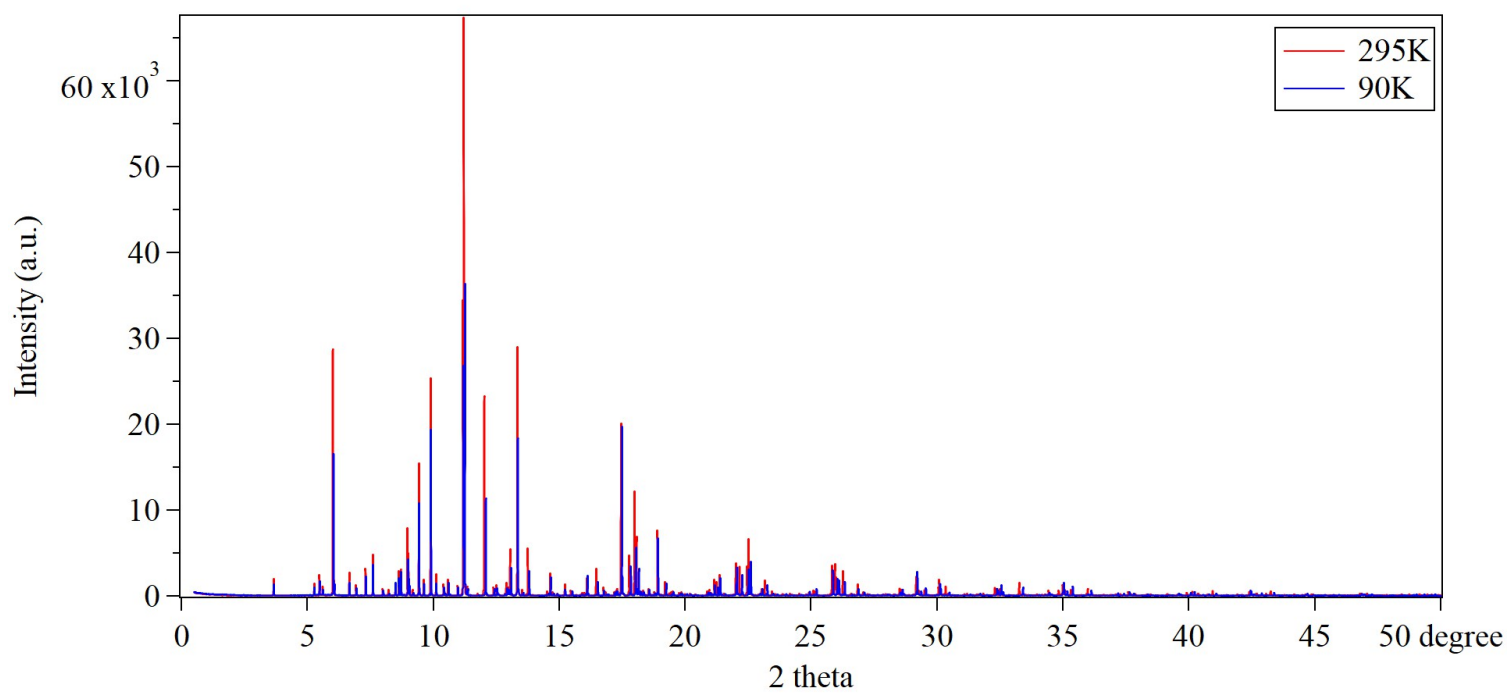

Figure 32. X-ray powder diffraction from 11-BM, APS of $\mathrm{Ca}_{2} \mathrm{O}_{2} \mathrm{Fe}_{2.6} \mathrm{OS}_{2}$ at temperatures $90 \mathrm{~K}$ and $295 \mathrm{~K}$. 
Table 4. Rietveld Refinement results for $\mathrm{Ca}_{2} \mathrm{O}_{2} \mathrm{Fe}_{2.6} \mathrm{OS}_{1.75} \mathrm{Se}_{0.25}$ from room temperature lab-based XRD. Space Group P4/mmm $a=3.95089(6) \AA, c=8.8094(2)$ Ånit cell volume $=137.510(4) \AA^{3}$.

\begin{tabular}{|c|c|c|c|c|c|}
\hline \multicolumn{4}{|c|}{$\mathrm{Ca}_{2} \mathrm{O}_{2} \mathrm{Fe}_{2.6} \mathrm{OS}_{1.75} \mathrm{Se}_{0.25}$} & & \\
\hline Atom & $\mathrm{x}$ & $\mathrm{y}$ & $\mathrm{Z}$ & Occup. & $\mathrm{U}_{\text {iso }}\left(\AA^{2}\right)$ \\
\hline $\mathrm{Ca}$ & 0.0 & 0.0 & $0.805(1)$ & 1.0 & $0.0482(58)$ \\
\hline $\mathrm{O} 1$ & 0.5 & 0.0 & 1.0 & 1.0 & $0.0044(96)$ \\
\hline $\mathrm{O} 2$ & 0.0 & 0.0 & 0.5 & 1.0 & $0.0091(151)$ \\
\hline Fe1 & 0.5 & 0.5 & 1.0 & 1.0 & $0.0218(56)$ \\
\hline $\mathrm{Fe} 2$ & 0.5 & 0.0 & 0.5 & $0.76(1)$ & $0.0442(76)$ \\
\hline S & 0.5 & 0.5 & $0.702(1)$ & $0.86(3)$ & $0.0757(93)$ \\
\hline Se & 0.5 & 0.5 & $0.702(1)$ & $0.12(3)$ & $0.0121(33)$ \\
\hline
\end{tabular}

Coordination

$\begin{array}{lc}d_{F e 1-O 1}(\AA) & 1.9754(0) \\ d_{F e 2-O 2}(\AA) & 1.9754(0) \\ d_{F e 1-S}(\AA) & 2.623(10) \\ d_{F e 2-S}(\AA) & 2.661(7) \\ d_{F e 2-F e}(\AA) & 2.7937(0) \\ d_{C a-O 1}(\AA) & 2.617(7) \\ a_{S-F e 2-S}(\text { deg. }) & 95.9(4) \\ a_{O 2-F e 2-O 2}(\text { deg. }) & 180.0(0) \\ a_{C a-O 1-C a}(\text { deg. }) & 98.0(4)\end{array}$

$\mathrm{wR}_{P}=0.0495 \quad \mathrm{R}_{P}=0.057 \quad \mathrm{GoF}=1.17 \quad \chi^{2}=0.014$

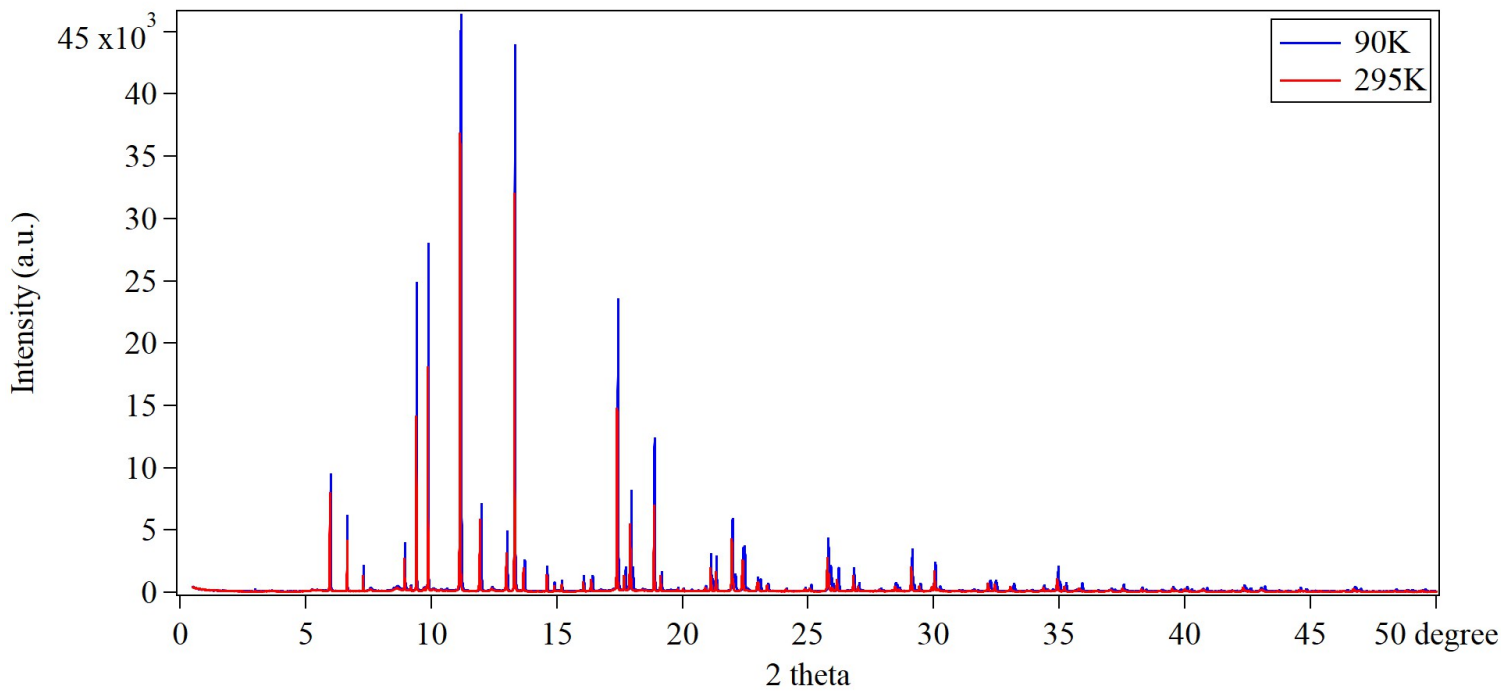

Figure 33. X-ray powder diffraction from 11-BM, APS of $\mathrm{Ca}_{2} \mathrm{O}_{2} \mathrm{Fe}_{2.6} \mathrm{OS}_{1.75} \mathrm{Se}_{0.25}$ at temperatures $90 \mathrm{~K}$ and $295 \mathrm{~K}$. 
Table 5. Proposed model for $\mathrm{Ca}_{2} \mathrm{O}_{2} \mathrm{Fe}_{2.6} \mathrm{OS}_{2}$ from 11-BM XRD at 295K. Space Group P4/mmm $a=3.94552(1) \AA, c=8.75195(2)$ Åunit cell volume $=136.243(1)$ $\AA^{3}$.

\begin{tabular}{|c|c|c|c|c|c|}
\hline \multicolumn{4}{|c|}{$\mathrm{Ca}_{2} \mathrm{O}_{2} \mathrm{Fe}_{2.6} \mathrm{OS}_{2}$} & & \\
\hline Atom & $\mathrm{x}$ & $\mathrm{y}$ & Z & Occup. & $\mathrm{U}_{\text {iso }}\left(\AA^{2}\right)$ \\
\hline $\mathrm{Ca}$ & 0.0 & 0.0 & $0.8127(1)$ & $0.986(2)$ & $0.0072(3)$ \\
\hline $\mathrm{O} 1$ & 0.5 & 0.0 & 1.0 & $0.832(6)$ & $0.0025(9)$ \\
\hline $\mathrm{O} 2$ & 0.0 & 0.0 & 0.5 & $0.946(10)$ & $0.0085(15)$ \\
\hline Fe1 & 0.5 & 0.5 & 1.0 & $0.995(2)$ & $0.0089(3)$ \\
\hline $\mathrm{Fe} 2$ & 0.5 & 0.0 & 0.5 & $0.746(2)$ & $0.0193(3)$ \\
\hline S & 0.5 & 0.5 & $0.7049(1)$ & $0.951(3)$ & $0.0056(3)$ \\
\hline
\end{tabular}

Coordination

$\begin{array}{lc}d_{F e 1-O 1}(\AA) & 1.97276(0) \\ d_{F e 2-O 2}(\AA) & 1.97276(0) \\ d_{F e 1-S}(\AA) & 2.5827(11) \\ d_{F e 2-S}(\AA) & 2.6660(8) \\ d_{F e 2-F e e}(\AA) & 2.78990(0) \\ d_{C a-O 1}(\AA) & 2.5652(6) \\ a_{S-F e 2-S}(\text { deg. }) & 95.46(4) \\ a_{O 2-F e 2-O 2}(\text { deg. }) & 180.0(0) \\ a_{C a-O 1-C a}(\text { deg. }) & 100.54(3)\end{array}$

$\mathrm{wR}_{P}=0.2685 \quad \mathrm{R}_{P}=0.1850 \quad \mathrm{GoF}=4.64 \quad \chi^{2}=0.2152$ 
Table 6. Proposed model for $\mathrm{Ca}_{2} \mathrm{O}_{2} \mathrm{Fe}_{2.6} \mathrm{OS}_{2}$ from 11-BM XRD at 90K. Space Group $P 4 / m m m a=3.94091(1) \AA, c=8.70806(3)$ Åunit cell volume $=135.243(1) \AA^{3}$.

\begin{tabular}{|c|c|c|c|c|c|}
\hline \multicolumn{4}{|c|}{$\mathrm{Ca}_{2} \mathrm{O}_{2} \mathrm{Fe}_{2.6} \mathrm{OS}_{2}$} & & \\
\hline Atom & $\mathrm{x}$ & $\mathrm{y}$ & $\mathrm{Z}$ & Occup. & $\mathrm{U}_{\text {iso }}\left(\AA^{2}\right)$ \\
\hline $\mathrm{Ca}$ & 0.0 & 0.0 & $0.8116(1)$ & $0.800(2)$ & $0.0003(2)$ \\
\hline O1 & 0.5 & 0.0 & 1.0 & $0.805(8)$ & $0.0152(13)$ \\
\hline $\mathrm{O} 2$ & 0.0 & 0.0 & 0.5 & $0.760(10)$ & $0.0165(20)$ \\
\hline Fe1 & 0.5 & 0.5 & 1.0 & $0.814(2)$ & $0.0008(3)$ \\
\hline $\mathrm{Fe} 2$ & 0.5 & 0.0 & 0.5 & $0.627(2)$ & $0.0175(4)$ \\
\hline S & 0.5 & 0.5 & $0.7041(1)$ & $0.778(3)$ & $0.0006(3)$ \\
\hline
\end{tabular}

Coordination

$\begin{array}{lc}d_{F e 1-O 1}(\AA) & 1.97045(0) \\ d_{F e 2-O 2}(\AA) & 1.97045(0) \\ d_{F e 1-S}(\AA) & 2.5765(12) \\ d_{F e 2-S}(\AA) & 2.6538(8) \\ d_{F e 2-F e e}(\AA) & 2.78664(0) \\ d_{C a-O 1}(\AA) & 2.5642(6) \\ a_{S-F e 2-S}(\text { deg. }) & 95.89(4) \\ a_{O 2-F e 2-O 2}(\text { deg. }) & 180.0(0) \\ a_{C a-O 1-C a}(\text { deg. }) & 100.43(3)\end{array}$

$\mathrm{wR}_{P}=0.2685 \quad \mathrm{R}_{P}=0.1850 \quad \mathrm{GoF}=4.64 \quad \chi^{2}=0.2152$ 
Table 7. Proposed model for $\mathrm{Ca}_{2} \mathrm{O}_{2} \mathrm{Fe}_{2.6} \mathrm{OS}_{1.75} \mathrm{Se}_{0.25}$ from 11-BM XRD at 295K. Space Group P4/mmm $a=3.952598(1) \AA, c=8.813372(2) \AA$, unit cell volume = $137.692(1) \AA^{3}$.

\begin{tabular}{|c|c|c|c|c|c|}
\hline \multicolumn{4}{|c|}{$\mathrm{Ca}_{2} \mathrm{O}_{2} \mathrm{Fe}_{2.6} \mathrm{OS}_{1.75} \mathrm{Se}_{0.25}$} & & \\
\hline Atom & $\mathrm{x}$ & $\mathrm{y}$ & $\mathrm{Z}$ & Occup. & $\mathrm{U}_{i s o}\left(\AA^{2}\right)$ \\
\hline $\mathrm{Ca}$ & 0.0 & 0.0 & $0.8139(1)$ & $0.990(37)$ & $0.0079(2)$ \\
\hline O1 & 0.5 & 0.0 & 1.0 & $0.967(35)$ & $0.0171(10)$ \\
\hline $\mathrm{O} 2$ & 0.0 & 0.0 & 0.5 & $0.905(33)$ & $0.0058(13)$ \\
\hline Fe1 & 0.5 & 0.5 & 1.0 & $0.988(37)$ & $0.0044(3)$ \\
\hline $\mathrm{Fe} 2$ & 0.5 & 0.0 & 0.5 & $0.774(29)$ & $0.0247(3)$ \\
\hline S & 0.5 & 0.5 & $0.7034(1)$ & $0.890(32)$ & $0.0162(65)$ \\
\hline Se & 0.5 & 0.5 & $0.7034(1)$ & $0.110(32)$ & $0.0023(18)$ \\
\hline
\end{tabular}

Coordination

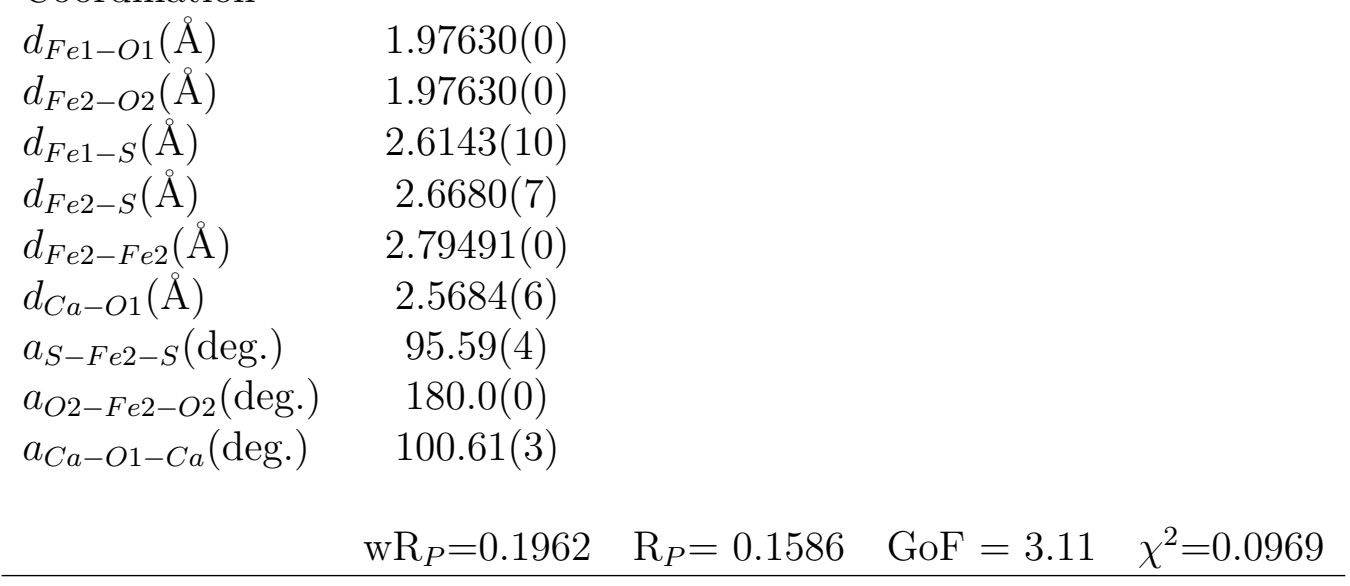


Table 8. Proposed model for $\mathrm{Ca}_{2} \mathrm{O}_{2} \mathrm{Fe}_{2.6} \mathrm{OS}_{1.75} \mathrm{Se}_{0.25}$ from 11-BM XRD at 90K. Space Group P4/mmm $a=3.94752(1) \AA, c=8.77023(2) \AA$, unit cell volume $=136.666(1)$ $\AA^{3}$.

\begin{tabular}{lccccc}
\hline \multicolumn{7}{c}{$\mathrm{Ca}_{2} \mathrm{O}_{2} \mathrm{Fe}_{2.6} \mathrm{OS}_{1.75} \mathrm{Se}_{0.25}$} & & & \\
\hline Atom & $\mathrm{x}$ & $\mathrm{y}$ & $\mathrm{z}$ & Occup. & $\mathrm{U}_{\text {iso }}\left(\AA^{2}\right)$ \\
$\mathrm{Ca}$ & 0.0 & 0.0 & $0.8121(1)$ & $0.949(32)$ & $0.0082(2)$ \\
$\mathrm{O} 1$ & 0.5 & 0.0 & 1.0 & $0.971(32)$ & $0.0218(10)$ \\
$\mathrm{O} 2$ & 0.0 & 0.0 & 0.5 & $0.857(29)$ & $0.0002(11)$ \\
$\mathrm{Fe} 1$ & 0.5 & 0.5 & 1.0 & $0.923(31)$ & $0.0064(3)$ \\
$\mathrm{Fe} 2$ & 0.5 & 0.0 & 0.5 & $0.732(25)$ & $0.0239(3)$ \\
$\mathrm{S}$ & 0.5 & 0.5 & $0.7027(1)$ & $0.925(28)$ & $0.0155(88)$ \\
$\mathrm{Se}$ & 0.5 & 0.5 & $0.7027(1)$ & $0.075(28)$ & $0.0033(16)$
\end{tabular}

Coordination

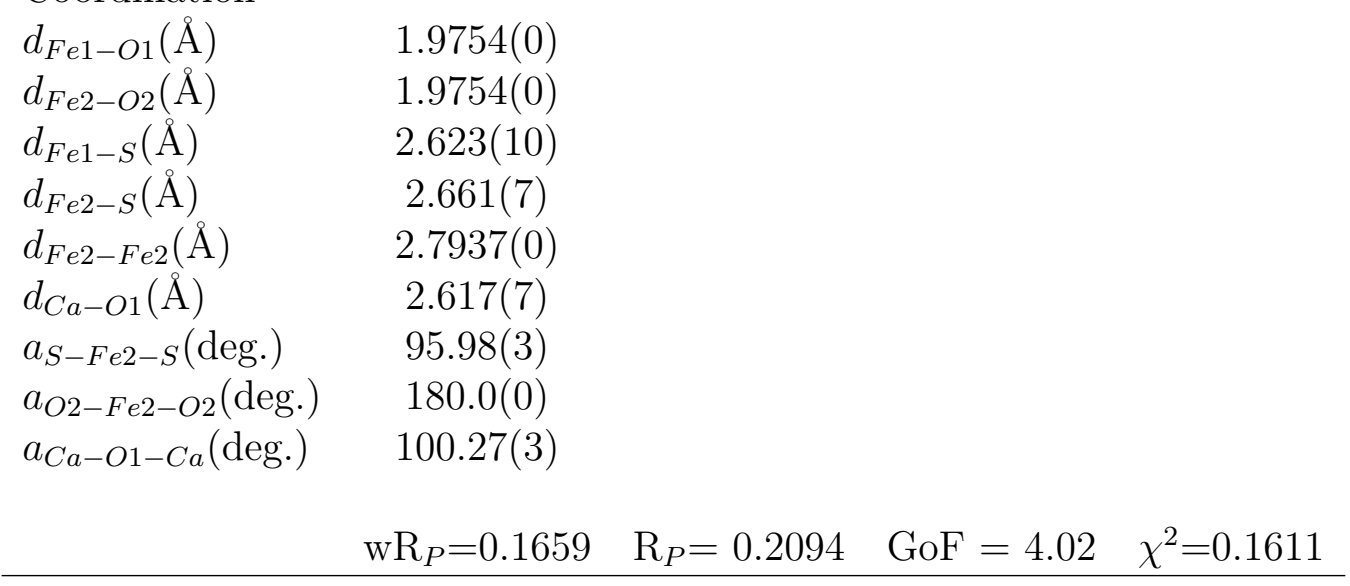




\subsubsection{Neutron Powder Diffraction and Order Parameter}

Temperature dependent neutron powder diffraction patterns of $\mathrm{Ca}_{2} \mathrm{O}_{2} \mathrm{Fe}_{2.6} \mathrm{OS}_{2}$ are shown in Figure 34. Three magnetic peaks can be identified by comparing high temperature to lower temperature diffraction patterns. The highest intensity is the peak located at T.O.F. $=1.3179 \times 10^{+5} \mu s$ (intensity $=157.6$ ), the second highest intensity peak is at T.O.F. $=86709 \mu s$ (intensity $=80$ ) and the weakest magnetic peak intensity is at T.O.F. $=79535 \mu s$ (intensity $=84$ ).

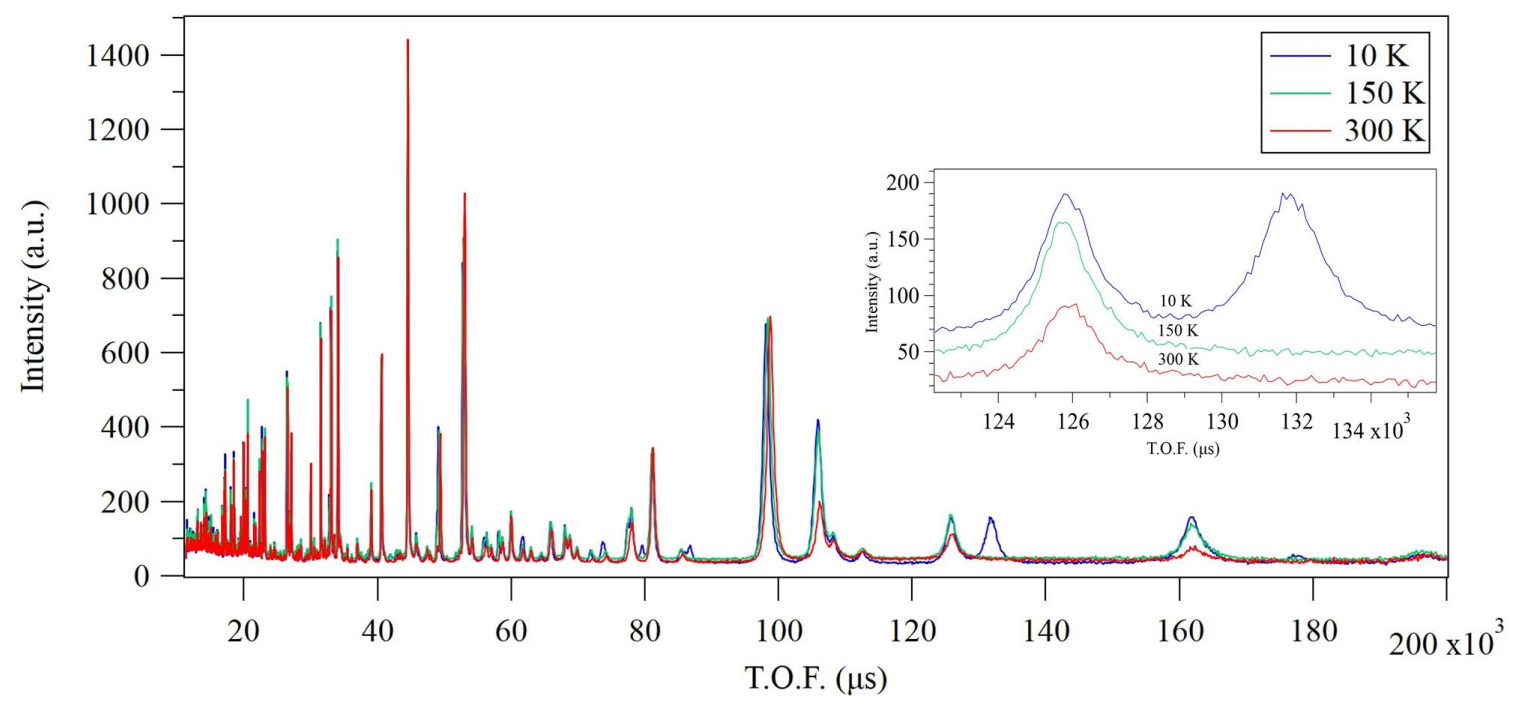

Figure 34. Neutron powder diffraction from POWGEN of $\mathrm{Ca}_{2} \mathrm{O}_{2} \mathrm{Fe}_{2.6} \mathrm{OS}_{2}$ at temperatures $10 \mathrm{~K}, 150 \mathrm{~K}$ and $300 \mathrm{~K}$.the insert show the evolution of the most intense magnetic peak with temperature.

Figure 35 shows the temperature dependence neutron powder diffraction for the sample $\mathrm{Ca}_{2} \mathrm{O}_{2} \mathrm{Fe}_{2.6} \mathrm{OS}_{1.75} \mathrm{Se}_{0.25}$. There are five magnetic peaks that can be identified. The highest intensity peak is located at T.O.F. $=1.324 \times 10^{+5} \mu s$ (highest intensity $=$ 114.3), followed by the peak at T.O.F. $=61988 \mu s$ (intensity $=104.7$ ), then at T.O.F $=79619 \mu s$ (intensity $=100.8)$, then the peak at T.O.F $=73912 \mu s$ (intensity $=$ 82.5), and the lowest magnetic peak is located at T.O.F. $86523 \mu s$ with intensity $=$ 75.6 . 


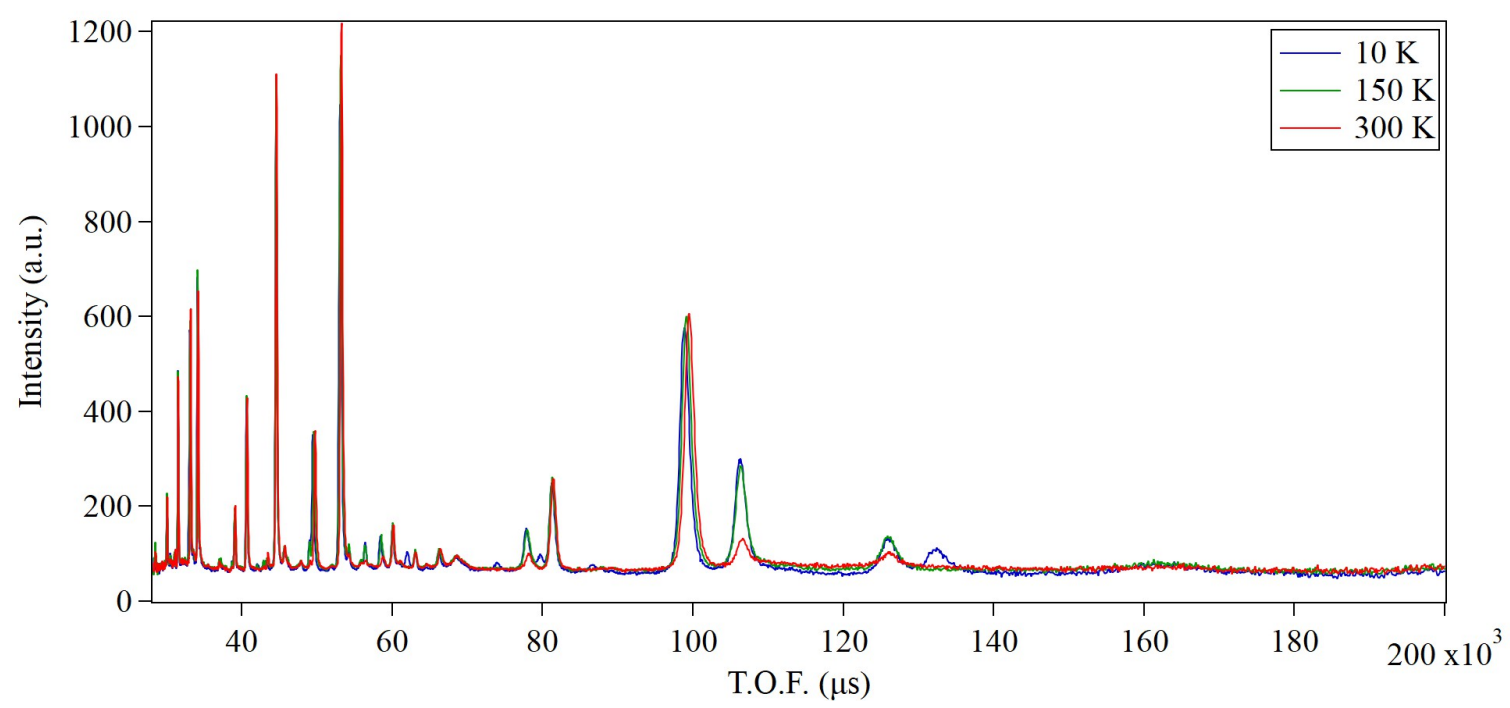

Figure 35. Neutron powder diffraction from POWGEN of $\mathrm{Ca}_{2} \mathrm{O}_{2} \mathrm{Fe}_{2.6} \mathrm{OS}_{1.75} \mathrm{Se}_{0.25}$ at temperatures $10 \mathrm{~K}, 150 \mathrm{~K}$ and $300 \mathrm{~K}$.

We have performed neutron powder diffraction during temperature ramps of $2 \mathrm{~K}$ steps at POWGEN beamline, ORNL. This experiment was aimed to calculated the magnetic order parameter for $\mathrm{Ca}_{2} \mathrm{O}_{2} \mathrm{Fe}_{2.6} \mathrm{OS}_{2-x} \mathrm{Se}_{x}$ samples. For $\mathrm{Ca}_{2} \mathrm{O}_{2} \mathrm{Fe}_{2.6} \mathrm{OS}_{2}$ sample, Figure 36 shows the result of order parameter fit analysis. The red dots represent the height of the selected magnetic peak with respect to changes in temperature. We have used equation $f(T)=c_{1}\left(1-\frac{T}{c_{2}}\right)^{c 3}$ for performing the fit analysis. The fit parameters for $\mathrm{Ca}_{2} \mathrm{O}_{2} \mathrm{Fe}_{2.6} \mathrm{OS}_{2}$ were: $c_{1}=179.64 \pm 2.35, c_{2}=75.01 \pm 0.0194$, and $c_{3}$ $=0.24553 \pm 0.0127$. 


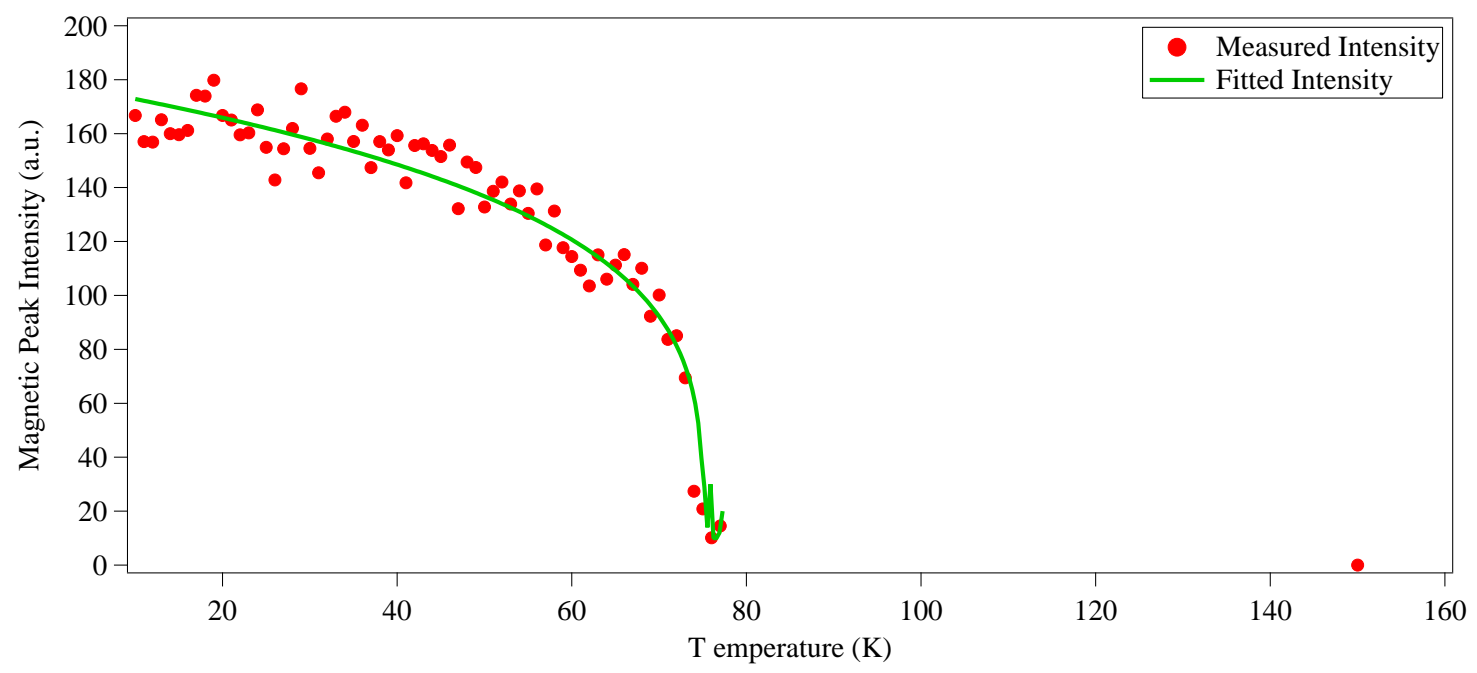

Figure 36. Order parameter fitting for $\mathrm{Ca}_{2} \mathrm{O}_{2} \mathrm{Fe}_{2.6} \mathrm{OS}_{2}$ sample based on temperature dependence neutron powder diffraction calculated from the magnetic peak at T.O.F. $1.324 \times 10^{+5} \mu s\left(d_{h k l}=4.364 \AA\right)$. The order parameter describes the development of magnetic order. In $\mathrm{Ca}_{2} \mathrm{O}_{2} \mathrm{Fe}_{2.6} \mathrm{OS}_{2}$ the continuous magnetic phase transition is from the paramagnetic phase for $T>T_{N}$ to the antiferromagnetic phase when $T<T_{N}$. The magnetic transition temperature $T_{N}$ based on this fitting is $75.01 \pm 0.0194 \mathrm{~K}$.

\subsection{Discussion}

\subsubsection{Electrical Resistivity Measurements}

Transition metal compounds usually display saturated resistivity, which has a substantial decrease in the slope of the resistivity as a function of temperature [73]. The temperature-dependent resistivity for both materials shows a semiconducting behavior (Figure 27), although the pure sample is one order of magnitude higher than the doped sample, signifying that the electron are more localized in the doped sample compared to the pure one [28]. The resistivity at $300 \mathrm{~K}$ for $\mathrm{Ca}_{2} \mathrm{O}_{2} \mathrm{Fe}_{2.6} \mathrm{OS}_{2}$ is 6.02 $\Omega \mathrm{cm}$ and for $\mathrm{Ca}_{2} \mathrm{O}_{2} \mathrm{Fe}_{2.6} \mathrm{OS}_{1.75} \mathrm{Se}_{0.25}$ is $0.53 \Omega \mathrm{cm}$ are well beyond the Mott-Ioffe-Regel (MIR). The measurements of the pure and doped samples have many similarities to the reported by Zhang et al. [11]. Our resistivity measurement curvature differs from what Zhang et al. results reported [11], and the values for their resistivity at temperatures higher than $250 \mathrm{~K}$ are more elevated than our measurements. 
The calculated activation energy $\mathrm{E}_{a}$ for the pure sample shows a temperature dependence trend; it decreased with temperature. The doped sample shows the same trend with more consistent values of activation energy. Based activation energy $\mathrm{E}_{a}$ and the semiconducting behavior, the band gap $\left(\mathrm{E}_{g}\right)$ can be assumed to be $\left(\mathrm{E}_{g}\right)=2 \mathrm{E}_{a}$ yielding small band gap $\left(\mathrm{E}_{g}\right)$ below $14 \mathrm{meV}$ for both material over all temperature ranges (Table 1) [74]. In the studied material doping with Se increase the lattice parameter and the unit cell volume while having a complex behavior with the activation energy. The activation energy at higher temperature (the first temp. range in Table 1 and Figure 28) decreases in value with doping. The average calculated activation energy $E_{a}$ for $\mathrm{Ca}_{2} \mathrm{O}_{2} \mathrm{Fe}_{2.6} \mathrm{OS}_{2}$ was $0.07267 \pm 0.00079 \mathrm{eV}$ which is comparable to the reported value of $0.073 \mathrm{eV}[11]$. For the doped sample $\mathrm{Ca}_{2} \mathrm{O}_{2} \mathrm{Fe}_{2.6} \mathrm{OS}_{1.75} \mathrm{Se}_{0.25}$ the activation energy $E_{a}$ was $0.06728 \pm 0.00021 \mathrm{eV}$. For $\mathrm{La}_{2} \mathrm{O}_{2} \mathrm{Fe}_{2} \mathrm{O} M_{2}$, which is similar structurally to $\mathrm{Ca}_{2} \mathrm{O}_{2} \mathrm{Fe}_{2.6} \mathrm{OS}_{2}$ and $\mathrm{Ca}_{2} \mathrm{O}_{2} \mathrm{Fe}_{2.6} \mathrm{OS}_{1.75} \mathrm{Se}_{0.25}$, the activation energy were $0.19 \mathrm{eV}$ and $0.24 \mathrm{eV}$, for $M=\mathrm{Se}, \mathrm{S}$ respectively [13].

Activation energy $E_{a}$ values for some iron oxychalcogenides are presented in Table 9. The $\mathrm{Ca}_{2} \mathrm{O}_{2} \mathrm{Fe}_{2.6} \mathrm{OS}_{2}$ and $\mathrm{Ca}_{2} \mathrm{O}_{2} \mathrm{Fe}_{2.6} \mathrm{OS}_{1.75} \mathrm{Se}_{0.25}$ materials which have the lowest $E_{a}$ values compared to the other compounds.

Table 9. Activation energy values and critical exponent for the magnetic order parameter for some iron oxychalcogenides. The values for our $\mathrm{E}_{a}$ values are the average values for all temperature ranges from Table 1.

\begin{tabular}{lccc}
\hline Compound & Activation Energy $(\mathrm{eV})$ & $\beta$ & Reference \\
\hline $\mathrm{La}_{2} \mathrm{O}_{2} \mathrm{Fe}_{2} \mathrm{OSe}_{2}$ & 0.19 & 0.129 & {$[14,9]$} \\
$\mathrm{La}_{2} \mathrm{O}_{2} \mathrm{Fe}_{2} \mathrm{OS}_{2}$ & 0.24 & 0.133 & {$[14,9]$} \\
$\mathrm{Ce}_{2} \mathrm{O}_{2} \mathrm{FeOSe}_{2}$ & 0.32 & 0.28 & {$[14]$} \\
$\mathrm{Ce}_{2} \mathrm{O}_{2} \mathrm{Fe}_{2} \mathrm{OSe}_{2}$ & 0.26 & 0.11 & {$[14]$} \\
$\mathrm{Ca}_{2} \mathrm{O}_{2} \mathrm{Fe}_{2.6} \mathrm{OS}_{2}$ & 0.073 & - & {$[11]$} \\
$\mathrm{Ca}_{2} \mathrm{O}_{2} \mathrm{Fe}_{2.6} \mathrm{OS}_{2}$ & $0.07267 \pm 0.00079$ & 0.123 & This work \\
$\mathrm{Ca}_{2} \mathrm{O}_{2} \mathrm{Fe}_{2.6} \mathrm{OS}_{1.75} \mathrm{Se}_{0.25}$ & $0.06728 \pm 0.00021$ & - & This work \\
\hline
\end{tabular}




\subsubsection{Magnetic Susceptibility Measurements}

The pure sample magnetic susceptibility (Figure 30 a) shows an antiferromagnetic behavior with a discontinuity at $74 \mathrm{~K}$ indicating the magnetic phase transition temperature for the pure sample which is lower than the reported $77 \mathrm{~K}$ [11]. The presence of a sharp peak in the magnetic susceptibility indicates long-range AFM phase transition and it is different than the other iron oxychalcogenides that usually show a broad maxima $[11,9,77,10]$. The broadening of the magnetic susceptibility maximum and its deviation from Curie-Weiss law indicates the strong antiferromagnetic short-range correlations. This behavior is similar to $\mathrm{La}_{2} \mathrm{O}_{2} \mathrm{Fe}_{2} \mathrm{OSe}_{2}$ [9] and $\mathrm{Na}_{2} \mathrm{Fe}_{2} \mathrm{Se}_{2} \mathrm{O}$ and indicates a 2D magnetic system [77]. Figure 20 shows the temperature dependence magnetic susceptibility in iron oxychalcogenides where all materials have the characteristic broad peak. $\mathrm{La}_{2} \mathrm{O}_{2} \mathrm{Fe}_{2} \mathrm{OSe}_{2}$ and $\mathrm{La}_{2} \mathrm{O}_{2} \mathrm{Fe}_{2} \mathrm{OS}_{2}$, panels a and b in Figure 20 respectively, and panel c shows both $\mathrm{Sr}_{2} \mathrm{~F}_{2} \mathrm{Fe}_{2} \mathrm{OS}_{2}$ and $\mathrm{Ba}_{2} \mathrm{~F}_{2} \mathrm{Fe}_{2} \mathrm{OS}_{2}$ with the former having a broader peak than the latter. Zhang et al. [11] studied the affect of doping on the $\mathrm{Ca}_{2} \mathrm{O}_{2} \mathrm{Fe}_{2.6} \mathrm{OS}_{2}$ material and found that doping suppress the long-range magnetic ordering due to the increase of randomness in Fe2 vacancies with doping.

\subsubsection{X-ray Diffraction}

In this study we performed a detailed nuclear structural study of the Mott insulator $\mathrm{Ca}_{2} \mathrm{O}_{2} \mathrm{Fe}_{2.6} \mathrm{OS}_{2-x} \mathrm{Se}_{x}(x=0,0.25)$. We have performed, two x-ray powder diffraction experiments. The first used a lab-based x-ray diffractometer and the second used synchrotron x-ray at at 11-BM, APS. The lab-based XRD enabled us to characterize our samples. $\mathrm{Ca}_{2} \mathrm{O}_{2} \mathrm{Fe}_{2.6} \mathrm{OS}_{2-x} \mathrm{Se}_{x}$ samples were measured at room temperature by a $1.54 \AA$ wavelength lab based x-ray. The results of the Rietveld analysis are shown in Figure 31 and Tables 3 and 4 . We have chosen the tetragonal crystal system with space group $P 4 / m m m$ to perform the Rietveld analysis. From the anal- 
ysis we have concluded that the chosen crystal structure, and symmetry, fits well the measured diffraction pattern. The quality of the Rietveld analysis is illustrated in Figure 31 and the values of fit parameters $\left(R_{w}, \chi^{2}\right)$ in Table 3 and 4 . Zhang et al.[11] reported powder x-ray diffraction for $\mathrm{Ca}_{2} \mathrm{O}_{2} \mathrm{Fe}_{2.6} \mathrm{OS}_{2}$ and concluded that tetragonal system with space group $P 4 / \mathrm{mmm}$ best fit their data. They have found some unfitted peaks in their analysis and concluded that they might need to consider a super-cell with $\sqrt{8} a \times 2 c$. For our lab-based XRD analysis we did not consider any superlattice because the Rietveld analysis fits had good quality without the need to include a superlattice in the refinement.

In order to further investigate the structure of this material with better precision, we conducted a high resolution x-ray powder diffraction experiment at 11BM, APS. Both materials were measured with $\lambda=0.4578 \AA$ at room temperature and at $90 \mathrm{~K}$. The peaks in the XRD data at $90 \mathrm{~K}$ for $\mathrm{Ca}_{2} \mathrm{O}_{2} \mathrm{Fe}_{2.6} \mathrm{OS}_{2}$ have lower intensity than the room temperature as in Figure 32. Studying the behaviour of $\mathrm{Ca}_{2} \mathrm{O}_{2} \mathrm{Fe}_{2.6} \mathrm{OS}_{1.75} \mathrm{Se}_{0.25} \mathrm{XRD}$, we found that the peaks sharpen at low temperature compared to room temperature as expected.

Attempts to perform Rietveld fitting for $\mathrm{Ca}_{2} \mathrm{O}_{2} \mathrm{Fe}_{2.6} \mathrm{OS}_{2-x} \mathrm{Se}_{x}$ samples was not trivial. The fit quality represented by $R_{w}$ and $\chi^{2}$ values in Tables 5-8, were worse than our fits from the lab-based analysis. A probable explanation for this finding is that the high resolution synchrotron diffraction data had many smaller peaks that were not visible in the lab x-ray due to the presence of superlattice. Also, the synchrotron x-ray have greater penetration which can lead to greater scattering intensity.

The presented model to describe the crystal structure of $\mathrm{Ca}_{2} \mathrm{O}_{2} \mathrm{Fe}_{2.6} \mathrm{OS}_{2-x} \mathrm{Se}_{x}$ resulted in poor Rietveld fits (Tables 5-8). This can be due to the presence of a superlattice, as suggested by Zhang et al. [11] , contamination during the synthesis process, or the need to model the disorder in this material better and include this to represent a better-fitted crystal model to the measured diffraction data. 
In order to investigate the reported superlattice structure presence in the pure $\mathrm{Ca}_{2} \mathrm{O}_{2} \mathrm{Fe}_{2.6} \mathrm{OS}_{2}$ material, we performed the indexing procedure and Pawley [86] refinement using GSAS-II software. We could not determine the reported [11] superlattice using the mentioned procedures by using Lab-based x-ray data. When using the high-resolution synchrotron data, we confirmed the presence of a supercell with space group $P 4 / m m m$ and lattice parameters $a=11.13888 \AA, c=17.47045 \AA$, and unit cell volume $=2167.638390 \AA^{3}$. The superlattice only present in the pure sample. Our investigation in the doped sample did not provide us with any structure. The presence of iron $\mathrm{Fe} 2$ vacancies has been linked to the superlattice structure in the pure samples. Zhang et al. [11] studied how the ordered Fe2 vacancies promote the presence of the superlattice. The evolution of superlattice peaks (at about $23.4^{\circ}$ and $24.8^{\circ}$ Figure 6 from reference [11]) with doping indicated that the superlattice peaks intensity decrease with increasing of the Se doping, which lead to their conclusion that the Se doping diminishes the superlattice related peaks in the XRD pattern.

\subsubsection{Neutron Powder Diffraction and Magnetic Order Parameter}

A temperature dependent neutron powder diffraction experiment was conducted at POWGEN beamline, ORNL. Diffraction patterns were obtained in $2 \mathrm{~K}$ steps from the base temperature up to $80 \mathrm{~K}$ to study the behavior of the magnetic peaks. The magnetic order parameter is proportional to the peak with the highest detected intensity, located at T.O.F. $=1.324 \times 10^{+5} \mu s$, was used to perform the order parameter calculation. It is possible to study the magnetism within the sample because the neutron has a magnetic moment and interacts with the electrons moments around the atom. We can detect magnetic Bragg peaks caused by the arrangement of the magnetic atoms. The results of temperature ramps measurements were used to calculate the magnetic order parameter. Our magnetic order parameter results (Figure 36) showed that of the order parameter has a $\beta=0.12277 \pm 0.0064$. Our $\beta$ is 
very close to $1 / 8$ value that corresponds to the two dimensional antiferromagnetic Ising model $[87,9]$. This critical exponent value is similar to the values reported for $\mathrm{La}_{2} \mathrm{O}_{2} \mathrm{Fe}_{2} \mathrm{O}(\mathrm{S}, \mathrm{Se})_{2}$ and other Fe-based materials $[9,14]$ (see Table 9. The magnetic phase transition temperature based on the magnetic peak fit analysis is about $75 \mathrm{~K}$, which is similar to the value obtained from the magnetic susceptibility measurement. For the doped sample, the analysis of the magnetic order parameter did not revel any ordering.

\subsection{Conclusion}

Electrical resistivity measurement, magnetic susceptibility measurement, x-ray diffraction, and neutron diffraction were the main techniques used in this study. The high resolution powdered x-ray, and neutron diffraction experiments are among the first on $\mathrm{Ca}_{2} \mathrm{O}_{2} \mathrm{Fe}_{2.6} \mathrm{OS}_{2-x} \mathrm{Se}_{x}$. High resolution x-ray diffraction is a powerful tool to identify the crystal structure and symmetry. The current study Rietveld analysis showed that the tetragonal structure with space group $P 4 / m m m$ was the best crystal structure to fit our materials. The unit cell volume increased with temperature, while the atomic site occupancies increased with decrease in temperatures. Also, the Fe2 atom had the lowest site occupancy which is equivalent to vacancies in the $\left[\mathrm{Fe}_{2} \mathrm{OS}_{2}\right]^{2-}$ plane about $20 \%$ to $30 \%$, the presence of Fe2 vacancies was expected and reported for the pure sample [11], our measurements confirmed the presence of those vacancies for the pure and the doped sample.

On the other hand, temperature dependent neutron diffraction was used to study the magnetic order parameter of $\mathrm{Ca}_{2} \mathrm{O}_{2} \mathrm{Fe}_{2.6} \mathrm{OS}_{2}$. From the fit of the magnetic peak intensity with respect to temperature, magnetic phase transition temperature was determined to be at $75 \mathrm{~K}$. Using the same analysis, $\beta=0.12277 \pm 0.0064$ was determined and it corresponded to the two dimensional antiferromagnetic Ising model.

The $\mathrm{Ca}_{2} \mathrm{O}_{2} \mathrm{Fe}_{2.6} \mathrm{OS}_{2-x} \mathrm{Se}_{x}$ materials showed interesting features at low tempera- 
tures. The unit cell volume of the sample increased as temperature decreased, and the atomic site occupancy increased. These two observation are most likely related since the increase in the unit cell volume will encompass larger numbers of atoms. The presence of AFM ordering in the Fe atoms might affected the crystal structure at low temperature. Although the doping did not affect the conducting behavior it had a greater impact on the magnetic susceptibility suppressed the magnetic ordering greatly.

\subsection{Limitations and Future Work}

One of the possible next steps is to study the magnetic structure in details for the $\mathrm{Ca}_{2} \mathrm{O}_{2} \mathrm{Fe}_{2.6} \mathrm{OS}_{2-x} \mathrm{Se}_{x}$ Mott insulators . There is currently limited understanding of the magnetic phase and magnetic structure of $\mathrm{Ca}_{2} \mathrm{O}_{2} \mathrm{Fe}_{2.6} \mathrm{OS}_{2-x} \mathrm{Se}_{x}$ which challenge our understanding of the Mott insulators behavior. The current work should support a detailed magnetic Rietveld refinement. The affect of doping on the magnetic structure to confirm the transport measurement across dopings. A limitation of the present study is the presence of vacancies in the atomic sites. These vacancies affected the quality of the Rietveld analysis fits. In future work, the vacancies can be modeled and implemented into the fit analysis process. 


\section{CHAPTER 6}

\section{LOCAL STRUCTURE OF LANTHANUM IRON OXYCHALCOGINIDES}

\subsection{Introduction}

Superconductivity is one of the most exciting physics phenomena. Since the discovery of superconductivity, about a century ago, many researchers have worked to understand this phenomenon and the material that exhibits this property. Iron-based superconductors (FeSCs) had attracted tremendous attention among the superconducting materials because they have high-transition $T_{c}$ temperatures. In FeScs, the superconductivity is generated through the pairing mechanism of electron-electron Coulomb interaction. By comparison, conventional superconductors have a different superconductivity mechanism. In the conventional superconductors, the electronphonon coupling initiates the superconductivity phase [88, 89]. FeSCs and cuprates share many similarities in their phase diagram, both High- $T_{c}$ superconductors show the superconductivity in the vicinity of other competing phases like the nematic phase and spin-density-wave in FeSCs. Despite these similarities, FeSCs different compared to the cuprates in many aspects like the metallic phase of the parent, the conduction layer crystal structure, and the spin symmetry of the antiferromagnetic order[90].

In order to understand the nature of the superconductivity mechanism, it is essential to know how the superconductivity arises in FeSCs systems. Previously it was thought that magnetic fluctuations were the underlying origin of superconductivity since it arises near the magnetically ordered state. However, a more recent study revealed that there is another non-superconducting state, also called a nematic, besides 
the magnetism [91]. Since the superconductivity in the cuprates superconductors are based on the electron or hole doping into a strongly correlated Mott insulating phase [92], it is crucial to study the Mott-insulating phase of iron superconductors in order to understand the mechanism of high-temperature superconductivity in these systems.

Nematicity is the condition of being nematic, or extent, to which a material is nematic. It was first observed in ordered crystalline liquids and in solid materials nematicity was seen in superconducting materials phase diagram before the superconducting phase. Mazin et al. [93] stated that "Nematic phases are frequently found in organic matter. The defining characteristic of these phases is orientational order in the absence of long-range positional order, resulting in distinctive uniaxial physical properties. It has also been proposed that nematic order exists in some electronic systems, and may even play a role in mediating high-temperature superconductivity". The breaking of the electronic symmetry compared to the lattice is a distinguishing feature of electronic nematic phases. Therefore, nematic behavior can be defined as the existence of anisotropy in a property that locally breaks the symmetry of the crystal and/or reciprocal lattice structure but globally preserves it [94]. Strong evidence strongly leads to the electronic mechanism of nematicity, placing nematic order in the class of correlation-driven electronic instabilities [17].

Superconductivity originates near an antiferromagnetic (AFM) order, in both iron pnictides and iron chalcogenides. It is affected by the magnetism and the bad metal state [28]. The bad metal behavior is due to the proximity to a Mott transition. Song et al. [28] studied the Mott insulator $\mathrm{NaFe}_{0.5} \mathrm{Cu}_{0.5} \mathrm{As}$ and suggested that the electron correlations are sufficient to place it in proximity to Mott transition. They concluded that in iron pnictides and iron chalcogenides, the high-temperature superconducting transitions are induced by the electron correlations, bad-metal behavior, and the magnetism. In this work, the local structure of the Mott insulating iron 
oxychalcogenides $\mathrm{La}_{2} \mathrm{O}_{2} \mathrm{Fe}_{2} \mathrm{O}(\mathrm{S} \text {, Se })_{2}$ was studied. $\mathrm{La}_{2} \mathrm{O}_{2} \mathrm{Fe}_{2} \mathrm{OSe}_{2}$ has been described as a Mott insulator, and theoretical work suggested that it is more strongly correlated than $\mathrm{LaFeAsO}$ [95]. $\mathrm{La}_{2} \mathrm{O}_{2} \mathrm{Fe}_{2} \mathrm{OS}_{2}$ show Mott insulating properties due to the narrowing of the Fe $d$-electron band and correlation effects [77].

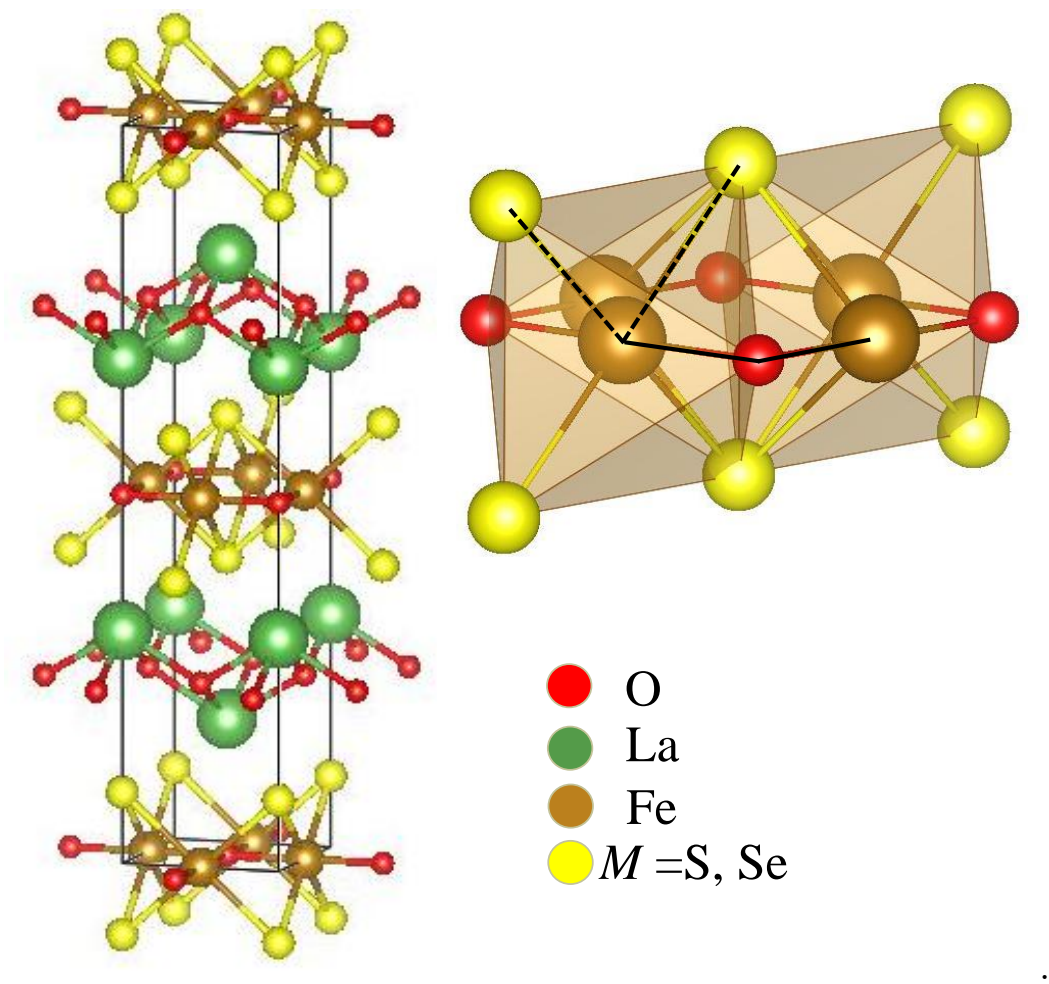

Figure 37. Crystal structure of lanthanum iron oxychalcogenide (left) and the octahedra (right), where the Fe atom is surrounded by four Se atoms and two O2 atoms.

Mayer et al. was the first to report the antiferromagnetic (AFM) insulator $\mathrm{La}_{2} \mathrm{O}_{2} \mathrm{Fe}_{2} \mathrm{O}(\mathrm{S}, \mathrm{Se})_{2}$ [96]. These iron oxychalcogenides are layered mixed anion materials containing more than one anionic species in a single phase. $\mathrm{La}_{2} \mathrm{O}_{2} \mathrm{Fe}_{2} \mathrm{O}(\mathrm{S}$, $\mathrm{Se})_{2}$ layers consists of a body-centered tetragonal crystal structure $(I 4 / \mathrm{mmm})$ with Fluorite-like $\left[\mathrm{La}_{2} \mathrm{O}_{2}\right]^{2+}$ layers and $\left[\mathrm{Fe}_{2} \mathrm{O}\right]^{2+}$ sheets separated by $M^{2-}$ anions where $M$ $=\mathrm{S}$ or Se. In this structure, $\left[\mathrm{Fe}_{2} \mathrm{O}\right]^{2+}$ consists of anti- $\mathrm{CuO}_{2}$ arrangement with $\mathrm{Fe}^{2+}$ cations is four coordinated by $M^{2-}$ (above and below the plane) and two in-plane oxygens, forming a tilted Fe-centered $\mathrm{FeO}_{2} \mathrm{Se}_{4}$ tilted octahedra [96, 95, 97]. Similar 
to $\mathrm{LaFeAsO}$ and FeSe, the Fe plane has approximately $90^{\circ} \mathrm{Fe}-\mathrm{Se}-\mathrm{Fe}$ intersections. However, the $\mathrm{Fe}$ plane contains additional $\mathrm{O}^{2-}$ Ions [95]. Figure 37 shows the crystal structure of $\mathrm{La}_{2} \mathrm{O}_{2} \mathrm{Fe}_{2} \mathrm{OSe}_{2}$ and its octahedra. The $\mathrm{La}_{2} \mathrm{O}_{2} \mathrm{Fe}_{2} \mathrm{O}(\mathrm{S}, \mathrm{Se})_{2}$ does not endure a structural phase transition at low temperatures.

$\mathrm{La}_{2} \mathrm{O}_{2} \mathrm{Fe}_{2} \mathrm{O}(\mathrm{S}, \mathrm{Se})_{2}$ are structurally similar to parent compounds of iron pnictides, and it was thought that $\mathrm{La}_{2} \mathrm{O}_{2} \mathrm{Fe}_{2} \mathrm{OS}_{2}$ material could be driven into superconductivity through doping. To date, this was not achieved experimentally. The magnetic structure for $\mathrm{La}_{2} \mathrm{O}_{2} \mathrm{Fe}_{2} \mathrm{O}(\mathrm{S}, \mathrm{Se})_{2}$ was studied intensively $[98,99,79,9]$. The magnetic order temperature, also called Néel temperature $\left(T_{N}\right)$, is the temperature above which an antiferromagnetic material becomes paramagnetic. It is the temperature at which the thermal energy becomes large enough to destroy the microscopic magnetic ordering within a material. In the $\mathrm{La}_{2} \mathrm{O}_{2} \mathrm{Fe}_{2} \mathrm{O}(\mathrm{S}, \mathrm{Se})_{2}$, the transition is from antiferromagnetic (AFM) to paramagnetic (PM) ordering is at $T_{N}=90.2 \mathrm{~K}$ for $M=$ Se and $T_{N}=107.1 \mathrm{~K}$ for $M=\mathrm{S}[9]$. The AFM magnetic structure of $\mathrm{La}_{2} \mathrm{O}_{2} \mathrm{Fe}_{2} \mathrm{O}(\mathrm{S}$, $\mathrm{Se})_{2}$ adopts a multicomponent 2- $k$ magnetic structure [95].

The purpose of this investigation was to explore the local structure of the Mott insulators $\mathrm{La}_{2} \mathrm{O}_{2} \mathrm{Fe}_{2} \mathrm{O}(\mathrm{S}, \mathrm{Se})_{2}$. Neutron powder diffraction (NPD) and the pair distribution function (PDF) technique were used to study the relationship between the structure and magnetism in those materials.

\subsection{Experimental Methods}

\subsubsection{Synthesis}

$\mathrm{La}_{2} \mathrm{O}_{2} \mathrm{Fe}_{2} \mathrm{O}(\mathrm{S}, \mathrm{Se})_{2}$ were synthesized at the Beijin National Laboratory for Condensed Matter Physics by Professor Minghu Fang's re-search group. The samples were prepared by a solid-state reaction method from stoichiometric amounts of high purity $\mathrm{La}_{2} \mathrm{O}_{3}, \mathrm{~S}$, Se, and Fe powders. The appropriate mixture of these powders was grounded properly, pelletized, and then heated in an evacuated quartz tube at $1030^{\circ}$ 
C for 72 hours, and the process was repeated three times. Samples obtained were confirmed by x-ray powder diffraction measurements to be of a high-quality single phase $[9,96]$.

\subsubsection{Neutron Powder Diffraction}

Roughly $5 \mathrm{~g}$ of powdered sample was needed to fill a $3 \mathrm{~mm}$ diameter vanadium sample holder. A time-of-flight (T.O.F.) neutron powder diffraction (NPD) measurement was performed at POWGEN diffractometer at the Spallation Neutron Source (SNS) of the Oak Ridge National Laboratory (ORNL). The center wavelengths and $d$-spacing range of neutrons were $1.5 \AA$ and $(0.49664$ - $13.50203 \AA)$ respectively. The data were analyzed using Rietveld refinement were performed using GSAS-II software [85].

\subsubsection{Pair Distribution Function}

We performed a neutron total scattering experiments using the Nano-scale Ordered Materials Diffractometer (NOMAD) beamline at SNS, ORNL. The measurements allowed the determination of the atomic pair distribution function. Both materials $\mathrm{La}_{2} \mathrm{O}_{2} \mathrm{Fe}_{2} \mathrm{O}(\mathrm{S}, \mathrm{Se})_{2}$ powders were loaded and sealed in a vanadium can inside a glove-box. Samples were placed in an orange cryostat and exposed to the neutron beam at various temperatures from $30 \mathrm{~K}$ to $300 \mathrm{~K}$. Sixteen measurements were performed for each sample with additional data points near the Néel temperature. Data were collected for approximately 2 hours at each temperature. An empty $3 \mathrm{~mm}$ vanadium can was also measured for 2 hours in order to collect a background measurement. The background measurement was used to perform background subtraction from the measured samples data.

The atomic pair distribution function $(\mathrm{PDF})$, which involves the Fourier transformation of the total scattering data into real space to obtain the pair-wise atomic 
correlation [67], was calculated by:

$$
\begin{array}{r}
g(r)=1+\frac{1}{2 \pi^{2} r \rho} \int_{Q_{\min }}^{Q \max } Q[S(Q)-1] \sin (Q r) d Q \\
\quad \text { and } G(r)=4 \pi r \rho_{0}(g(r)-1)
\end{array}
$$

where $G(r)$ is the reduced pair distribution function, $S(Q)$ is the normalized structure factor, and $Q$ is the scattering vector. The scattering vector is defined as $Q=\frac{4 \pi}{\sin (\theta)}$, in which $\lambda$ and $\theta$ are the neutron wavelength and scattering angle respectively. The calculated reduced pair distribution function $G_{c}(r)$ is obtained from the structural model using equation (35) [70].

$$
G_{c}(r)=\frac{1}{r} \sum_{i} \sum_{j}\left[\left(\frac{b_{i} b_{j}}{<b>^{2}}\right) \delta\left(r-r_{i j}\right)\right]-4 \pi r \rho_{0}
$$

where $b_{i}$ is,$b_{j}$ and $<b>$ are the average scattering power of the sample atoms. The summation is over all atoms $i$ and $j$ in the model, and $r_{i j}$ is the distance separating any given pair of atoms. PDF data were refined and analyzed using PDFGUI [71] programsuite for the PDF analysis values for $Q_{\min }$ and $Q_{\max }$ were $0.1 \AA$ and 31.4 $\AA$ respectively.

\subsection{Results}

We have performed a neutron powder diffraction at POWGEN at SNS to confirm the average crystal structure. The NPD data were used to perform Rietveld refinement analysis using GSAS-II software [85]. Figure 39 (a) and (b) show the Rietveld refinement of neutron powder diffraction data measured at $300 \mathrm{~K}$ using neutrons of wavelength $1.5 \AA . \mathrm{La}_{2} \mathrm{O}_{2} \mathrm{Fe}_{2} \mathrm{O}(\mathrm{S}, \mathrm{Se})_{2}$ showed nuclear structures that are consistent with a tetragonal system and space group $I 4 / \mathrm{mmm}$, in agreement with the previous studies in literature $[96,9]$. The Rietveld findings show that the lattice parameter and unit cell volume of $\mathrm{La}_{2} \mathrm{O}_{2} \mathrm{Fe}_{2} \mathrm{OSe}_{2}(a=4.08875(2) \AA, c=18.6081(1) \AA$, unit cell 
volume $\left.=311.088(4) \AA^{3}\right)$ are larger than that of the $\mathrm{La}_{2} \mathrm{O}_{2} \mathrm{Fe}_{2} \mathrm{OS}_{2}(a=4.04388(3) \AA$, $c=17.89412(19) \AA$, unit cell volume $\left.=292.622(5) \AA^{3}\right)$ similar to results in reference [9]. Refinement fit results of both $M=\mathrm{S}$ and Se are presented in Tables 10 and 11, respectively. The lattice parameters ( $a$ and $c$ ), the anisotropic thermal parameter $U_{x x}$, atomic site occupancy, and atomic coordination are presented in those tables. The $\mathrm{La}_{2} \mathrm{O}_{2} \mathrm{Fe}_{2} \mathrm{O}(\mathrm{S}, \mathrm{Se})_{2}$ fit quality were characterized by the Rietveld fit parameters $R_{w}$ whole pattern, crystallographic factor $R_{F}$, the goodness of fit GoF, and $\chi^{2}$ which are presented at the bottom of Tables 10 and 11 .

For both materials, The $U_{11}$ value was found to be equal to $U_{22}$ and differs $U_{33}$ from for all atoms except the Fe atom. The $\mathrm{O} 2$ atom had the highest $U_{33}$ value for both material and the Fe atom had different values for each $U_{x x}$ parameter.

The high value of anisotropic thermal displacement parameter $U_{33}$ along the $c$ axis for oxygen $\mathrm{O} 2$, corresponded to the displacement of $\mathrm{O} 2$ atom above and below the $\mathrm{Fe}_{2} \mathrm{O}$ plane which can result in a distortion of Fe-O-Fe angle (in Figure 37, the Fe-O-Fe angle is highlighted by a solid black line in the octahedra). Another possible movement within the octahedra, is the movement of the sulfur/selenium atoms with respect to the Fe atoms along the $c$-axis. This motion can affect the $M$-Fe- $M$ angle in the octahedra. Figure 38 shows $\mathrm{Fe}$ and $\mathrm{O} 2$ atomic movements in the octahedra. In panel a, the atoms are viewed along the $a$-axis, and in panel $\mathrm{b}$, the view is slightly rotated to give an isometric view of panel a. When the $\mathrm{O} 2$ atoms in the $\mathrm{Fe}_{2} \mathrm{O}$ plane move up or down, the angle Fe-O-Fe changes.

After confirming the average structure lattice symmetry for $\mathrm{La}_{2} \mathrm{O}_{2} \mathrm{Fe}_{2} \mathrm{O}(\mathrm{S}, \mathrm{Se})_{2}$ samples, we investigated the local symmetry by the pair distribution function (PDF) method. The PDF method has been shown to be an effective method for studying local crystal structure nematic fluctuations [100, 101, 102, 103, 104, 105]. Studying the local structure of $\mathrm{La}_{2} \mathrm{O}_{2} \mathrm{Fe}_{2} \mathrm{O} M_{2}(M=\mathrm{S}$, Se) via PDF refinement method to determine the localized structural arrangement as a function of temperature and length 
a)
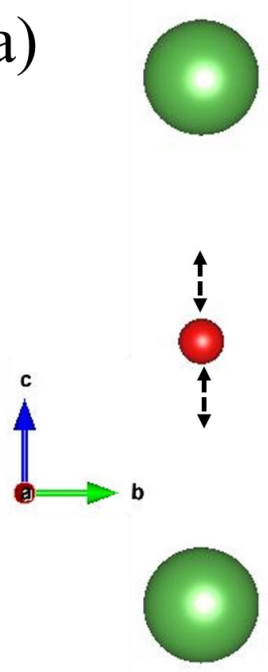
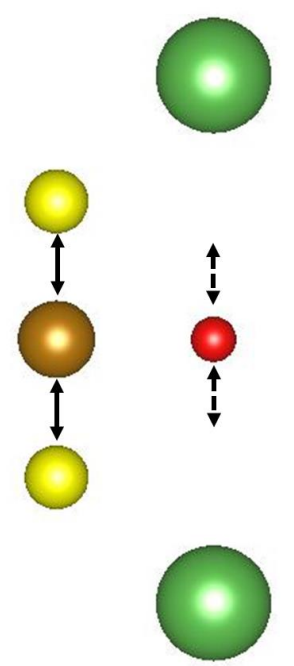

b)
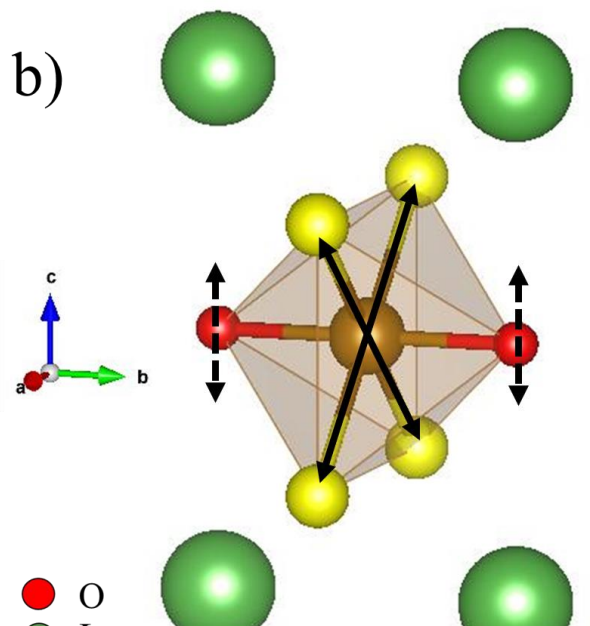

$\mathrm{La}$

$\mathrm{Fe}$

$M=\mathrm{S}, \mathrm{Se}$

Figure 38. Panel a) shows possible $\mathrm{Fe}$ and the chalcogen atomic movement within the $\mathrm{La}_{2} \mathrm{O}_{2} \mathrm{Fe}_{2} \mathrm{O}(\mathrm{S}, \mathrm{Se})_{2}$ octahedra. The dashed lines in b) show the $\mathrm{O} 2$ atom movement in the $\mathrm{Fe}_{2} \mathrm{O}$ plane. The solid lines show Fe atom movement with respect to the chalcogen atom $M[16]$.

scale was done using the PDFGUI program [71]. All lattice parameters, scale factor, atomic positions, and the thermal parameters were refined during the PDF analysis process. To make the refinement robust, we parametrized the lattice constants as $a=a_{m i d}(1+\delta)$ and $b=a_{m i d}(1-\delta)$, where $\mathrm{a}_{\text {mid }}=\frac{a+b}{2}$ and $\delta=\left|\frac{a-b}{a+b}\right|$. We have used Nyquist grid to perform all refinements. Instrumental parameters $q_{d a m p}$ and $q_{b r o a d}$, which are the parameters that correct the PDF envelope function $[106,107]$ for the instrument resolution [70, 71], were fixed.

PDFGUI, through pre-written macros, allows data set fitting through a series of refinements that differ, one from another, by the corresponding fitting ranges [108]. Since we have performed PDF data sets over a wide range of temperatures, we used the PDFGUI $T$-series macro to study the details of the local to average structure crossover in our materials. This macros enable the set up of $T$-series sequential refinement fits where the same model is fitted over different temperatures of the PDF 

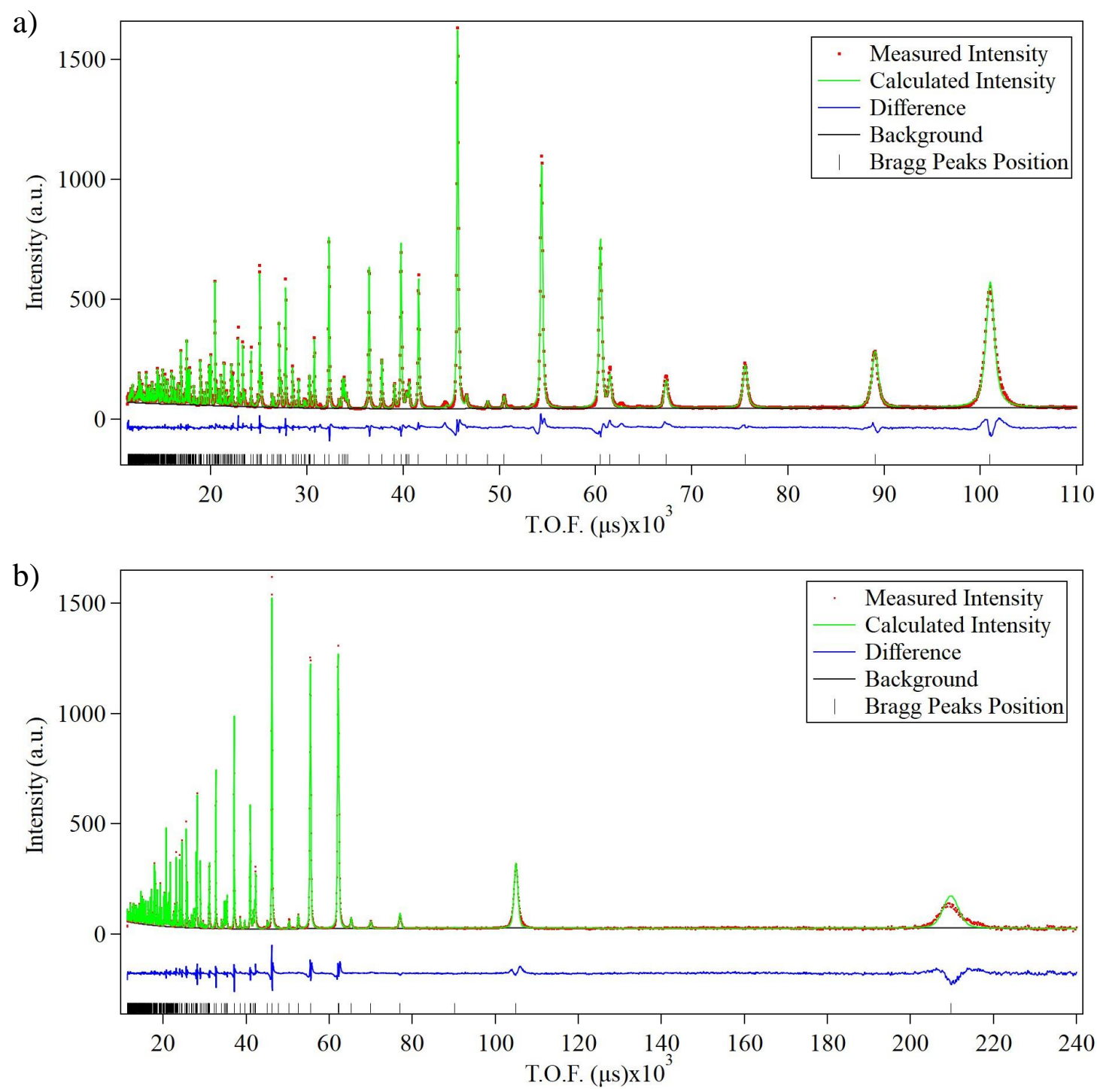

Figure 39. Nuclear structure Rietveld refinement profile of NPD of a) $M=\mathrm{S}$ and b) $M=$ Se at $300 \mathrm{~K}$. 
Table 10. Rietveld Refinement results for $\mathrm{La}_{2} \mathrm{O}_{2} \mathrm{Fe}_{2} \mathrm{OSe}_{2}$ from room temperature neutron powder diffraction. Space Group I4/mmm $a=4.08875(2) \AA, c=18.6081(1)$ $\AA$ and unit cell volume $=311.088(4) \AA^{3}$.

\begin{tabular}{lccccccc}
\hline \multicolumn{7}{c}{$\mathrm{La}_{2} \mathrm{O}_{2} \mathrm{Fe}_{2} \mathrm{OSe}_{2}$} \\
\hline Atom & $\mathrm{x}$ & $\mathrm{y}$ & $\mathrm{z}$ & Occup. & $\mathrm{U}_{11}\left(\AA^{2}\right)$ & $\mathrm{U}_{22}\left(\AA^{2}\right)$ & $\mathrm{U}_{33}\left(\AA^{2}\right)$ \\
$\mathrm{La}$ & 0.5 & 0.5 & $0.18450(6)$ & $0.972(5)$ & $0.0029(3)$ & $0.0029(3)$ & $0.0076(6)$ \\
$\mathrm{O} 1$ & 0.5 & 0.0 & 0.25 & $0.996(6)$ & $0.0040(4)$ & $0.0040(4)$ & $0.0112(8)$ \\
$\mathrm{O} 2$ & 0.5 & 0.5 & 0.0 & $0.970(11)$ & $0.0055(6)$ & $0.0055(6)$ & $0.0218(14)$ \\
$\mathrm{Fe}$ & 0.5 & 0.0 & 0.0 & $0.936(5)$ & $0.0065(4)$ & $0.0052(4)$ & $0.0106(5)$ \\
$\mathrm{Se}$ & 0.0 & 0.0 & $0.9666(6)$ & $0.950(5)$ & $0.0064(3)$ & $0.0064(3)$ & $0.0041(5)$
\end{tabular}

Coordination

$\begin{array}{lc}d_{F e-O 1}(\AA) & 4.6520(0) \\ d_{F e-O 2}(\AA) & 2.0444(0) \\ d_{F e-S e}(\AA) & 2.723(8) \\ d_{F e-F e}(\AA) & 2.8912(0) \\ d_{L a-O 1}(\AA) & 2.3801(6) \\ d_{L a-S e}(\AA) & 3.3212(8) \\ a_{F e-O 2-F e} \text { (deg.) } & 90.0(0) \\ a_{F e-S e_{F} e} \text { (deg.) } & 64.13(2) \\ a_{S e-F e_{S} e} \text { (deg.) } & 97.32(4) \\ a_{L a-O 1-L a} \text { (deg.) } & 118.39(5)\end{array}$

$\mathrm{wR}_{P}=0.0675 \quad \mathrm{R}_{P}=0.0647 \quad \mathrm{GoF}=5.35 \quad \chi^{2}=0.2836$

data [109]. We have performed extensive $T$-series PDF fits to determine the atomic structure as a function of temperature and length scale [101]. The evolution of the local structure (short-range) to the average structure (long-range) is presented in Figure 40. The most relevant structural parameter for describing the local distortion is the orthorhombicity. The orthorhombicity was determined, for both $M=\mathrm{S}$ and Se, by using the results of $T$-series refinements based on the orthorhombic symmetry. $T$-series refinement was performed by PDF fits on low-temperature data first, a sequential PDF fits were performed for all of our data collected at various temperatures. Both $M=\mathrm{S}$ and Se show high orthorhombicity in the short ranges for all temperatures at which data are collected. 
Table 11. Rietveld Refinement results for from neutron powder diffraction at $300 \mathrm{~K}$. Space Group $I 4 / m m m a=4.04388(3) \AA, c=17.89412(19) \AA$ and unit cell volume $=292.622(5) \AA^{3}$.

\begin{tabular}{lccccccc}
\hline \multicolumn{7}{c}{$\mathrm{La}_{2} \mathrm{O}_{2} \mathrm{Fe}_{2} \mathrm{OS}_{2}$} \\
\hline Atom & $\mathrm{x}$ & $\mathrm{y}$ & $\mathrm{z}$ & Occup. & $\mathrm{U}_{11}\left(\AA^{2}\right)$ & $\mathrm{U}_{22}\left(\AA^{2}\right)$ & $\mathrm{U}_{33}\left(\AA^{2}\right)$ \\
$\mathrm{La}$ & 0.5 & 0.5 & $0.18082(5)$ & $0.898(4)$ & $0.0016(2)$ & $0.0016(2)$ & $0.0033(4)$ \\
$\mathrm{O} 1$ & 0.5 & 0.0 & 0.25 & $0.897(4)$ & $0.0007(3)$ & $0.0007(3)$ & $0.0062(6)$ \\
$\mathrm{O} 2$ & 0.5 & 0.5 & 0.0 & $0.874(7)$ & $0.0002(4)$ & $0.0002(4)$ & $0.0281(14)$ \\
$\mathrm{Fe}$ & 0.5 & 0.0 & 0.0 & $0.871(4)$ & $0.0082(4)$ & $0.0020(3)$ & $0.0112(5)$ \\
$\mathrm{S}$ & 0.0 & 0.0 & $0.9397(15)$ & $0.857(5)$ & $0.0054(6)$ & $0.0054(6)$ & $0.0007(3)$
\end{tabular}

Coordination

$$
\begin{aligned}
& d_{F e-O 1}(\AA) \quad 5.30933(0) \\
& d_{F e-O 2}(\AA) \quad 2.02194(0) \\
& d_{F e-S}(\AA) \quad 2.6298(18) \\
& d_{F e-F e}(\AA) \quad 2.85946(0) \\
& d_{L a-O 1}(\AA) \quad 2.3708(5) \\
& d_{L a-S}(\AA) \quad 3.2545(14) \\
& a_{\mathrm{Fe}-\mathrm{O} 2-\mathrm{Fe}} \text { (deg.) } \quad 90.0(0) \\
& a_{F e-S_{F} e} \text { (deg.) } \quad 65.87(5) \\
& a_{S-F e_{S}} \text { (deg.) } \quad 100.50(9) \\
& a_{L a-O 1-L a} \text { (deg.) } 117.05(4) \\
& \mathrm{wR}_{P}=0.0501 \quad \mathrm{R}_{P}=0.0561 \quad \mathrm{GoF}=4.86 \quad \chi^{2}=0.2336
\end{aligned}
$$



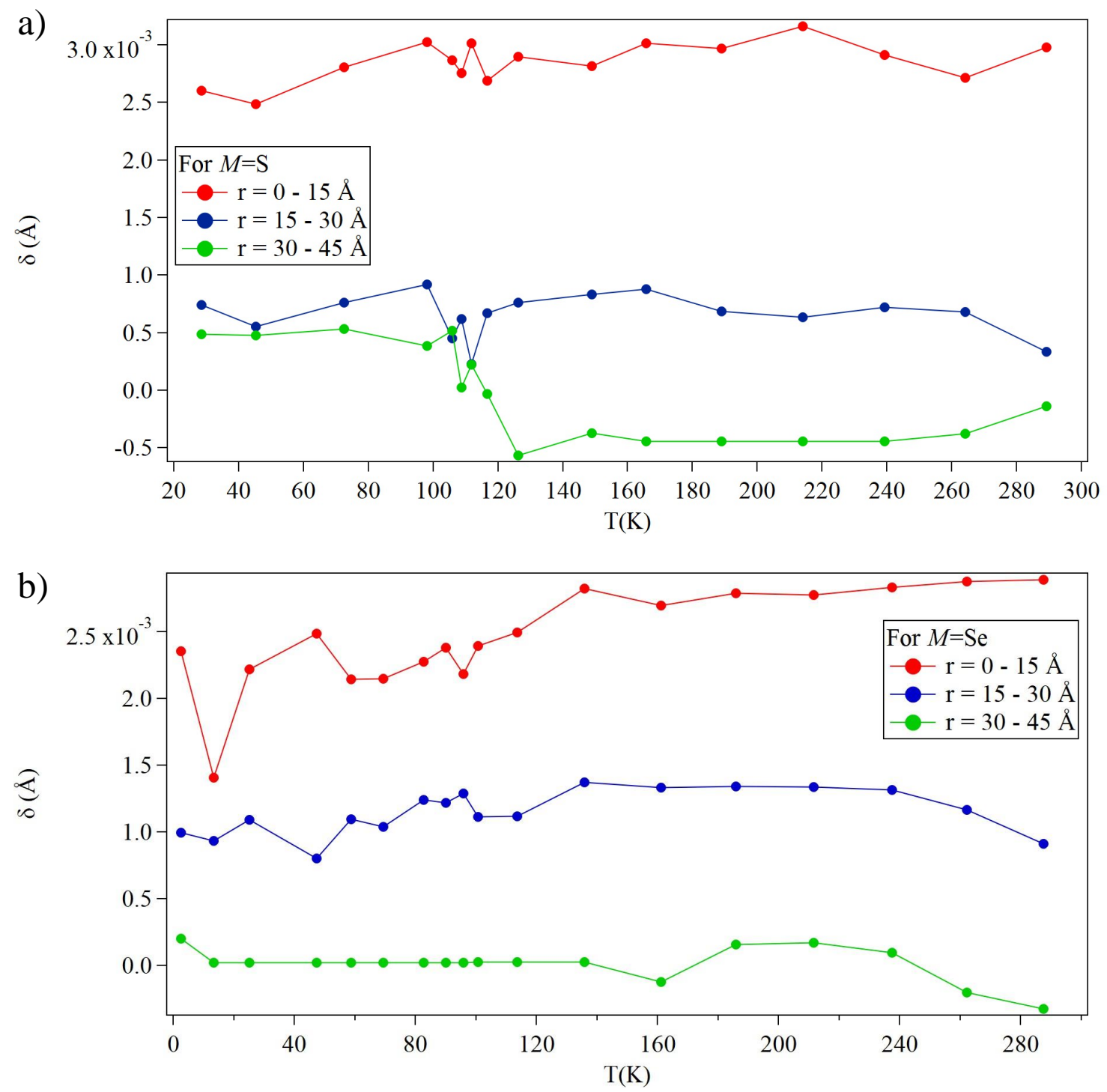

Figure 40. The orthorhombicity determined from neutron PDF analysis for a) $M=$ $\mathrm{S}$ and b) $M=$ Se. At the short ranges over all temperatures, we observe the presence of orthorhombicity for both samples. 


\subsection{Discussions}

Neutron powder diffraction experiments established the average crystal structure as a tetragonal system with space group $(I 4 / \mathrm{mmm})$ for both $\mathrm{La}_{2} \mathrm{O}_{2} \mathrm{Fe}_{2} \mathrm{O}(\mathrm{S}, \mathrm{Se})_{2}$. These findings match those observed in earlier studies on iron oxychalcogenides [96, 95, 98, 99, 13, 9]. Results from the neutron powder diffraction (Figure 39, Tables 11 and 10) clearly show that the thermal displacement parameter for $\mathrm{O} 2$ atom, along the $c$-axis $\left(U_{33}\right)$, is larger than all the other thermal displacement parameters for both materials $M=\mathrm{S}$ and Se.

According to Horigane et al. [110], deviations in the lattice parameter from the average crystallographic structure are caused by thermal displacement parameter $U_{33}$ for $\mathrm{O} 2$ along the $c$-axis. It was claimed that this might be a similar case as of $\mathrm{SrFeO}_{2}$ [111] which has a stronger temperature dependence of $U_{33}$ for $\mathrm{O} 2$ (in-plane). In $\mathrm{La}_{2} \mathrm{O}_{2} \mathrm{Fe}_{2} \mathrm{OSe}_{2}$, the high $U_{33}$ for $\mathrm{O} 2$ suggests the possibility of large distortion in $\mathrm{O} 2$ site. Also, a distortion in the Fe site makes it possible to have two Fe-Se correlation. In $\mathrm{La}_{2} \mathrm{O}_{2} \mathrm{Fe}_{2} \mathrm{OSe}_{2}$, the high $U_{33}$ for $\mathrm{O} 2$ suggests the possibility of large distortion in $\mathrm{O} 2$ site. Also, a distortion in the Fe site makes it possible to have two Fe-Se correlation.

Figure 41 compares the phase diagram of iron pnictides (Figure 41 a) and $\mathrm{La}_{2} \mathrm{O}_{2} \mathrm{Fe}_{2} \mathrm{O}(\mathrm{S}$, Se) ${ }_{2}$ Mott insulators (Figure $41 \mathrm{~b}$ and c). In the phase diagram of FeSCs (Figure $41 \mathrm{a}$ ), the normal state is shown in light green color on the right/top part of the phase diagram. In this state, the FeSC is in the tetragonal crystal structure and paramagnetic magnetic phase at high temperatures. At lower temperature, the doping affects the behavior of the compound, it shows nematic fluctuation and ordering at $T_{\text {nem. }}$, a structural phase transition to the orthorhombic structure at $T_{c}$ and magnetic phase transition to the antiferromagnetic structure at $T_{N}$ as well as superconductivity properties that emerge at certain dopings.

In the pure sulfur and selenium coumpounds of $\mathrm{La}_{2} \mathrm{O}_{2} \mathrm{Fe}_{2} \mathrm{O}(\mathrm{S}, \mathrm{Se})_{2}$ we have studied, we found evidence of nematicity at the shorter length scales (Figure 40 red lines 
for $r=0-15 \AA$ ), which is shown in the phase diagram (Figure $41 \mathrm{~b}$ and c). Unlike iron pnictides, which undergo a structural phase transition from tetragonal to orthorhombic around $T_{s}, \mathrm{La}_{2} \mathrm{O}_{2} \mathrm{Fe}_{2} \mathrm{O}(\mathrm{S}, \mathrm{Se})_{2}$ lattice symmetry does not change with temperature. Similar to iron pnictide superconductors, both $\mathrm{La}_{2} \mathrm{O}_{2} \mathrm{Fe}_{2} \mathrm{O}(\mathrm{S}, \mathrm{Se})_{2}$ undergo a magnetic phase transition at Néel temperature $T_{N}$ from a paramagnetic (PM) phase to an antiferromagnetic (AFM) phase. Nematic fluctuations appear around $T_{s}$ in superconducting iron pnictides. These nematic fluctuations persist to a more ordered phase (nematic phase) until the temperature reaches the Néel temperature at which the magnetic phase transition occurs.

The superconductivity, in most FeSCs, is found in the proximity to a magnetically ordered state. However, a careful study of the phase diagram reveals that at a specific temperature of $T_{n e m}$, there is another non-superconducting state besides magnetism. In this state, the system breaks its symmetry between $\mathrm{x}$ and $\mathrm{y}$ directions in the Feplane [17]. This system break reduces the rotational point group symmetry from tetragonal to orthorhombic while preserving the translational symmetry, also known as nematic fluctuations [101, 112].

The superconductivity, in most FeSCs, is found in the proximity to a magnetically ordered state. However, a careful study of the phase diagram reveals that at a specific temperature of $T_{n e m}$, there is another non-superconducting state besides magnetism. In this state, the system breaks its symmetry between $\mathrm{x}$ and $\mathrm{y}$ directions in the Feplane [17]. This system break reduces the rotational point group symmetry from tetragonal to orthorhombic while preserving the translational symmetry, also known as nematic fluctuations $[101,112]$.

In FeSCs and other related materials, vital information can be understood not only from the nematic phase, which occurs above $T_{s}$ but also from the nematic fluctuations which occur over a broad range of temperatures. These fluctuations, which deviates from the average structure, provide important information about the origin 

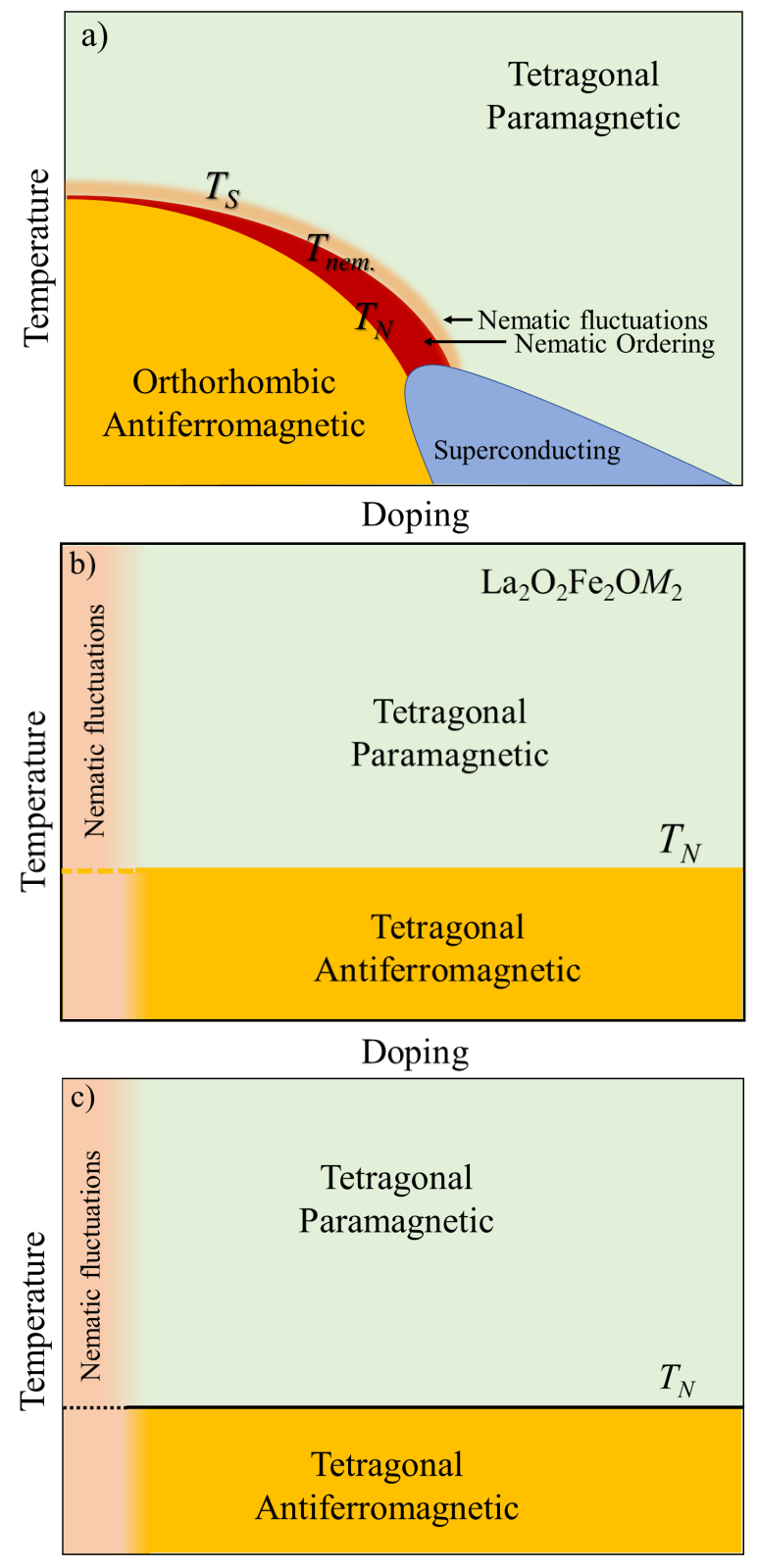

Length Scale

Figure 41. A comparison of phase diagram in iron pnictides superconductors and the preliminary phase diagram of $\mathrm{La}_{2} \mathrm{O}_{2} \mathrm{Fe}_{2} \mathrm{O}(\mathrm{S}, \mathrm{Se})_{2}$ Mott insulators. Panel a) after References [17, 18, 19, 20].

of nematicity and its relation to superconductivity mechanism $[113,114,115,116$, $100,117,118,119]$. This question has become the key focal point of the field of iron-based high- $T_{c}$ superconductivity. The findings presented in current study and in literature suggest that nematic fluctuations are not exclusively required for the onset 
of a superconducting phase. In iron pnictides and iron chalcogenides, nematic fluctuations, along with other phases, appear to conspired to produce high-temperature superconductivity.

\subsection{Conclusions}

In this study, the average and local structure of the Mott insulating iron oxychalcogenides $\mathrm{La}_{2} \mathrm{O}_{2} \mathrm{Fe}_{2} \mathrm{O}(\mathrm{S}, \mathrm{Se})_{2}$ were studied using neutron powder diffraction and pair distribution techniques. Neutron powder diffraction showed a similar average nuclear structure of $M=$ Se and S, with differences in volume and lattice parameters corresponding to differences in the atomic radii of the two chalcogens $\mathrm{S}$ and Se. Pair distribution function analysis showed the presence of local distortions from tetragonal to orthorhombic symmetry over the short-range, especially at lower temperatures. These results suggest the presence of short-range local distortions in both of these materials. These distortions can be evidence of nematic short-range ordering in the $\mathrm{La}_{2} \mathrm{O}_{2} \mathrm{Fe}_{2} \mathrm{O}(\mathrm{S}, \mathrm{Se})_{2}$ materials despite the persistent tetragonal long-range crystal structure.

\subsection{Future Work}

Local structure investigation in similar materials, iron based superconductors and Mott insulators can be carried out using the PDF analysis to further examine the short range fluctuation in these materials and explore its nematic fluctuation and nematic ordering. Similar PDF analysis will be applied to other iron-based materials, including $\mathrm{Fe}_{1.1} \mathrm{Te}$ to investigate the presence of nematic fluctuations. 


\section{CHAPTER 7}

\section{STRUCTURAL STUDY OF MOLYBDENUM DITELLURIDE $2 \mathrm{H}-\mathrm{MoTe}_{2}$ AND $1 T^{\prime}-\mathrm{MoTe}_{2}$}

\subsection{Introduction}

Transition metal dichalcogenides (TMDs) have a general form $M X_{2}$ where $M=$ Mo, W, Ti, Zr, Hf, V, Nb, Ta, Tc, Re, Co, Rh, Ir, Ni, Pd, Pt) and $X=\mathrm{S}, \mathrm{Se}$, Te. Molybdenum-based TMD materials have received theoretical and experimental attention because of their potential in advancing the development of a new generation of electronics devices beyond silicon and graphene [120, 121, 122]. A typical TMD shows the trigonal prismatic $2 H$ or the octahedral $1 T$ structures [123]. Figure 42 shows trigonal coordination of $\mathrm{MoTe}_{2}$.

Molybdenum ditelluride $\mathrm{MoTe}_{2}$ crystallizes into three stable phases: $2 H, 1 T^{\prime}$ and $T_{d}$ while the $1 T$ is considered to be unstable [123]. Table 12 provides the previously reported lattice parameters, space group and the crystal system of all phase of $\mathrm{MoTe}_{2}$. The $2 H$, called $\alpha-\mathrm{MoTe}_{2}$, is a stable semiconductor with hexagonal structure and $P 6_{3} / m m c$ space group $[124,125]$. The $2 \mathrm{H}-\mathrm{MoTe}_{2}$ does not undergo a structural phase transition with temperature, it may achieve superconductivity with applied pressure [126]. Hexagonal-phase molybdenum ditelluride $\mathrm{MoTe}_{2}$ has been studied for use in photo-electrochemical solar cells [127]. Optoelectronic, spintronic, and valleytronic devices $[128,129]$ are possible applications for $\mathrm{MoTe}_{2}$ due to its small bandgap and large spin-orbit (SO) coupling. $\alpha-\mathrm{MoTe}_{2}$ has also been proposed as a base material for $p n$-junctions [130, 131], field-effect transistors [131], complementary 
a)

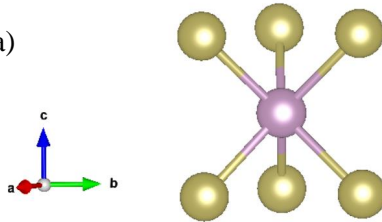

b)

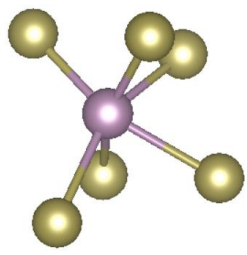

c)

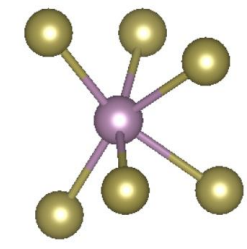

Figure 42. Prismatic coordination of $\mathrm{MoTe}_{2}$. Panel a), b) and c) shows the coordination for $2 \mathrm{H}-\mathrm{MoTe}_{2}, 1 T^{\prime}-\mathrm{MoTe}_{2}$, and $T_{d}-\mathrm{MoTe}_{2}$ phase.

metal-oxide-semiconductor (CMOS) devices [132, 133], Schottky barriers [134] and gas sensors [135].

Table 12. Schematics of the structural polytypes of $\mathrm{MoTe}_{2}$.

\begin{tabular}{ccccccc}
\hline Phases & $a(\AA)$ & $b(\AA)$ & $c(\AA)$ & Space Group & Crystal System & Reference \\
\hline $1 T-\mathrm{MoTe}_{2}$ & - & - & - & Unstable & - & {$[123]$} \\
$1 T_{d^{-}-\mathrm{MoTe}_{2}}$ & 3.471 & 6.338 & 13.858 & $P m n 2_{1}($ no.31) & Orthorhombic & {$[136]$} \\
$1 T^{\prime}-\mathrm{MoTe}_{2}$ & 6.338 & 3.472 & 13.861 & $P 2_{1} / m($ no.11) & Monoclinic & {$[137]$} \\
$2 H-\mathrm{MoTe}_{2}$ & 3.519 & 3.519 & 13.964 & $P 6_{3} / m m c($ no.194) & Hexagonal & {$[124]$} \\
\hline
\end{tabular}

The $\beta-\mathrm{MoTe}_{2}$ (1T phase) has distorted octahedra showing monoclinic structure with space group $P 2_{1} / m[137,138]$. $1 T^{\prime}-\mathrm{MoTe}_{2}$ undergoes through structural phase transition with temperature to the $T_{d}-\mathrm{MoTe}_{2}$ phase. The $T_{d}-\mathrm{MoTe}_{2}$ version has attracted attention because it exhibited superconductivity at $T_{c}=0.1 \mathrm{~K}$ and a nontrivial $\pi$ Berry phase at low temperature. $T_{d}-\mathrm{MoTe}_{2}$ is considered to be the first type-II Weyl semimetal (WSM) $[4,139]$ and is predicted to be a topological superconductor $[140,141] . T_{d}-\mathrm{MoTe}_{2}$ is a WSM type-II which characterized by point-like Fermi 
surface. Weyl fermions in $T_{d}-\mathrm{MoTe}_{2}$ are very sensitive to their lattice constant. This provides a way to tune the topological phase transition by pressure or temperature [136].

The $2 \mathrm{H}-\mathrm{MoTe}_{2}$ phase consists of one layer of transition metal atoms sandwiched by two layers of chalcogen atoms with a prismatic coordination. The molybdenum Mo atom is surrounded by uniformly displaced 6 tellurium atoms Te arranged at the vertices of a triangular prism as shown in Figure 42, a. In $1 T^{\prime}-\mathrm{MoTe}_{2}$ and $T_{d^{-}}$ $\mathrm{MoTe}_{2}$ phases the prism is distorted octahedrally coordinated by Te with alternating long and short distances between Mo atoms due to strong intermetallic bonding. Figure $42 \mathrm{~b}$ and c shows the Prismatic coordination of $1 T^{\prime}-\mathrm{MoTe}_{2}$ and $T_{d^{-}} \mathrm{MoTe}_{2}$ respectively. Both structure contain bonds between the metal atoms that dimerize along one of the lattice directions $[142,143]$.

There is considerable interest in the phase transition behavior of $\mathrm{MoTe}_{2}$ because the phase changes are often coupled with other changes in these low-dimensional systems $[144,145]$. The structural phase transition from $2 H$ to $1 T^{\prime}$ phase in a few-layer $\mathrm{MoTe}_{2}$ was found to be driven by electrostatic doping [146] and by laser irradiation [147]. Structural phase transitions from the $T_{d}$ to $1 T^{\prime}$ phase, can be driven by temperature [148, 149], are accompanied by topological phase transition.

Trigonal prismatic (see figure 42 a) $\alpha$-MoTe 2 form is stabilized by the opening of a hybridization gap within the Mo $d$-band manifold, splitting off the full $d_{z^{2}}$ band. However, such a gap does not occur for the ideal octahedral coordinate structure (see figure 42 b) [150, 151]. Due to a trigonal prismatic crystal-field splitting of a filled nonbonding $d_{z^{2}}$ orbital, the group VIb di-tellurides show a deviation from a simple band model, which indicates semiconducting behavior.

Three-dimensional (3D) topological Weyl semimetals (TWSs) [152] are novel topological quantum materials discovered and intensively investigated recently because of their intimate link between concepts of different fields of physics and material science. 
a)

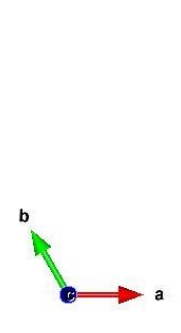

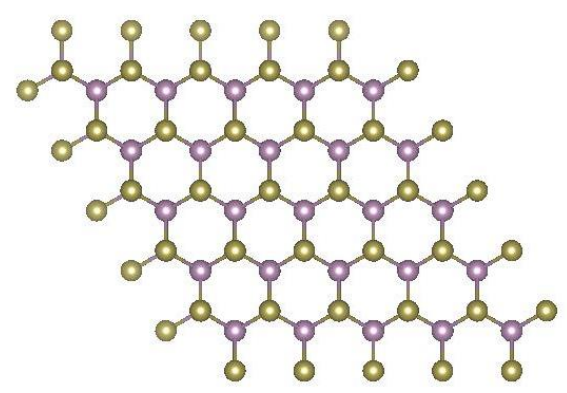

c)
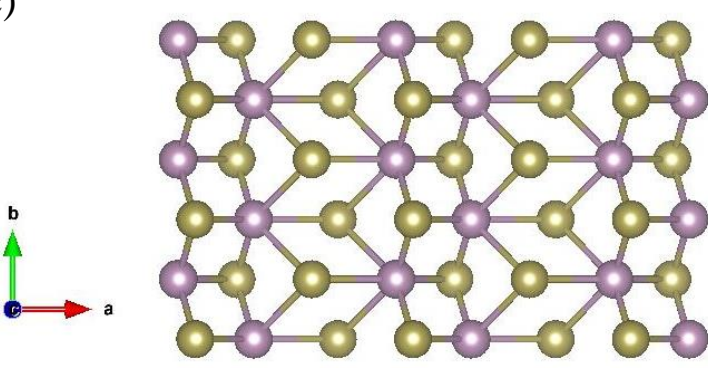

e)

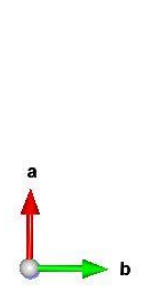

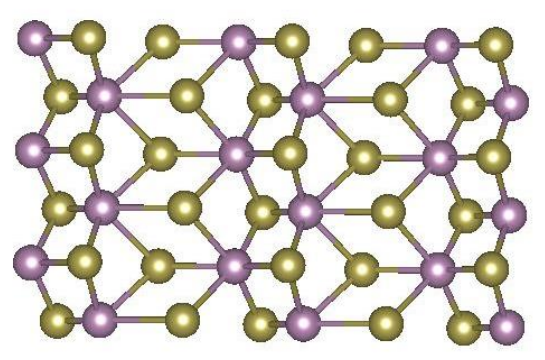

b)

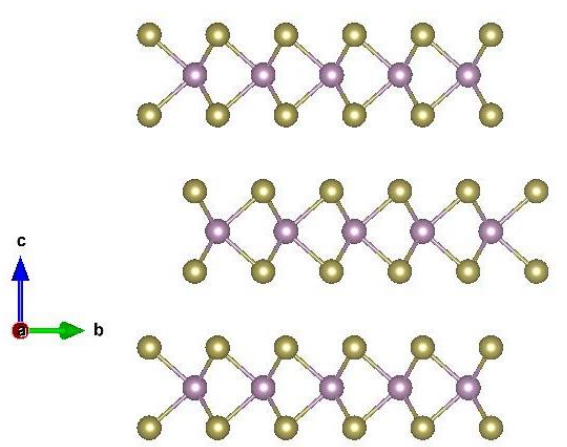

d)

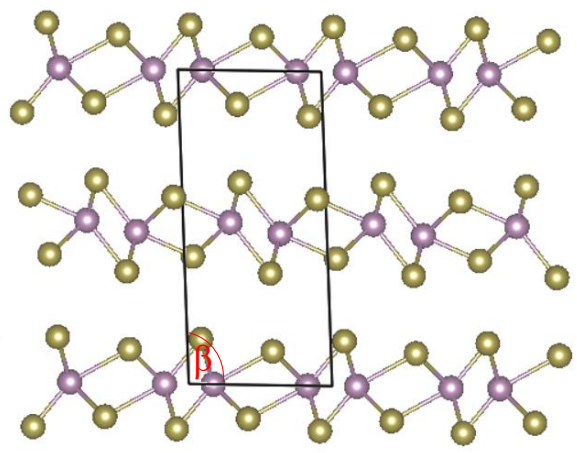

f)

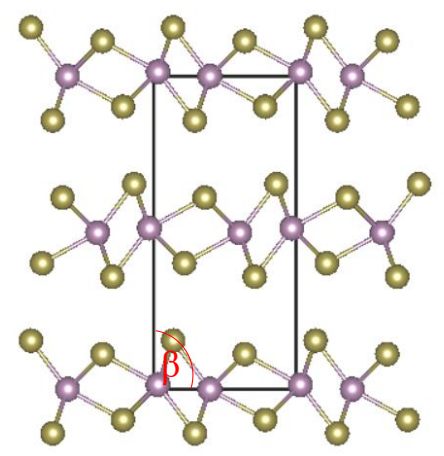

Mo Te

Figure 43. The structure of different phases of $\mathrm{MoTe}_{2}$. Panel a) shows top view of $2 H-\mathrm{MoTe}_{2}$, panel b) side view of $2 \mathrm{H}-\mathrm{MoTe}_{2}$. The top view of $1 T^{\prime}-\mathrm{MoTe}_{2}$ is shown in panel c), whereas panel d) shown the side view of the same phase. The $T_{d}-\mathrm{MoTe}_{2}$ top view is shown in panel e) and the side view on panel $\mathrm{f}$ ).

Topological TWSs display a range of physical properties of promising usage [153]. Theoretical prediction claims that $2 H$ TMDs may become superconductors at high pressure. $\mathrm{MoTe}_{2}$ shows superconductivity at transition temperature $T_{c}=0.1 \mathrm{~K}$. At high pressure, about $11.7 \mathrm{GPa}$, the transition temperature $T_{c}$ goes up to $8.2 \mathrm{~K}[141]$. In the monolayer at low-temperature, $T_{d}-\mathrm{MoTe}_{2}$ phase is a type-II Weyl semimetal. 


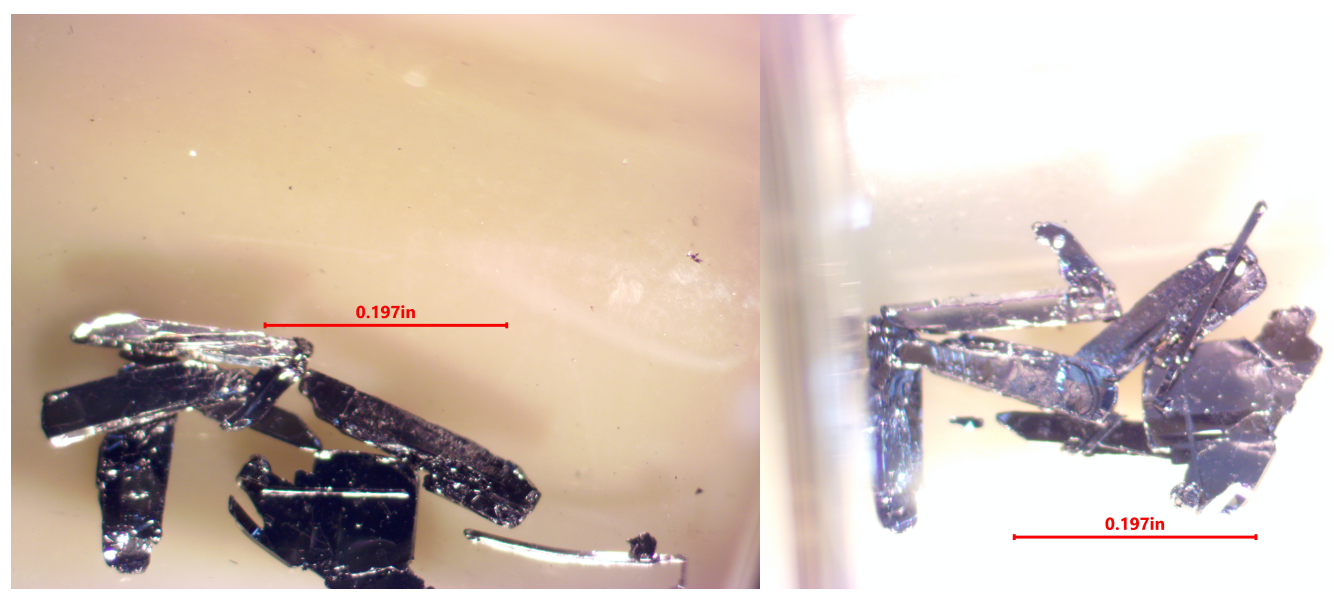

Figure 44. single crystal $1 T^{\prime}-\mathrm{MoTe}_{2}$.

It shows topological surface state Fermi arcs and chiral anomalies. Induced negative magnetoresistance is formed through temperature-driven phase transition from $1 T^{\prime}$ $\mathrm{MoTe}_{2}[154,155]$. During the phase transition from $1 T^{\prime}-\mathrm{MoTe}_{2}$ to $T_{d}-\mathrm{MoTe}_{2}$ where the electronic structure is largely preserved, but the topological character is changed. Using physical knobs such as strain or pressure at low temperatures the topological phenomena can be turned on and off by small changes in temperature. Where the structural transition also acts as a topological switch for surface states in $1 T^{\prime}$ $\mathrm{MoTe}_{2}[140]$.

Currently, there is ambiguity concerning the nature of the phase transition in $1 T^{\prime}-\mathrm{MoTe}_{2}$. There are less than a handful of high-resolution structural studies at low-temperature. We sought to examine the phase transitions of $2 \mathrm{H}-\mathrm{MoTe}_{2}$ and $1 T^{\prime}-\mathrm{MoTe}_{2}$. We used neutron powder diffraction and high-resolution x-ray powder diffraction to investigate both phases. Understanding the structure of $\mathrm{MoTe}_{2}$ in fewlayer and bulk configurations is important for enhancing our ability to deploy the material as topological devices. 


\subsection{Material}

\subsubsection{Synthesis}

Samples used in the present experiments were obtained from two sources: commercial samples from HQ graphene and from the University of Tennessee. Hq graphene $2 H-\mathrm{MoTe}_{2}$ and $1 T^{\prime}-\mathrm{MoTe}_{2}$ crystals were grown by the chemical vapor transport method. Figure 44 shows a picture of $1 T^{\prime}-\mathrm{MoTe}_{2}$ crystal obtained from hq graphene and the size of the longest edge of some crystals is about $5 \mathrm{~mm}$ (0.197 in).

$\mathrm{MoTe}_{2}$ samples were grown by the Yan group in the Materials Science and Engineering Department at the University of Tennessee at Knoxville. $1 T^{\prime}-\mathrm{MoTe}_{2}$ single crystals were grown out of a Te-rich binary melt using a canfield crucible set (CCS). Mo and Te shots in a ratio of 1:9 were loaded into a $5 \mathrm{ml}$ CCS and sealed in a quartz tube under vacuum. The quartz ampoule was held at $1000^{\circ} \mathrm{C}$ for a week. $1 T^{\prime}-\mathrm{MoTe}_{2}$ single crystals were isolated from Te flux by centrifuging. We have sin-

gle crystalline samples of $1 T^{\prime}-\mathrm{MoTe}_{2}$. Single crystals grown in this manner have a residual resistance ration (RRR) 500 and magnetoresistance (MR) of 40,000 which indicated no (or low) amounts of impurities and crystal defects. Samples sizes were plate-like with typical dimensions $2 \mathrm{~mm}$ x $4 \mathrm{~mm}$ x $0.3 \mathrm{~mm}$.

\subsubsection{Characterization}

\subsubsection{Raman Spectroscopy}

$2 \mathrm{H}-\mathrm{MoTe}_{2}$ and $1 T^{\prime}-\mathrm{MoTe}_{2}$ samples were measured using a commercial Horiba LabRam HR Raman microscope in the back-scattering geometry at room temperature in Prof. Lui lab at University of California Riverside. The excitation light source was a $532 \mathrm{~nm}$ continuous laser. The incident laser power on the samples was below 1 $\mathrm{mW}$, with a spot diameter of $1 \mu \mathrm{m}$ [156]. The present study performed detailed crystal structure analysis on $\mathrm{MoTe}_{2}$ material by studying the structural changes of the 

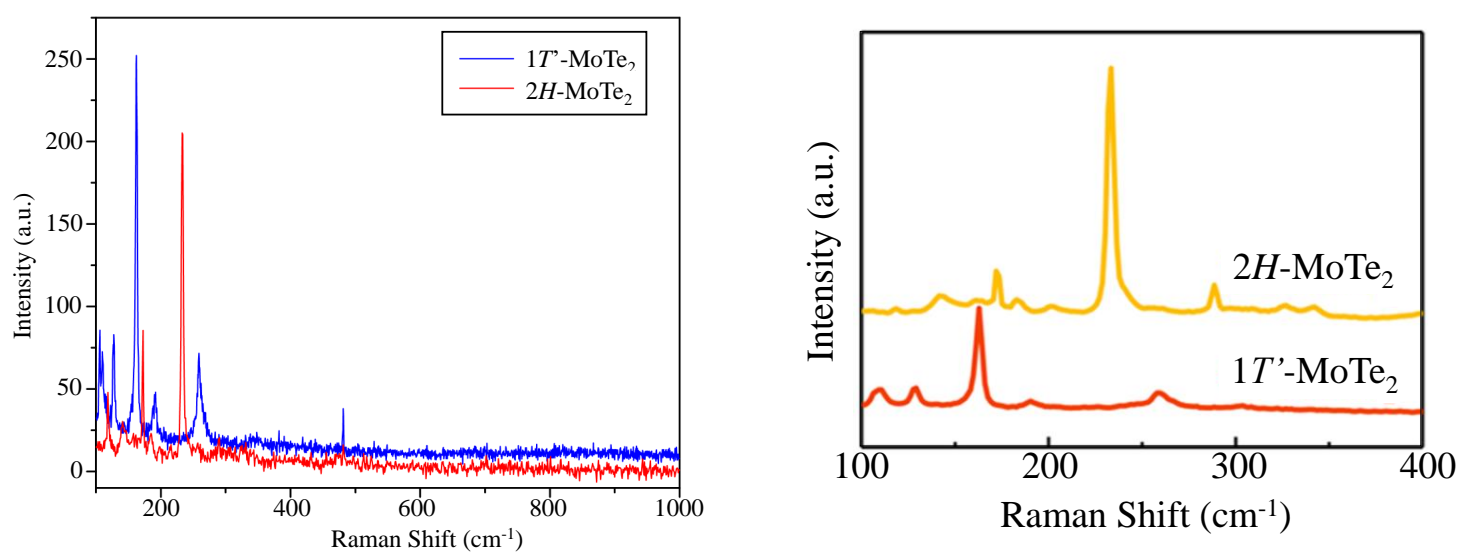

Figure 45. Left panel shows Raman Measurement on powder sample of $2 \mathrm{H}-\mathrm{MoTe}_{2}$ and $1 T^{\prime}-\mathrm{MoTe}_{2}$. Right panel shows the Raman spectra for both samples from reference [21].

$2 H-\mathrm{MoTe}_{2}$ and $1 T^{\prime}-\mathrm{MoTe}_{2}$ phases with respect to temperature. To confirm the quality of both samples, $2 \mathrm{H}-\mathrm{MoTe}_{2}$ and $1 T^{\prime}-\mathrm{MoTe}_{2}$, we performed Raman spectroscopy. Raman measurements have been used previously to identify the type of material. Our Raman measurements were intended to determine weather the samples did not include contaminants. Also, Raman spectroscopy would have shown the different phases present in the material. The Raman spectroscopy results (Figure 45) confirm that only one phase exists in each sample which is essential for accurate study of the temperature effects on the structure of each phase. The Raman results also showed that both samples were clear from impurities. These results are similar to what is reported in the literature for both samples of $2 H-\mathrm{MoTe}_{2}$ and $1 T^{\prime}-\mathrm{MoTe}_{2}$ [21].

\subsubsection{Electron dispersion x-ray Spectroscopy (EDX)}

$2 \mathrm{H}-\mathrm{MoTe}_{2}$ and $1 T^{\prime}-\mathrm{MoTe}_{2}$ samples were characterized using energy dispersion x-ray spectroscopy (EDX) measurements performed at the University of Louisville, CONN Center for Renewable Energy. Figure 46 shows the results of EDX for representative samples, panel a) shows the $2 \mathrm{H}-\mathrm{MoTe}_{2}$ and panel b) shows the results for $1 T^{\prime}-\mathrm{MoTe}_{2}$ sample. For both samples we notice that only peaks related to Molyb- 

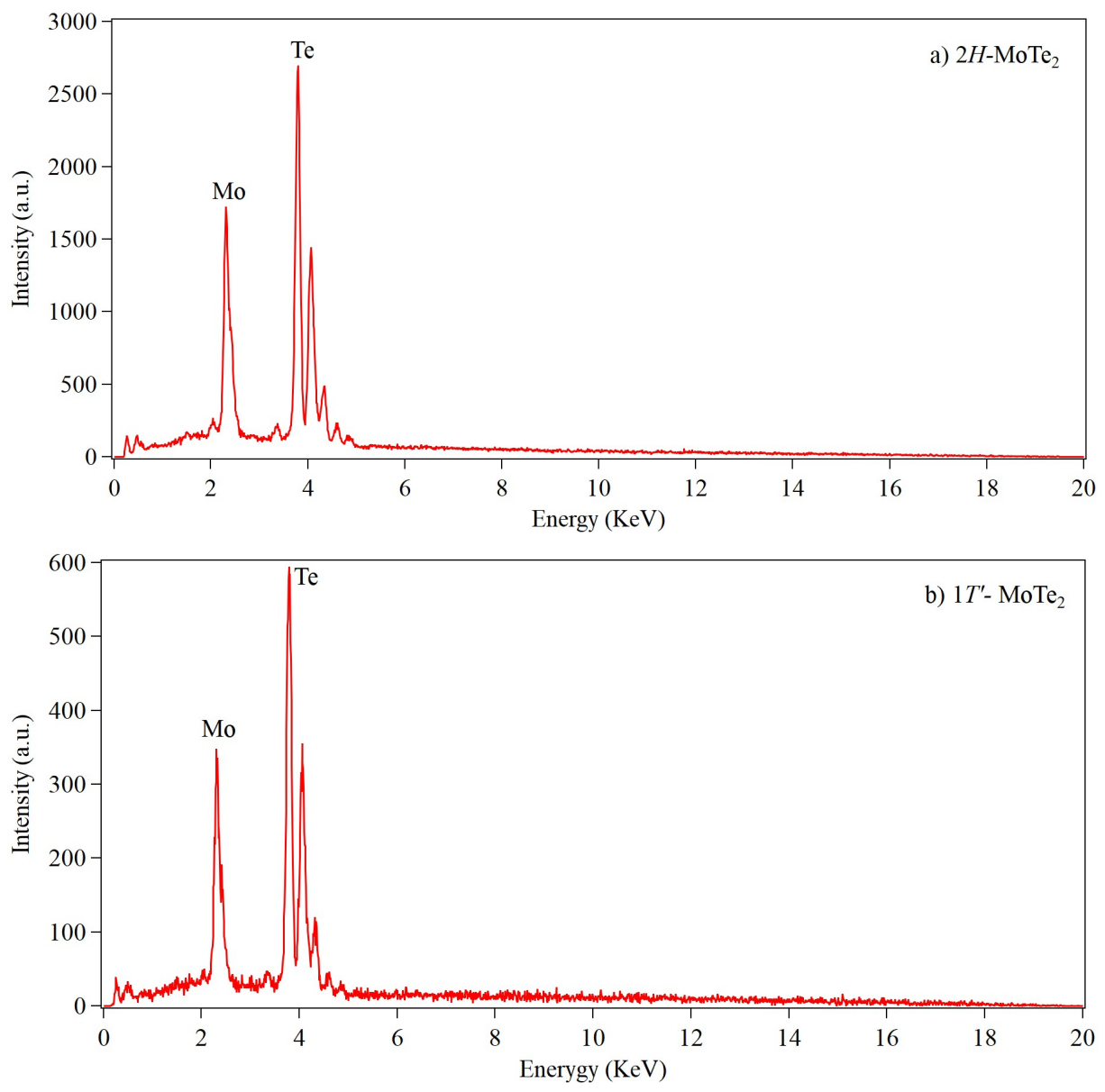

Figure 46. Stoichiometric analysis of a) $2 \mathrm{H}-\mathrm{MoTe}_{2}$ and b) $1 T^{\prime}-\mathrm{MoTe}_{2}$ by Energydispersive $\mathrm{x}$-ray spectroscopy (EDX).

denum (Mo) or Tellurium (Te) are present. This indicates that our samples are of good quality and do not contains contaminants. Figure 45 shows the Raman measurement results for powdered samples of $2 \mathrm{H}-\mathrm{MoTe}_{2}$ and $1 T^{\prime}-\mathrm{MoTe}_{2}$. The present study performed detailed crystal structure analysis $\mathrm{f} \mathrm{MoTe}_{2}$ materials by investigating the structural changes of the $2 H-\mathrm{MoTe}_{2}$ and $1 T^{\prime}-\mathrm{MoTe}_{2}$ phases with respect to temperature. EDX spectroscopy was used to study the elements present in the $\mathrm{MoTe}_{2}$ samples. EDX spectroscopy is commonly used to quantify the impurities in synthesized materials. It has the ability to characterize different elements due to the fact that the element unique atomic structure produces a set of peaks on its electromagnetic emission spectrum. 
In the current study, EDX spectroscopy was performed on two samples. The first sample represented the $2 \mathrm{H}-\mathrm{MoTe}_{2}$ phase, and the second sample represented the $1 T^{\prime}-\mathrm{MoTe}_{2}$ phase. The EDX results for both samples, presented in Figure 46 a and 46b), show different elements by their dispersive energy plotted as peaks on an EDX spectrum. Both Figures (46a and 46b) show peaks related to molybdenum (Mo) and tellurium $(\mathrm{Te})$. There was no other peaks present which indicates that the samples were clear from impurities. These results confirm that the elemental composition of both samples consisted only of molybdenum (Mo) and tellurium (Te). Impurities in the samples would have introduced new peaks in the x-ray powder diffraction, which would have affected the Rietveld analysis. The EDX spectroscopy was an important step to evaluate the purity of the studied samples.

\subsection{Experimental Methods}

\subsubsection{X-ray Diffraction}

In order to determine the structural model we used high-resolution x-ray powder diffraction. Data were acquired at two temperatures $295 \mathrm{~K}$ and $100 \mathrm{~K}$ using the mailin program at beamline 11-BM of the Advanced Photon Source (APS) at Argonne National Laboratory. Data scans were taken over a $2 \theta$ angular range from 0.5 to $50^{\circ}$ using a $\operatorname{Si}(111)$ double crystal monochromator at a wavelength $\lambda=0.4127 \AA$. For analyzing the powder diffraction data, we performed Rietveld refinement using the GSAS-II software suite [85]

\subsubsection{Neutron diffraction}

Temperature dependence neutron powder diffraction was performed at the Canadian Neutron Beam Centre (CNBC) at Chalk River Laboratories using C2 and C5

diffractometers. Data scans using C2 diffractometer were taken over a $2 \theta$ angular range from 2.9 to $117^{\circ}$ at a wavelength $\lambda=1.3 \AA$ for temperatures from $5 \mathrm{~K}$ to 320 
K. Another set of scan were performed with wavelength $\lambda=2.37 \AA$ at temperatures from $5 \mathrm{~K}$ to $325 \mathrm{~K}$. Data scans using C5 diffractometer were taken over a $2 \theta$ angular range from 4.5 to $120^{\circ}$ at a wavelength $\lambda=2.44 \AA$ over a range of temperatures from $1.77 \mathrm{~K}$ to $293 \mathrm{~K}$.

\subsection{Results}

The results of high resolution x-ray diffraction experiments, performed at 11-BM, APS and neutron powder diffraction, Chalk River, on both $2 H-\mathrm{MoTe}_{2}$ and $1 T^{\prime}$ $\mathrm{MoTe}_{2}$ are presented here.

\subsubsection{H-MoTe 2 Powder Diffraction}

\subsubsection{Synchrotron X-rays}

APS synchrotron x-ray diffraction data collected from $2 \mathrm{H}-\mathrm{MoTe}_{2}$ is presented in Figure 47. The diffractogram shows intensity versus the scattering angle 2 $\theta$. Figure 47 , a) shows the x-ray diffraction pattern for $2 \mathrm{H}-\mathrm{MoTe}_{2}$ phase at $100 \mathrm{~K}$ and 295 $\mathrm{K}$. The difference between the diffraction patterns is shown in panel b) of the same Figure. The major difference between the $295 \mathrm{~K}$ and $100 \mathrm{~K}$ data is the peak height mismatch at $2 \theta$ value from $2.5^{\circ}$ to $15^{\circ}$. The peak at $3.4^{\circ}$ shows the largest peak height difference between the two diffraction patterns. Figure 48, Tables 13 and 14 summarize the attempted Rietveld refinement analysis for $2 \mathrm{H}-\mathrm{MoTe}_{2}$ phase. The Rietveld refinement of the x-ray powder diffraction experiment data from 11-BM was performed using GSAS-II [85]. Figure $48 \mathrm{a}$ and $\mathrm{b}$ for temperatures $295 \mathrm{~K}$ and $100 \mathrm{~K}$ respectively. The calculated diffraction pattern using GSAS-II software is shown in green curve, the measured x-ray diffraction pattern is in red. The difference between the calculated and the measured patterns is in blue, the background is shown in black. The blue tick marks represent the Bragg angles based on the modeled lattice symmetry studied with the Rietveld analysis. 
Table 13. Proposed model for $2 \mathrm{H}-\mathrm{MoTe}_{2}$ based on 11-BM XRD at 295K. Space Group $P 6_{3} / m m c a=3.51981(3) \AA, c=13.96812(7)$ A unit cell volume $=149.867(2)$ $\AA^{3}$.

\begin{tabular}{lccccc}
\hline \multicolumn{5}{c}{$2 H-\mathrm{MoTe}_{2}$} \\
\hline Atom & $\mathrm{x}$ & $\mathrm{y}$ & $\mathrm{z}$ & Occup. & $\mathrm{U}_{\text {iso }}\left(\AA^{2}\right)$ \\
Mo & $1 / 3$ & $2 / 3$ & $1 / 4$ & $0.962(3)$ & $0.0059(2)$ \\
Te & $1 / 3$ & $2 / 3$ & $0.6205(1)$ & $0.958(3)$ & $0.0070(2)$ \\
Coordination & & & & \\
$d_{M o-M o}(\AA)$ & $3.51981(0)$ & & & \\
$d_{M o-T e}(\AA)$ & $2.7204(5)$ & $3.51981(0)$ & & \\
$d_{T e-T e}(\AA)$ & $80.620(17)$ & & \\
$a_{M o-T e-M o}($ deg. $)$ & & & & \\
$a_{T e-M o-T e}($ deg.) & $83.34(3)$ & & & \\
$\mathrm{wR}_{P}=0.1690$ & $\mathrm{R}_{P}=0.1021$ & $\mathrm{GoF}=3.00$ & $\chi^{2}=0.0899$ & \\
\hline
\end{tabular}

Table 14. Proposed model for $2 H-\mathrm{MoTe}_{2}$ from 11-BM XRD at $100 \mathrm{~K}$. Space Group $P 6_{3} / m m c a=3.51473(2) \AA, c=13.94093(7)$ A unit cell volume $=149.144(2) \AA^{3}$.

\begin{tabular}{lccccc}
\hline \multicolumn{5}{c}{$2 H-\mathrm{MoTe}_{2}$} \\
\hline Atom & $\mathrm{x}$ & $\mathrm{y}$ & $\mathrm{z}$ & Occup. & $\mathrm{U}_{\text {iso }}\left(\AA^{2}\right)$ \\
Mo & $1 / 3$ & $2 / 3$ & $1 / 4$ & $0.998(3)$ & $0.0007(2)$ \\
Te & $1 / 3$ & $2 / 3$ & $0.62022(4)$ & $0.993(3)$ & $0.0011(2)$ \\
Coordination & & & & \\
$d_{M o-M o}(\AA)$ & $3.51473(0)$ & & & \\
$d_{M o-T e}(\AA)$ & $2.7187(4)$ & & & \\
$d_{T e-T e}(\AA)$ & $3.51473(0)$ & & & \\
$a_{M o-T e-M o}($ deg. $)$ & $80.542(14)$ & & & \\
$a_{T e-M o-T e}($ deg. $)$ & $83.440(18)$ & & & \\
$\mathrm{wR}_{P}=0.1685$ & $\mathrm{R}_{P}=0.0837$ & $\mathrm{GoF}=2.94$ & $\chi^{2}=0.0866$ & \\
\hline
\end{tabular}

The lattice parameters $(a, b, c)$ and atomic positions $(\mathrm{x}, \mathrm{y}, \mathrm{z})$ that were refined during the analysis are presented in Tables 13 and 14 . The weighted profile R-factor $\left(R_{w}\right)$ and $\chi^{2}$ values indicate the quality of the Rietveld refinement analysis; at $295 \mathrm{~K}, R_{w}$ and $\chi^{2}$ were $0.1690,0.0899$, and at $100 \mathrm{~K}$ the values were 0.1685 , and 0.0866 . These values indicates the quality of the fit for the hexagonal symmetry with space group $P 6_{3} / m m c$. The lattice parameters, as well as the unit cell volume, increases with 
a)

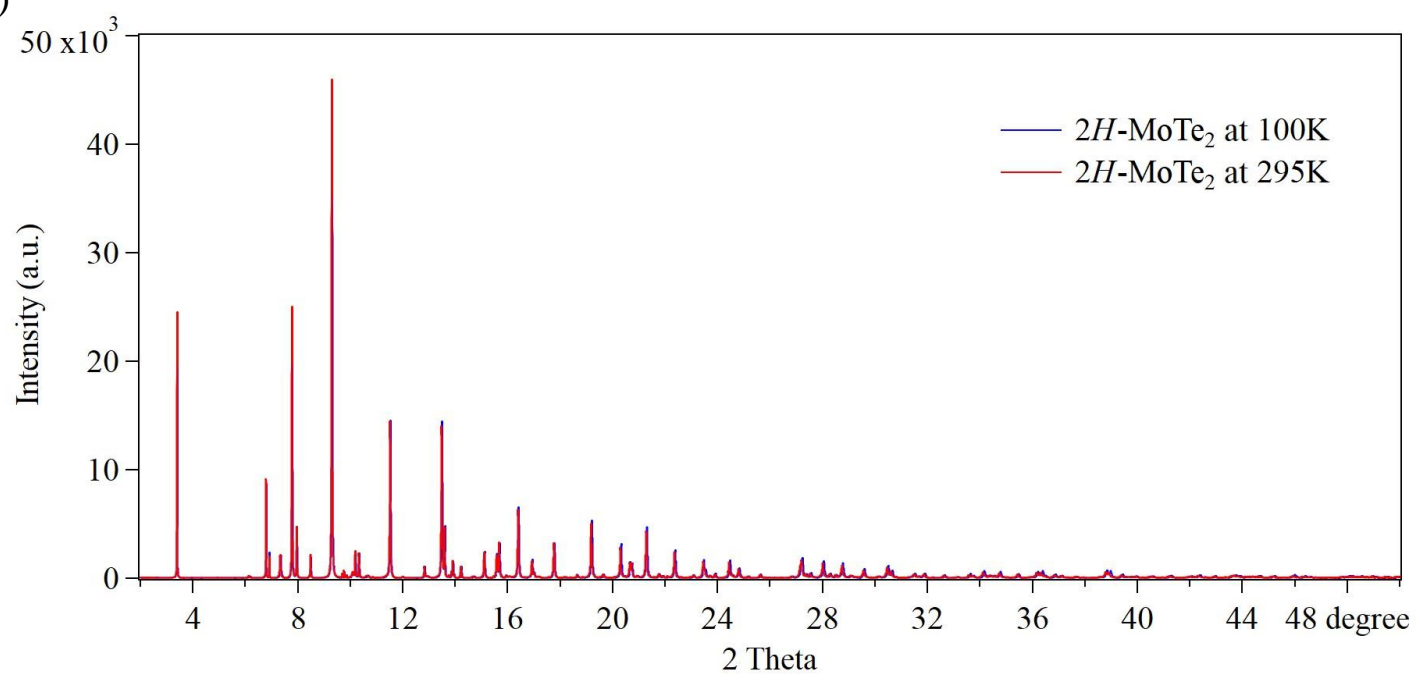

b)

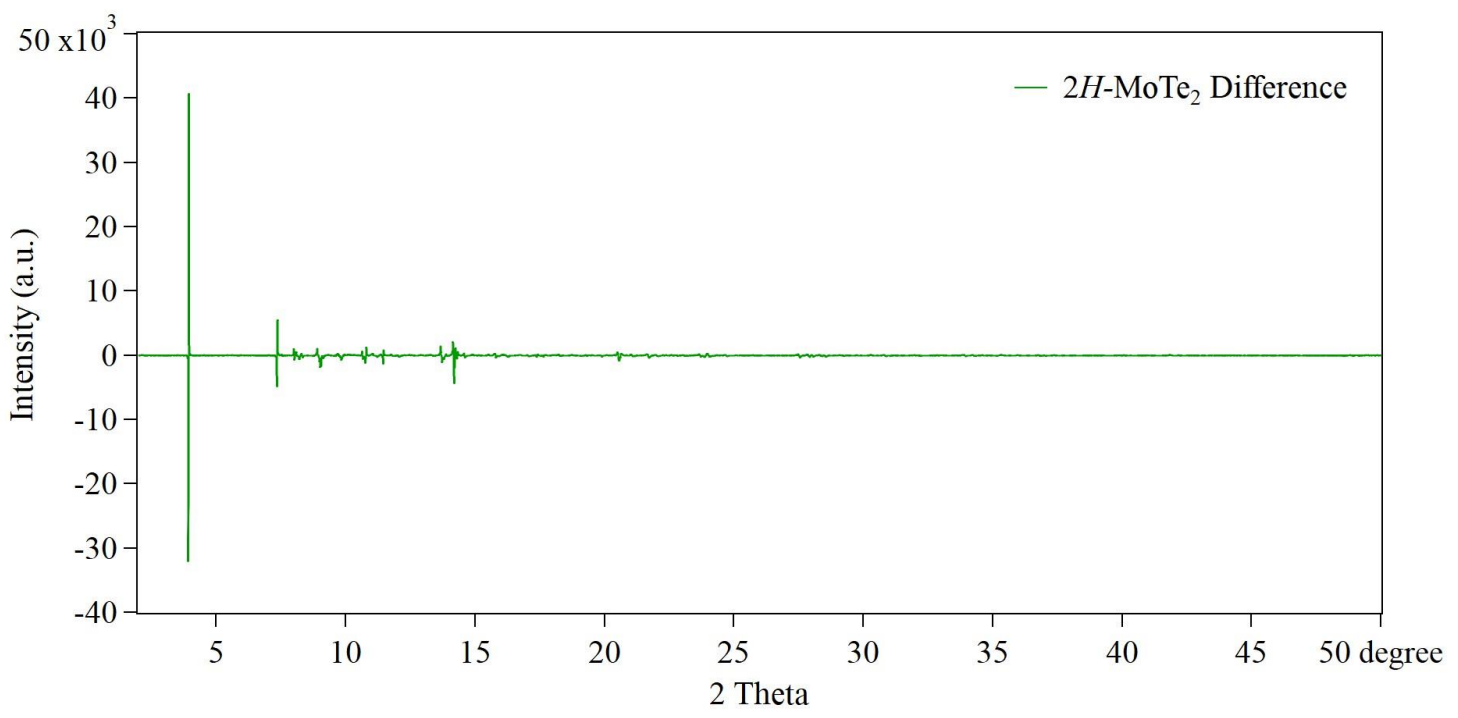

Figure 47. Panel a) presents the x-ray diffraction pattern from 11-BM at APS of $2 \mathrm{H}-\mathrm{MoTe}_{2}$ at $295 \mathrm{~K}$ and $100 \mathrm{~K}$. Panel b) shows the diffraction difference between the patterns.

temperature from $100 \mathrm{~K}$ to up to room temperature. The change in unit cell volume and the lattice parameters are due thermal expansion at higher temperature. The atomic positions $(\mathrm{x}, \mathrm{y}, \mathrm{z})$ and the atomic occupancy changed slightly temperature. The atomic occupancies, for molybdenum and tellurium, decreased when the temperature increased from $100 \mathrm{~K}$ to room temperature. A higher occupancy value will represent higher possibility for this particular atomic site to be occupied with a certain atom, 
a)

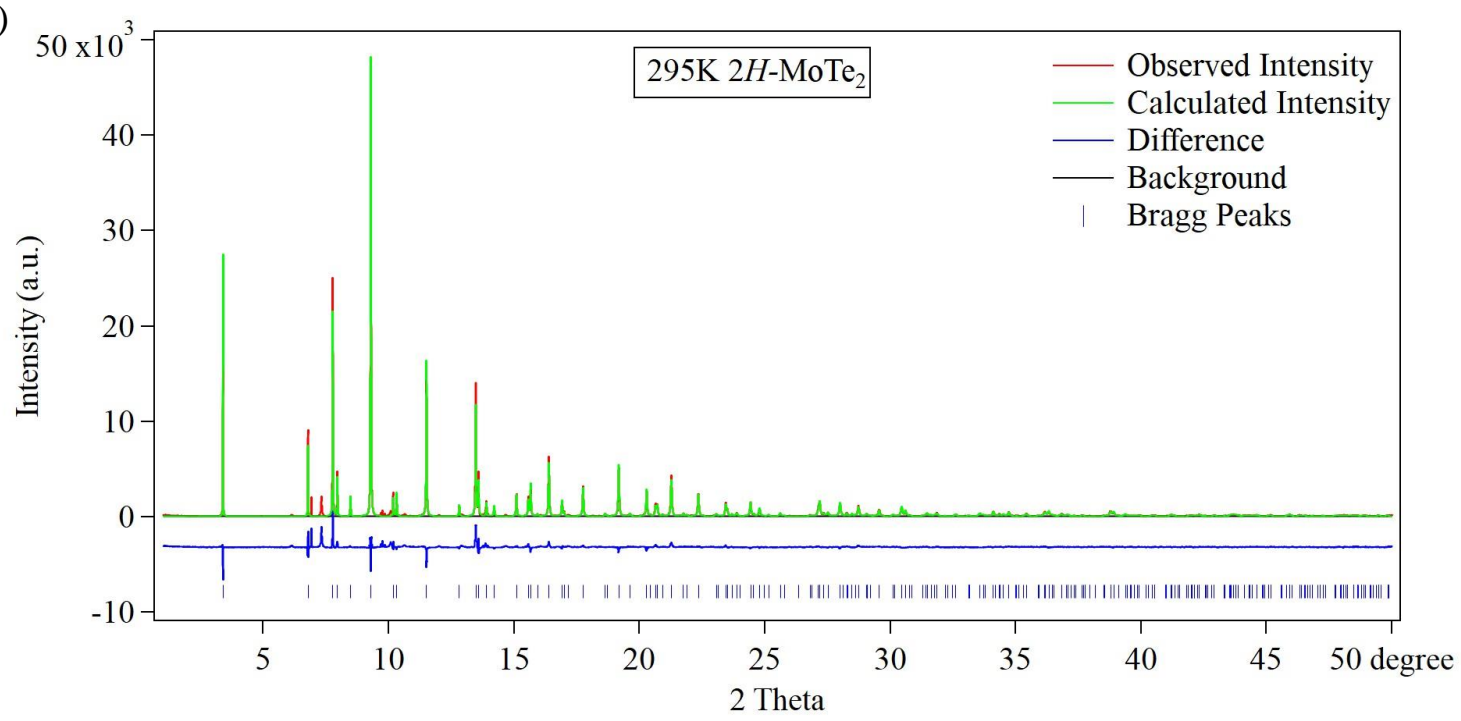

b)

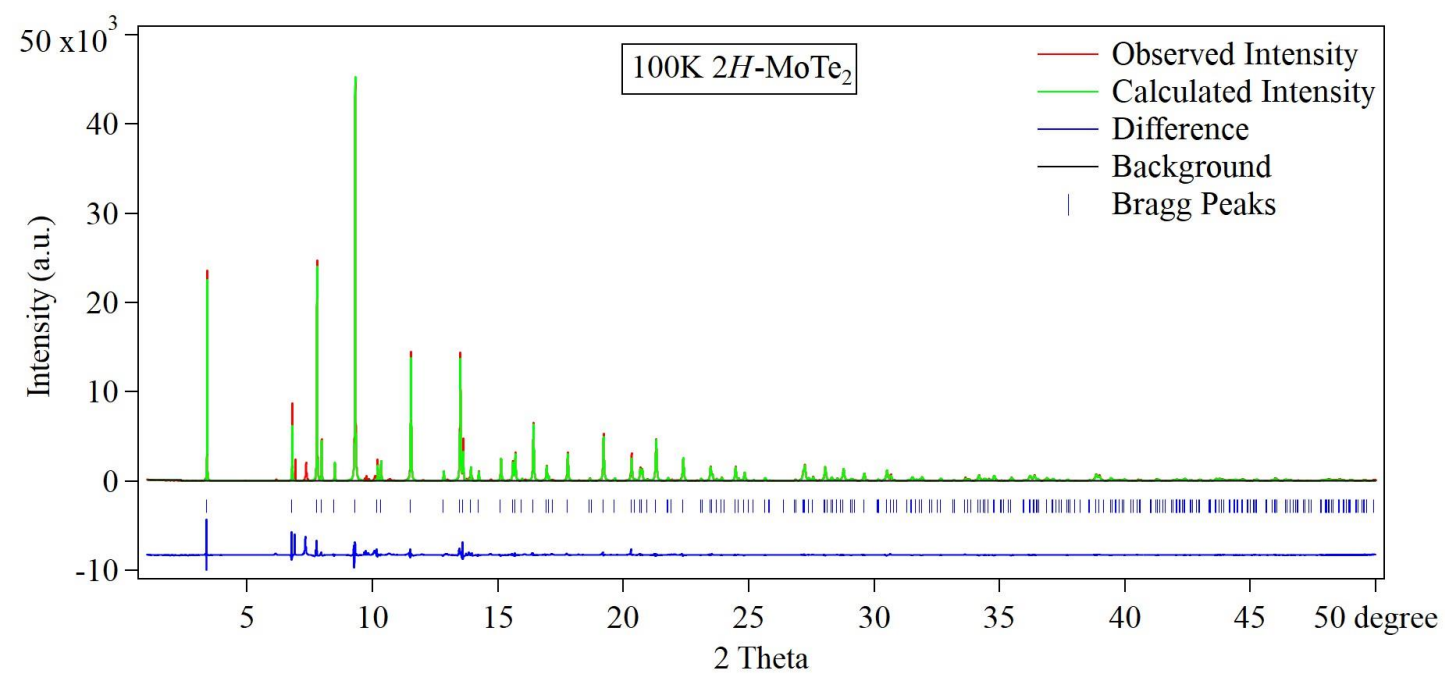

Figure 48. Rietveld refinement profiles of x-ray diffraction data from 11-BM at APS of $2 \mathrm{H}-\mathrm{MoTe}_{2}$. a) At $\left.300 \mathrm{~K}, \mathrm{~b}\right)$ at $100 \mathrm{~K}$.

and the lower possibility for vacancies in the atomic site.

\subsubsection{Neutron Powder Diffraction}

Temperature dependent neutron powder diffraction patterns for $2 \mathrm{H}-\mathrm{MoTe}_{2}$ collected at $\mathrm{C} 2$ diffractometer of $\mathrm{CNBC}$ is shown in Figure 49 where the diffraction pattern of $2 \mathrm{H}-\mathrm{MoTe}_{2}$ material are shown at temperatures 5, 200 and $320 \mathrm{~K}$ using a neutron wavelength $\lambda=1.3 \AA$. 
a)

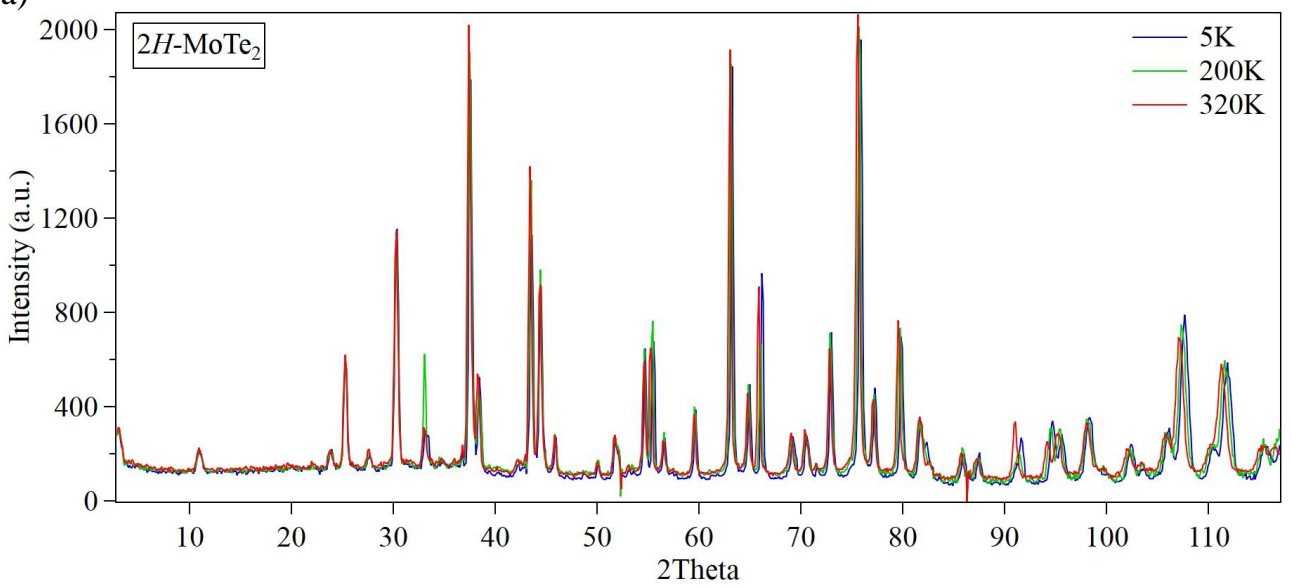

b)

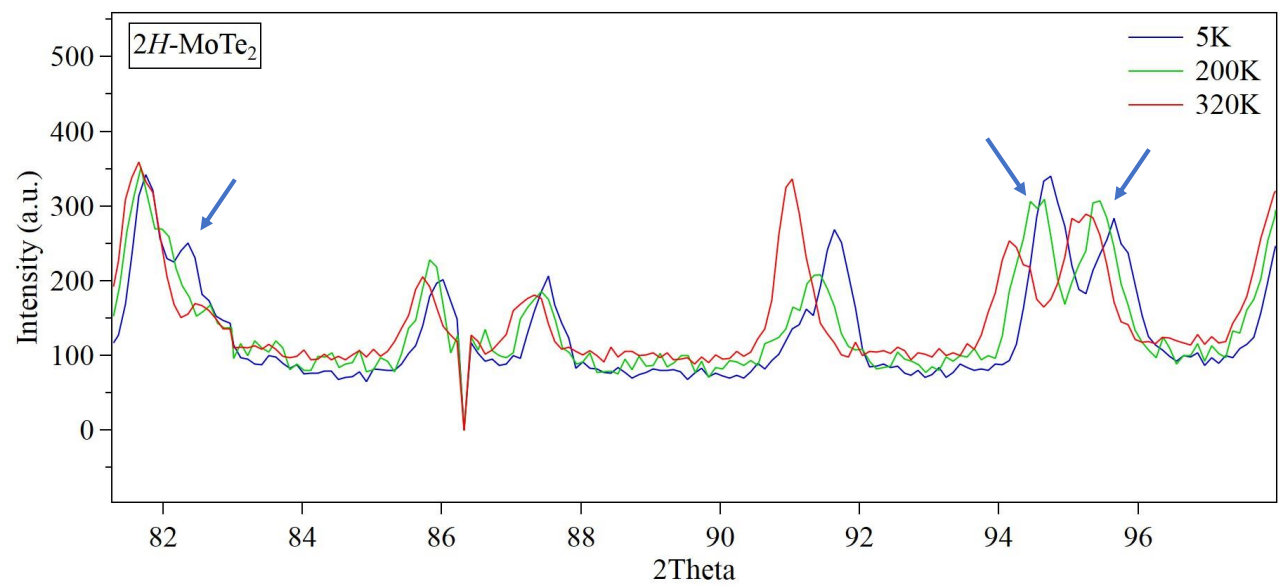

Figure 49. Panel a) shows $2 \mathrm{H}-\mathrm{MoTe}_{2}$ neutron powder diffraction data from $\mathrm{C} 2$ diffractometer at Chalk river with $\lambda=1.3 \AA$ performed at temperatures 5, 200 and $320 \mathrm{~K}$. Panel b) shows magnification of the diffraction pattern highlighting the $2 \theta$ from $81.5^{\circ}$ to $97.5^{\circ}$.

At $2 \theta=3^{\circ}$ the are no shift in the peaks. At $2 \theta=82.35^{\circ}$ for $\mathrm{T}=5 \mathrm{~K}$ we can observe a new peak which is not presented in the 200 and $320 \mathrm{~K}$ data. At $2 \theta=$ $94^{\circ}$ and $95^{\circ}$ there are 2 peaks (twin) at $320 \mathrm{~K}$ the first peak has higher intensity, at $200 \mathrm{~K}$ they are almost equal and at $5 \mathrm{~K}$ the peak at $2 \theta=95^{\circ}$ has higher intensity. Overall, most peaks sharpen and shift toward higher $2 \theta$ as the temperature increases as is expected for a thermal contraction of the lattice. Neutron powder diffraction patterns using wavelength $\lambda=2.37 \AA$ at temperatures 5, 16, 132, 200, 208, 291 and 
$320 \mathrm{~K}$ are shown in Figure 50. The diffractograms in this figure over different angular ranges: data collected at temperatures 16, 132, 208 and $291 \mathrm{~K}$ have $2 \theta$ range from $2.9^{\circ}$ to $83^{\circ}$ and data collected at temperatures 5,200 and $320 \mathrm{~K}$ have a $2 \theta$ range from $2.9^{\circ}$ to $117^{\circ}$

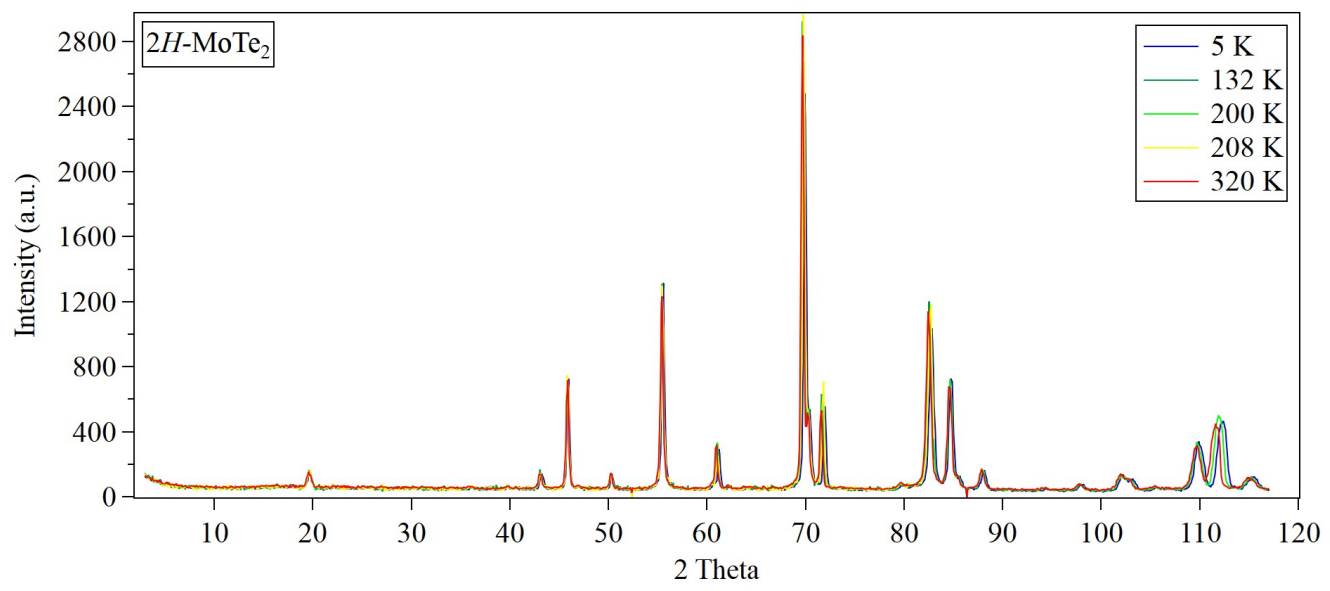

Figure 50. 2H-MoTe 2 neutron powder diffraction data from C2 diffractometer at Chalk river with $\lambda=2.37 \AA$ performed at 5, 132, 200, 208 and $320 \mathrm{~K}$.

\subsubsection{1 $1 T^{\prime}-\mathrm{MoTe}_{2}$ Powder Diffraction}

\subsubsection{Synchrotron X-rays}

High-resolution x-ray diffraction patterns of the $1 T^{\prime}-\mathrm{MoTe}_{2}$ phase collected at $100 \mathrm{~K}$ and $295 \mathrm{~K}$ is shown in Figure 51, in panel a) the full x-ray powder diffraction pattern for both temperatures are superimposed. The lower scattering angle range up to $2 \theta=15^{\circ}$ showed the largest differences between the two patterns. Panel b) shows the difference between the two patterns where peaks at angular $2 \theta=3.4,6.8$, and $13.7^{\circ}$ represents the highest peak intensity differences.

Data presented over specific scattering angular ranges in order to highlight the differences between the $1 T^{\prime}-\mathrm{MoTe}_{2}$ at low and room temperature as shown in Figure 52 , all panels. In this figure low temperature is shown blue line and room temperature data is shown by a red line. Panel a show the height difference and peak position shift 
a)

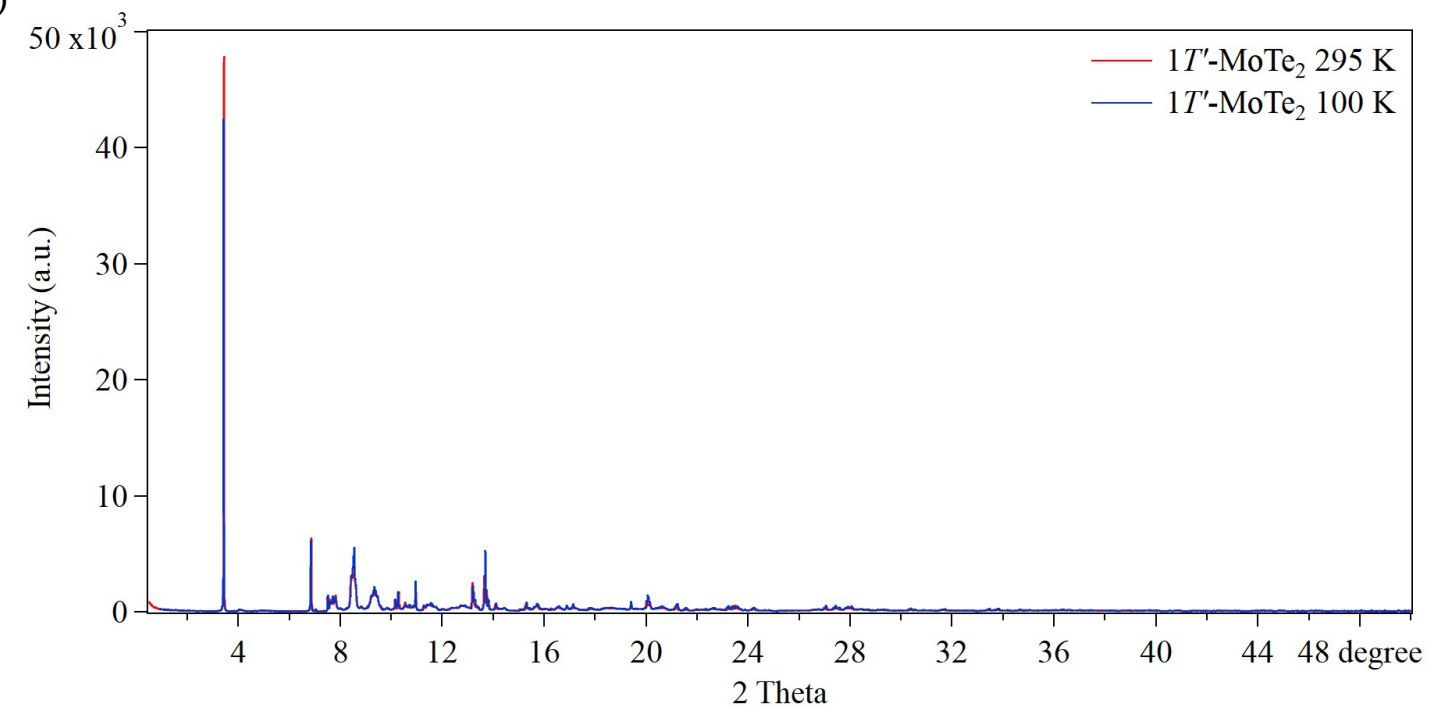

b)

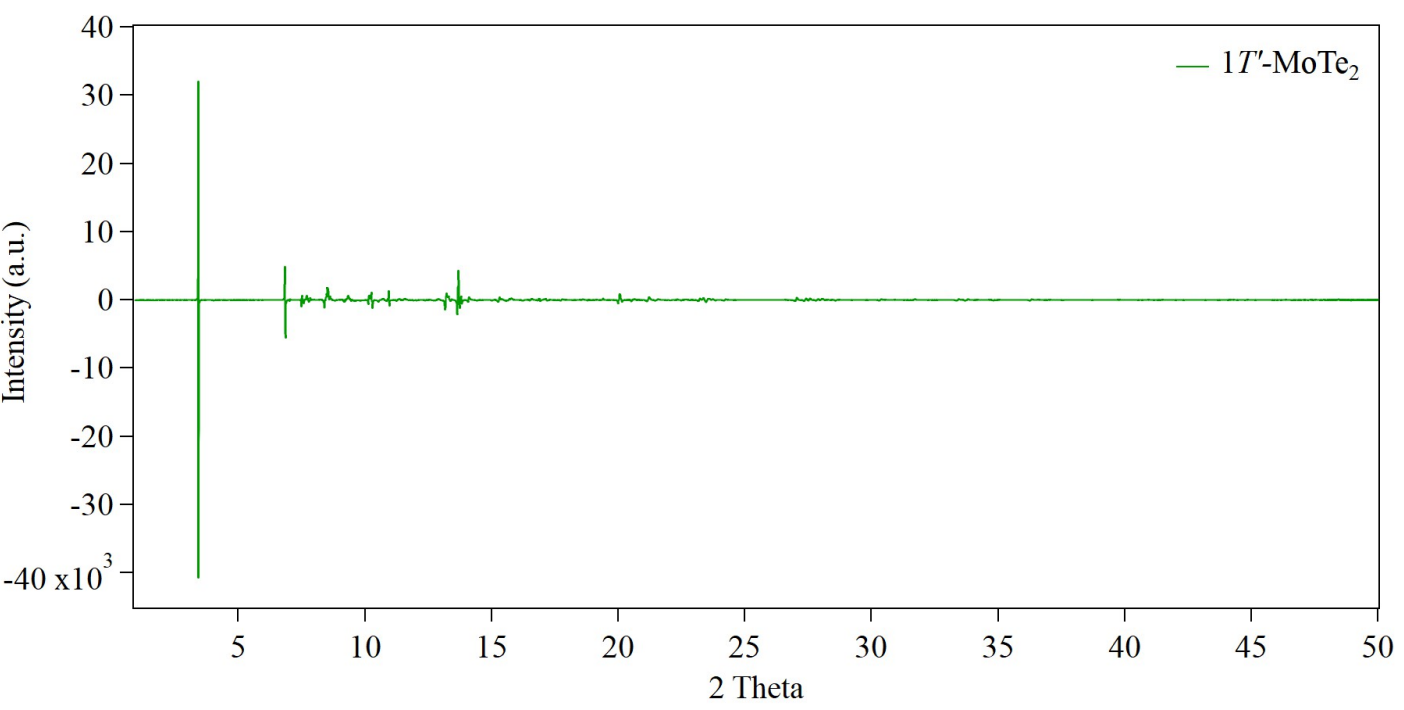

Figure 51. X-ray diffraction pattern from 11-BM at APS of $1 T^{\prime}-\mathrm{MoTe}_{2}$ at $295 \mathrm{~K}$ and $100 \mathrm{~K}$. Panel a) shows the full diffraction pattern and panel b) shows the difference between the patterns.

in the highest intensity peak in the diffractogram in the $1 T^{\prime}-\mathrm{MoTe}_{2}$ phase. Panel b focused on the angular range $2 \theta$ from $6.5^{\circ}$ to $17^{\circ}$, where we see new peaks emerged at $100 \mathrm{~K}$ at $2 \theta=7.7,10.7^{\circ}$. These new peaks are related to the structural phase transition from $1 T^{\prime}-\mathrm{MoTe}_{2}$ to $T_{d^{-}} \mathrm{MoTe}_{2}$. Panel c contains data collected over the angular range $2 \theta$ from $16^{\circ}$ to $26^{\circ}$; we can see that a new peak raised at low temperature at $2 \theta=23.36^{\circ}$. Other noticeable change is that some peaks merge into one peak for 

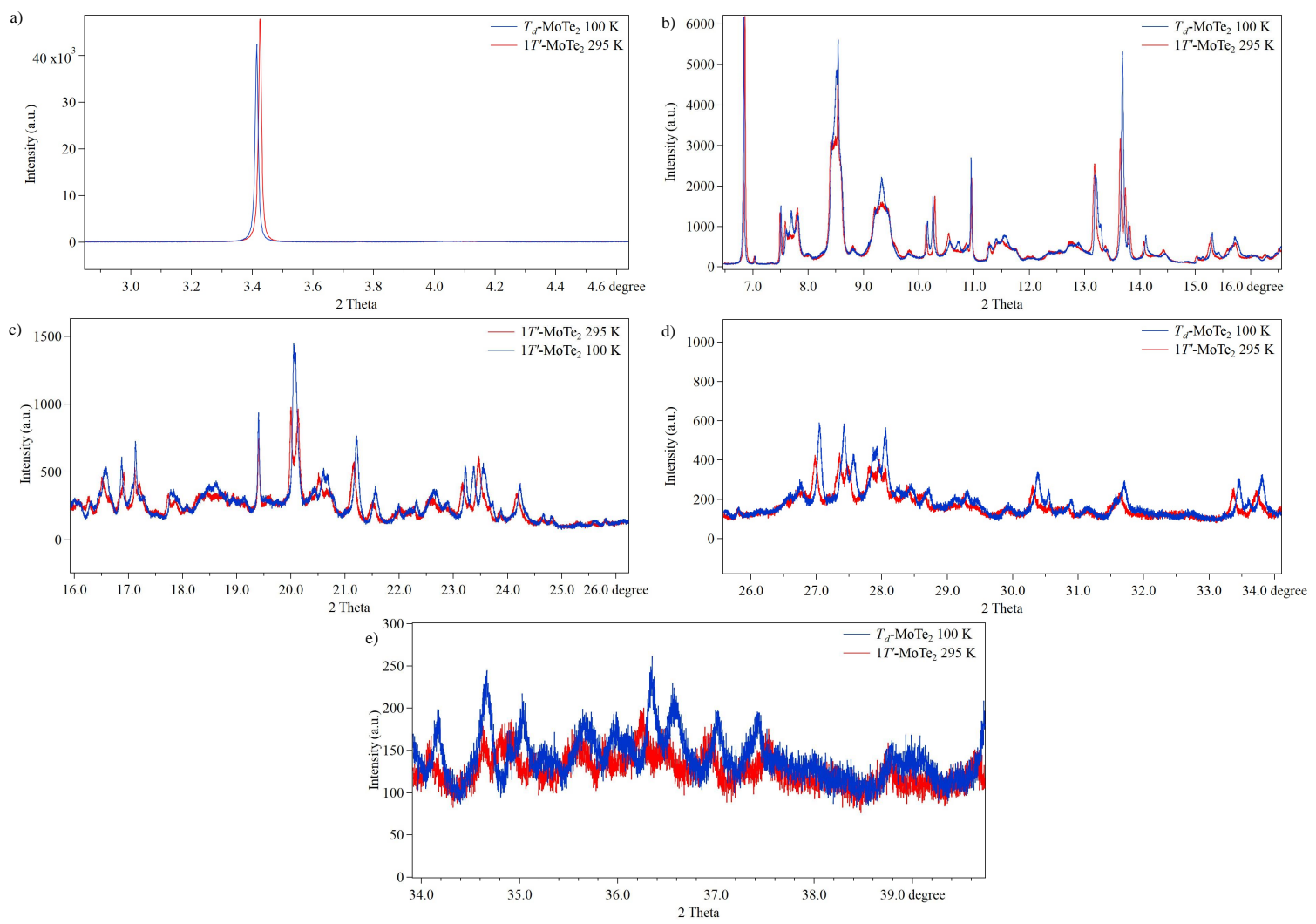

Figure 52. Focused section for the comparison from the x-ray diffraction pattern of data taken at $11-\mathrm{BM}$ at $\mathrm{APS}$, of $1 T^{\prime}-\mathrm{MoTe}_{2}$ at $295 \mathrm{~K}$ (red) and $100 \mathrm{~K}$ (blue). The full patterns are shown in figure 51 .

example the two peaks around $2 \theta=20^{\circ}$ at room temperature merge into one at low temperature. Panel $\mathrm{d}$ focus on the diffraction from $2 \theta=26$ to $34^{\circ}$ where two peaks at $2 \theta=27.79^{\circ}$ and $27.98^{\circ}$ merged to one peak located at $2 \theta=28.89^{\circ}$. In panel e new peaks emerge at low temperatures located at angular $2 \theta=36.4$ and $36.7^{\circ}$.

The $1 T^{\prime}-\mathrm{MoTe}_{2}$ proposed model lattice parameters and atomic positions are presented in Tables 15 and 16 . The $1 T^{\prime}-\mathrm{MoTe}_{2}$ phase is reported $[157,158]$ to undergo a structural phase transition at temperature below 200 K. For this reason we collected $\mathrm{x}$-ray powder diffraction data at $100 \mathrm{~K}$ which is well below the expected transition temperature.

The difference between $1 T^{\prime}-\mathrm{MoTe}_{2}$ and $T_{d}-\mathrm{MoTe}_{2}$ crystal symmetry is mainly the value of angle $\beta$ which changes changes from $90.0^{\circ}$ in orthorohmbic $T_{d}$ to $92.54889^{\circ}(19)$ in 
Table 15. Proposed model for $1 T^{\prime}-\mathrm{MoTe}_{2}$ from 11-BM XRD at 295K. Space Group $P 2_{1} / m a=6.32049(19) \AA, b=3.47182(14) \AA, c=13.83659(27) \AA, \beta=92.5489^{\circ}(19)$, unit cell volume $=303.325(13) \AA^{3}$.

\begin{tabular}{|c|c|c|c|c|c|}
\hline \multicolumn{6}{|c|}{$1 T^{\prime}-\mathrm{MoTe}_{2}$} \\
\hline Atom & $\mathrm{x}$ & $\mathrm{y}$ & $\mathrm{Z}$ & Occup. & $\mathrm{U}_{i s o}\left(\AA^{2}\right)$ \\
\hline Mo1 & $0.1748(8)$ & $1 / 4$ & $0.0051(5)$ & $0.432(4)$ & $0.0060(10)$ \\
\hline Mo2 & $0.3468(13)$ & $-1 / 4$ & $0.5087(6)$ & $0.329(4)$ & $0.0037(18)$ \\
\hline Te1 & $0.5907(8)$ & $1 / 4$ & $0.1091(3)$ & $0.530(4)$ & $0.0129(13)$ \\
\hline Te2 & $0.1026(9)$ & $-1 / 4$ & $0.1493(4)$ & $0.407(5)$ & $0.0054(17)$ \\
\hline Te3 & $0.5833(11)$ & $-1 / 4$ & $0.3584(5)$ & $0.401(5)$ & $0.0354(28)$ \\
\hline $\mathrm{Te} 4$ & $0.0423(7)$ & $2 / 3$ & $0.6202(4)$ & $0.562(5)$ & $0.0223(16)$ \\
\hline Coordination & & & & & \\
\hline Atomic distances & & & & & \\
\hline$d_{M o 1-M o 1}(\AA)$ & $2.818(8)$ & & & & \\
\hline$d_{M o 2-M o 2}(\AA)$ & $2.605(13)$ & & & & \\
\hline$d_{T e 1-T e 1}(\AA)$ & $3.611(7)$ & & & & \\
\hline$d_{T e 2-T e 2}(\AA)$ & $4.601(11)$ & & & & \\
\hline$d_{T e 3-T e 3}(\AA)$ & $4.458(13)$ & & & & \\
\hline$d_{T e 4-T e 4}(\AA)$ & $3.309(7)$ & & & & \\
\hline$d_{M o 1-T e 1}(\AA)$ & $2.806(7)$ & & & & \\
\hline$d_{M o 1-T e 2}(\AA)$ & $2.704(7)$ & & & & \\
\hline$d_{M o 2-T e 3}(\AA)$ & $2.557(8)$ & & & & \\
\hline$d_{M o 2-T e 4}(\AA)$ & $2.942(7)$ & & & & \\
\hline Bond angles & & & & & \\
\hline$a_{T e 1-M o 1-T e 1}$ (deg.) & $117.02(19)$ & & & & \\
\hline$a_{T e 2-M o 1-T e 2}(\mathrm{deg})$. & $76.76(16)$ & & & & \\
\hline$a_{T e 3-M o 2-T e 3}(\mathrm{deg})$. & $119.4(3)$ & & & & \\
\hline$a_{T e 4-M o 2-T e 4}(\mathrm{deg})$. & $78.3(3)$ & & & & \\
\hline $\mathrm{wR}_{P}=0.3142$ & $\mathrm{R}_{P}=0.2334$ & $\mathrm{GoF}=5.07$ & $\chi^{2}=0.2565$ & & \\
\hline
\end{tabular}

the monoclinic $1 T^{\prime}$ (Figure $43 \mathrm{~d}$ and $\mathrm{f}$ ). The graphical presentation of the Rietveld refinement analysis of the $1 T^{\prime}-\mathrm{MoTe}_{2}$ phase x-ray powder diffraction is given in Figure 53. Panel a) shows the refinement at room temperature $295 \mathrm{~K}$ using the monoclinic symmetry and panel b) shows the $100 \mathrm{~K}$ data refined using the orthorhombic symmetry. 
Table 16. Proposed model for $T_{d}-\mathrm{MoTe}_{2}$ from 11-BM XRD at $100 \mathrm{~K}$. Space Group $P m n 2_{1} a=3.46675(29) \AA, b=6.30310(53) \AA, c=13.85899(39) \AA, \beta=90.0^{\circ}(0)$, unit cell volume $=302.837(31) \AA^{3}$. The fit quality here is low and the negative values of the $U_{\text {iso }}$ and the large fit quality parameters listed below.

\begin{tabular}{lccccc}
\hline & \multicolumn{7}{c}{$1 T^{\prime}-\mathrm{MoTe}_{2}$} & & \\
\hline Atom & $\mathrm{x}$ & $\mathrm{y}$ & $\mathrm{z}$ & Occup. & $\mathrm{U}_{\text {iso }}\left(\AA^{2}\right)$ \\
Mo1 & 0.0 & $0.6259(8)$ & $0.5075(4)$ & 1.0 & $-0.0029(14)$ \\
Mo2 & 0.0 & $0.0175(8)$ & $0.0132(4)$ & 1.0 & $-0.0053(11)$ \\
Te1 & 0.0 & $0.8579(7)$ & $0.6532(2)$ & 1.0 & $-0.0200(4)$ \\
Te2 & 0.0 & $0.6564(8)$ & $0.0994(3)$ & 1.0 & $0.0084(18)$ \\
Te3 & 0.0 & $0.3110(8)$ & $0.3584(5)$ & 1.0 & $0.0119(18)$ \\
Te4 & 0.0 & $0.2125(6)$ & $0.6202(4)$ & 1.0 & $-0.0151(4)$
\end{tabular}

Coordination

Atomic distances

$\begin{array}{lc}d_{M o 1-M o 1}(\AA) & 3.46675(0) \\ d_{M o 2-M o 2}(\AA) & 3.46675(0) \\ d_{T e 1-T e 1}(\AA) & 3.46675(0) \\ d_{T e 2-T e 2}(\AA) & 3.46675(0) \\ d_{T e 3-T e 3}(\AA) & 3.46675(0) \\ d_{T e 4-T e 4}(\AA) & 3.46675(0) \\ d_{M o 1-T e 1}(\AA) & 2.493(7) \\ d_{M o 1-T e 2}(\AA) & 2.792(6) \\ d_{M o 1-T e 3}(\AA) & 2.689(6) \\ d_{M o 1-T e 4}(\AA) & 2.992(7) \\ d_{M o 2-T e 1}(\AA) & 2.718(6) \\ d_{M o 2-T e 2}(\AA) & 2.571(8) \\ d_{M o 2-T e 3}(\AA) & 2.795(8) \\ d_{M o 2-T e 4}(\AA) & 2.740(5) \\ B 01) & \end{array}$

Bond angles

$a_{T e 1-M o 1-T e 2}($ deg.) $\quad 90.2(2)$

$a_{T e 1-M o 1-T e 3}$ (deg.) $\quad 121.38(19)$

$a_{T e 1-M o 2-T e 2}($ deg.) $\quad 85.64(19)$

$a_{T e 2-M o 2-T e 4}($ deg.) $\quad 78.12(17)$

$\mathrm{wR}_{P}=0.3502 \quad \mathrm{R}_{P}=0.2838 \quad \mathrm{GoF}=5.82 \quad \chi^{2}=0.3382$

\subsubsection{Neutron Powder Diffraction}

Figure 54 shows temperature dependence neutron powder diffraction pattern for the $1 T^{\prime}-\mathrm{MoTe}_{2}$ phase from the $\mathrm{C} 2$ diffractometer at the Canadian Neutron Beam 
a)

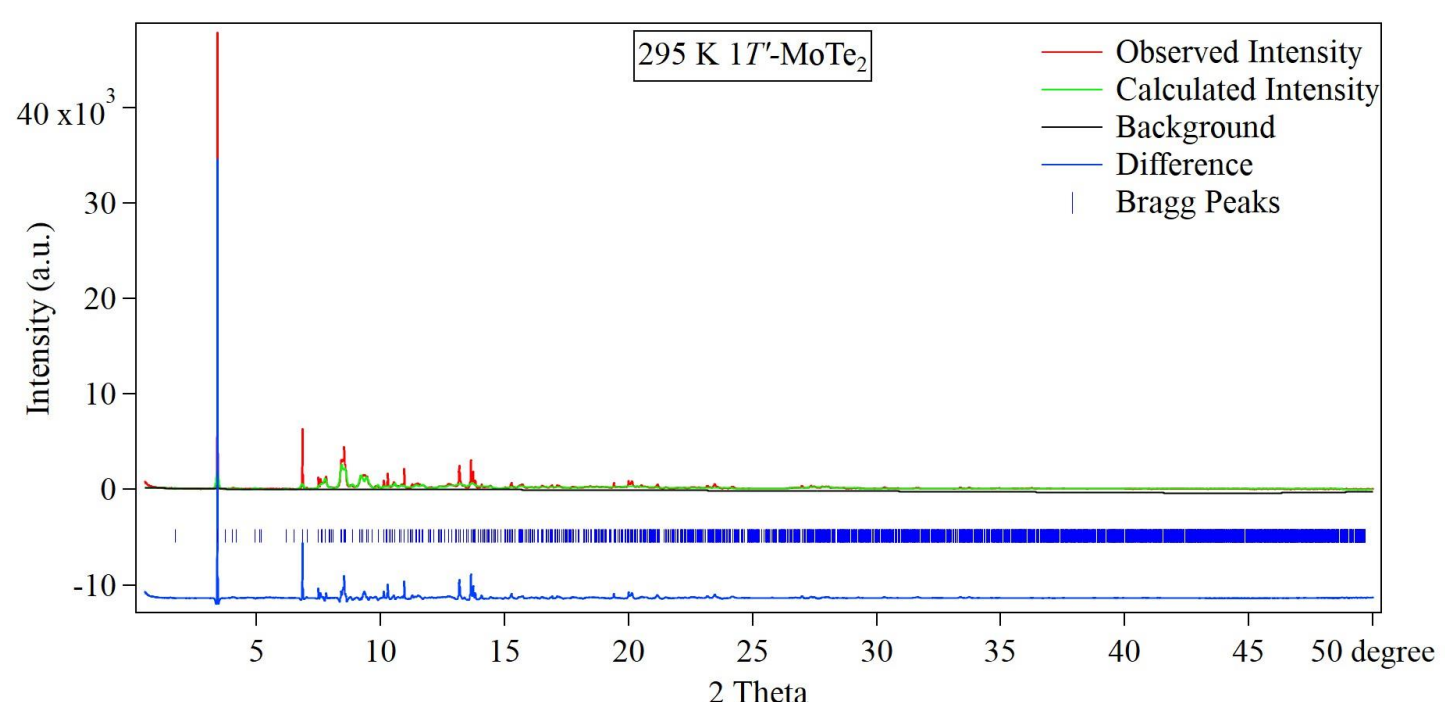

b)

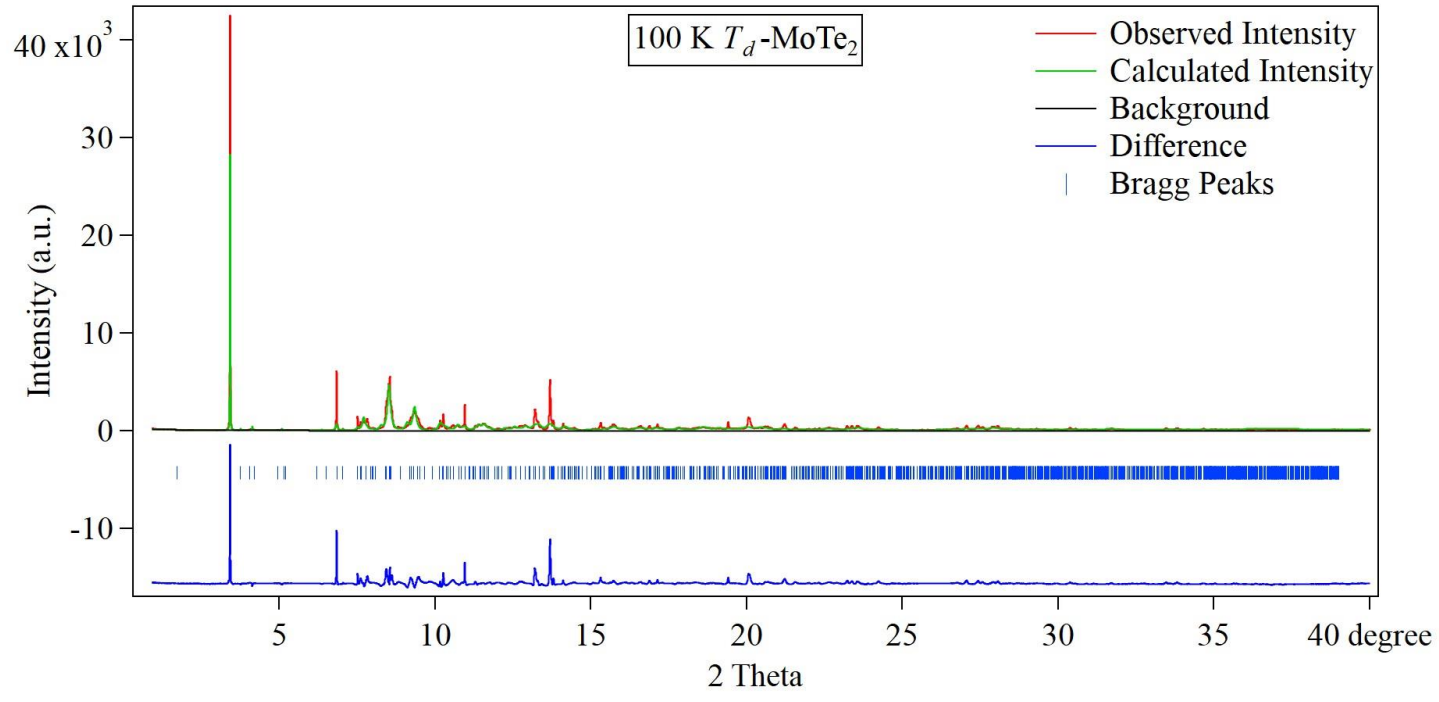

Figure 53. X-ray diffraction pattern from 11-BM at APS of $1 T^{\prime}-\mathrm{MoTe}_{2}$ At $295 \mathrm{~K}$ and $100 \mathrm{~K}$. Panel a) shows the full diffraction pattern where panels b-f shows focused portions of this pattern.

Centre. Samples were measured at the following temperatures 5, 20, 92, 113, 161, 200, 293, 320 and $325 \mathrm{~K}$ using a wavelength $\lambda=1.33 \AA$. The resulting diffraction patterns are shown in the upper panel of Figure 54. Temperatures 5, 200 and $320 \mathrm{~K}$ cover the full detector range in which the scattering angle $2 \theta$ varies from $3^{\circ}$ to $117^{\circ}$. While data for the temperatures 20, 113, 161 and $293 \mathrm{~K}$ cover a $2 \theta$ range from $3^{\circ}$ to $83^{\circ}$ and data for temperatures 20 and $325 \mathrm{~K}$ cover a $2 \theta$ range from $37^{\circ}$ to $117^{\circ}$. 
Peaks with $2 \theta$ greater than $30.469^{\circ}$ shift to higher $2 \theta$ values at low temperature.
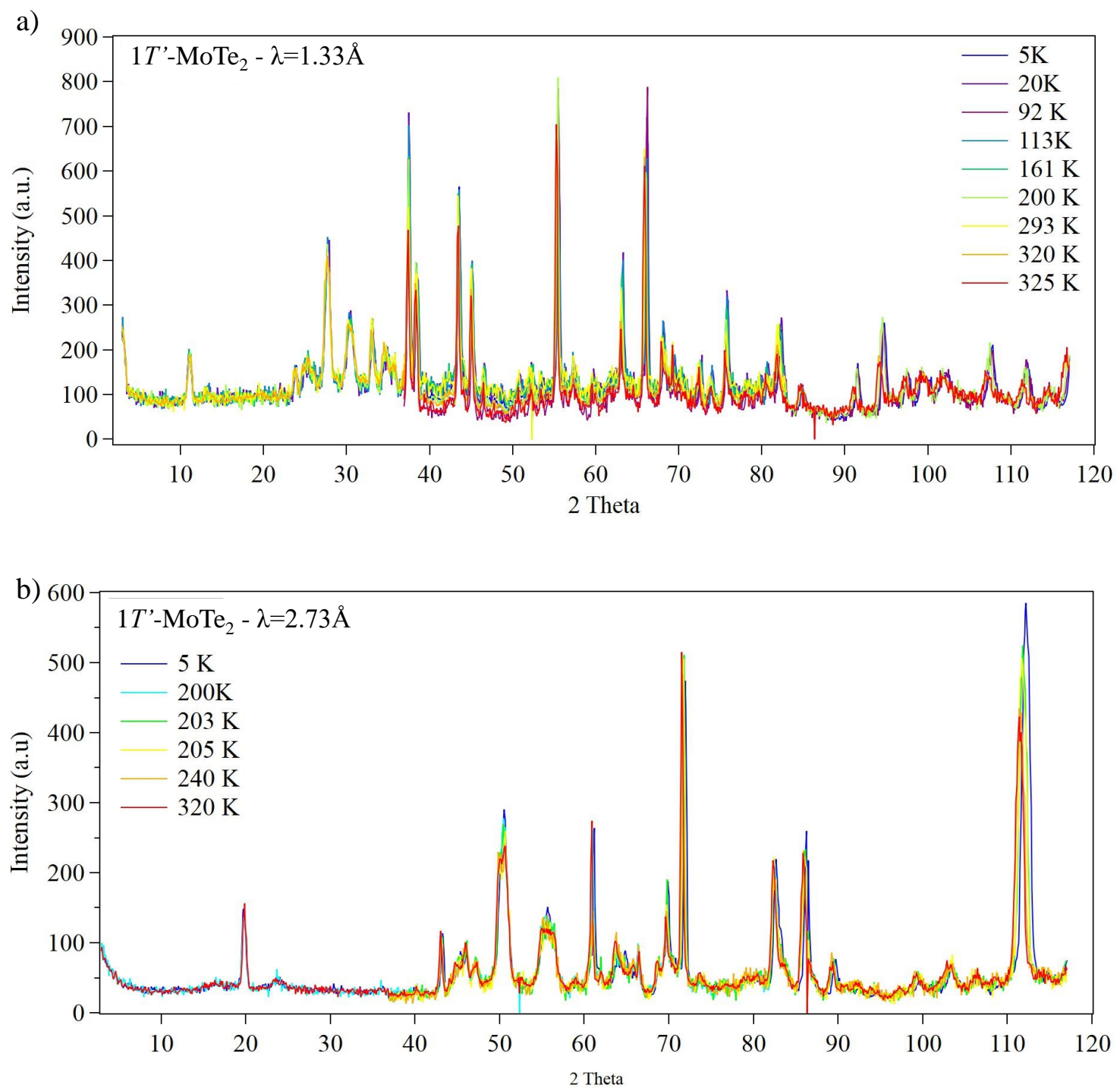

Figure 54. Panel a) $1 T^{\prime}-\mathrm{MoTe}_{2}$ temperature dependence neutron powder diffraction data from $\mathrm{C} 2$ diffractometer at Chalk River with $\lambda=1.33 \AA$. Panel b) $1 T^{\prime}-\mathrm{MoTe}_{2}$ temperature dependence neutron powder diffraction data from $\mathrm{C} 2$ diffractometer at Chalk River with $\lambda=2.37 \AA$.

The lower panel of Figure 54 shows measurements of $1 T^{\prime}-\mathrm{MoTe}_{2}$ phase at temperatures 5, 200, 203, 205, 240 and $320 \mathrm{~K}$ using a wavelength $\lambda=2.37 \AA$. Neutron powder diffraction patterns were taken at temperatures $5,320 \mathrm{~K}$ over the full detector range in which the scattering angle $2 \theta$ varied from $3^{\circ}$ to $117^{\circ}$. Diffraction patterns 


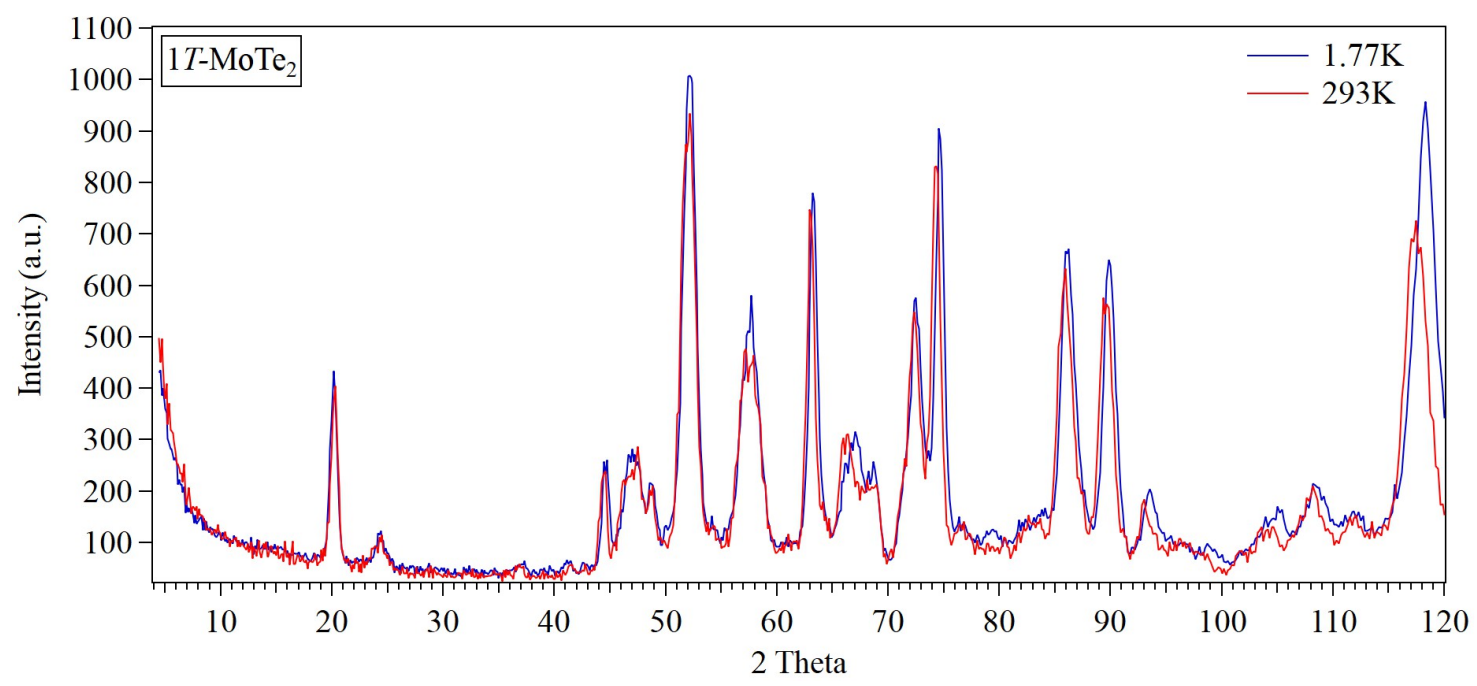

Figure 55. $1 T^{\prime}-\mathrm{MoTe}_{2}$ temperature dependence neutron powder diffraction data from C5 diffractometer at Chalk river with $\lambda=2.44 \AA$.

taken at temperatures 203,205 , and $240 \mathrm{~K}$ covered a $2 \theta$ range from $37^{\circ}$ to $117^{\circ}$. The measurement at $200 \mathrm{~K}$ covered angular range $2 \theta$ from $3^{\circ}$ to $83^{\circ}$. We observed two dips in the measurements one is at $2 \theta=52.33^{\circ}$ (except for temperature 203, $205 \mathrm{~K}$ no dip was found) and at $2 \theta=86.323^{\circ}$ for all temperatures. Peaks with $2 \theta$ greater than $60^{\circ}$ tended to shift to higher $2 \theta$ at lower temperatures. New peak appears at $5 \mathrm{~K}$ temperature at $2 \theta=64.862^{\circ}$, which is related to the structural phase transition from $1 T^{\prime}-\mathrm{MoTe}_{2}$ phase to $T_{d}-\mathrm{MoTe}_{2}$ phase.

Figure 55 shows temperature dependence neutron powder diffraction pattern for the $1 T^{\prime}-\mathrm{MoTe}_{2}$ phase from $\mathrm{C} 5$ diffractometer at Chalk river. We measured this sample at temperatures 1.77 and $293 \mathrm{~K}$ with wavelength $\lambda=2.44 \AA$. In this measurement, peaks from both temperatures coincides around or before $2 \theta=52.145^{\circ}$ which is the highest intensity peak. Peaks with $2 \theta$ greater than 62.954 shift to higher $2 \theta$ at low temperature. 


\subsection{Discussion}

\subsubsection{H-MoTe 2 Phase}

The primary structural analysis of $2 \mathrm{H}-\mathrm{MoTe}_{2}$ was performed using x-ray and neutron powder diffraction. These experiments provide structural characterization of atomic coordinates and detailed structural parameters of the particular material phase. The x-ray powder diffraction data obtained from the 11-BM measurement were analyzed using Rietveld analysis by GSAS-II suite. Rietveld analysis was used to identify the crystal structure and its symmetry as well as the lattice parameters, atomic positions, and atomic site occupancy at different temperatures. At room temperature, the crystal structure parameters were similar to values reported in literature $[123,159,160]$. Puotinen et al. performed x-ray powder diffraction and reported hexagonal system with space group $P 6_{3} / m m c$ with similar lattice parameters $a$ and $c$ [160]. They reported that their lowest $R_{W}$ value was $17 \%$, which is similar to our fit quality values, and attributed that to preferred orientation effects.

The diffraction indicates that $2 \mathrm{H}$-MoTe 2 sample has hexagonal crystal symmetry with space group $P 6_{3} / m m c$ remains the same at room temperature and $100 \mathrm{~K}$. We notice this fact by direct comparison of diffraction data collected at these temperatures (see Figure 47). The persistence of the diffraction pattern profile from room temperature to $100 \mathrm{~K}$, confirms that the bulk $2 \mathrm{H}-\mathrm{MoTe}_{2}$ maintains the same symmetry. Only a slight reduction in lattice parameters is observed upon lowering the temperature and this is expected to result from the lattice contraction.

Atomic positions values, for all atoms, increased slightly with temperature (Tables 13, and 14) while the occupancies of the atomic sites changed. At lower temperatures both atoms, Mo and Te, have less occupancy than room temperature.

We have performed a temperature dependence powder neutron diffraction experiment at Canadian Neutron Beam Centre, Chalk River. 2H-MoTe 2 was measured 
with different wavelengths for many temperatures (Figures 49 and 50). Except for a temperature based peak shift no major changes in the peaks, which was expected due to lack of phase transition in $2 \mathrm{H}-\mathrm{MoTe}_{2}$ phase.

\subsubsection{T' ${ }^{\prime}-\mathrm{MoTe}_{2}$ Phase}

Similar to $2 \mathrm{H}-\mathrm{MoTe}_{2}$, x-ray diffraction was performed on $1 \mathrm{~T}^{\prime}-\mathrm{MoTe}_{2}$ at room temperature and $100 \mathrm{~K}$. These temperatures were appropriate for the current study, since $100 \mathrm{~K}$ is lower than the reported phase transition temperature in $1 T^{\prime}-\mathrm{MoTe}_{2}$ phase. Rietveld refinement was used to analyze the diffraction experiment data and determine the structure of the material. Tables 15 and 16 showed the proposed crystal structure for the collected data before and after the structural phase transition. The analysis for $1 T^{\prime}-\mathrm{MoTe}_{2}$ at room temperature is confirmed to have a monoclinic symmetry. At $100 \mathrm{~K}$ the crystal symmetry was modeled as orthorhombic. Our low-temperature measurement at $100 \mathrm{~K}$ was below the phase transition temperature and the coexistence region [157], we expected that the $1 T^{\prime}-\mathrm{MoTe}_{2}$ would undergo a structural phase transition to $T_{d}-\mathrm{MoTe}_{2}$. This was further confirmed by our Rietveld refinement analysis. In $1 T^{\prime}-\mathrm{MoTe}_{2}$ the change from monoclinic to orthorhombic structure is based on a slight change in the lattice parameters. However, the symmetry change is thought to be mainly due to tilting in the $c$-axis by few degrees in the out-ofplane direction. In our measurements the tilting angle $\beta$ is about $2^{\circ}(\beta$ changes from $92.5489(19)^{\circ}$ to $\left.90.0^{\circ}\right)$ when the phase transitions from monoclinic to orthorhombic. It has been suggested that $\mathrm{MoTe}_{2}$ layers might easily shift due to the weak Van der

Waals forces [161]. Our findings further confirm the role the Van der Waals forces on the $\mathrm{MoTe}_{2}$ layers. The similarity of the layers and the ease of movement between them is ideal for symmetry change through the structural phase transition, this is also the reason of the coexistence region where some of both phases exits. Kim et al. showed that the slight change in the interlayer bonding did affect the bulk symmetry 
in $\mathrm{MoTe}_{2}[123]$.

We have considered several sample-related features that might hinder better Rietveld refinement quality. We performed measurements to check the quality of our samples and quantify the impurity level prior to diffraction experiments by using EDX and Raman spectroscopy. Our materials were powdered prior to all the measurements. We were concerned about the effect of powdering the single crystal on the quality of the crystal. It could have been possible that the weakly connected layers would shift or atoms might create vacancies within the crystal. However, studies on small single-crystals of $1 T^{\prime}-\mathrm{MoTe}_{2}$ indicates that layer shifting is already present [161]. The formation of oxides in all phases of $\mathrm{MoTe}_{2}$ was ruled out because the material was not expected to react with ambient atmosphere. Our results indicate that the weakly attached atoms may affect the crystallinity which can lead to reduced quality of the diffraction pattern quality make the pattern harder to refine. We compared our x-ray powder diffraction data to simulated patterns created by GSAS-II software. The data for phases $1 T^{\prime}-\mathrm{MoTe}_{2}$ and $T_{d}-\mathrm{MoTe}_{2}$ showed a large number of wide closely peaked peaks that resulted in very broad peaks instead of many well-defined peaks. The behavior of our bulk powdered $1 T^{\prime}-\mathrm{MoTe}_{2}$ and $T_{d}-\mathrm{MoTe}_{2}$ materials is similar to the powdered few-layer nano-sheets $1 T^{\prime}-\mathrm{MoTe}_{2}$ reported by Sun et al. (Figure 56). This behavior also impacted the attempt to refine data from $\mathrm{MoTe}_{2}$ phases, specially $1 T^{\prime}-\mathrm{MoTe}_{2}$ and $T_{d}-\mathrm{MoTe}_{2}$, although it is less in $2 \mathrm{H}-\mathrm{MoTe}_{2}$ phase.

Figures 54 and 55 showed the The temperature dependence powder neutron diffraction experiment at CNBC, Chalk River data for $1 T^{\prime}-\mathrm{MoTe}_{2}$ phase. The initial purpose for this experiment was to map $1 T^{\prime}-\mathrm{MoTe}_{2}$ and $T_{d}-\mathrm{MoTe}_{2}$ coexistent region and phase transition, to confirm the x-ray powder diffraction structural determination results, and to identify the transition temperature. We wanted to study the structural changes through the coexistence region and determine how the crystal structure behaves in this region and how it changes from $1 T^{\prime}-\mathrm{MoTe}_{2}$ phase to $T_{d}-\mathrm{MoTe}_{2}$ phase. 

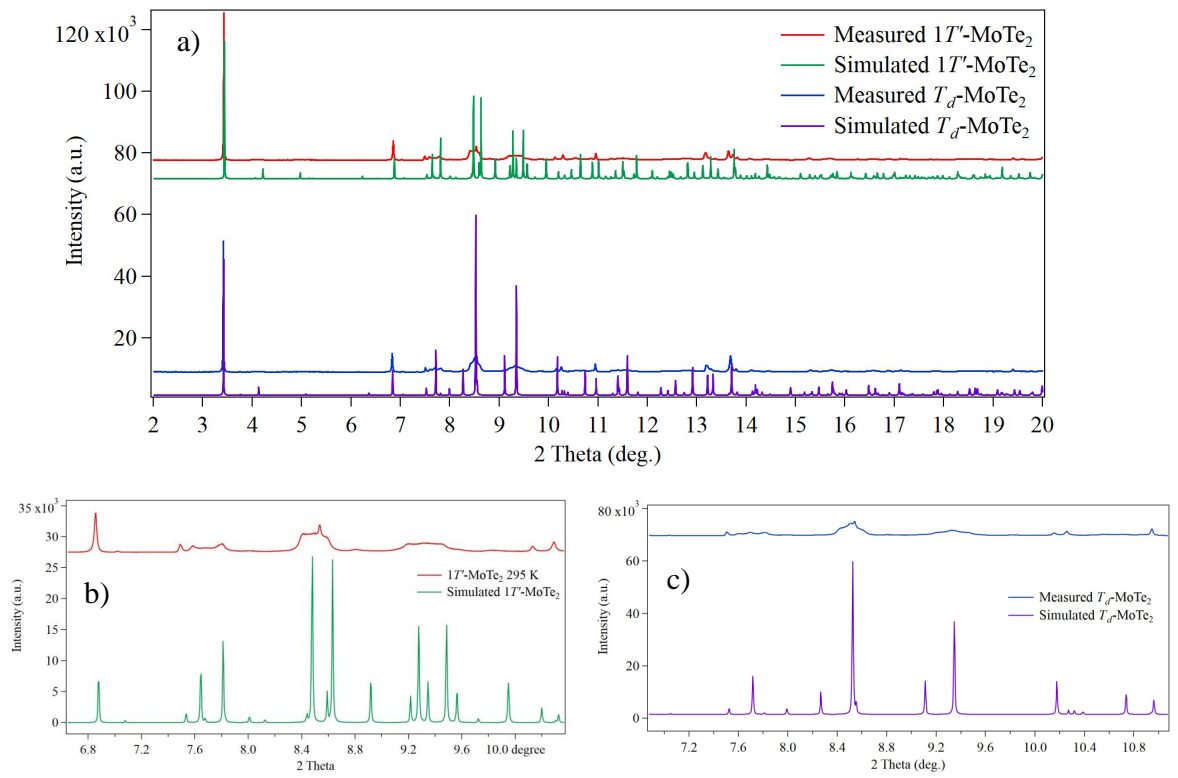

Figure 56. Comparison between X-ray powder diffraction patterns from APS experiment and simulated patterns using GSAS-II software. Panel a) shows a full range patterns for $1 T^{\prime}-\mathrm{MoTe}_{2}$ and $T_{d^{-}}-\mathrm{MoTe}_{2}$. A focused portion of the patterns are shown in b) and c) for phases $1 T^{\prime}-\mathrm{MoTe}_{2}$ and $T_{d}-\mathrm{MoTe}_{2}$, respectively.

Unfortunately, the neutron powder diffraction patterns was not reliable to perform a Rietveld analysis using GSAS-II. Figure 54 panel b at $64.8^{\circ}$ shows a peak that appear at $5 \mathrm{~K}$ and disappear at $240 \mathrm{~K}$ which might be evidence to the phase transition. It is worth mentioning that due to the low intensity in the neutron diffraction patterns, the changes in peaks that might represent a phase transition might not be visible. Since the coexistence area is not mapped very clear in the literature, we sought to determine its temperature range. Based on our data, we believe that the sample phase coexistence region is between $205 \mathrm{~K}$ and $240 \mathrm{~K}$.

A limitation of this study was the unintended low intensity neutron powder diffraction data which made it very difficult to analyze the data from CNBC using Rietveld analysis. Large single crystals are difficult to obtain by practically all growth methods. This makes the collection of single crystal diffraction data challenging. Nevertheless high-resolution single-crystalline data would be useful in understating the crystal model of $2 \mathrm{H}$ and $1 T^{\prime} \mathrm{MoTe}_{2}$. 


\subsection{Conclusion}

$\mathrm{MoTe}_{2}$ is an interesting material with very promising uses in the industry and physics theory. While $\mathrm{MoTe}_{2}$ maybe already in use for some applications, many applications utilized this material as thin films or few layers. Accurate analysis of bulk $\mathrm{MoTe}_{2}$ is difficult which has resulted in a limited understanding of the material. A probable explanation is that the $\mathrm{MoTe}_{2}$ a vdW material and its layers are loosely stacked. This makes it easy for the different layers to slide in, and therefor the atomic position alter, which makes it challenging for resolving the crystal structure. Also, the presence of vacancies was common in the studied phases of $\mathrm{MoTe}_{2}$. This indicates that atomic movements within the crystal structure is accessible to both Mo and Te atoms. Knowing the crystal structure and lattice parameters are important since they greatly effect the number and positions of Weyl points in the $T_{d}-\mathrm{MoTe}_{2}$ phase. Our current study provides a new detailed study of bulk $\mathrm{MoTe}_{2}$.

The main aim for this study was to characterize bulk $\mathrm{MoTe}_{2}$ phases. We performed measurements to check the quality of our samples and quantify the impurity level prior to diffraction experiments by using EDX and Raman spectroscopy. We employed on high resolution x-ray powder diffraction to analyze the crystal structure of $2 \mathrm{H}$ $\mathrm{MoTe}_{2}$ and $1 T^{\prime}-\mathrm{MoTe}_{2}$ phases at room and $100 \mathrm{~K}$. Our results were similar to what is reported in the literature. We identify the presence of vacancies specially at low temperature. These defects are related to the stacking faults in the $\mathrm{MoTe}_{2}$ material. We believe that its due to the weakly layer binding Van der Waals forces that allowed layers to lose with respect to other layers. We identified the coexistence region in $1 T^{\prime}-\mathrm{MoTe}_{2}$ phase to be between $205 \mathrm{~K}$ and $240 \mathrm{~K}$. 


\section{7 $\quad$ Further work}

Further study to investigate the local structure of $1 T^{\prime}-\mathrm{MoTe}_{2}$ as the phase changes to $T_{d}-\mathrm{MoTe}_{2}$ is needed. This will aid in understanding the phase transition and the coexistence region on the short scale.

The current work has initiated studies of the stacking faults and layer disordering

particularly in $1 T^{\prime}-\mathrm{MoTe}_{2}$. Studies on $\mathrm{MoTe}_{2}$ phases should investigate into details of twining of crystals and the stacking faults that might be present. The phase transition in $1 T^{\prime}-\mathrm{MoTe}_{2}$ can be used in many application such as pressure devices fabrication. 


\section{CHAPTER 8}

\section{CONCLUSIONS}

The current work was intended to provide a deeper understanding of the crystal structure of materials related to superconductivity. Superconducting materials are influential in the rapidly expanding field of condensed matter physics. The first aim of the present research was to investigate the crystal structural details of relatively new Mott insulator the $\mathrm{Ca}_{2} \mathrm{O}_{3} \mathrm{Fe}_{2.6} \mathrm{~S}_{2}$ and its doped variant $\mathrm{Ca}_{2} \mathrm{O}_{3} \mathrm{Fe}_{2.6} \mathrm{~S}_{1.75} \mathrm{Se}_{0.25}$. The magnetic structure of those materials was evaluated by estimating the order parameter through temperature dependent powder diffraction. The research's second aim

was to assess the short-range fluctuations in another Mott insulator, $\mathrm{La}_{2} \mathrm{O}_{2} \mathrm{Fe}_{2} \mathrm{OM}_{2}$ where $M=\mathrm{S}$, Se using the total neutron scattering and pair distribution function (PDF). The last aim of the study was to examine in detail the crystal structure of bulk $\mathrm{MoTe}_{2}$. The phases $2 \mathrm{H}-\mathrm{MoTe}_{2}$ and $1 T^{\prime}-\mathrm{MoTe}_{2}$ were studied using high-resolution synchrotron x-ray powder diffraction.

One of the main findings of this study was the presence of high iron atom vacancies in the pure and doped samples. Our results estimated the vacancies to reach approximately $30 \%$ of the total iron atomic sites. Such high vacancies $\mathrm{Ca}_{2} \mathrm{O}_{2} \mathrm{Fe}_{2.6} \mathrm{OS}_{2-x} \mathrm{Se}_{x}$ or similar materials will affect the quality of the diffraction pattern, electrical transport properties, and the magnetic structure. Another important finding was the similarity between $\mathrm{Ca}_{2} \mathrm{O}_{2} \mathrm{Fe}_{2.6} \mathrm{OS}_{2-x} \mathrm{Se}_{x}$ and $\mathrm{La}_{2} \mathrm{O}_{2} \mathrm{Fe}_{2} \mathrm{O}(\mathrm{S}, \mathrm{Se})_{2}$ based on the critical $\beta$ value. Our temperature-dependent neutron powder diffraction revealed that $\mathrm{Ca}_{2} \mathrm{O}_{3} \mathrm{Fe}_{2.6} \mathrm{~S}_{2}$ has the critical exponent $\beta$ of 0.1228 . This $\beta$ value implies that this Mott insulator 
is similar to $\mathrm{La}_{2} \mathrm{O}_{3} \mathrm{Fe}_{2} \mathrm{M}_{2}$, which has a comparable critical exponent value and thus can be associated with the two dimensional antiferromagnetic Ising model.

The short-range structural study on $\mathrm{La}_{2} \mathrm{O}_{2} \mathrm{Fe}_{2} \mathrm{OM}_{2}$ for $M=\mathrm{S}$ or Se, showed the strong presence of orthorhombic distortion on the short scale while the average scale maintained its tetragonal symmetry. These structural distortions are strong evidence of nematic behavior on the short scale in these Mott insulators. The study shows strong evidence of short-range symmetry breaking in iron-based materials. This behavior of the Mott insulator is similar to iron pnictides superconductors.

The discovery of graphene laid the ground for diverse applications based on 2D material. Naturally, closer attention was given to other materials that exhibit similar properties as graphene. One of those materials was the transition metal dichalcogenide $\mathrm{MoTe}_{2}$ which has three stable phases. In the $2 \mathrm{H}-\mathrm{MoTe}_{2}$ phase that we studied, vacancies were found at room-temperature and increased at low temperatures. The presence of these vacancies made it very difficult to evaluate the structure through Rietveld analysis. While not confirmed by this study, we believe that the presence of these vacancies affects the transport measurements. $\mathrm{MoTe}_{2}$ is a topological superconductor and a type-II Weyl semimetal. Understanding the details of the $\mathrm{MoTe}_{2}$ crystal structure will help in determining the positions of the Weyl points (WP). $1 T^{\prime}$ $\mathrm{MoTe}_{2}$ phase transition is also estimated based on temperature dependence powder diffraction to be between $200 \mathrm{~K}$ and $240 \mathrm{~K}$.

Detailed analysis of crystal structure is a significant step when determining the properties of the studied material. It gives information about the connectivity, accurate bond lengths and angles, and three-dimensional packing of the specific material. Based on the precision information of the crystal structure density functional theory (DFT) calculation can be made, magnetic structure analysis can be determined, and the quality of the crystal can be verified. The total neutron scattering and PDF analysis are powerful tools to investigate the short-range symmetry in materials. The 
work done in this research can be beneficial for further studies of TMDs, iron-based superconductors, and Mott insulators. 


\section{REFERENCES}

[1] J. Paglione and R. L. Greene, "High-temperature superconductivity in ironbased materials," Nature physics 6, 645 (2010).

[2] G. Kotliar and D. Vollhardt, "Strongly correlated materials: Insights from dynamical mean-field theory," Physics today 57, 53-60 (2004).

[3] M. Xu, T. Liang, M. Shi, and H. Chen, "Graphene-like two-dimensional materials," Chemical reviews 113, 3766-3798 (2013).

[4] Y. Sun, S.-C. Wu, M. N. Ali, C. Felser, and B. Yan, "Prediction of weyl semimetal in orthorhombic mote 2," Physical Review B 92, 161107 (2015).

[5] E. Kisi and C. Howard, Applications of Neutron Powder Diffraction, Oxford Series on Neutron Scattering in Condensed Matter (OUP Oxford, 2012).

[6] G. E. Bacon, "Neutron diffraction," (1955).

[7] Y. Zhou and S. Ramanathan, "Mott memory and neuromorphic devices," Proceedings of the IEEE 103, 1289-1310 (2015).

[8] C. Kittel et al., Introduction to solid state physics, vol. 8 (Wiley New York, 1976).

[9] B. Freelon, Z. Yamani, I. Swainson, R. Flacau, B. Karki, Y. H. Liu, L. Craco, M. S. Laad, M. Wang, J. Chen, R. J. Birgeneau, and M. Fang, "Magnetic and structural properties of the iron oxychalcogenides $\mathrm{La}_{2} \mathrm{O}_{2} \mathrm{Fe}_{2} \mathrm{OM}_{2}, M=(\mathrm{S}, \mathrm{Se})$," Phys. Rev. B 99, 024109 (2019).

[10] H. Kabbour, E. Janod, B. Corraze, M. Danot, C. Lee, M.-H. Whangbo, and L. Cario, "Structure and magnetic properties of oxychalcogenides $\mathrm{A}_{2} \mathrm{~F}_{2} \mathrm{Fe}_{2} \mathrm{OQ}_{2}$ $(\mathrm{A}=\mathrm{Sr}, \mathrm{Ba} ; \mathrm{Q}=\mathrm{S}, \mathrm{Se})$ with $\mathrm{Fe}_{2} \mathrm{O}$ square planar layers representing an antiferromagnetic checkerboard spin lattice," Journal of the American Chemical Society 130, 8261-8270 (2008).

[11] H. Zhang, X. Wu, D. Li, S. Jin, X. Chen, T. Zhang, Z. Lin, S. Shen, D. Yuan, and $\mathrm{X}$. Chen, " $\mathrm{Ca}_{2} \mathrm{O}_{3} \mathrm{Fe}_{2.6} \mathrm{~S}_{2}$ : an antiferromagnetic Mott insulator at proximity to bad metal," Journal of Physics: Condensed Matter 28, 145701 (2016).

[12] E. Abrahams and Q. Si, "Quantum criticality in the iron pnictides and chalcogenides," Journal of physics: Condensed matter 23, 223201 (2011).

[13] J.-X. Zhu, R. Yu, H. Wang, L. L. Zhao, M. D. Jones, J. Dai, E. Abrahams, E. Morosan, M. Fang, and Q. Si, "Band narrowing and Mott localization in iron oxychalcogenides $\mathrm{La}_{2} \mathrm{O}_{2} \mathrm{Fe}_{2} \mathrm{O}(\mathrm{Se}, \mathrm{S})_{2}$," Phys. Rev. Lett. 104, 216405 (2010). 
[14] C. Stock and E. E. McCabe, "The magnetic and electronic properties of oxyselenides influence of transition metal ions and lanthanides," Journal of Physics: Condensed Matter 28, 453001 (2016).

[15] N. Ni, E. Climent-Pascual, S. Jia, Q. Huang, and R. J. Cava, "Physical properties and magnetic structure of the layered oxyselenide $\mathrm{La}_{2} \mathrm{O}_{3} \mathrm{Mn}_{2} \mathrm{Se}_{2}$," Physical Review B 82, 214419 (2010).

[16] B. Karki, A. Alfailakawi, B. Frandsen, M. Everett, J. Neuefeind, B. Xu, H. Wang, M. Fang, and B. Freelon, "Local Structure of Mott Insulating Iron Oxychalcogenides $\mathrm{La}_{2} \mathrm{O}_{2} \mathrm{Fe}_{2} \mathrm{OM}_{2}(M=\mathrm{S}, \mathrm{Se})$," arXiv preprint arXiv:2002.10305 (2020).

[17] R. M. Fernandes, A. V. Chubukov, and J. Schmalian, "What drives nematic order in iron-based superconductors?" Nature physics 10, 97 (2014).

[18] E. Dagotto, "Complexity in strongly correlated electronic systems," Science 309, 257-262 (2005).

[19] P. Dai, J. Hu, and E. Dagotto, "Magnetism and its microscopic origin in ironbased high-temperature superconductors," Nature Physics 8, 709 (2012).

[20] J. Li, P. J. Pereira, J. Yuan, Y.-Y. Lv, M.-P. Jiang, D. Lu, Z.-Q. Lin, Y.-J. Liu, J.-F. Wang, L. Li et al., "Nematic superconducting state in iron pnictide superconductors," Nature communications 8, 1880 (2017).

[21] L. Zhou, K. Xu, A. Zubair, A. D. Liao, W. Fang, F. Ouyang, Y.-H. Lee, K. Ueno, R. Saito, T. Palacios et al., "Large-area synthesis of high-quality uniform fewlayer $\mathrm{MoTe}_{2}$," Journal of the American Chemical Society 137, 11892-11895 (2015).

[22] A. Chubukov and P. J. Hirschfeld, "Fe-based superconductors: seven years later," Physics today 68, 46 (2015).

[23] I. I. Mazin, "Superconductivity gets an iron boost," Nature 464, 183 (2010).

[24] A. Farhoodfar, R. Gooding, and W. Atkinson, "Variational Monte Carlo study of Anderson localization in the Hubbard model," Physical Review B 84, 205125 (2011).

[25] R. T. Scalettar, "An introduction to the Hubbard Hamiltonian," Quantum Materials: Experiments and Theory 6 (2016).

[26] Y. Shiohara, M. Yoshizumi, Y. Takagi, and T. Izumi, "Future prospects of high $\mathrm{T}_{c}$ superconductors-coated conductors and their applications," Physica C: Superconductivity 484, 1-5 (2013).

[27] M. Fischer, "Superconducting SeaTitan opens up a new path to 10 MW," Renewable Energy World 12 (2010). 
[28] Y. Song, Z. Yamani, C. Cao, Y. Li, C. Zhang, J. S. Chen, Q. Huang, H. Wu, J. Tao, Y. Zhu et al., "A Mott insulator continuously connected to iron pnictide superconductors," Nature communications 7, 1-8 (2016).

[29] S. Raghu, X.-L. Qi, C. Honerkamp, and S.-C. Zhang, "Topological Mott insulators," Physical review letters 100, 156401 (2008).

[30] D. M. Newns, "Junction mott transition field effect transistor (JMTFET) and switch for logic and memory applications," (2000). US Patent 6,121,642.

[31] J. Son, S. Rajan, S. Stemmer, and S. James Allen, "A heterojunction modulation-doped mott transistor," Journal of Applied Physics 110, 084503 (2011).

[32] Y. Zhou and S. Ramanathan, "Correlated electron materials and field effect transistors for logic: a review," Critical Reviews in Solid State and Materials Sciences 38, 286-317 (2013).

[33] B. A. Frandsen, L. Liu, S. C. Cheung, Z. Guguchia, R. Khasanov, E. Morenzoni, T. J. Munsie, A. M. Hallas, M. N. Wilson, Y. Cai et al., "Volume-wise destruction of the antiferromagnetic Mott insulating state through quantum tuning," Nature communications 7, 12519 (2016).

[34] E. Morosan, D. Natelson, A. H. Nevidomskyy, and Q. Si, "Strongly correlated materials," Advanced Materials 24, 4896-4923 (2012).

[35] C. Giannetti, M. Capone, D. Fausti, M. Fabrizio, F. Parmigiani, and D. Mihailovic, "Ultrafast optical spectroscopy of strongly correlated materials and high-temperature superconductors: a non-equilibrium approach," Advances in Physics 65, 58-238 (2016).

[36] B. Coles, A. Hillier, F. Coomer, N. Bristowe, S. Ramos, and E. McCabe, "Spin interactions and magnetic order in the iron oxychalcogenides $\mathrm{BaFe}_{2} \mathrm{Q}_{2} \mathrm{O}$," arXiv preprint arXiv:1902.09035 (2019).

[37] A. V. Chubukov, R. M. Fernandes, and J. Schmalian, "Origin of nematic order in FeSe," Physical Review B 91, 201105 (2015).

[38] Y. Kushnirenko, D. Evtushinsky, T. Kim, I. Morozov, L. Harnagea, S. Wurmehl, S. Aswartham, A. Chubukov, and S. Borisenko, "Superconductivity-induced nematicity," arXiv preprint arXiv:1810.04446 (2018).

[39] J.-H. Chu, H.-H. Kuo, J. G. Analytis, and I. R. Fisher, "Divergent nematic susceptibility in an iron arsenide superconductor," Science 337, 710-712 (2012).

[40] F. Koppens, T. Mueller, P. Avouris, A. Ferrari, M. Vitiello, and M. Polini, "Photodetectors based on graphene, other two-dimensional materials and hybrid systems," Nature nanotechnology 9, 780 (2014). 
[41] N. Armitage, E. Mele, and A. Vishwanath, "Weyl and Dirac semimetals in three-dimensional solids," Reviews of Modern Physics 90, 015001 (2018).

[42] H. Weyl, "Gravitation and the electron," Proceedings of the National Academy of Sciences of the United States of America 15, 323 (1929).

[43] C. Wang, L. Gioia, and A. Burkov, "Fractional Quantum Hall Effect in Weyl Semimetals," arXiv preprint arXiv:1907.02068 (2019).

[44] T. M. McCormick, R. C. McKay, and N. Trivedi, "Semiclassical theory of anomalous transport in type-II topological Weyl semimetals," Physical Review B 96, 235116 (2017).

[45] R. Lundgren, P. Laurell, and G. A. Fiete, "Thermoelectric properties of Weyl and Dirac semimetals," Physical Review B 90, 165115 (2014).

[46] H.-J. Kim, K.-S. Kim, J.-F. Wang, M. Sasaki, N. Satoh, A. Ohnishi, M. Kitaura, M. Yang, and L. Li, "Dirac versus Weyl fermions in topological insulators: Adler-Bell-Jackiw anomaly in transport phenomena," Physical review letters 111, 246603 (2013).

[47] M. Koshino and I. F. Hizbullah, "Magnetic susceptibility in three-dimensional nodal semimetals," Physical Review B 93, 045201 (2016).

[48] A. Altland and D. Bagrets, "Effective field theory of the disordered Weyl semimetal," Physical review letters 114, 257201 (2015).

[49] G. Fiori, F. Bonaccorso, G. Iannaccone, T. Palacios, D. Neumaier, A. Seabaugh, S. K. Banerjee, and L. Colombo, "Electronics based on two-dimensional materials," Nature nanotechnology 9, 768 (2014).

[50] M. Lang, L. He, F. Xiu, X. Yu, J. Tang, Y. Wang, X. Kou, W. Jiang, A. V. Fedorov, and K. L. Wang, "Revelation of topological surface states in $\mathrm{Bi}_{2} \mathrm{Se}_{3}$ thin films by in-situ Al passivation," Acs Nano 6, 295-302 (2011).

[51] F. Gamble and B. Silbernagel, "Anisotropy of the proton spin-lattice relaxation time in the superconducting intercalation complex $\mathrm{TaS}_{2}\left(\mathrm{NH}_{3}\right)$ : Structural and bonding implications," The Journal of Chemical Physics 63, 2544-2552 (1975).

[52] X. Tang, W. Xie, H. Li, W. Zhao, Q. Zhang, and M. Niino, "Preparation and thermoelectric transport properties of high-performance p-type $\mathrm{Bi}_{2} \mathrm{Te}_{3}$ with layered nanostructure," Applied physics letters 90, 012102 (2007).

[53] T. Shishidou, A. J. Freeman, and R. Asahi, "Effect of GGA on the halfmetallicity of the itinerant ferromagnet $\mathrm{CoS}_{2}$," Physical Review B 64, 180401 (2001).

[54] K. Takada, H. Sakurai, E. Takayama-Muromachi, F. Izumi, R. A. Dilanian, and T. Sasaki, "Superconductivity in two-dimensional $\mathrm{CoO}_{2}$ layers," Nature 422, $53(2003)$. 
[55] R. Withers and J. Wilson, "An examination of the formation and characteristics of charge-density waves in inorganic materials with special reference to the two-and one-dimensional transition-metal chalcogenides," Journal of Physics C: Solid State Physics 19, 4809 (1986).

[56] F. Zhang, H. Zhang, P. Shrestha, Y. Zhu, K. Maize, S. Krylyuk, A. Shakouri, J. Campbell, K. Cheung, L. Bendersky et al., "An ultra-fast multi-level mote 2based rram," in "2018 IEEE International Electron Devices Meeting (IEDM)," (IEEE, 2018), pp. 22-7.

[57] W. S. Yun, S. Han, S. C. Hong, I. G. Kim, and J. Lee, "Thickness and strain effects on electronic structures of transition metal dichalcogenides: $2 \mathrm{H}_{-} \mathrm{MX}_{2}$ semiconductors $(\mathrm{M}=\mathrm{Mo}, \mathrm{W} ; \mathrm{X}=\mathrm{S}, \mathrm{Se}, \mathrm{Te})$," Physical Review B 85, 033305 (2012).

[58] J. Als-Nielsen and D. McMorrow, Elements of Modern X-ray Physics (Wiley, 2011).

[59] A. Bail, I. Madsen, L. Cranswick, J. Cockcroft, P. Norby, A. Zuev, A. Fitch, J. Rodriguez-Carvajal, C. Giacovazzo, R. Von Dreele et al., Powder Diffraction: Theory and Practice (Royal Society of Chemistry, 2015).

[60] J. Wang, B. H. Toby, P. L. Lee, L. Ribaud, S. M. Antao, C. Kurtz, M. Ramanathan, R. B. Von Dreele, and M. A. Beno, "A dedicated powder diffraction beamline at the advanced photon source: Commissioning and early operational results," Review of Scientific Instruments 79, 085105 (2008).

[61] J. M. Carpenter, "Pulsed spallation neutron sources for slow neutron scattering," Nuclear Instruments and Methods 145, 91-113 (1977).

[62] G. L. Squires, "Introduction to the theory of thermal neutron scattering," Physics Today 32, 69 (1979).

[63] A. Huq, J. P. Hodges, O. Gourdon, and L. Heroux, "Powgen: A third-generation high-resolution high-throughput powder diffraction instrument at the Spallation Neutron Source," Z. Kristallogr. Proc 1, 127-135 (2011).

[64] J. Neuefeind, M. Feygenson, J. Carruth, R. Hoffmann, and K. K. Chipley, "The Nanoscale Ordered MAterials Diffractometer NOMAD at the Spallation Neutron Source SNS," Nuclear Instruments and Methods in Physics Research Section B: Beam Interactions with Materials and Atoms 287, 68 - 75 (2012).

[65] H. Rietveld, "Line profiles of neutron powder-diffraction peaks for structure refinement," Acta Crystallographica 22, 151-152 (1967).

[66] H. Rietveld, "A profile refinement method for nuclear and magnetic structures," Journal of applied Crystallography 2, 65-71 (1969). 
[67] T. Egami and S. J. L. Billinge, Underneath the Bragg Peaks: Structural Analysis of Complex Materials (Pergamon Press Elsevier, Oxford, England, 2003).

[68] S. J. Billinge, "The rise of the x-ray atomic pair distribution function method: a series of fortunate events," Philosophical Transactions of the Royal Society A 377, 20180413 (2019).

[69] P. Scardi and L. Gelisio, "Vibrational properties of nanocrystals from the debye scattering equation," Scientific reports 6, 22221 (2016).

[70] T. Proffen and S. Billinge, "PDFFIT, a program for full profile structural refinement of the atomic pair distribution function," Journal of Applied Crystallography 32, 572-575 (1999).

[71] C. L. Farrow, P. Juhas, J. W. Liu, D. Bryndin, E. S. Božin, J. Bloch, T. Proffen, and S. J. L. Billinge, "PDFfit2 and PDFgui: computer programs for studying nanostructure in crystals," Journal of Physics: Condensed Matter 19, 335219 (2007).

[72] P. Juhás, J. N. Louwen, L. van Eijck, E. T. C. Vogt, and S. J. L. Billinge, "PDFgetN3: atomic pair distribution functions from neutron powder diffraction data using ad hoc corrections," Journal of Applied Crystallography 51, 1492 (2018).

[73] Y. Werman and E. Berg, "Mott-Ioffe-Regel limit and resistivity crossover in a tractable electron-phonon model," Physical Review B 93, 075109 (2016).

[74] H. H. Ma, P. Yang, Z. Lim et al., "Mott variable range hopping and bad-metal in lightly doped spin-orbit Mott insulator $\mathrm{BaIrO}_{3}$," Physical Review Materials 2, 065003 (2018).

[75] N. Mott, "The effect of electron interaction on variable-range hopping," The Philosophical Magazine: A Journal of Theoretical Experimental and Applied Physics 34, 643-645 (1976).

[76] J.-K. Bao, C.-M. Feng, Y.-K. Luo, H. Jiang, Y.-L. Sun, W.-H. Jiao, C.-Y. Shen, Z.-A. Xu, and G.-H. Cao, "Variable range hopping conductivity and spin glass behavior in spin-ladder ba0. 6k0. 4fe2se3 single crystals," Journal of Physics: Condensed Matter 26, 026002 (2013).

[77] J. He, D. Wang, H. Shi, H. Yang, J. Li, and G. Chen, "Synthesis, structure, and magnetic properties of the layered iron oxychalcogenide na 2 fe 2 se 2 o," Physical Review B 84, 205212 (2011).

[78] P. A. Lee, N. Nagaosa, and X.-G. Wen, "Doping a mott insulator: Physics of high-temperature superconductivity," Reviews of modern physics 78, 17 (2006).

[79] S. Landsgesell, K. Prokeš, T. Hansen, and M. Frontzek, "An unexpected gap: Magnetic structures of la2o3 (fe1-xmnx) 2se2 investigated by neutron diffraction and physical property measurements," Acta materialia 66, 232-240 (2014). 
[80] N. W. Ashcroft, N. D. Mermin et al., Solid state physics (New York: Holt, Rinehart and Winston, 1976).

[81] L. Craco, B. Freelon, A. Alafailakawi, B. Karki, and S. Leoni, "Site-selective electronic structure of pure and doped $\mathrm{Ca}_{2} \mathrm{O}_{3} \mathrm{Fe}_{3} \mathrm{~S}_{2}$," Physical Review B 98, 045130 (2018).

[82] M. Guidry, Y. Sun, L.-A. Wu, and C.-L. Wu, "Fermion dynamical symmetry and strongly-correlated electrons: a comprehensive model of high-temperature superconductivity," arXiv preprint arXiv:2003.07994 (2020).

[83] W. Ruan, "High temperature superconductivity: Cooper pairs in trap," arXiv preprint arXiv:1306.3547 (2013).

[84] O. Arnold, J.-C. Bilheux, J. Borreguero, A. Buts, S. I. Campbell, L. Chapon, M. Doucet, N. Draper, R. F. Leal, M. Gigg et al., "Mantid-data analysis and visualization package for neutron scattering and $\mu$ sr experiments," Nuclear Instruments and Methods in Physics Research Section A: Accelerators, Spectrometers, Detectors and Associated Equipment 764, 156-166 (2014).

[85] B. H. Toby and R. B. Von Dreele, "GSAS-II: the genesis of a modern opensource all purpose crystallography software package," Journal of Applied Crystallography 46, 544-549 (2013).

[86] G. Pawley, "Unit-cell refinement from powder diffraction scans," Journal of Applied Crystallography 14, 357-361 (1981).

[87] Z. Zhou, W. Jin, W. Li, S. Nandi, B. Ouladdiaf, Z. Yan, X. Wei, X. Xu, W. Jiao, N. Qureshi et al., "Universal critical behavior in the ferromagnetic superconductor eu (fe 0.75 ru 0.25) 2 as 2," Physical Review B 100, 060406 (2019).

[88] Q. Si, R. Yu, and E. Abrahams, "High-temperature superconductivity in iron pnictides and chalcogenides," Nature Reviews Materials 1, 16017 (2016).

[89] J. Paglione and R. L. Greene, "High-temperature superconductivity in ironbased materials," Nature Physics 6, 645 (2010).

[90] M. Yi, Y. Zhang, Z.-X. Shen, and D. Lu, "Role of the orbital degree of freedom in iron-based superconductors," npj Quantum Materials 2, 1-12 (2017).

[91] R. M. Fernandes, A. V. Chubukov, and J. Schmalian, "What drives nematic order in iron-based superconductors?" Nature Physics 10, 97 (2014).

[92] P. A. Lee, N. Nagaosa, and X.-G. Wen, "Doping a Mott insulator: Physics of high-temperature superconductivity," Rev. Mod. Phys. 78, 17-85 (2006).

[93] I. Mazin, S. A. Kimber, and D. N. Argyriou, "Quasiparticle interference in antiferromagnetic parent compounds of iron-based superconductors," Physical Review B 83, 052501 (2011). 
[94] D. C. Johnston, "The puzzle of high temperature superconductivity in layered iron pnictides and chalcogenides," Advances in Physics 59, 803-1061 (2010).

[95] E. E. McCabe, C. Stock, E. E. Rodriguez, A. S. Wills, J. W. Taylor, and J. S. O. Evans, "Weak spin interactions in Mott insulating $\mathrm{La}_{2} \mathrm{O}_{2} \mathrm{Fe}_{2} \mathrm{OSe}_{2}$," Phys. Rev. B 89, 100402 (2014).

[96] J. M. Mayer, L. F. Schneemeyer, T. Siegrist, J. V. Waszczak, and B. Van Dover, "New layered iron-lanthanum-oxide-sulfide and -selenide phases: $\mathrm{Fe}_{2} \mathrm{La}_{2} \mathrm{O}_{3} \mathrm{E}_{2}$ $(\mathrm{E}=\mathrm{S}, \mathrm{Se}), "$ Angewandte Chemie International Edition in English 31, 16451647 (1992).

[97] J. B. He, D. M. Wang, H. L. Shi, H. X. Yang, J. Q. Li, and G. F. Chen, "Synthesis, structure, and magnetic properties of the layered iron oxychalcogenide $\mathrm{Na}_{2} \mathrm{Fe}_{2} \mathrm{Se}_{2} \mathrm{O}, "$ Phys. Rev. B 84, 205212 (2011).

[98] R. K. Oogarah, E. Suard, and E. E. McCabe, "Magnetic order and phase transition in the iron oxysulfide $\mathrm{La}_{2} \mathrm{O}_{2} \mathrm{Fe}_{2} \mathrm{OS}_{2}$," Journal of Magnetism and Magnetic Materials 446, 101-107 (2018).

[99] D. G. Free and J. S. O. Evans, "Low-temperature nuclear and magnetic structures of $\mathrm{La}_{2} \mathrm{O}_{2} \mathrm{Fe}_{2} \mathrm{OSe}_{2}$ from x-ray and neutron diffraction measurements," Phys. Rev. B 81, 214433 (2010).

[100] R. J. Koch, T. Konstantinova, M. Abeykoon, A. Wang, C. Petrovic, Y. Zhu, E. S. Bozin, and S. J. L. Billinge, "Room temperature local nematicity in FeSe superconductor," Phys. Rev. B 100, 020501 (2019).

[101] B. A. Frandsen, K. M. Taddei, D. E. Bugaris, R. Stadel, M. Yi, A. Acharya, R. Osborn, S. Rosenkranz, O. Chmaissem, and R. J. Birgeneau, "Widespread orthorhombic fluctuations in the ( $\mathrm{Sr}, \mathrm{Na}) \mathrm{Fe}_{2} \mathrm{As}_{2}$ family of superconductors," Phys. Rev. B 98, 180505 (2018).

[102] C. E. White, J. L. Provis, T. Proffen, and J. S. Van Deventer, "The effects of temperature on the local structure of metakaolin-based geopolymer binder: A neutron pair distribution function investigation," Journal of the American Ceramic Society 93, 3486-3492 (2010).

[103] M. Paściak, P. Ondrejkovic, J. Kulda, P. Vaněk, J. Drahokoupil, G. Steciuk, L. Palatinus, T. Welberry, H. Fischer, J. Hlinka et al., "Local structure of relaxor ferroelectric sr $\mathrm{x}$ ba $1-\mathrm{x}$ nb 2 o 6 from a pair distribution function analysis," Physical Review B 99, 104102 (2019).

[104] W. A. Slawinski, H. Y. Playford, S. Hull, S. T. Norberg, S. G. Eriksson, T. Gustafsson, K. Edstrom, and W. R. Brant, "Neutron pair distribution function study of fepo4 and lifepo4." Chemistry of Materials 31, 5024 (2019). 
[105] I.-K. Jeong and B.-G. Kim, "High-temperature neutron powder diffraction studies on atomic structure of (1- x) pbtio 3 -x bi (zn 0.5 ti 0.5 ) o 3," Journal of Applied Physics 126, 014101 (2019).

[106] A. Masadeh, E. Božin, C. Farrow, G. Paglia, P. Juhas, S. Billinge, A. Karkamkar, and M. G. Kanatzidis, "Quantitative size-dependent structure and strain determination of CdSe nanoparticles using atomic pair distribution function analysis," Physical Review B 76, 115413 (2007).

[107] B. Toby and T. Egami, "Accuracy of pair distribution function analysis applied to crystalline and non-crystalline materials," Acta Crystallographica Section A: Foundations of Crystallography 48, 336-346 (1992).

[108] C. Farrow, P. Juhas, J. Liu, D. Bryndin, E. Božin, J. Bloch, T. Proffen, and S. Billinge, "Pdffit2 and pdfgui: computer programs for studying nanostructure in crystals," Journal of Physics: Condensed Matter 19, 335219 (2007).

[109] A. M. Abeykoon, W. Donner, M. Brunelli, M. Castro-Colin, A. J. Jacobson, and S. C. Moss, "From Average to Local Structure: A Rietveld and an Atomic Pair Distribution Function (PDF) Study of Selenium Clusters in Zeolite-NdY," Journal of the American Chemical Society 131, 13230-13231 (2009).

[110] K. Horigane, K. Kawashima, S. Ji, M. Yoshikawa, D. Louca, and J. Akimitsu, "Local crystal structure of Mott-insulating iron oxychalcogenides $\mathrm{La}_{2} \mathrm{O}_{2} \mathrm{Fe}_{2} \mathrm{OSe}_{2}$," in "Proceedings of the International Conference on Strongly Correlated Electron Systems (SCES2013)," (2014), p. 015039.

[111] K. Horigane, A. Llobet, and D. Louca, "Suppression of magnetic coupling by in-plane buckling in $\mathrm{SrFeO}_{2}, "$ Phys. Rev. Lett. 112, 097001 (2014).

[112] E. Fradkin, S. A. Kivelson, M. J. Lawler, J. P. Eisenstein, and A. P. Mackenzie, "Nematic Fermi fluids in condensed matter physics," Annu. Rev. Condens. Matter Phys. 1, 153-178 (2010).

[113] H.-H. Kuo, J.-H. Chu, J. C. Palmstrom, S. A. Kivelson, and I. R. Fisher, "Ubiquitous signatures of nematic quantum criticality in optimally doped Febased superconductors," Science 352, 958-962 (2016).

[114] A. E. Böhmer and C. Meingast, "Electronic nematic susceptibility of iron-based superconductors," Comptes Rendus Physique 17, 90 - 112 (2016).

[115] E. P. Rosenthal, E. F. Andrade, C. J. Arguello, R. M. Fernandes, L. Y. Xing, X. C. Wang, C. Q. Jin, A. J. Millis, and A. N. Pasupathy, "Visualization of electron nematicity and unidirectional antiferroic fluctuations at high temperatures in NaFeAs," Nature Physics 10, 225 (2014).

[116] B. A. Frandsen, Q. Wang, S. Wu, J. Zhao, and R. J. Birgeneau, "Quantitative characterization of short-range orthorhombic fluctuations in FeSe through pair distribution function analysis," Phys. Rev. B 100, 020504 (2019). 
[117] J. Wang, G.-Z. Liu, D. V. Efremov, and J. van den Brink, "Order parameter fluctuation and ordering competition in $\mathrm{Ba}_{1-x} \mathrm{~K}_{x} \mathrm{Fe}_{2} \mathrm{As}_{2}$," Phys. Rev. B 95, 024511 (2017).

[118] P. Dai, "Antiferromagnetic order and spin dynamics in iron-based superconductors," Rev. Mod. Phys. 87, 855-896 (2015).

[119] G. R. Stewart, "Superconductivity in iron compounds," Rev. Mod. Phys. 83, 1589-1652 (2011).

[120] D. Jariwala, V. K. Sangwan, L. J. Lauhon, T. J. Marks, and M. C. Hersam, "Emerging device applications for semiconducting two-dimensional transition metal dichalcogenides," ACS Nano 8, 1102-1120 (2014).

[121] Y.-M. Chang, C.-Y. Lin, Y.-F. Lin, and K. Tsukagoshi, "Two-dimensional $\mathrm{MoTe}_{2}$ materials: From synthesis, identification, and charge transport to electronics applications," Japanese Journal of Applied Physics 55, 1102A1 (2016).

[122] W. Yu, S. Li, Y. Zhang, W. Ma, T. Sun, J. Yuan, K. Fu, and Q. Bao, "Nearinfrared photodetectors based on $\mathrm{MoTe}_{2} /$ graphene heterostructure with high responsivity and flexibility," Small 13, 1700268-n/a (2017).

[123] H.-J. Kim, S.-H. Kang, I. Hamada, and Y.-W. Son, "Origins of the structural phase transitions in $M o T e_{2}$ and $W T e_{2}, "$ Physical Review B 95, 180101 (2017).

[124] D. Puotinen and R. E. Newnham, "The crystal structure of $\mathrm{MoTe}_{2}$," Acta Crystallographica 14, 691-692 (1961).

[125] D. H. Keum, S. Cho, J. H. Kim, D.-H. Choe, H.-J. Sung, M. Kan, H. Kang, J.Y. Hwang, S. W. Kim, H. Yang, K. J. Chang, and Y. H. Lee, "Bandgap opening in few-layered monoclinic MoTe 2 ," Nature Physics 11, 482 EP - (2015).

[126] X.-M. Zhao, H.-y. Liu, A. F. Goncharov, Z.-W. Zhao, V. V. Struzhkin, H.K. Mao, A. G. Gavriliuk, and X.-J. Chen, "Pressure effect on the electronic, structural, and vibrational properties of layered $2 \mathrm{H}-\mathrm{MoTe}_{2}, "$ Physical Review B 99, 024111 (2019).

[127] T. J. S. Anand, C. Sanjeeviraja, and M. Jayachandran, "Preparation of layered semiconductor $\left(\mathrm{MoSe}_{2}\right)$ by electrosynthesis," Vacuum 60, 431-435 (2001).

[128] R. Adler, M. Flanigan, J. Huang, R. Kling, N. Kushalnagar, L. Nachman, C.-Y. Wan, and M. Yarvis, "Intel mote 2: an advanced platform for demanding sensor network applications," in "Proceedings of the 3rd international conference on Embedded networked sensor systems," (2005), pp. 298-298.

[129] J. Yang, T. Lu, Y. W. Myint, J. Pei, D. Macdonald, J.-C. Zheng, and Y. Lu, "Robust excitons and trions in monolayer $\mathrm{MoTe}_{2}$," ACS nano 9, 6603-6609 (2015). 
[130] H. S. Lee, K. Choi, J. S. Kim, S. Yu, K. R. Ko, and S. Im, "Coupling TwoDimensional $\mathrm{MoTe}_{2}$ and InGaZnO Thin-Film Materials for Hybrid PN Junction and CMOS Inverters," ACS Applied Materials \& Interfaces 9, 15592-15598 (2017).

[131] Z. Wang, F. Wang, L. Yin, Y. Huang, K. Xu, F. Wang, X. Zhan, and J. He, "Electrostatically tunable lateral $\mathrm{MoTe}_{2}$ p-n junction for use in highperformance optoelectronics," Nanoscale 8, 13245-13250 (2016).

[132] H. Ji, M.-K. Joo, H. Yi, H. Choi, H. Z. Gul, M. K. Ghimire, and S. C. Lim, "Tunable Mobility in Double-Gated $\mathrm{MoTe}_{2}$ Field-Effect Transistor: Effect of Coulomb Screening and Trap Sites," ACS Applied Materials \& Interfaces 9, 29185-29192 (2017).

[133] J. Y. Lim, A. Pezeshki, S. Oh, J. S. Kim, Y. T. Lee, S. Yu, D. K. Hwang, G.-H. Lee, H. J. Choi, and S. Im, "Homogeneous 2D MoTe 2 p-n Junctions and CMOS Inverters formed by Atomic-Layer-Deposition-Induced Doping," Advanced Materials 29, 1701798 (2017).

[134] S. Nakaharai, M. Yamamoto, K. Ueno, and K. Tsukagoshi, "Carrier polarity control in $\alpha$-mote2 schottky junctions based on weak fermi-level pinning," ACS Applied Materials \& Interfaces 8, 14732-14739 (2016).

[135] Z. Feng, Y. Xie, E. Wu, Y. Yu, S. Zheng, R. Zhang, X. Chen, C. Sun, H. Zhang, W. Pang, and J. Liu, "Enhanced sensitivity of mote2 chemical sensor through light illumination," Micromachines 8, 155 (2017).

[136] X. Luo, F. C. Chen, J. L. Zhang, Q. L. Pei, G. T. Lin, W. J. Lu, Y. Y. Han, C. Y. Xi, W. H. Song, and Y. P. Sun, " $T_{d^{-}}-\mathrm{MoTe}_{2}$ : A possible topological superconductor," Applied Physics Letters 109, 102601 (2016).

[137] B. E. Brown, "The crystal structures of $\mathrm{WTe}_{2}$ and high-temperature $\mathrm{MoTe}_{2}$," Acta Crystallographica 20, 268-274 (1966).

[138] X. Qian, J. Liu, L. Fu, and J. Li, "Quantum spin Hall effect in two-dimensional transition metal dichalcogenides," Science 346, 1344-1347 (2014).

[139] K. Deng, G. Wan, P. Deng, K. Zhang, S. Ding, E. Wang, M. Yan, H. Huang, H. Zhang, Z. Xu et al., "Experimental observation of topological Fermi arcs in type-II Weyl semimetal MoTe 2 ," Nature Physics 12, 1105-1110 (2016).

[140] A. N. Berger, E. Andrade, A. Kerelsky, D. Edelberg, J. Li, Z. Wang, L. Zhang, J. Kim, N. Zaki, J. Avila et al., "Temperature-driven topological transition in $1 T^{\prime}-\mathrm{MoTe}_{2}$," npj Quantum Materials 3, 1-8 (2018).

[141] Y. Qi, P. G. Naumov, M. N. Ali, C. R. Rajamathi, W. Schnelle, O. Barkalov, M. Hanfland, S.-C. Wu, C. Shekhar, Y. Sun et al., "Superconductivity in Weyl semimetal candidate $\mathrm{MoTe}_{2}$," Nature communications 7, 1-7 (2016). 
[142] S. Yang, C. Wang, H. Sahin, H. Chen, Y. Li, S.-S. Li, A. Suslu, F. M. Peeters, Q. Liu, J. Li, and S. Tongay, "Tuning the Optical, Magnetic, and Electrical Properties of $\mathrm{ReSe}_{2}$ by Nanoscale Strain Engineering," Nano Letters 15, 16601666 (2015).

[143] E. Torun, H. Sahin, S. Cahangirov, A. Rubio, and F. M. Peeters, "Anisotropic electronic, mechanical, and optical properties of monolayer $\mathrm{WTe}_{2}$," Journal of Applied Physics 119, 074307 (2016).

[144] L. Zhou, S. Huang, Y. Tatsumi, L. Wu, H. Guo, Y.-Q. Bie, K. Ueno, T. Yang, Y. Zhu, J. Kong, R. Saito, and M. Dresselhaus, "Sensitive Phonon-Based Probe for Structure Identification of $1 T^{\prime}-\mathrm{MoTe}_{2}$," Journal of the American Chemical Society 139, 8396 (2017).

[145] S. M. Oliver, R. Beams, S. Krylyuk, I. Kalish, A. K. Singh, A. Bruma, F. Tavazza, J. Joshi, I. R. Stone, S. J. Stranick, A. V. Davydov, and P. M. Vora, "The structural phases and vibrational properties of $\mathrm{Mo}_{1}-x \mathrm{~W}_{x} \mathrm{Te}_{2}$ alloys," 2D Materials 4, 045008 (2017).

[146] Y. Wang, J. Xiao, H. Zhu, Y. Li, Y. Alsaid, K. Y. Fong, Y. Zhou, S. Wang, W. Shi, Y. Wang, A. Zettl, E. J. Reed, and X. Zhang, "Structural phase transition in monolayer $\mathrm{MoTe}_{2}$ driven by electrostatic doping," Nature 550, 487 (2017).

[147] Y. Tan, F. Luo, M. Zhu, X. Xu, Y. Ye, B. Li, G. Wang, W. Luo, X. Zheng, N. Wu, Y. Yu, S. Qin, and X.-A. Zhang, "Controllable 2H-to-1T' phase transition in few-layer $\mathrm{MoTe}_{2}, "$ Nanoscale 10, 19964-19971 (2018).

[148] M. Vellinga, R. de Jonge, and C. Haas, "Semiconductor to metal transition in $\mathrm{MoTe}_{2}$," Journal of Solid State Chemistry 2, 299 - 302 (1970).

[149] C. Manolikas, J. van Landuyt, and S. Amelinckx, "Electron microscopy and electron diffraction study of the domain structures, the dislocation fine structure, and the phase transformations in $\beta-\mathrm{MoTe}_{2}, "$ physica status solidi (a) 53, 327-338 (1979).

[150] L. F. Mattheiss, "Band structures of transition-metal-dichalcogenide layer compounds," Phys. Rev. B 8, 3719-3740 (1973).

[151] H. P. Hughes and R. H. Friend, "Electrical resistivity anomaly in $\beta-\mathrm{MoTe}_{2}$ (metallic behaviour)," Journal of Physics C: Solid State Physics 11, L103 (1978).

[152] J. Jiang, Z. K. Liu, Y. Sun, H. F. Yang, C. R. Rajamathi, Y. P. Qi, L. X. Yang, C. Chen, H. Peng, C.-C. Hwang, S. Z. Sun, S.-K. Mo, I. Vobornik, J. Fujii, S. S. P. Parkin, C. Felser, B. H. Yan, and Y. L. Chen, "Signature of type-II Weyl semimetal phase in $\mathrm{MoTe}_{2}$," Nature Communications 8, 13973 (2017). 
[153] S. Manzeli, D. Ovchinnikov, D. Pasquier, O. V. Yazyev, and A. Kis, "2D transition metal dichalcogenides," Nature Reviews Materials 2, 17033 (2017).

[154] A. A. Soluyanov, D. Gresch, Z. Wang, Q. Wu, M. Troyer, X. Dai, and B. A. Bernevig, "Type-II Weyl Semimetals," Nature 527, 495 (2015).

[155] S. Barua, M. C. Hatnean, M. R. Lees, and G. Balakrishnan, "Signatures of the Kondo effect in $\mathrm{VSe}_{2}$," Scientific Reports 7, 10964 (2017).

[156] J. Van Baren, G. Ye, J.-A. Yan, Z. Ye, P. Rezaie, P. Yu, Z. Liu, R. He, and C. H. Lui, "Stacking-dependent interlayer phonons in $3 \mathrm{R}$ and $2 \mathrm{H}-\mathrm{MoS}_{2}, " 2 \mathrm{D}$ Materials 6, 025022 (2019).

[157] R. Clarke, E. Marseglia, and H. P. Hughes, "A low-temperature structural phase transition in $\beta-\mathrm{MoTe}_{2}, "$ Philosophical Magazine B 38, 121-126 (1978).

[158] C. Heikes, I.-L. Liu, T. Metz, C. Eckberg, P. Neves, Y. Wu, L. Hung, P. Piccoli, H. Cao, J. Leao et al., "Mechanical control of crystal symmetry and superconductivity in Weyl semimetal $\mathrm{MoTe}_{2}$," Physical Review Materials 2, 074202 (2018).

[159] W. G. Dawson and D. W. Bullett, "Electronic structure and crystallography of $\mathrm{MoTe}_{2}$ and $\mathrm{WTe}_{2}$," Journal of Physics C: Solid State Physics 20, 6159 (1987).

[160] D. Puotinen and R. Newnham, "The crystal structure of $\mathrm{MoTe}_{2}, "$ Acta Crystallographica 14, 691-692 (1961).

[161] J. A. Schneeloch, C. Duan, J. Yang, J. Liu, X. Wang, and D. Louca, "Emergence of topologically protected states in the $\mathrm{MoTe}_{2}$ Weyl semimetal with layerstacking order," Physical Review B 99, 161105 (2019). 


\title{
APPENDIX A: COMMONLY USED ACRONYMS
}

\author{
AFM - Anti FerroMagnetic \\ BZ - Brillouin zone \\ CVT - Chemical transpor vapour \\ DFT - Density functional theory \\ FM - Ferromagnetic \\ High- $\mathrm{T}_{c}-$ High temperature superconductor \\ HTSC - High temperature superconductor \\ MIT - Metal-to Insulator transition \\ PDF - Pair Distribution Function \\ LINAC - Linear Accelerator (linac) \\ LMDC - Layered Metal Chalcogenides \\ QPT - Quantum Phase Transition \\ SCES - Strongly Correlated Electronic Systems \\ SNS - Spallation Neutron Source \\ TMD - Transition Metal Dichalcogenide \\ TMO - Transition metal oxide \\ TOF - Time-of-Flight \\ 2D - Two-Dimensional \\ 2DM -Two-Dimensional Material \\ WSM - Weyl Semi Metal
}




\title{
CURRICULUM VITA
}

\author{
Alaa Alfailakawi
}

\section{Education}

M.S. , University of Massachusetts, Lowell, June 2015

M.S. , University of Iowa, Iowa City, June 2012

B.S. Kuwait University, Kuwait, June 1998

\section{Professional Societies}

American Physical Society (2018-present)

\section{Publications}

1. Site-selective electronic structure of pure and doped Ca2O3Fe3S2 L. Craco, B. Freelon, A. M. Alafailakawi, B. Karki, and S. Leoni published 20 July 2018 - PHYSICAL REVIEW B 98, 045130 (2018).

2.Local structural study of novel Mott-insulating cousins of the iron pnictides. Karki, B., Alfailakawi, A., Frandsen, B.A., Neuefeind, J.C., Everett, M. and Freelon, B. Crystallography, 75, p.a376. (2019)

\section{Posters}

1. 3rd Annual Modern Methods in Rietveld Refinement for Structural Analysis, school poster title: Exploring the Phase Diagram of Novel Iron Oxychalcogenides 2. GSA meeting march 2018, University of Louisville, Poster title : Structural and Magnetic Properties of a New Mott Insulator. 